$$
\begin{gathered}
\text { UNIVERSIDADE DE SÃO PAULO } \\
\text { ESCOLA DE ENGENHARIA DE SÃO CARLOS } \\
\text { DEPARTAMENTO DE ENGENHARIA ELÉTRICA E DE COMPUTAÇÃO } \\
\text { PROGRAMA DE PÓS-GRADUAÇÃO EM ENGENHARIA ELÉTRICA }
\end{gathered}
$$

RODRIGO BARRIVIERA

CONDICIONADOR UNIFICADO DE QUALIDADE DE ENERGIA UTILIZADO PARA COMPENSAÇÃO ATIVA DE POTÊNCIA EM REDES DE DISTRIBUIÇÃO 

RODRIGO BARRIVIERA

\section{CONDICIONADOR UNIFICADO DE QUALIDADE DE ENERGIA UTILIZADO PARA COMPENSAÇÃO ATIVA DE POTÊNCIA EM REDES DE DISTRIBUIÇÃO}

Tese apresentada ao Programa de Pós-Graduação em Engenharia Elétrica da Escola de Engenharia de São Carlos da Universidade de São Paulo, como parte dos requisitos para a obtenção do título de Doutor em Ciências.

Área de concentração: Sistemas Dinâmicos.

Orientador: Prof. Dr. Ricardo Quadros Machado. 


\section{AUTORIZO A REPRODUÇÃO TOTAL OU PARCIAL DESTE TRABALHO, POR QUALQUER MEIO CONVENCIONAL OU ELETRÔNICO, PARA FINS DE ESTUDO E PESQUISA, DESDE QUE CITADA A FONTE.}

Ficha catalográfica elaborada pela Biblioteca Prot. Dr. Sérgio Rodrigues Fontes da EESC/USP com os dados inseridos pelo(a) autor(a).

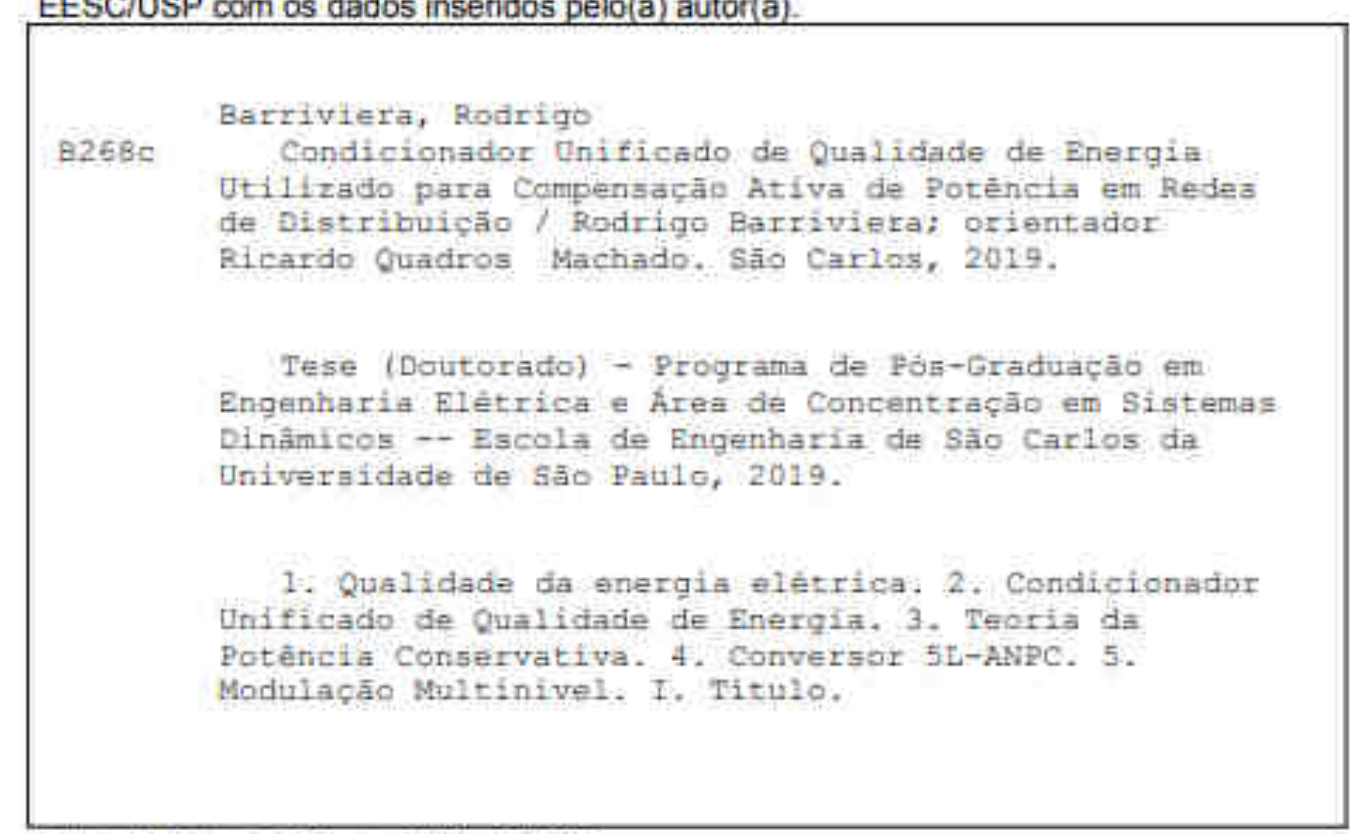

Eduardo Graziosi Silva - CRB - 8/8907 


\section{FOLHA DE JULGAMENTO}

Candidato: Engentretro RODRIGO BARRIVIERA.

Titulo da fese: "Condicionador unificado de qualidade de energia utilzade

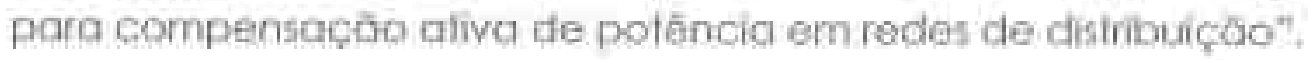

Data da defeser: $26 / 04 / 2019$

Comissāo Julaadora:

Resultado:

Prof. Associado Ricardo Quadros Mochodo.

APROVADC

(Orientador)

(Escola de Engenharia de Säo Carlas/Eesc)

Prof. Dr. Frank Gonzatti

(Universidade Federal de Santa Maria/UFSM)

AProungD

Prot. Dr. João Onafre Pereira Pinto

Almovano

(Unlversidade Federal de Santa Matia/UFSM)

Profa. Dra. Luciana Cambraia Leife

(Universidade Federal de Santa Maria/UFSM)

Anprovado

Prof. Dr. Manoel Luis de Aguiar

(Escolo de Engientiaria de Sâo Carios/EEsC)

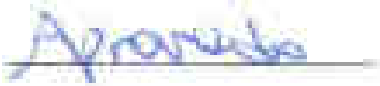

Coordenador do Programa de Pós-Graduaçà em Engenharia Elétrica:

Prot, Associado Ivan Nunes da Silva

Presidente da Comissóo de Pús-Graduoção:

Prot. Thular Murilo Araujo Romero 



\section{DEDICATÓRIA}

Dedico este trabalho a minha amada esposa Michele Caroline Delefrati Barriviera, ao meu amado filho querido Isaac Delefrati Barriviera, aos meus pais Romildo Barriviera e Celina Cardozo Marques Barriviera, aos meus sogros José Delefrati e Maria Izabel Constâncio Delefrati, e ao meu irmão Rodolfo Barriviera, sua esposa Caroline L. M. Barriviera, e meu sobrinho Miguel M. Barriviera, e também a minha irmã in memorial Rosemeri Barriviera. 



\section{AGRADECIMENTOS}

A Deus, pelo maior de todos os presentes, a Vida, na qual podemos trilhar caminhos de conhecimento e amadurecimento.

A Virgem Maria pelo auxílio, força, proteção e intercessão que sempre pude contar em toda jornada da vida.

A Michele Caroline Delefrati Barriviera, minha grande esposa amada, por todo apoio, muita paciência e amor, incentivo, companheirismo e amizade, por sempre estar ao meu lado me apoiando no decorrer de cada etapa da vida.

Ao meu filho amado, Isaac Delefrati Barriviera, por me proporcionar momentos únicos, alegres, fatores esses que me ajudaram a perseverar e nunca desistir.

A minha família, meus pais, meu irmão, cunhada e sobrinho, meus sogros por acreditarem em mim, que sempre me incentivaram e apoiaram.

Ao meu orientador, o Prof. Ricardo Quadros Machado, pelo apoio durante esta jornada, pelas sugestões técnicas e pessoais, pela orientação prestada durante o decorrer do curso, e na elaboração deste projeto.

A Profa. Vilma A. Oliveira, pela amizade e confiança.

Aos docentes da banca de avaliação, Prof. Dr. Frank Gonzatti, Prof. Dr. João Onofre Pereira Pinto, Profa. Dra. Luciana Cambraia Leite e Prof. Dr. Manuel Luis de Aguiar pelas contribuições deste trabalho.

A todos os docentes do Programa de Pós-Graduação em Engenharia Elétrica, do Departamento de Engenharia Elétrica e de Computação, da Escola de Engenharia de São Carlos - EESC, pela troca de conhecimentos que enriqueceram os resultados desta pesquisa.

Aos amigos e companheiros de trabalho no Instituto Federal do Paraná - IFPR pelo imenso apoio.

Aos amigos e parceiros de doutorado, Rodrigo Augusto Modesto, William César de Almeida Pereira, Leonardo Bruno Garcia Campanhol, Edson Junior Acordi e André Sanches Fonseca Sobrinho pelo companheirismo e troca de informações.

Aos amigos e parceiros do Laboratório de Fontes Alternativas e Processamento de Energia - LAFAPE, Magossi, Elian, Wagner, Daniel, Guilherme, Plinio, Augusto, Marina, Klebber e Matheus por toda cumplicidade, ideias compartilhadas e momentos de interação. 
A República Alabama, Rodolpho, Renzo, Romulo, Wagner, Thales e Jonas pelos momentos de companheirismo, acolhimento e distração.

Aos funcionários da SEL, pelo auxílio e atendimento nas tarefas que possibilitaram o andamento deste trabalho.

A Paróquia Nossa Senhora de Fátima - Diocesse de São Carlos, representada pelo Pároco Padre Jonas Rafael da Silva e toda comunidade, por toda acolhida e pelas palavras de sabedoria.

A Metalúrgica do Sr. Elias Brito, por ter cedido espaço para o desenvolvimento do protótipo.

Ao IFPR, por ter me concedido afastamento para o desenvolvimento deste trabalho.

A Fundação Araucária e Capes pela bolsa de estudos, CP 13/2014, 166/2015. 
קִִִ̣., Yitshakak. 
“ A minha meta está no alto, está no céu! Deixa passar o que passou, não olhe para trás. O céu é para quem sonha grande, pensa grande, ama grande e tem a coragem de viver pequeno. Isso é o céu! "

Padre Léo. 


\section{RESUMO}

BARRIVIERA, Rodrigo. Condicionador Unificado de Qualidade de Energia utilizado para Compensação Ativa de Potência em Redes de Distribuição. 2019.

174 f. Tese de Doutorado - Escola de Engenharia de São Carlos, Universidade de São Paulo, São Carlos, 2019.

A crescente demanda por fontes alternativas de energia, voltada principalmente a minimizar impactos ambientais provenientes do uso de combustíveis fósseis na geração de energia elétrica, tem impulsionado as pesquisas tanto no âmbito acadêmico como no setor privado. Dessa forma, estudos científicos vêm abordando painéis fotovoltaicos, aerogeradores, células combustível, dentre outros, no sentido de gerar energia limpa por meio de conversores estáticos CC-CC e CC-CA, conectados na rede de distribuição elétrica, configurando um cenário de Geração Distribuída. Devido ao uso de tais conversores, têm-se introduzido perturbações harmônicas nas redes de distribuição. Sendo assim, a qualidade de energia tornouse uma questão de suma importância no contexto energético contemporâneo, de tal modo que esta tese se apresenta um estudo voltado ao desenvolvimento e implementação de uma plataforma experimental de um Condicionador Unificado de Qualidade de Energia (UPQC) monofásico com compensação ativa de potência série e paralela, com uma potência de $1 \mathrm{~kW}$, capaz de minimizar perturbações harmônicas em redes de distribuição. O sistema realiza a supressão de harmônicos e a compensação de afundamentos e elevações de tensão em redes elétricas, além de manter a tensão na carga regulada e com baixa taxa de distorção harmônica. Além disso, esse dispositivo realiza compensação de potência reativa e supressão de harmônicos da corrente de carga, o que resulta em uma efetiva correção do fator de potência. Nesse sentido, o dispositivo atua como um filtro ativo universal, realizando compensação da corrente de entrada, como também da tensão de saída. Para este fim, dois conversores monofásicos ANPC de 5 níveis são empregados, sendo estes 0 filtro ativo série (FAS), que atua como fonte de corrente senoidal, e o filtro ativo paralelo (FAP), que atua como fonte de tensão senoidal, em que ambos são controlados de tal forma a operar em fase com a tensão da rede elétrica. Com o objetivo de realizar as compensações harmônicas, é utilizado o algoritmo de extração de referência, conhecido como Compensação Cooperativa baseada na Teoria de Potência Conservativa.

Palavras-chave: Qualidade da energia elétrica. Condicionador Unificado de Qualidade de Energia. Teoria da Potência Conservativa. Conversor 5L-ANPC. Modulação multinível. 



\begin{abstract}
BARRIVIERA, Rodrigo. Unified power quality conditioner used for active power compensation in distribution networks. 2019. 174 p. Ph.D. Thesis - São Carlos School of Engineering, University of São Paulo, 2019.

The growing demand for alternative sources of energy, aimed mainly at minimizing environmental impacts from the use of fossil fuels in electric power generation, has driven research in both the academic and private sectors. Thus, scientific studies have addressed photovoltaic panels, aerogenerators, fuel cells, among others, in order to generate clean energy by means of static converters CC-CC and CC-CA, connected in the electric distribution network, configuring the Distributed Generation. Due to the use of such converters, harmonic disturbances have been introduced in the distribution networks. Thus, the quality of energy has become a matter of paramount importance in the contemporary energy context, such that this thesis presents a study aimed at the development and implementation of an experimental platform of a single-phase Unified Energy Quality Conditioner (UPQC) with active series and parallel power compensation, with a power of $1 \mathrm{~kW}$, capable of minimizing harmonic disturbances in distribution networks. The system performs the suppression of harmonics and the compensation of sags and tensions in electrical networks, besides maintaining the voltage at the regulated load and with a low harmonic distortion rate. In addition, this device performs reactive power compensation and harmonic suppression of the load current, which results in an effective power factor correction. In this sense, the device acts as a universal active filter, performing compensation of the input current, as well as the output voltage. For this purpose, two 5-level ANPC single-phase converters are used, which are the series active filter (FAS), which acts as a sinusoidal current source, and the parallel active filter (FAP), which acts as a sinusoidal voltage source, in that both are controlled in such a way to operate in phase with the mains voltage. In order to perform the harmonic compensations, the reference extraction algorithm, known as Cooperative Compensation based on the Conservative Power Theory, is used.
\end{abstract}

Keywords: Power quality. Unified power quality conditioner. Conservative Power Theory. 5L-ANPC converter. Multilevel modulation. 



\section{LISTA DE FIGURAS}

Figura 2.1 - Diagrama em blocos simplificado de um sistema UPQC ........................40

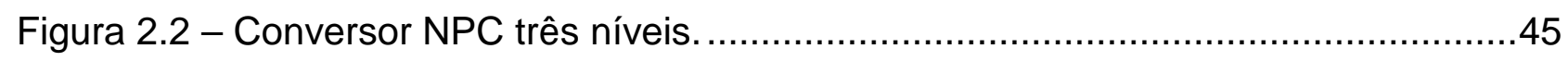

Figura 2.3 - Conversor Capacitor Flutuante - três níveis. ..................................47

Figura 2.4 - Inversor em Cascata (Castated Inverters) três níveis. ............................48

Figura 2.5 - Active Neutral Point Clamped (ANPC) - 3 níveis monofásico.....................50

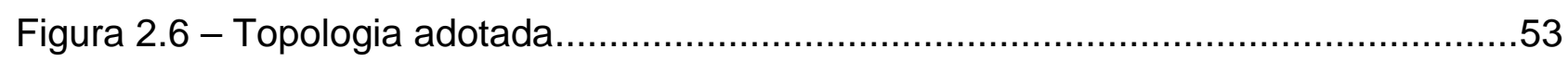

Figura 3.1 - Active Neutral Point Clamped (ANPC) - 5 níveis monofásico. .....................55

Figura 3.2 - Estado de chaveamento de cada nível. .......................................56

Figura 3.3 - Sinal de saída do conversor ANPC cinco níveis. ...................................59

Figura 3.4 - Dispositivos semicondutores e elementos passivos que são chaveados em

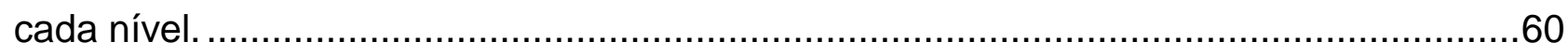

Figura 3.5 - Forma de onda da saída do inversor chaveado e do modelo matemático -

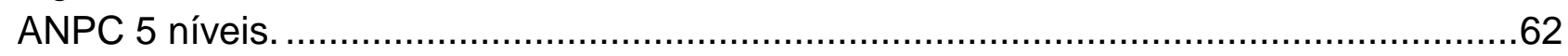

Figura 3.6 - Forma de onda da saída do inversor. (a) chaveado; (b) modelo teórico.......62

Figura 3.7 - (a) Phase Disposition (PH); (b) Padrão dos pulsos PWM. Parâmetros: $M=1$,

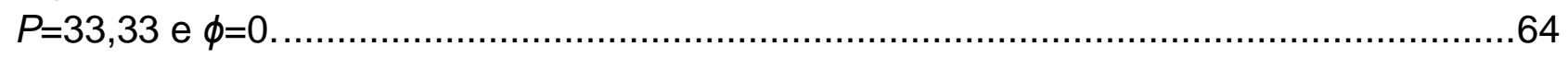

Figura 3.8 - Fluxograma da lógica de implementação da modulação multinível ANPC 5

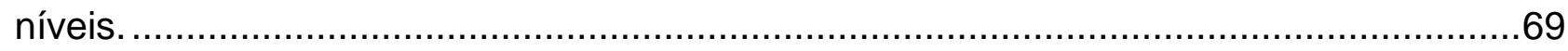

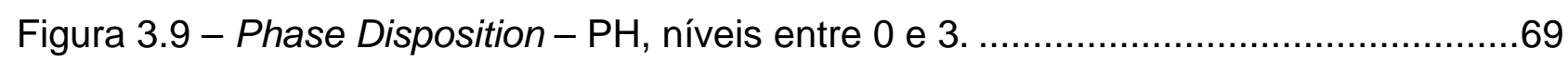

Figura 3.10 - Pulsos S1, S3 e S5 - Phase Disposition - PH.................................. 70

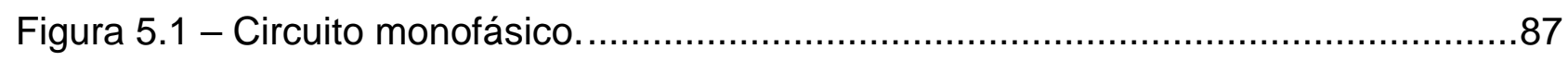

Figura 5.2 - Circuito monofásico do conversor série de corrente tipo ANPC cinco níveis conectado em série com a rede elétrica.........................................................89

Figura 5.3 - Circuito equivalente considerando as indutâncias de dispersão e resistências do transformador de acoplamento série......................................................90

Figura 5.4 - Circuito equivalente do conversor série. .........................................91

Figura 5.5 - Modelo do conversor série de potência...........................................91 
Figura 5.6 - Diagrama de blocos das malhas de controle de corrente do conversor série.

Figura 5.7 - Diagrama em blocos de laço aberto de controle de corrente do conversor série

Figura 5.8 - Circuito trifásico do conversor paralelo de tensão tipo ANPC cinco níveis ...95

Figura 5.9 - Circuito equivalente do conversor paralelo de tensão por fase. .96

Figura 5.10 - Diagrama de blocos das malhas de controle de tensão e corrente do conversor paralelo

Figura 5.11 - Diagrama de blocos da malha interna do controle de corrente do conversor paralelo de tensão.

Figura 5.12 - Diagrama de blocos da malha externa de tensão do conversor paralelo de tensão sem o controlador PI.

Figura 5.13 - Diagrama de blocos da malha externa de tensão do conversor paralelo de tensão com o controlador PI...

Figura 5.14 - Diagrama de bloco da malha de controle da tensão do elo CC 102

Figura 5.15 - Diagrama do circuito simplificado do desequilíbrio de tensão do elo CC ..103

Figura 5.16 - Diagrama de bloco da malha de controle do desequilíbrio de tensão do elo CC 104

Figura 5.17 - Diagrama de bloco do controlador Proporcional Ressonante (P-RES) .....107

Figura 5.18 - Resposta em frequência das funções $G_{P_{C S}+R E S_{k}}, G_{M A}$ S,$G_{c S}$ 109

Figura 5.19 - Resposta em frequência em laço fechado da malha de corrente com controlador P-RES

Figura 5.20 - Mapa de polos da função de transferência de malha fechada da malha de corrente com controlador P-RES.

Figura 5.21 - Mapa de polos e zeros da função de transferência de malha fechada da malha de corrente com controlador P-RES.

Figura 5.22 - Resposta em frequência das funções $G_{c p i}, G_{M A_{i}}, G_{M F_{i}}$ 113

Figura 5.23 - Resposta em frequência das funções $G_{P_{c p}+R E S_{k}}, G_{M A_{v}}, G_{M A_{c v}}$ 114

Figura 5.24 - Resposta em frequência em laço fechado da malha de tensão com controlador P-RES e $P$ 
Figura 5.25 - Mapa de polos e zeros da função de transferência em laço fechado da malha de tensão com controlador P-RES e P.

Figura 5.26 - Resposta em frequência em laço fechado da malha de tensão do elo CC com controlador PI

Figura 5.27 - Curvas das potências aparentes normalizadas do conversor série $\left|S_{S} / S_{L}\right|$.

Figura 5.28 - Curvas das potências aparentes normalizadas do conversor paralelo $\left|S_{p} / S_{L}\right|$

Figura 5.29 - Fluxo de potência: $V_{s}>V_{L}$ e $k_{c c}=0$.

Figura 5.30 - Fluxo de potência: $V_{s}<V_{L}$ e $k_{c c}=0$.

Figura 5.31 - Fluxo de potência: $V_{s}=V_{L}$ e $k_{c c}=0$.

Figura 5.32 - Fluxo de potência: $V_{s}=V_{L}$ e $k_{c c}=0.1$

Figura 5.33 - Diagrama de blocos das malhas de controle do conversor paralelo para análise de estabilidade sob variações de carga.

Figura 5.34 - Mapa de polos da FT em malha fechada sob análise de estabilidade do conversor paralelo $\left(1 \mu H \leq L_{e q} \leq 2000 \mu H\right)$ e $\left(R_{e q}=0.5 \Omega\right)$. 123

Figura 5.35 - Mapa de polos da FT em malha fechada sob análise de estabilidade do conversor paralelo $\left(0.1 \Omega \leq R_{e q} \leq 100 \Omega\right)$ e $\left(R_{e q}=10 \mu \mathrm{H}\right)$.

Figura 5.36 - Diagrama de blocos das malhas de controle do conversor série para análise de estabilidade sob variações na tensão da rede elétrica.

Figura 5.37 - Mapa de polos da FT em malha fechada sob análise de estabilidade do conversor série $\left(1 \mu H \leq L_{e q} \leq 2000 \mu H\right)$ e $\left(R_{e q}=0.5 \Omega\right)$. . . . .

Figura 5.38 - Mapa de polos da FT em malha fechada sob análise de estabilidade do conversor série $\left(0.1 \Omega \leq R_{e q} \leq 100 \Omega\right)$ e $\left(R_{e q}=10 \mu H\right)$. 126

Figura 6.1 - Diagrama de blocos do protótipo UPQC simulado. 128

Figura 6.2 - Inversor de tensão 5L-ANPC em malha aberta: (a) tensão do capacitor de fase $V_{C f, M}$, tensão no inversor $V_{i n v}$, tensão de saída $V_{o}$ e corrente no capacitor de fase $I_{C f}$; (b) tensão no inversor $V_{i n v}$, tensão de saída $V_{o}$ e corrente no capacitor de fase $I_{C f}$.....

Figura 6.3 - UPQC: (a) e (b) tensão de entrada $v_{S}$, tensão de saída $v_{L}$ e tensão sobre o transformador de acoplamento $v_{C}$, corrente de entrada $i_{S}$, corrente de compensação paralela $i_{\text {cfp }}$ e corrente de carga $i_{L}$; (a) carga - RL, (b) carga - RC. 
Figura 6.4 - UPQC: tensão de entrada $v_{S}$, tensão de saída $v_{L}$ e tensão sobre 0 transformador de acoplamento; $v_{C}$; (a) carga RL; (b) carga RC.

Figura 6.5 - UPQC (a) e (b) tensão de entrada $v_{S}$, tensão de saída $v_{L}$ e tensão sobre o transformador de acoplamento $v_{C}$, corrente de entrada is, corrente de compensação paralela $i_{c f p}$ e corrente de carga $i_{L}$; (a) carga - RL, (b) carga - RC. 132

Figura 6.6 - UPQC com conteúdo harmônico em $v_{s}$ : (a) tensão de entrada $v_{S}$, tensão de saída $v_{L}$ e tensão sobre o transformador de acoplamento, $v_{C}$.

Figura 6.7 - UPQC: (a) e (b) $v_{S}>v_{L}$ e $v_{S}<v_{L}$ : tensão de entrada $v_{S}$, tensão de saída $v_{L}$ e tensão sobre o transformador de acoplamento; $v_{C}$; corrente de entrada is e corrente de carga $i_{L}$; carga RL.

Figura 6.8 - UPQC: (a) e (b) $v_{S}>v_{L}$ e $v_{S}<v_{L}$ : tensão de entrada $v_{S}$, tensão de saída $v_{L}$ e tensão sobre o transformador de acoplamento; $v_{C}$; corrente de entrada is e corrente de carga $i_{L}$; carga $\mathrm{RC}$.

Figura 6.9 - UPQC: (a) e (b) $v_{S}>v_{L}$ e $v_{S}<v_{L}$ : tensão de entrada $v_{S}$ com DHT de 8,42\%, tensão de saída $v_{L}$ e tensão sobre o transformador de acoplamento; $v_{C}$; corrente de entrada is e corrente de carga $i_{L}$; carga RL.

Figura 6.10 - UPQC: (a) e (b) $v_{S}>v_{L}$ e $v_{S}<v_{L}$ : tensão de entrada $v_{S}$ com DHT de 8,42\%, tensão de saída $v_{L}$ e tensão sobre o transformador de acoplamento; $v_{C}$; corrente de entrada is e corrente de carga $i_{L}$; carga $R C$

Figura 6.11 - UPQC com degrau de carga: (a) e (b) tensão de entrada $v_{S}$, tensão de saída $V_{L}$, corrente de entrada $i_{S}$, corrente de compensação paralela $i_{c f p}$ e corrente de carga $i_{L} ;$ (a) carga RL; (b) carga RC.

Figura 6.12 - UPQC com degrau de carga: (a) e (b) tensão do elo total CC $V_{C C T}$, tensão do elo superior $V_{C C 1}$ e tensão do elo inferior $V_{C C 2}$, corrente de entrada is; (a) carga RL; (b) carga RC.

Figura 7.1 - Foto do protótipo UPQC (parte da frente).

Figura 7.2 - Foto do protótipo UPQC (parte de trás).

Figura 7.3 - Diagrama de blocos do protótipo UPQC implementado em laboratório.

Figura 7.4 - Inversor de tensão 5L-ANPC em malha aberta: (a) e (b) tensão do capacitor de fase $V_{C f, M}(100 \mathrm{~V} / \mathrm{div}, 5 \mathrm{~ms} / \mathrm{div})$, tensão no inversor $V_{\text {inv }}(100 \mathrm{~V} / \mathrm{div}, 5 \mathrm{~ms} / \mathrm{div})$, tensão de saída $V_{o}(100 \mathrm{~V} / \mathrm{div}, 5 \mathrm{~ms} / \mathrm{div})$ e corrente no capacitor de fase $I_{C f}(10 \mathrm{~A} / \mathrm{div}, 5 \mathrm{~ms} / \mathrm{div})$ e (c) tensão no inversor $V_{\text {inv }}(100 \mathrm{~V} / \mathrm{div}, 5 \mathrm{~ms} / \mathrm{div})$, tensão de saída $V_{o}(100 \mathrm{~V} / \mathrm{div}, 5 \mathrm{~ms} / \mathrm{div})$ e corrente no capacitor de fase $I_{C f}(10 \mathrm{~A} / \mathrm{div}, 5 \mathrm{~ms} / \mathrm{div})$. 144

Figura 7.5 - UPQC: (a) tensão de saída $v_{L}(100 \mathrm{~V} / \mathrm{div}, 10 \mathrm{~ms} / \mathrm{div})$, corrente de entrada is (5A/div, $10 \mathrm{~ms} / \mathrm{div})$, corrente de compensação paralela $i_{\text {cfp }}(5 \mathrm{~A} / \mathrm{div}, 10 \mathrm{~ms} / \mathrm{div})$ e corrente de carga - RL iL (5A/div, 10ms/div); (b) tensão de saída $v_{L}(100 \mathrm{~V} / \mathrm{div}, 5 \mathrm{~ms} / \mathrm{div})$, corrente de 
entrada is (5A/div, $5 \mathrm{~ms} /$ div $)$, corrente de compensação paralela $i_{c f p}(5 \mathrm{~A} / \mathrm{div}, 5 \mathrm{~ms} / \mathrm{div})$ e corrente de carga - RC iL (5A/div, $5 \mathrm{~ms} /$ div).

Figura 7.6 - UPQC: (a) tensão de entrada $v_{S}(50 \mathrm{~V} / \mathrm{div}, 10 \mathrm{~ms} / \mathrm{div})$, corrente de entrada is (5A/div, $10 \mathrm{~ms} / \mathrm{div})$, tensão de saída $v_{L}(50 \mathrm{~V} / \mathrm{div}, 10 \mathrm{~ms} / \mathrm{div})$, corrente de carga $-\mathrm{RL} i_{L}(5 \mathrm{~A} / \mathrm{div}$, $10 \mathrm{~ms} / \mathrm{div})$; (b) tensão de entrada $v_{S}(50 \mathrm{~V} / \mathrm{div}, 10 \mathrm{~ms} / \mathrm{div})$, corrente de entrada is $(5 \mathrm{~A} / \mathrm{div}$, $10 \mathrm{~ms} / \mathrm{div})$, tensão de saída $v_{L}(50 \mathrm{~V} / \mathrm{div}, 10 \mathrm{~ms} / \mathrm{div})$, corrente de carga $-\mathrm{RC} i_{L}(5 \mathrm{~A} / \mathrm{div}$, $10 \mathrm{~ms} / \mathrm{div})$. 146

Figura 7.7 - UPQC com conteúdo harmônico em $v_{s}$ : (a) tensão de entrada $v_{S}(50 \mathrm{~V} / \mathrm{div}$, $10 \mathrm{~ms} / \mathrm{div})$, corrente de entrada is $(5 \mathrm{~A} / \mathrm{div}, 10 \mathrm{~ms} / \mathrm{div})$, tensão de saída $v_{L}(50 \mathrm{~V} / \mathrm{div}, 10 \mathrm{~ms} / \mathrm{div})$ e corrente de carga - RL iL (5A/div, $10 \mathrm{~ms} / \mathrm{div})$; (b) tensão de entrada $v_{S}(50 \mathrm{~V} / \mathrm{div}, 10 \mathrm{~ms} / \mathrm{div})$, corrente de entrada is (10A/div, $10 \mathrm{~ms} /$ div), tensão de saída $v_{L}(50 \mathrm{~V} / \mathrm{div}, 10 \mathrm{~ms} / \mathrm{div})$ e corrente de carga - RC $i_{L}(10 \mathrm{~A} / \mathrm{div}, 10 \mathrm{~ms} / \mathrm{div})$.

Figura 7.8 - UPQC: tensão de entrada $v_{S}(30 \mathrm{~V} / \mathrm{div}, 10 \mathrm{~ms} / \mathrm{div})$, tensão de saída $v_{L}(30 \mathrm{~V} / \mathrm{div}$, $10 \mathrm{~ms} / \mathrm{div})$ e tensão sobre o transformador de acoplamento; $v_{C}(5 \mathrm{~V} / \mathrm{div}, 10 \mathrm{~ms} / \mathrm{div}) ;$ carga RL.

Figura 7.9 - UPQC com conteúdo harmônico em vs: (a) tensão de entrada $v_{S}(30 \mathrm{~V} / \mathrm{div}$, $10 \mathrm{~ms} / \mathrm{div})$, tensão de saída $v_{L}(30 \mathrm{~V} / \mathrm{div}, 10 \mathrm{~ms} / \mathrm{div})$ e tensão sobre o transformador de acoplamento; $v_{C}(20 \mathrm{~V} / \mathrm{div}, 10 \mathrm{~ms} / \mathrm{div})$

Figura 7.10 - UPQC: (a) $\left(v_{S}>v_{L}\right)$ : tensão de entrada $v_{S}(30 \mathrm{~V} / \mathrm{div}, 10 \mathrm{~ms} / \mathrm{div})$, tensão de saída $v_{L}(30 \mathrm{~V} / \mathrm{div}, 10 \mathrm{~ms} / \mathrm{div})$ e tensão sobre o transformador de acoplamento; $v_{C}$ (30V/div, $10 \mathrm{~ms} / \mathrm{div})$; (b) $\left(v_{S}<v_{L}\right)$ : tensão de entrada $v_{S}(30 \mathrm{~V} / \mathrm{div}, 10 \mathrm{~ms} / \mathrm{div})$, tensão de saída $v_{L}$ $(30 \mathrm{~V} / \mathrm{div}, 10 \mathrm{~ms} / \mathrm{div})$ e tensão sobre o transformador de acoplamento; $v_{C}(30 \mathrm{~V} / \mathrm{div}$, $10 \mathrm{~ms} / \mathrm{div})$ 148

Figura 7.11 - UPQC: (a) $\left(v_{S}>v_{L}\right)$ : tensão de entrada $v_{S}(30 \mathrm{~V} / \mathrm{div}, 10 \mathrm{~ms} / \mathrm{div})$, tensão de saída $v_{L}(30 \mathrm{~V} / \mathrm{div}, 10 \mathrm{~ms} / \mathrm{div})$, corrente de entrada is $(5 \mathrm{~A} / \mathrm{div}, 10 \mathrm{~ms} / \mathrm{div})$ e corrente de carga - RL $i_{L}(5 \mathrm{~A} / \mathrm{div}, 10 \mathrm{~ms} / \mathrm{div}) ;$ (b) $\left(v_{S}<v_{L}\right)$ : tensão de entrada $v_{S}(30 \mathrm{~V} / \mathrm{div}, 10 \mathrm{~ms} / \mathrm{div})$, tensão de saída $v_{L}(30 \mathrm{~V} / \mathrm{div}, 10 \mathrm{~ms} / \mathrm{div})$, corrente de entrada is $(5 \mathrm{~A} / \mathrm{div}, 10 \mathrm{~ms} / \mathrm{div})$ e corrente de carga - RL iL (5A/div, 10ms/div). 148

Figura 7.12 - UPQC com conteúdo harmônico em $v_{S}$ : (a) $\left(v_{S}>v_{L}\right)$ : tensão de entrada $v_{S}$ (30V/div, $10 \mathrm{~ms} / \mathrm{div})$, tensão de saída $V_{L}(30 \mathrm{~V} / \mathrm{div}, 10 \mathrm{~ms} / \mathrm{div})$ e tensão sobre o transformador de acoplamento; $v_{C}(30 \mathrm{~V} / \mathrm{div}, 10 \mathrm{~ms} / \mathrm{div})$; (b) $\left(v_{S}<v_{L}\right)$ : tensão de entrada $v_{S}(30 \mathrm{~V} / \mathrm{div}$, $10 \mathrm{~ms} / \mathrm{div})$, tensão de saída $V_{L}(30 \mathrm{~V} / \mathrm{div}, 10 \mathrm{~ms} / \mathrm{div})$ e tensão sobre o transformador de acoplamento $v_{C}(30 \mathrm{~V} / \mathrm{div}, 10 \mathrm{~ms} / \mathrm{div})$. 149

Figura 7.13 - UPQC com conteúdo harmônico em $v_{S}$ : (a) $\left(v_{S}>v_{L}\right)$ : tensão de entrada $v_{S}$ (30V/div, $10 \mathrm{~ms} / \mathrm{div})$, tensão de saída $v_{L}(30 \mathrm{~V} / \mathrm{div}, 10 \mathrm{~ms} / \mathrm{div})$, corrente de entrada is $(5 \mathrm{~A} / \mathrm{div}$, $10 \mathrm{~ms} / \mathrm{div})$ e corrente de carga $-\mathrm{RL} i_{L}(5 \mathrm{~A} / \mathrm{div}, 10 \mathrm{~ms} / \mathrm{div})$; (b) $\left(v_{S}<v_{L}\right)$ : tensão de entrada $v_{S}$ (30V/div, $10 \mathrm{~ms} / \mathrm{div})$, tensão de saída $v_{L}(30 \mathrm{~V} / \mathrm{div}, 10 \mathrm{~ms} / \mathrm{div})$, corrente de entrada is $(5 \mathrm{~A} / \mathrm{div}$, $10 \mathrm{~ms} / \mathrm{div})$ e corrente de carga $-\mathrm{RL} i_{L}(5 \mathrm{~A} / \mathrm{div}, 10 \mathrm{~ms} / \mathrm{div})$ 150 
Figura 7.14 - UPQC com degrau de carga: (a) tensão do capacitor de fase $V_{C f}(10 \mathrm{~V} / \mathrm{div}$, $200 \mathrm{~ms} / \mathrm{div})$, corrente de entrada is (50A/div, 200ms/div), corrente de compensação paralela $i_{\text {cfp }}(50 \mathrm{~A} / \mathrm{div}, 200 \mathrm{~ms} / \mathrm{div})$ e corrente de carga $-\mathrm{RL} i_{L}(50 \mathrm{~A} / \mathrm{div}, 200 \mathrm{~ms} / \mathrm{div}) ;(\mathrm{b})$ tensão do capacitor de fase $V_{C f, M}(50 \mathrm{~V} / \mathrm{div}, 200 \mathrm{~ms} / \mathrm{div})$, corrente de entrada is $(20 \mathrm{~A} / \mathrm{div}, 200 \mathrm{~ms} / \mathrm{div})$, corrente de compensação paralela $i_{c f p}(20 \mathrm{~A} / \mathrm{div}, 200 \mathrm{~ms} / \mathrm{div})$ e corrente de carga $-\mathrm{RC} i_{L}$ (20A/div, 200ms/div). 151

Figura 7.15 - UPQC com degrau de carga: (a) tensão de entrada $v_{S}(100 \mathrm{~V} / \mathrm{div}, 200 \mathrm{~ms} / \mathrm{div})$, corrente de entrada is (50A/div, $200 \mathrm{~ms} / \mathrm{div})$, corrente de compensação paralela $i_{c f p}(50 \mathrm{~A} / \mathrm{div}$, $200 \mathrm{~ms} / \mathrm{div})$ e corrente de carga - RL $i_{L}(50 \mathrm{~A} / \mathrm{div}, 200 \mathrm{~ms} / \mathrm{div})$; (b) tensão de entrada $v_{S}$ $(100 \mathrm{~V} / \mathrm{div}, 200 \mathrm{~ms} / \mathrm{div})$, corrente de entrada is $(20 \mathrm{~A} / \mathrm{div}, 200 \mathrm{~ms} / \mathrm{div})$, corrente de compensação paralela $i_{c f p}(20 \mathrm{~A} / \mathrm{div}, 200 \mathrm{~ms} / \mathrm{div})$ e corrente de carga $-\mathrm{RC} i_{L}(20 \mathrm{~A} / \mathrm{div}$, $200 \mathrm{~ms} / \mathrm{div})$. 151

Figura 7.16 - UPQC com degrau de carga: (a) tensão de saída $v_{L}(100 \mathrm{~V} / \mathrm{div}, 200 \mathrm{~ms} / \mathrm{div})$, corrente de entrada is (50A/div, $200 \mathrm{~ms} / \mathrm{div})$, corrente de compensação paralela $i_{c f p}(50 \mathrm{~A} / \mathrm{div}$, $200 \mathrm{~ms} / \mathrm{div})$ e corrente de carga - RL i $(50 \mathrm{~A} / \mathrm{div}, 200 \mathrm{~ms} / \mathrm{div})$; (b) tensão de saída $v_{L}$ $(100 \mathrm{~V} / \mathrm{div}, 200 \mathrm{~ms} / \mathrm{div})$, corrente de entrada is $(20 \mathrm{~A} / \mathrm{div}, 200 \mathrm{~ms} / \mathrm{div})$, corrente de compensação paralela $i_{c f p}(20 \mathrm{~A} / \mathrm{div}, 200 \mathrm{~ms} / \mathrm{div})$ e corrente de carga $-\mathrm{RC} i_{L}$ (20A/div, $200 \mathrm{~ms} / \mathrm{div})$ 152

Figura 7.17 - UPQC com degrau de carga - RC: (a) aumento de carga: tensão do elo total CC $V_{C C T}\left(50 \mathrm{~V} /\right.$ div, $500 \mathrm{~ms} /$ div), tensão do elo superior $\mathrm{V}_{C C 1}(50 \mathrm{~V} / \mathrm{div}, 500 \mathrm{~ms} / \mathrm{div})$ e tensão do elo inferior $\mathrm{V}_{C C 2}(50 \mathrm{~V} / \mathrm{div}, 500 \mathrm{~ms} / \mathrm{div})$; (b) retirada de carga: tensão do elo total $\mathrm{CC} \mathrm{V}_{C C T}$ $(50 \mathrm{~V} / \mathrm{div}, 500 \mathrm{~ms} / \mathrm{div})$, tensão do elo superior $\mathrm{V}_{c c 1}(50 \mathrm{~V} / \mathrm{div}, 500 \mathrm{~ms} / \mathrm{div})$ e tensão do elo inferior $\mathrm{V}_{C C 2}(50 \mathrm{~V} / \mathrm{div}, 500 \mathrm{~ms} / \mathrm{div})$. 152

Figura 7.18 - UPQC com degrau de carga - RC: tensão do elo total CC $\mathrm{V}_{C C T}$ (50V/div, $1.4 \mathrm{~s} / \mathrm{div})$, tensão do elo superior $\mathrm{V}_{C C 1}(50 \mathrm{~V} / \mathrm{div}, 1.4 \mathrm{~s} / \mathrm{div})$ e tensão do elo inferior $\mathrm{V}_{C C 2}$ (50V/div, $1.4 \mathrm{~s} / \mathrm{div})$. 153

Figura 7.19 - Espectros harmônicos - carga RL. 153

Figura 7.20 - Espectros harmônicos com tensão de entrada distorcida e carga RC......154 


\section{LISTA DE TABELAS}

Tabela 2.1 - Sequência de chaveamento - ANPC - três níveis. 51

Tabela 2.2 - Sequência de comutação ANPC três níveis. .51

Tabela 3.1 - Estado das chaves semicondutoras e o impacto do capacitor de fase (flutuante) - ANPC cinco níveis (Barbosa et al., 2005). .56

Tabela 3.2 - Seleção dos estados de chaveamento - ANPC cinco níveis. 59

Tabela 5.1 - Especificações para projeto do controlador P-RES de corrente do conversor série. 108

Tabela 5.2 - Especificações para projeto do controlador P de corrente e P-RES de tensão do conversor paralelo.

Tabela 5.3 - Especificações para projeto do controlador PI de tensão do elo CC. 115

Tabela 6.1 - Parâmetros de simulação 129

Tabela 7.1 - Parâmetros utilizados no experimento implementado. 143 


\section{LISTA DE ABREVIATURAS E SIGLAS}

ANEEL Agência Nacional de Energia Elétrica;

ANPC Active Neutral Point Clamped;

APF Active Power Filters;

CC Corrente Contínua;

CHB Cascated H-Bridge;

CO2 Dióxidos de carbono;

CPT Conservative Power Theory (Teoria da Potência Conservativa);

DCC Diode Clamped Converter;

DHT Distorção harmônica total;

DSP Digital Signal Processor;

EIA Administração de Informação de Energia dos EUA;

EMI Electromagnetic Interference;

EPRI Instituto de Pesquisas de Energia Elétrica dos EUA;

EUA Estados Unidos da América;

FAP Filtros Ativos de Potência;

FAPP Filtro Ativo de Potência Paralelo;

FAPS Filtro Ativo de Potência Série;

FC Flying Capacitor;

GD Geração Distribuída;

IEC International Electrotechnical Commission;

IEEE Institute of Electrical and Electronics Enginners;

IGBT Insulated Gate Bipolar Transistor;

IUPQC Unified Power Quality Conditioner with Inverted Topology;

LS-PWM Level Shifted Pulse Width Modulation;

MCU Microcontrolador de ponto flutuante;

NPC Neutral Point Clamped;

PAC Ponto de acoplamento comum;

PH-PWM Phase Disposition Pulse Width Modulation;

PLL Phase-Locked Loop;

PRODIST Procedimentos de Distribuição de Energia Elétrica no Sistema Elétrico Nacional;

PROINFA Programa de Incentivo às Fontes Alternativas de Energia Elétrica; 
PS-PWM Phase Shifted Pulse Width Modulation;

PWM Senoidal Pulse Width Modulation;

QEE Qualidade de Energia Elétrica;

RMS Root Mean Square;

SHE Eliminação seletiva de harmônicos;

SVM Modulação space vector;

UPQC Unified Power Quality Conditioner (Condicionador Unificado de Qualidade de Energia);

UPS Uninterruptible Power Supply;

VTCD Variações de tensão de curta duração. 


\section{LISTA DE SÍMBOLOS}

Símbolo

$C_{f}$

$C_{f p}$

$i_{L h}$

$v_{s h}$

$i_{\text {as }}$

$i_{a}$

$i_{L}$

$i_{C f}$

$i_{g}$

$i_{r s}$

$i_{r}$

$i_{v}$

$W_{r}$

Wr

w

$\lambda_{U}$

$k_{c c}$

$\cos (\varphi)$

$\lambda_{D}$

$\lambda$

$\lambda_{Q}$

$f_{s a}$

$f_{s}$

$G_{c}$
Descrição

Capacitância do capacitor de fase (flutuante)

Capacitor de filtragem do conversor paralelo

Componente harmônica da corrente da carga

Componentes harmônicas da tensão da rede elétrica

Corrente ativa dispersa, propriedade conservativa

Corrente ativa, propriedade conservativa

Corrente da carga

Corrente de saída do capacitor de fase (flutuante)

Corrente harmônica gerada pela carga

Corrente reativa dispersa

Corrente reativa, propriedade conservativa

Corrente residual, propriedade conservativa

Energia Reativa

Energia reativa coletiva, propriedade conservativa

Energia reativa instantânea, propriedade conservativa

Fator de assimetria

Fator de carga do elo CC

Fator de deslocamento

Fator de não-linearidade

Fator de potência

Fator de reatividade

Frequência de amostragem do conversor $A / D$

$\mathrm{Hz}$

$\mathrm{Hz}$
Unidade

F

F

A

V

A

A

A

A

A

A

A

A

J

J

J

Frequência de chaveamento

Função de transferência do controlador proporcional integral (PI) 


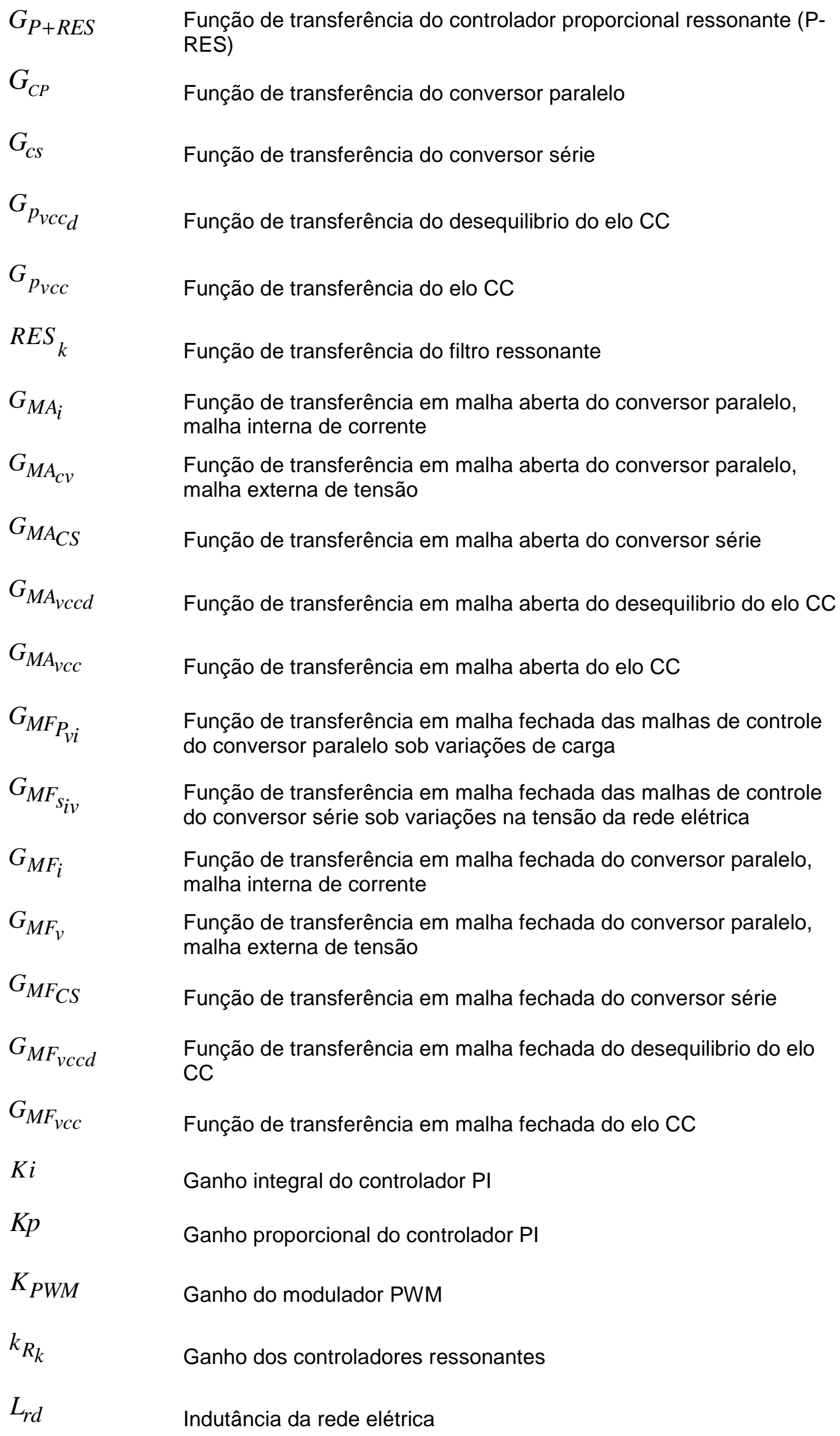

Função de transferência do controlador proporcional ressonante (PFunção de transferência do conversor paralelo

Função de transferência do conversor série

Função de transferência do desequilibrio do elo CC

Função de transferência do elo CC

Função de transferência do filtro ressonante

Função de transferência em malha aberta do conversor paralelo, a co interna de corrente

Função de transferência em malha aberta do conversor paralelo, Função de transferência em malha aberta do conversor série

Função de transferência em malha aberta do desequilibrio do elo CC

Função de transferência em malha aberta do elo CC

Função de transferência em malha fechada das malhas de controle

Função de transferência em malha fechada das malhas de controle Função de transferência em malha fechada do conversor paralelo, malha interna de corrente

Função de transferência em malha fechada do conversor paralelo, malha externa de tensão

Função de transferência em malha fechada do desequilibrio do elo

Função de transferência em malha fechada do elo CC

Ganho integral do controlador PI

Ganho proporcional do controlador PI

Ganho do modulador PWM

Ganho dos controladores ressonantes

Indutância da rede elétrica 


\begin{tabular}{|c|c|c|}
\hline$L_{f p}$ & Indutância de acoplamento do conversor paralelo & $\mathrm{H}$ \\
\hline$L_{f s}$ & Indutância de acoplamento do conversor série & $\mathrm{H}$ \\
\hline$L_{d p}$ & Indutância de dispersão do primário do transformador & $\mathrm{H}$ \\
\hline$L_{d \mathrm{~s}}$ & Indutância de dispersão do secundário do transformador & $\mathrm{H}$ \\
\hline$L_{e q_{s}}$ & Indutância equivalente do conversor série & $\mathrm{H}$ \\
\hline$\hat{x}(t)$ & Integral imparcial de $x$ & \\
\hline$R$ & Modelo matemático do conversor multinível ANPC 5 níveis & \\
\hline$\|\underline{x}\|$ & Norma do vetor $x$ & \\
\hline$n$ & Número de níveis do conversor multinível & \\
\hline$i_{L f}$ & Parcela fundamental da corrente da carga & A \\
\hline$v_{s f}$ & Parcela fundamental da tensão da rede elétrica & V \\
\hline$T$ & Período & s \\
\hline$S_{L}$ & Potência aparente da carga & VA \\
\hline$S_{p}$ & Potência aparente do conversor paralelo & VA \\
\hline$S_{S}$ & Potência aparente do conversor série & VA \\
\hline$P$ & Potência ativa coletiva, propriedade conservativa & W \\
\hline$P_{c c}$ & Potência ativa do elo CC & \\
\hline$p$ & Potência instantânea, propriedade conservativa & W \\
\hline Q & Potência Reativa & VA \\
\hline$D$ & Potência Residual & VA \\
\hline$R_{r d}$ & Resistência da rede elétrica & $\Omega$ \\
\hline$R_{d p}$ & Resistência do primário do transformador & $\Omega$ \\
\hline$R_{d s}$ & Resistência do secundário do transformador & $\Omega$ \\
\hline$R_{e q_{s}}$ & Resistência equivalente do conversor série & $\Omega$ \\
\hline$R_{L f p}$ & $\begin{array}{l}\text { Resistência equivalente do indutor de acoplamento do conversor } \\
\text { paralelo }\end{array}$ & \\
\hline$R_{L f s}$ & $\begin{array}{l}\text { Resistência equivalente do indutor de acoplamento do conversor } \\
\text { série }\end{array}$ & $\Omega$ \\
\hline
\end{tabular}




\begin{tabular}{|c|c|}
\hline$a b c$ & Sistema de coordenadas estacionárias abc \\
\hline$i_{L}\left(T D H_{i_{L}}\right)$ & TDH da corrente de carga \\
\hline$v_{L}$ & Tensão da carga \\
\hline$v_{s}$ & Tensão da rede elétrica \\
\hline$V_{\text {ref }\{R, S, T\}}$ & Tensão de referência \\
\hline $\mathrm{U}$ & Tensão do elo CC do conversor multínivel \\
\hline$V_{C C_{2}}$ & Tensão no capacitor inferior do elo CC \\
\hline$V_{C C_{1}}$ & Tensão no capacitor superior do elo CC \\
\hline$V_{C C_{T}}$ & Tensão no elo CC \\
\hline$V_{C f}$ & Tensão sobre o capacitor de fase (flutuante) \\
\hline$v_{c}$ & Tensão sobre o transformador de acoplamento série \\
\hline $\bar{x}$ & Valor médio da grandeza $x$ \\
\hline$\underline{\bar{x}}$ & Valor médio do vetor $x$ \\
\hline
\end{tabular}




\section{SUMÁRIO}

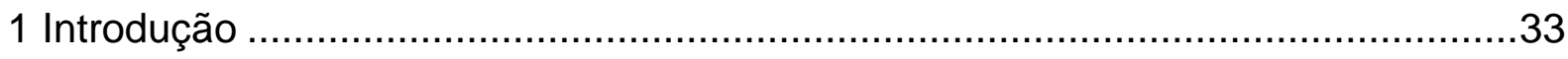

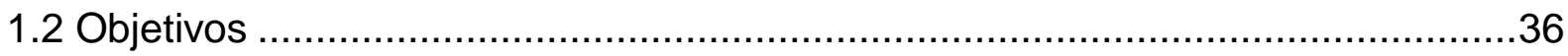

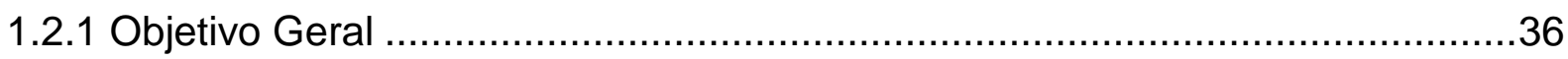

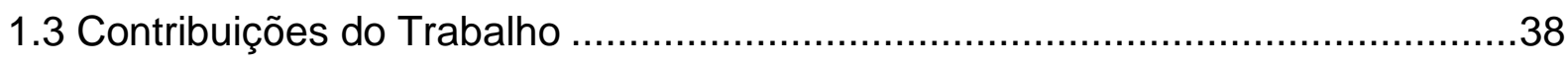

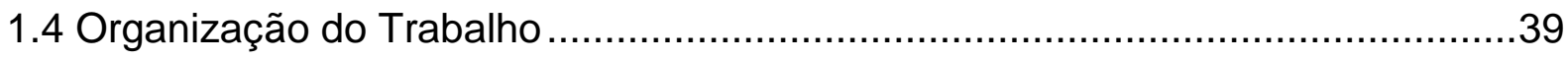

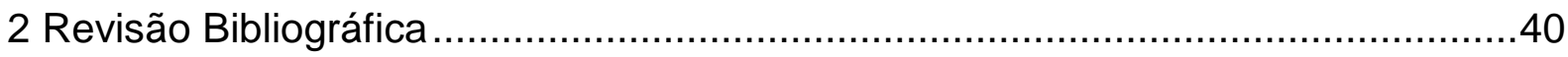

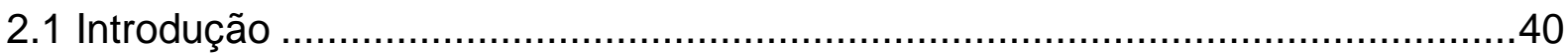

2.2 Condicionador de Qualidade de Energia Unificado (UPQC) …........................40

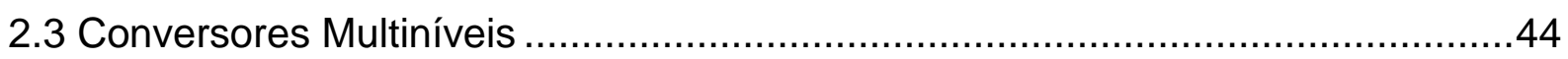

2.3.1 Ponto Neutro Grampeado a Diodo (Neutral Point Clamped - NPC) .................45

2.3.2 Capacitor Flutuante (Flying Capacitor - FC) ……....................................46

2.3.3 Inversores em Cascata com separação de Fontes de Corrente Contínua

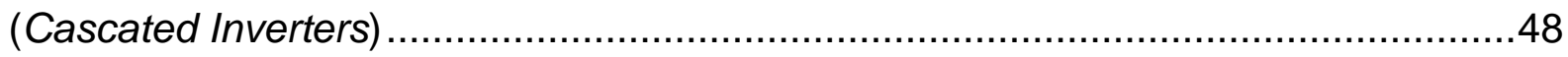

2.3.4 Inversor com Grampeamento Ativo do Neutro (Active Neutral Point Clamped -

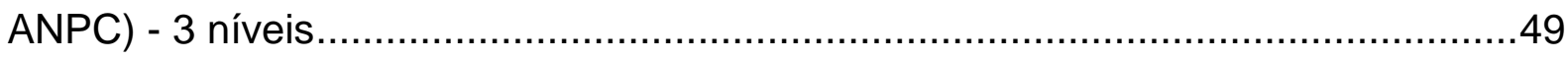

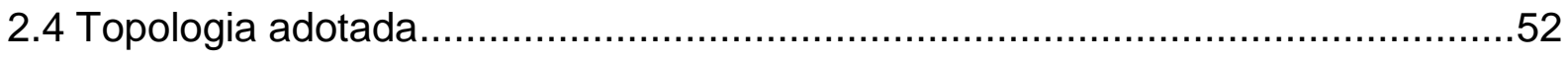

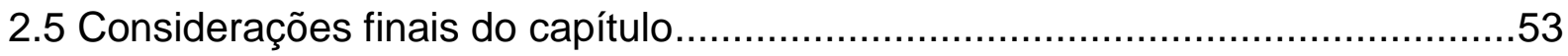

3 Inversor com Grampeamento Ativo do Neutro (Active Neutral Point Clamped -

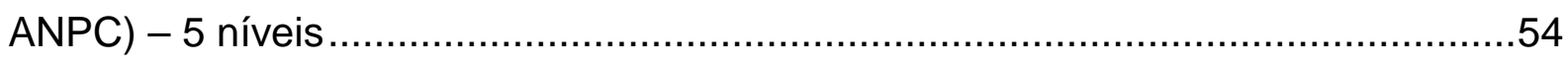

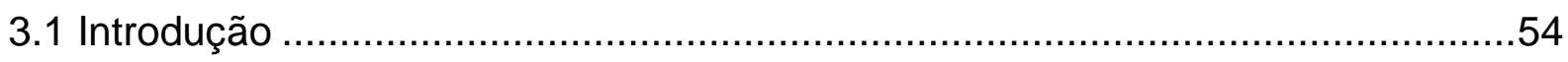

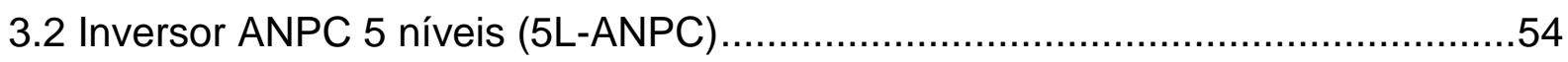

3.3 Modelo matemático do conversor multinível ANPC 5 níveis ..............................60

3.4 Modulação do conversor multinível ANPC 5 níveis ............................................63

3.5 Considerações finais do capítulo.....................................................................

4 Compensação Cooperativa baseada na Teoria da Potência Conservativa ............71

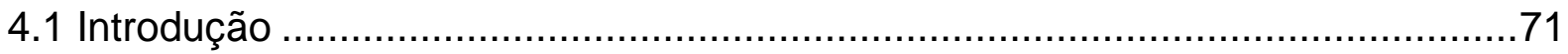

4.2 Potência instantânea e energia reativa instantânea...........................................71 


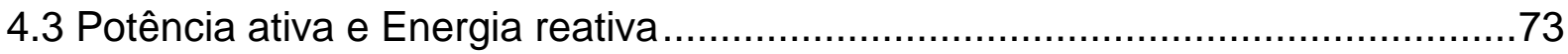

4.4 Definição de termos de corrente e potência em circuitos monofásicos ................74

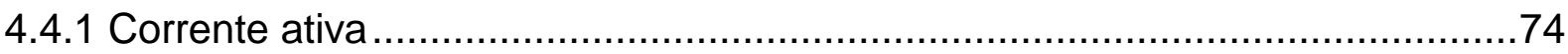

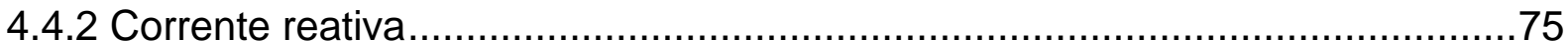

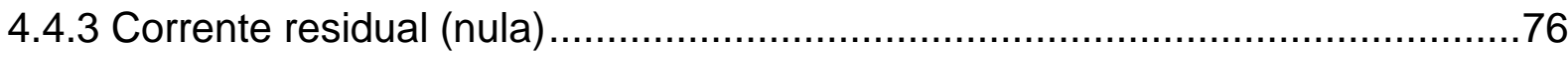

4.5 Decomposição completa da corrente em circuitos monofásicos .........................80

4.6 Potência aparente em circuitos monofásicos …………...................................

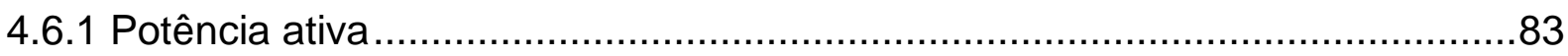

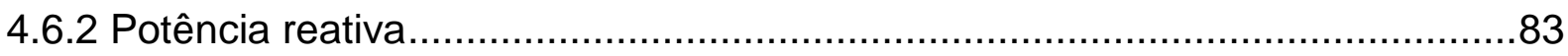

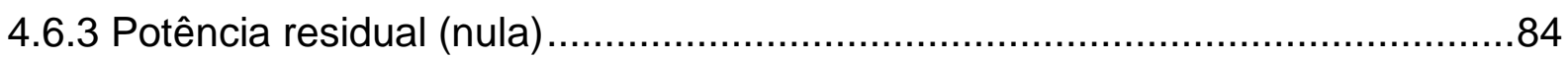

4.7 Fator de potência em circuitos monofásicos ……….......................................85

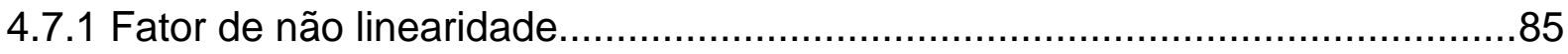

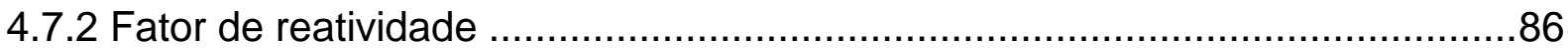

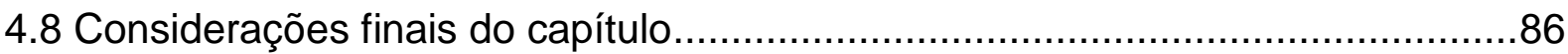

5 Descrição dos Modelos Matemáticos e Projeto dos Controladores .......................87

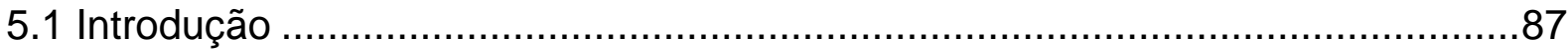

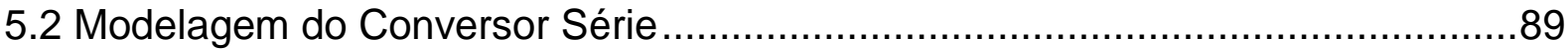

5.3 Modelagem da Malha de Controle de Corrente do conversor série ....................93

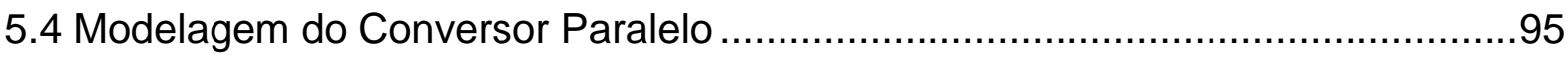

5.5 Modelagem da Malha de Controle de Tensão do conversor paralelo ..................97

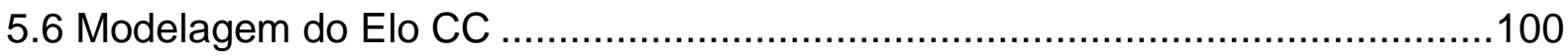

5.7 Modelagem da Malha de Controle do Elo CC ….........................................102

5.8 Modelagem do Desequilíbrio do Elo CC...................................................103

4.9 Modelagem da Malha de Controle do Desequilíbrio de Tensão do Elo CC ......104

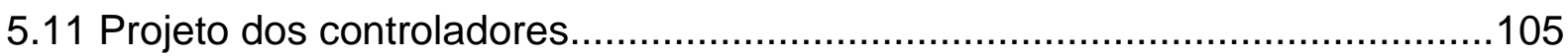

5.11.1 Projeto do controlador proporcional integral (PI) …...............................105

5.11.2 Projeto do controlador proporcional ressonante (P-RES) ...........................106 
5.11.3 Projeto do controlador P-RES da malha de controle do conversor série .....108

5.11.4 Projeto do controlador P-RES e P da malha de controle do conversor paralelo

5.11.5 Projeto do controlador PI da malha de controle do elo CC. 115

5.12 Fluxo de Potência do UPQC

5.13 Análise de estabilidade do UPQC

5.14 Considerações finais do capítulo 126

6 Simulações Computacionais 128

6.1 Introdução 128

6.2 Resultados de Simulações 130

6.3 Considerações finais do capítulo. 138

7 Resultados Experimentais 140

7.1 Introdução 140

7.2 Descrição do Protótipo Experimental 140

7.3 Resultados Experimentais 144

7.4 Considerações finais do capítulo. 154

8 Conclusões Gerais e Propostas de Continuidade 156

8.1 Conclusões Gerais 156

8.1 Propostas de Continuidade 158

8.2 Publicações 159

Referências 161 


\section{Introdução}

O crescimento da população e da economia mundial tem motivado o aumento no consumo de energia elétrica. De acordo com as previsões do relatório International Energy Outlook 2017, lançado pela Administração de Informação de Energia (EIA) dos EUA, espera-se que o consumo mundial de energia elétrica sofra um aumento em torno de $28 \%$ ao longo dos próximos 22 anos. Assim, pressupõe a melhoria contínua de tecnologias com base nas tendências atuais. Em termos de fontes de geração de energia elétrica, as energias renováveis, possuem uma estimativa de crescimento de $69 \%$, ao passo que no ano de 2040 irão fornecer quota equivalente a produção mundial de carvão, impulsionados pelo aumento do crescimento econômico e, com isso, proporcionando uma melhora na eficiência dos dispositivos de geração e incremento na penetração dessas fontes na rede de distribuição e subtransmissão, sendo umas das contribuições, a diminuição dos dióxidos de carbono - $\mathrm{CO} 2$.

Estima-se que mais de $70 \%$ da energia elétrica dos EUA flui através da eletrônica de potência, segundo dados do Instituto de Pesquisas de Energia Elétrica (EPRI) dos EUA, obtendo um papel fundamental, tão importante quanto os computadores, comunicações e tecnologias da informação (Bose, 2017).

No Brasil, o Governo Federal, por meio da Agência Nacional de Energia Elétrica (ANEEL) e do Ministério de Minas e Energia, a partir do programa PROINFA (Programa de Incentivo às Fontes Alternativas de Energia Elétrica), com a intenção de promover soluções para os problemas de fornecimento de energia elétrica, tem incentivado o uso de sistemas de energias renováveis, tais como os geradores eólicos, os painéis fotovoltaicos, as células de combustível associadas ou não a elementos armazenadores de energia, como baterias e ultracapacitores, dentre outros, configurando, dessa forma, a Geração Distribuída (GD). Nesse contexto, a GD está sendo amplamente difundida e explorada em centros de pesquisas e empresas privadas, principalmente quando operam conectadas à rede de distribuição.

Assim, a utilização da GD pode trazer benefícios ao fornecimento de energia elétrica, tais como: redução de custos, melhoria dos níveis de tensão, redução das perdas de potência em projetos de cogeração, não agressão ao meio ambiente em plantas ecologicamente corretas, dentre outros (El-Khattam; Salama, 2004), além de caracterizarem-se por serem instaladas próximas aos centros consumidores. 
No entanto, se a inserção dessas novas fontes não for feita de forma adequada, podem surgir problemas relacionados ao planejamento (Cataliotti et al., 2008), controle e gerenciamento do sistema elétrico, visto que as mesmas modificam a capacidade de curto-circuito da rede na qual estão inseridas, podendo causar mal funcionamento na coordenação de relés de proteção (Dugan; McDermott, 2002; Campoccia et al., 2003; Chaitusaney; Yokoyama, 2005) além de contribuírem para o aumento das perdas nos condutores elétricos, o que causa uma série de problemas na rede elétrica, como, por exemplo, interferências eletromagnéticas, aumento das taxas das distorções harmônicas de tensão, aquecimento excessivo de transformadores e aumento dos ruídos na frequência audível (Dugan; Mcgranaghan; Beaty, 1996; He et al., 2014), o que contribui para a redução da qualidade da energia elétrica.

Para o estabelecimento de procedimentos relacionados à Qualidade da Energia Elétrica (QEE), como os indicadores de qualidade e a metodologia, relativos à conformidade de tensão em regime permanente e às perturbações na forma de onda da tensão, pode-se mencionar o módulo 8 do PRODIST (Procedimentos de Distribuição de Energia Elétrica no Sistema Elétrico Nacional - 10 ${ }^{\mathrm{a}}$ Revisão) que trata dentre outros assuntos de: fator de potência, harmônicos, variações de frequência, alterações da tensão em regime permanente, variações de tensão de curta duração (VTCD), como os afundamentos (sags) e elevações (swells), bem como desequilíbrios e flutuações (fliker) de tensão.

No entanto, com o intuito de melhorar a QEE na rede elétrica, têm sido propostos diversos tipos de condicionadores de qualidade de energia elétrica dentre os quais é possível citar: Condicionadores Unificados de Qualidade de Energia e os Filtros Ativos de Potência (APF - Active Power Filters) série, paralelo e híbridos, dentre outros (Pigazo et al., 2010; Bhattacharya et al., 1996; Fujita; Akagi, 1998; Aredes; Fernandes, 2009; Kamran; Habetler, 1998; Silva et al., 2002; Nasiri; Amac; Emadi, 2004; Jeon; Cho, 1997; Kwon; Choi; Kim, 2001; Barriviera et al., 2012).

O condicionamento da energia elétrica significa alterar, transformar ou simplesmente adequar suas características para um objetivo específico (Marafão, 2004), que, no caso em questão, resume-se em melhorar a qualidade da tensão disponibilizada às cargas e drenar correntes senoidais da rede dentro dos limites estabelecidos por normas de qualidade de energia (Barriviera et al., 2012). 
Os filtros ativos de potência, série e paralelo, combinados em uma única topologia são conhecidos na literatura como Condicionadores Unificados de Qualidade de Energia (UPQC - Unified Power Quality Conditioner) (Aredes; Häfner; Heumann, 1995; Fugita; Akagi, 1996), e realizam a compensação simultânea tanto das correntes de entrada como das tensões de saída. No caso das topologias convencionais de UPQC, as referências de compensação de tensão e corrente são grandezas não senoidais.

Com o intuito de melhorar o desempenho do UPQC, foi proposto em (Dias et al., 2011; Aredes; Fernandes, 2009) o iUPQC (Unified Power Quality Conditioner with Inverted Topology), ou seja, o condicionador unificado de qualidade de energia com topologia invertida, o qual, na prática, pode-se chamar UPQC dual. Este apresenta várias vantagens em relação ao UPQC convencional, como, por exemplo: as referências de compensação tanto da corrente como da tensão apresentam formas senoidais, o que facilita o projeto dos controladores (Silva et al.; 2011; Barriviera et al., 2012). No UPQC com topologia invertida, o filtro ativo série opera como fonte de corrente senoidal, enquanto o filtro paralelo opera como fonte de tensão senoidal.

Com base nessas informações, e utilizando as normas nacionais e internacionais que estabelecem os limites de harmônicos de tensão e de corrente para as atividades relacionadas ao funcionamento e desempenho dos sistemas de distribuição da energia elétrica, esta pesquisa propõe o estudo, projeto e implementação experimental de um UPQC com topologia invertida, também conhecido como UPQC dual, aplicado em um sistema elétrico monofásico, a fim de analisar seus efeitos conectados no sistema de GD.

Além disso, é foco desta pesquisa a utilização de conversores multiníveis tipo ANPC (Active Neutral Point Clamped) cinco níveis para aplicações em baixas e médias tensões, o desenvolvimento de um algoritmo para determinação das referências de corrente a partir da Teoria da Potência Conservativa (TPC), no qual a premissa visa o aumento na qualidade da energia elétrica. Todos os algoritmos de controle e modulação serão implementados em um microcontrolador de ponto flutuante (MCU) de 32bits, família C2000 da Texas Instruments, modelo TMDSDOCK28379D.

Ademais, os dois conversores estáticos de potência (série e paralelo) realizam as funções de filtros ativos de potência série e paralelo. O conversor série atua como um filtro ativo série de potência, o qual irá operar como uma fonte de corrente senoidal 
em fase com a tensão da rede elétrica. O conversor paralelo atua como um filtro ativo de potência paralelo, o qual irá operar como uma fonte de tensão senoidal, também em fase com a tensão da rede elétrica, fornecendo à carga tensão estabilizada com baixa distorção harmônica (DHT) com a rede elétrica presente (Barriviera et al., 2012). No momento de ausência da rede elétrica, os dois conversores multiníveis são inibidos, ou seja, desligados.

Os modelos matemáticos são implementados em um sistema de coordenadas estacionárias, bem como as análises matemáticas, de modo a obter as funções de transferência que representem os sistemas físicos dos conversores estáticos série e paralelo. A modelagem matemática das malhas de controle da corrente e tensão também são apresentadas. Do mesmo modo é realizado o estudo sobre os fluxos de potência entre os conversores série e paralelo. A fim de comprovar o desenvolvimento teórico e avaliar o desempenho estático e dinâmico de toda a planta, são mostrados os resultados por meio de simulações computacionais e de ensaios experimentais realizados em laboratório.

\subsection{Objetivos}

\subsubsection{Objetivo Geral}

O objetivo deste projeto baseia-se no estudo, análise, controle e implementação experimental de um UPQC, composto por conversores estáticos de potência multiníveis ANPC de cinco níveis aplicados a sistemas elétricos monofásicos com potência nominal de $1 \mathrm{kVA}$, operando conectado à rede de distribuição, realizando a compensação ativa de potência série e paralela.

Os algoritmos para determinação da referência de corrente são desenvolvidos a partir da TPC. O protótipo tem o intuito de realizar o condicionamento ativo de potência simultâneo da corrente de entrada e da tensão da saída, controlados de forma dual aos UPQCs convencionais. Assim, os conversores série e paralelo são sempre gerenciados como fontes senoidais de corrente e de tensão. Dessa forma, as principais contribuições deste trabalho estão relacionadas a análise de estabilidade entre os conversores estáticos de potência, a Teoria da Potência Conservativa para o algoritmo de referência, assim como a descrição detalhada dos conversores 
multiníveis ANPC de cinco níveis em conjunto com a implementação da modulação aplicada em um UPQC.

\subsubsection{Objetivo Específico}

Os objetivos específicos deste trabalho estão definidos abaixo:

- Estudar e implementar a Teoria da Potência Conservativa no UPQC;

- Modelagem matemática do conversor ANPC 5 níveis;

- Implementar o controle e a modulação do conversor multinível em um único microcontrolador de ponto flutuante (MCU) de 32bits;

- Estudar e implementar os conversores estáticos de potência multinível ANPC de 5 níveis;

- Estudar e implementar estratégias de compensação ativa de potência série e paralela em sistemas UPQC;

- Estudar e modelar os conversores estáticos de potência série e paralelo, bem como as malhas de tensão do barramento CC, implementados no UPQC aplicado em sistemas monofásicos;

- Apresentar as análises de estabilidade entre os conversores série e paralelo;

- Apresentar as análises de controle do fluxo de potência ativa entre os conversores série e paralelo;

- Realizar simulações computacionais do sistema UPQC completo;

- Sintetizar corrente e tensão no ponto de acoplamento comum (PAC) com índices de qualidade de energia estabelecidos por normas nacionais e internacionais para equipamentos eletrônicos;

- Realizar testes experimentais e avaliar seus comportamentos estáticos e dinâmicos. 


\subsection{Contribuições do Trabalho}

O presente trabalho visa contribuir com a área de Engenharia Elétrica/Sistemas Dinâmicos/Conversão e Retificação da Energia Elétrica por meio da apresentação de um Sistema UPQC monofásico, com a finalidade de atuar como filtro ativo de potência série e paralelo com controles independentes de tensão na carga e corrente de entrada realizando, assim, a supressão dos harmônicos da tensão da rede elétrica; a compensação da tensão de saída fornecendo tensão senoidal regulada com baixa distorção harmônica em fase com a tensão de entrada; eliminação dos harmônicos de corrente de carga; a correção do fator de potência, tornando a corrente de entrada senoidal, em conformidade com os limites estabelecidos por normas de qualidade de energia; compensação da potência reativa da carga; e a compensação das correntes de cargas conectadas em sistemas elétricos monofásicos. Os algoritmos de geração de referências são implementados a partir da metodologia de projeto baseada na Teoria da Potência Conservativa.

Os conversores estáticos de potência utilizados neste trabalho são os conversores multiníveis tipo 5L-ANPC. Dessa forma, apresenta-se o modelo matemático detalhado, ainda não desenvolvido na literatura, e mostra-se a implementação detalhada da modulação em um único microcontrolador de ponto flutuante (MCU) de 32bits, além da análise de estabilidade entre os conversores estáticos.

Além disso, a estratégia de compensação convencional, na qual o conversor série funciona como fonte de tensão não senoidal e o conversor paralelo como fonte de corrente não senoidal, não serão avaliados neste trabalho. A estratégia de compensação aplicada neste trabalho é a dual, ou seja, o conversor série produz corrente senoidal, enquanto que, o conversor paralelo tensão senoidal. Em (Barriviera et al., 2011) é mostrado uma análise entre as vantagens e desvantagens da compensação ativa de potência série e paralela nos dois modos de controle dos conversores aplicados em um UPS Line Interactive Monofásico. 


\subsection{Organização do Trabalho}

Este trabalho é organizado da seguinte forma:

No capítulo 1 é apresentada a introdução da pesquisa, objetivos gerais e específicos, contribuição do trabalho, bem como a organização dos capítulos.

No capítulo 2, é realizada uma revisão bibliográfica sobre o tema, além de discutir os principais tipos de conversores multiníveis, bem como os tipos de compensações de distúrbios.

No capítulo 3, o conversor multinível ANPC cinco (5) níveis é apresentado, bem como a modulação utilizada, o modelo matemático e simulações computacionais.

No capítulo 4, o fundamento matemático necessário para desenvolver os algoritmos de referências utilizados neste trabalho é apresentado. Aborda-se a TPC, uma modelagem matemática dos circuitos elétricos nos quais são mostradas as decomposições da corrente e potência e que permitem determinar qual é a demanda da compensação.

No capítulo 5, é apresentada a modelagem matemática dos conversores estáticos de potência série e paralelo e do elo CC, bem como a modelagem matemática das malhas de controle de corrente, tensão e da tensão do elo CC, além do fluxo de potência por meio dos conversores e análise de estabilidade.

No capítulo 6, são mostrados os resultados obtidos pelas simulações computacionais, no qual o UPQC opera de forma estática e dinâmica, realizando a compensação dos distúrbios da rede elétrica.

No capítulo 7, são mostrados os resultados obtidos a partir de ensaios experimentais, conduzidos em laboratório, do protótipo completo funcionando em malha fechada conectado na rede elétrica, operando de forma estática e dinâmica.

No capítulo 8, apresentam-se as conclusões, bem como as propostas de continuidade deste trabalho. 


\section{Revisão Bibliográfica}

\subsection{Introdução}

Este capítulo apresenta o UPQC monofásico com compensação ativa de potência, série e paralela, o seu princípio de funcionamento e suas principais características, em termos da estratégia de controle dual utilizada na compensação ativa de potência. Além disso, são descritos os principais tipos de estruturas de potência, como os Conversores Multiníveis mais utilizados em aplicações envolvendo equipamentos eletrônicos em vários níveis de potência, abrangendo suas vantagens e desvantagens, bem como o Conversor Multinível 5L-ANPC utilizado neste trabalho e a modulação aplicada nos conversores estáticos de potência.

\subsection{Condicionador de Qualidade de Energia Unificado (UPQC)}

O conversor sugerido é apresentado conforme o diagrama em blocos na Figura 2.1, o qual é composto por um conversor série chamado de Filtro Ativo de Potência Série (FAPS) e um paralelo chamado de Filtro Ativo de Potência Paralelo (FAPP), combinados em uma única estrutura, interligados por um elo CC comum a eles, chamado de conversores de potência back-to-back, bem como Delta-Conversion.

Figura 2.1 - Diagrama em blocos simplificado de um sistema UPQC.

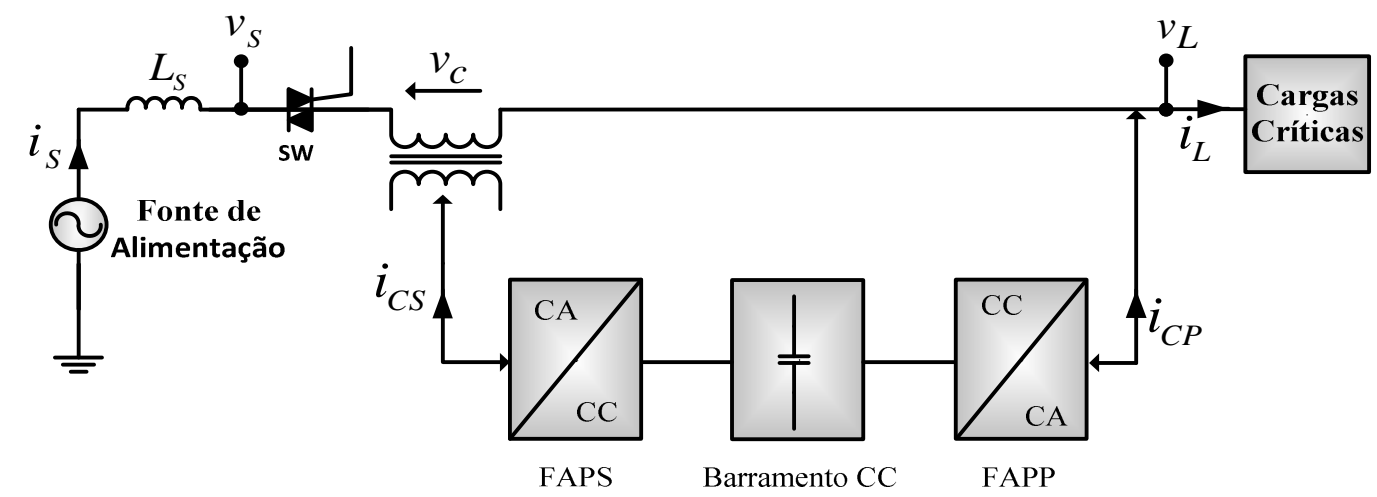

Fonte: Dados da pesquisa do autor. 
Os FAPS com compensação convencional são comumente utilizados para regular e balancear as tensões fornecidas à carga e eliminar distorções na forma de onda da tensão. A conexão é realizada em série por meio de um transformador de acoplamento entre a fonte e a carga. A tensão de alimentação é somada às componentes de compensação com as mesmas amplitudes, mas em fases opostas, com uma parcela fundamental cujo objetivo é manter as tensões de saída equilibradas e reguladas.

Já os FAPP com compensação convencional são utilizados para compensar reativos de cargas não lineares e eliminar/reduzir os harmônicos. A conexão é realizada em paralelo com carga, conectada diretamente no ponto de acoplamento comum. As correntes de compensação com fases opostas e com as mesmas amplitudes são injetadas a fim de eliminar as componentes indesejadas, com o objetivo de realizar a compensação de reativos e a supressão das correntes harmônicas.

Em (Moran, 1989) foi apresentado um trabalho com o objetivo de realizar o condicionamento simultâneo da corrente de entrada e da tensão de saída em redes monofásicas. Depois de alguns anos foram apresentados resultados experimentais para UPQCs trifásicas a três fios (Fujita; Akagi, 1996), que ficaram conhecidos e despertaram o interesse de vários pesquisados e centros de pesquisas, a fim de aplicar esses tipos de equipamentos em redes elétricas em diferentes níveis de tensões. Com isso, um grande número de trabalhos foram e continuam sendo apresentados, com diversos tipos de topologias, algoritmos de compensação, diferentes controladores, dentre outros (Aredes et al., 1998; Khadkikar et al., 2010; Vadirajacharya et al., 2011; Ucar et al., 2011; Khadkilar, 2012; Teke et al., 2013; Karanki et al., 2013).

Nesse sentido, a estratégia de controle dual foi apresentada como uma possibilidade para melhorar a eficiência das UPQCs (Aredes; Fernandes, 2009; Santos et al., 2011; Santos et al., 2014; Aredes et al., 2014; Modesto et al., 2015). Portanto, neste trabalho será utilizado esta mesma estratégia de controle dual.

A partir da estratégia de controle dual utilizada, o conversor estático de potência série atua como uma fonte de corrente senoidal, cujo intuito é tornar as correntes senoidais, em fase, e equilibradas com a parcela da componente fundamental da tensão da rede elétrica. Assim, têm-se um caminho de alta impedância para as correntes harmônicas geradas por cargas não lineares, isolando-as. As amplitudes 
das correntes senoidais dependem da demanda de potência ativa da carga e às perdas da estrutura física (Silva et al., 2002; Barriviera et al., 2012).

Já o conversor estático de potência paralelo atua como uma fonte de tensão senoidal, cujo intuito é impor/estabelecer tensões em fase, equilibradas e reguladas com baixo conteúdo harmônico, com as tensões da rede elétrica. Assim, realiza-se um caminho de baixa impedância para as correntes harmônicas das cargas, ou seja, os harmônicos que aparecem nas correntes das cargas circulam por meio do conversor estático de potência paralelo por oferecer um caminho de baixa impedância para esses harmônicos (Silva et al., 2002; Barriviera et al., 2012).

A partir do momento em que se têm impedâncias elevadas e baixas conforme descrito acima nos conversores, série e paralelo, as correntes harmônicas das cargas fluem de forma natural por meio do conversor paralelo de tensão. De forma a assegurar a compensação das potências reativas nos conversores série, faz-se importante o controle das referências em fase com as respectivas tensões da rede elétrica (Modesto et al., 2015).

As tensões de saída do conversor paralelo de tensão são controladas para seguir referências senoidais e equilibradas, realizando assim as compensações dos harmônicos e desequilíbrios de tensão, de tal forma que as diferenças de amplitude entre as tensões de entrada e de saída surgem nos transformadores série, ou seja, quando se tem tensões na entrada do sistema com perturbações e no conversor paralelo de tensão são impostas tensões senoidais, a diferença entre as duas tensões são os harmônicos e desequilíbrios de tensão que surgem sobre o transformador série. Desse modo, os transformadores série de acoplamento absorvem de maneira natural as perturbações presentes nas tensões do sistema elétrico (Modesto et al., 2015).

Além disso, no momento em que a rede elétrica não está presente, a chave estática instalada na entrada do equipamento é posicionada na posição "aberta", e ambos os conversores estáticos de potência são inibidos, uma vez que os mesmos não produzem energia, isto é, eles somente processam correntes e tensões com harmônicos, distorções, dentre outros, e devem estar sincronizados com a rede elétrica por meio de um algoritmo de sincronismo. No retorno da rede elétrica em condições normais de operação, é realizado o sincronismo, a chave estática é fechada e ambos os conversores são habilitados. 
O UPQC com estratégia de controle dual apresenta algumas características e vantagens, quando comparadas com a estratégia convencional, como conforme (Barriviera et al., 2011):

- Atua como filtro ativo de potência, série e paralelo, com controles independentes de corrente de entrada e tensão de saída;

- Eliminação dos harmônicos de tensão de entrada, bem como regulada e com baixo conteúdo harmônico para a alimentação da carga;

- Torna a corrente de entrada senoidal e com baixa DHT, a fim de corrigir o fator de potência e eliminar os harmônicos de corrente da carga;

- Referências de corrente e tensão são senoidais, de forma a contribuir com os conversores de potência a imporem/aplicar suas referências com mais facilidade;

- Não necessita de malhas adicionais de controle para compensar afundamentos e elevações de tensão;

- Comportamento estático e dinâmico superior em relação à estratégia convencional.

Neste contexto, alguns trabalhos apresentados na literatura trazem algumas aplicações envolvendo sistemas UPS/UPQC com as mesmas características construtivas, com a diferença de ter acoplado ao elo CC um banco de baterias, bem como aplicados em sistemas monofásicos e trifásicos, com estratégias de compensação convencional (Bhattacharya et al., 1991; Kamran; Habetler, 1995) e com estratégias de compensação dual (Silva et al., 2002; Barriviera et al., 2012; Modesto et al., 2015). No trabalho apresentado por (Jeon; Cho, 1997), o conversor série pode ser dimensionado para uma potência entre 20 e $25 \%$ da potência nominal da carga.

É importante ressaltar que foi realizada uma análise comparativa entre as estratégias de compensação convencional e dual em algumas topologias de UPS/UPQC trifásicas compostas por dois conversores estáticos de potência série e paralelo, sendo: Split-Capacitor (Silva et al., 2002; Silva; Modesto, 2005; Santos et al., 2014); Four-Leg (Ucar; Ozdemir, 2013); Three-Leg no conversor série e um Four-Leg no conversor paralelo (Khadkikar; Chandra, 2009; Silva et al., 2011; Modesto et al., 2015). 


\subsection{Conversores Multiníveis}

Como a maioria dos processos industriais e as concessionárias de energia buscam formas para aumentar a eficiência e reduzir os custos de produção a partir de mudanças ou aumento das instalações e das potências de máquinas e equipamentos, faz-se necessário utilizar novas tecnologias disponíveis no mercado no qual foram desenvolvidos em centros de pesquisas situados em empresas privadas ou centros acadêmicos.

Podem-se citar algumas tecnologias disponíveis no mercado como, o uso de semicondutores de alta tensão, com capacidades de bloqueio de tensão de $3300 \mathrm{~V}$, $4500 \mathrm{~V}$ e $6500 \mathrm{~V}$, sendo que em alguns casos torna-se difícil encontrar semicondutores que operem em altas correntes (50 A até $3600 \mathrm{~A}$ ) e altas frequências. No entanto, umas das tecnologias atualmente disponíveis, são os conversores multiníveis, no qual a característica é a redução da tensão nos semicondutores, ou seja, a utilização de uma quantidade maior de dispositivos eletrônicos com menor tensão, sendo que são encontradas com mais facilidade no mercado (Franquelo et al., 2008; Rodriguez et al., 2010; Lima et al., 2011).

Assim, é possível conectar diretamente os conversores estáticos de potência a redes elétricas de média tensão, bem como reduzir os harmônicos de linha. De modo geral, sua estrutura, sintetiza tensões senoidais a partir de vários níveis de tensão, tipicamente obtidos de fontes de tensão contínua. Essa característica possibilita o uso de modulações com dois ou mais níveis de tensão impostas à carga, de tal modo que permite a redução do volume dos elementos de filtragem (Lima et al., 2011), constituindo, assim, uma vantagem em relação às estruturas de conversores tradicionais. Com isso, melhora-se a qualidade da tensão de saída, com nível de esforço reduzido dos semicondutores em médias tensões e com acréscimo de potência.

A seguir são apresentadas as topologias mais utilizadas em conversores multiníveis. 


\subsubsection{Ponto Neutro Grampeado a Diodo (Neutral Point Clamped - NPC)}

Os conversores multiníveis tipo NPC de três níveis foram apresentados por (Backer; Bedford, 1979, 1980) e depois por (Nabae; TakaHashi; Akagi, 1981), sendo comercializados por Empresas como $A B B$ e Converteam, dentre outras, e podem ser visualizados na Figura 2.2 por meio do esquema simplificado por fase.

O conversor NPC foi difundido em diversas aplicações, a partir de sua simplicidade e de sua capacidade de condicionar formas de onda com mais níveis quando comparado aos conversores tradicionais de dois níveis.

A estrutura monofásica é composta por 2(n-1) chaves semicondutoras de potência, diodos de grampeamento por fase que, normalmente, devem suportar as mesmas tensões suportadas pelas chaves, e $n-1$ capacitores conectados entre 0 ponto central no barramento de corrente contínua (CC), sendo $n$ o número de níveis. A tensão sobre os capacitores é dada por $V c c /(n-1)$, de forma a grampear a tensão máxima sobre cada semicondutor com o mesmo valor. Assim, a condução dos diodos garante que a tensão sobre cada uma delas seja igual a tensão dos capacitores, conforme apresentado na Figura 2.2.

Os semicondutores $S_{a 1}$ e $S_{a 3}$ não podem conduzir simultaneamente, assim como as chaves $S_{a 2}$ e $S_{a 4}$. Os níveis de tensão na carga são: $V_{c d} / 2,0,-V_{c d} 2$. Além do que, o número de níveis da tensão de fase é inferior ao da tensão de linha, ou seja, a tensão de linha apresenta cinco níveis distintos. $V_{c c}, V_{c c} / 2,0,-V_{c c} / 2 \mathrm{e}-V_{c c}$.

Figura 2.2 - Conversor NPC três níveis.

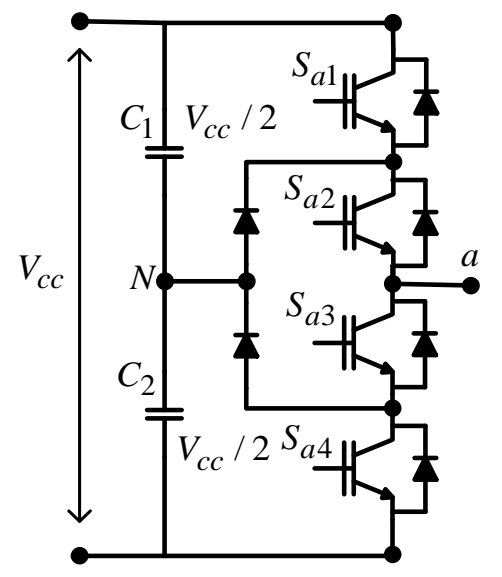

Fonte: Dados da pesquisa do autor. 
As vantagens desta estrutura são: quantidade reduzida de semicondutores; redução do número de capacitores; conversor modular; elo CC comum; quanto maior número de níveis, menor será a taxa de distorção harmônica na saída; não necessidade de isolamento da fonte de tensão CC (Lai; Peng, 1996; Rodriguez et al., 2010).

No entanto, como desvantagem, possuem: perdas relativamente altas de comutação, que limitam a frequência de comutação (Sayago; Brückner; Bernet, 2008); perda assimétrica nos semicondutores (Brückner; Bernet, 2001; Brückner; Bernet; Güldner, 2005; Brückner; Bernet; Steimer, 2007; Sayago; Brückner; Bernet, 2008); elevado número de semicondutores quando submetidos a grandes níveis de tensão; aparecimento de elevadas tensões de bloqueio sobre os diodos de grampeamento; desbalanceamento de tensão nos capacitores do barramento; grampeamento indireto dos semicondutores e diodos; e dificuldade de expansão para aplicações com maior número de níveis (Choi et al., 1991; Steimer et al., 2005; Grigoletto et al., 2009).

No início da utilização das topologias NPC, houve uma forte crítica sobre o equilíbrio nas tensões dos capacitores do elo CC. Este tópico tem sido alvo de muitas pesquisas em equipamentos de alta potência. Portanto, este problema foi resolvido utilizando a capacidade de redundância dos inversores NPC. Assim, pesquisas importantes têm sido realizadas para equilibrar as tensões nos capacitores do elo CC em diversas estratégias (Bouhali et al., 2007; Tallam; Naik; Nondahl, 2005; Pan et al., 2005; Pan; Peng, 2006; Holtz; Oikonomou, 2007; Zaragoza et al., 2009).

As estratégias de modulação aplicadas neste tipo de conversor multinível são: modulação por largura de pulso (PWM), modulação vetorial espacial (SVM) e eliminação seletiva de harmônicos (SHE), dentre outras (Zaragoza et al., 2009).

\subsubsection{Capacitor Flutuante (Flying Capacitor - FC)}

Os conversores multiníveis com capacitor flutuante foram apresentados por (Meynard; Foch, 1992), sendo posteriormente produzidos e comercializados por diversas empresas como a Alstom, dentre outras. O esquema monofásico desses conversores, simplificado para três níveis, é mostrado por meio da Figura 2.3. Conforme pode ser observado, a estrutura apresentada é similar ao conversor 
multinível NPC, com a exceção de empregar capacitores de grampeamento, ao invés de diodos de grampeamento.

Sua estrutura de três níveis monofásica é composta por $n-1$ capacitores no elo CC, $n-1$ grupo de chaves semicondutoras, de forma que cada grupo é composto por um par de semicondutores ativos e $n$-2 capacitores de grampeamento, em que $n$ é o número de níveis (Serpa, 2007).

Os semicondutores $S_{a 1}$ e $S_{a 4}$ não podem conduzir simultaneamente, assim como $S_{a 2}$ e $S_{a 3}$. Os níveis de tensão na carga são: $V_{c d} / 2,0,-V_{c d}$, conforme apresentado na Figura 2.3. Ao realizar a expansão do número de níveis deste conversor FC, tem-se o aumento no número de capacitores (Peng, 2001), ao ponto que cresce o número de capacitores de forma exponencial com o número de níveis do conversor.

Figura 2.3 - Conversor Capacitor Flutuante - três níveis.

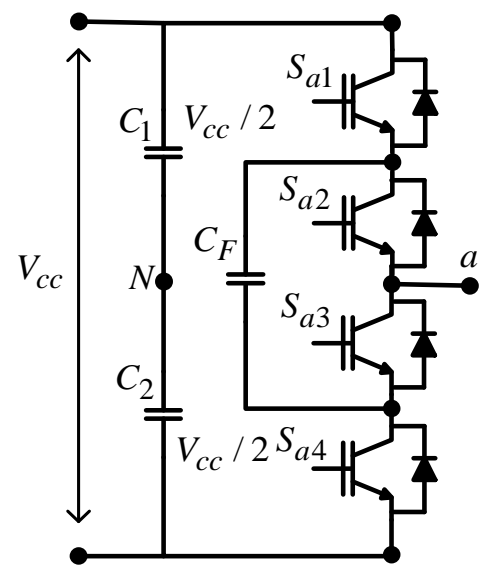

Fonte: Dados da pesquisa do autor.

As vantagens da estrutura são: grande quantidade de capacitores de armazenamento fornece energia extra em casos de quedas de energia; as combinações redundantes das chaves estáticas para equilibrar os níveis de tensão diferentes; fluxo de potência ativa e reativa podem ser controlados, assim utilizado em transmissão de alta tensão e em correntes contínuas (Lai; Peng, 1996).

Entretanto, esta estrutura apresenta algumas desvantagens como: o número de capacitores de armazenamento necessários é maior quando o número de níveis de tensão é elevado, ou seja, quanto maior o número de níveis, maior a quantidade de capacitores requerida. Tornando o custo da estrutura elevado, aumentando seu peso e fazendo que a mesma seja volumosa; o controle do inversor é mais complexo; 
e a frequência e as perdas de comutação são maiores para altas taxas de transmissão de energia (Lai; Peng, 1996; Rashid, 2015).

As estratégias de modulação aplicadas neste tipo de conversor multinível são: modulação por largura de pulso (PWM), modulação vetorial espacial (SVM), eliminação seletiva de harmônicos (SHE), fase deslocada (Phase Shifted - PS), dentre outras (Zaragoza et al., 2009; Colak et al., 2010).

\subsubsection{Inversores em Cascata com separação de Fontes de Corrente Contínua (Cascated Inverters)}

Os conversores multiníveis conhecidos como inversores em cascata (Cascated Inverters), e também como Ponte-H em Cascata (Cascated H-Bridge - CHB), foram exibidos por (Baker; Bannister, 1975) e, posteriormente, por (Lai; Peng, 1996), sendo fornecidos/comercializados por diversas empresas, como, por exemplo, pela Siemens e pela ASIRobicon, dentre outras. A Figura 2.4 mostra o esquema monofásico, simplificado para três níveis, de tais conversores. Vale ressaltar que em alguns casos, esta topologia também é conhecida como fontes de tensão separadas.

Figura 2.4 - Inversor em Cascata (Castated Inverters) três níveis.

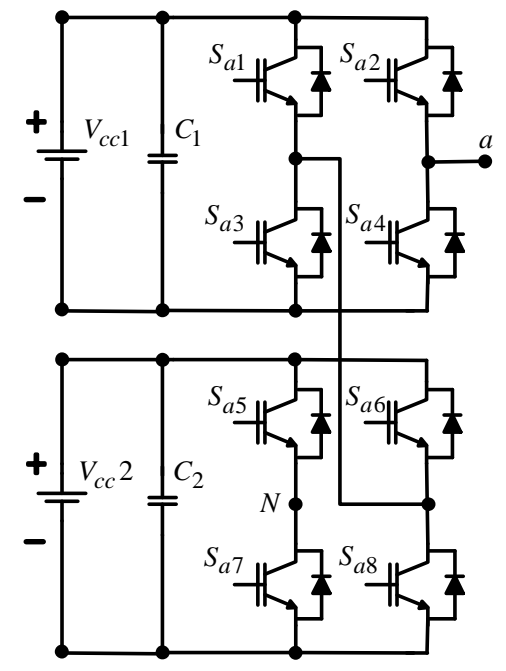

Fonte: Dados da pesquisa do autor.

Esta estrutura consiste em uma associação de conversores de potência convencionais de baixa tensão, baseada em conversores monofásicos em ponte completa, em cascata de dois níveis, e é largamente empregada para alcançar 
tensões com vários níveis. A tensão de saída é condicionada ao somatório das tensões de saída de cada respectivo módulo. Sendo assim, os módulos com cada conversor convencional são alocados em série, a fim de reproduzir a tensão na saída.

Como vantagens, nesta estrutura não há a necessidade de diodos de fixação ou de capacitores de balanceamento das tensões no elo CC e entre os conversores de vários níveis para atingir o mesmo número de níveis de tensão, o que reduz custos, manutenção e complexidade; a disposição de estruturas de conversores estáticos de potência são modulares e de mesmo nível; o controle é simples e podem ser utilizadas comutações suaves; (Lai; Peng, 1996). Dentre suas desvantagens pode-se citar: necessidade de fontes de corrente contínua separadas para os conversores estáticos de potência (Lai; Peng, 1996; Rashid, 2015).

As estratégias de modulação aplicadas neste tipo de conversor multinível são: modulação por largura de pulso (PWM), modulação vetorial espacial (SVM), eliminação seletiva de harmônicos (SHE) e fase deslocada (Phase Shifted - PS), dentre outras (Zaragoza et al., 2009; Colak et al., 2010).

\subsubsection{Inversor com Grampeamento Ativo do Neutro (Active Neutral Point Clamped - ANPC) - 3 níveis}

Os conversores multiníveis tipo Active Neutral Point Clamped (ANPC) de três níveis foram apresentados por (Brückner; Bernet, 2001), sendo comercializados por Empresas como ABB, Siemens, Allen Bradley, Converteam, dentre outras, conforme o esquema simplificado por fase mostrado na Figura 2.5. Esta estrutura foi desenvolvida de forma a melhorar a principal desvantagem do conversor multinível NPC, na qual é realizada a distribuição assimétrica das perdas nas chaves semicondutoras de potência, de tal modo a se obter o balanceamento e a redução da temperatura de junção dos semicondutores (Brückner et al., 2005 e 2007; Floricau et al., 2008; Li et al., 2009). Além disso, não há a necessidade de utilizar dispositivos eletrônicos de alto custo. 
Figura 2.5 - Active Neutral Point Clamped (ANPC) - 3 níveis monofásico.

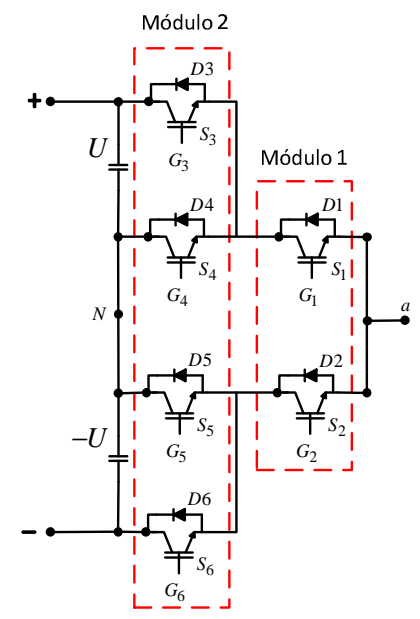

Fonte: Dados da pesquisa do autor.

Esta estrutura proporciona um aumento na taxa de potência de saída em $20 \%$ (Brückner; Bernet, 2001) em relação à estrutura NPC. Os pontos de operação mais críticos que determinam a corrente máxima de saída ocorrem com índice de modulação máximo $m=1,155$ com fator de potência igual a um (Rodriguez et al., 2010).

Considerando um conversor multinível ANPC três níveis em uma topologia monofásica, têm-se basicamente quatro estados de comutação para conectar o terminal de saída (corrente alternada) ao ponto médio (ponto neutro) do elo CC, sendo dois pela parte superior e dois pela parte inferior do conversor.

Ao ligar os semicondutores S4 e S1, a corrente de fase é conduzida pelo caminho superior ao ponto neutro em ambas as direções. Da mesma maneira, ao ligar S5 e S2, a corrente de fase é conduzida pelo caminho inferior do conversor ao ponto neutro em ambas as direções. No momento em que S4 e S1 estão desligadas, S3 pode estar ligada ou desligada, o mesmo pode ser aplicado para S6 na qual pode estar ligada ou desligada, no momento em que S5 e S2 estão desligadas. Os resultantes dos quatros estados de chaveamento do ponto zero estão descriminados como 0U2, OU1, OL1 e 0L2, mostrados na Tabela 2.1.

Portanto, a distribuição das perdas de condução durante as passagens por zero pode ser controlada através da escolha dos caminhos superiores e inferiores. No estado positivo, S5 está ligado para garantir a tensão igual entre S2 e S6, igualmente como S4 é ligado no estado negativo para garantir a tensão igual entre S3 e S1, de 
tal forma realizando o balanceamento entre os semicondutores (Rodriguez et al., 2010). A sequência de chaveamento está mostrada na Tabela 2.1.

Tabela 2.1 - Sequência de chaveamento - ANPC - três níveis.

\begin{tabular}{l|c|c|c|c|c|c}
\hline & $\mathrm{S}_{3}$ & $\mathrm{~S}_{1}$ & $\mathrm{~S}_{2}$ & $\mathrm{~S}_{6}$ & $\mathrm{~S}_{4}$ & $\mathrm{~S}_{5}$ \\
\hline Positivo & 1 & 1 & 0 & 0 & 0 & 1 \\
\hline OU2 & 0 & 1 & 0 & 0 & 1 & 0 \\
\hline OU1 & 0 & 1 & 0 & 1 & 1 & 0 \\
\hline OL1 & 1 & 0 & 1 & 0 & 0 & 1 \\
\hline OL2 & 0 & 0 & 1 & 0 & 0 & 1 \\
\hline Negativo & 0 & 0 & 1 & 1 & 1 & 0 \\
\hline
\end{tabular}

Fonte: Dados da pesquisa do autor.

A fim de diminuir as perdas por comutação, faz-se necessário o uso das comutações a partir dos estados zero. Todas as comutações ocorrem entre um semicondutor ativo e um diodo. Caso tenha mais de dois dispositivos ligados ou desligados, somente o semicondutor e o diodo ativo vão ter perdas por comutação. (Brückner; Bernet, 2001; Rodriguez et al., 2010).

$\mathrm{Na}$ Tabela 2.2, são apresentadas as sequências de comutação de todas as condições operacionais, tanto para corrente acima de zero, como também para corrente abaixo de zero (Brückner; Bernet, 2001; Rodriguez et al., 2010).

Tabela 2.2 - Sequência de comutação ANPC três níveis.

\begin{tabular}{|c|c|c|c|c|c|c|c|c|c|c|c|c|}
\hline & $\mathrm{S}_{3}$ & $D_{3}$ & $S_{1}$ & $D_{1}$ & $\mathrm{~S}_{2}$ & $D_{2}$ & $\mathrm{~S}_{6}$ & $D_{6}$ & $\mathrm{~S}_{4}$ & $\mathrm{D}_{4}$ & $\mathrm{~S}_{5}$ & $\mathrm{D}_{5}$ \\
\hline \multicolumn{13}{|c|}{ Corrente de fase positiva } \\
\hline$+\leftarrow \rightarrow 0 \cup 2$ & $x$ & & & & & & & & & $X$ & & \\
\hline$+\leftarrow \rightarrow$ 0U1 & $X$ & & & & & & & & & $\mathrm{X}$ & & \\
\hline$+\leftarrow \rightarrow 0 \mathrm{~L} 1$ & & & $\mathrm{X}$ & & & $\mathrm{X}$ & & & & & & \\
\hline$+\leftarrow \rightarrow$ 0L2 & $x$ & & & & & $\mathrm{X}$ & & & & & & \\
\hline 0U2 $\leftarrow \rightarrow-$ & & & $X$ & & & & & $\mathrm{X}$ & & & & \\
\hline 0U1 $\leftarrow \rightarrow-$ & & & $\mathrm{X}$ & & & $X$ & & & & & & \\
\hline 0L1 $\leftarrow \rightarrow-$ & & & & & & & & $X$ & & & $X$ & \\
\hline $0 \mathrm{~L} 2 \leftarrow \rightarrow-$ & & & & & & & & $\mathrm{X}$ & & & $x$ & \\
\hline \multicolumn{13}{|c|}{ Corrente de fase negativa } \\
\hline$+\leftarrow \rightarrow 0 \cup 2$ & & $X$ & & & & & & & $x$ & & & \\
\hline$+\leftarrow \rightarrow 0 \mathrm{U} 1$ & & $X$ & & & & & & & $\mathrm{X}$ & & & \\
\hline$+\leftarrow \rightarrow 0 \mathrm{~L} 1$ & & & & $X$ & $X$ & & & & & & & \\
\hline$+\leftarrow \rightarrow$ 0L2 & & $X$ & & & $\mathrm{X}$ & & & & & & & \\
\hline $0 \cup 2 \leftarrow \rightarrow-$ & & & & $\mathrm{X}$ & & & $\mathrm{X}$ & & & & & \\
\hline 0U1 $\leftarrow \rightarrow-$ & & & & $\mathrm{X}$ & $x$ & & & & & & & \\
\hline 0L1 $\nrightarrow-$ & & & & & & & $X$ & & & & & $X$ \\
\hline $0 L 2 \leftarrow \rightarrow-$ & & & & & & & $\mathrm{X}$ & & & & & $\mathrm{X}$ \\
\hline
\end{tabular}

Fonte: Dados da pesquisa do autor. 
As estratégias de modulação aplicadas neste tipo de conversor multinível são: modulação por largura de pulso (PWM), modulação vetorial espacial (SVM), eliminação seletiva de harmônicos (SHE), dentre outras (Barbosa et al., 2005; Floricau et al., 2008 e 2009; Zaragoza et al., 2009; Li et al., 2009; Malinowski et al., 2009; Colak et al., 2010) e Level shifed (McGrath et al., 2000).

\subsection{Topologia adotada}

O trabalho é composto por um Condicionador Unificado de Qualidade de Energia (UPQC) monofásico, com estratégia dual de controle, utilizada na compensação ativa de potência. O UPQC consiste em dois conversores multiníveis estáticos de potência ANPC cinco níveis, combinados em uma única estrutura, interligados por um elo CC. Sendo assim, têm-se um conversor série de potência, atua como fonte de corrente senoidal, impondo correntes senoidais; e um conversor paralelo de tensão, que atua como fonte de tensão senoidal, impondo tensões senoidais na carga. A modulação utilizada em ambos os conversores é a Level Shitfed (McGrath et al., 2000). Ambos os conversores série e paralelo apresentam controles independentes. A Figura 2.6 mostra a topologia proposta completa. 
Figura 2.6 - Topologia adotada

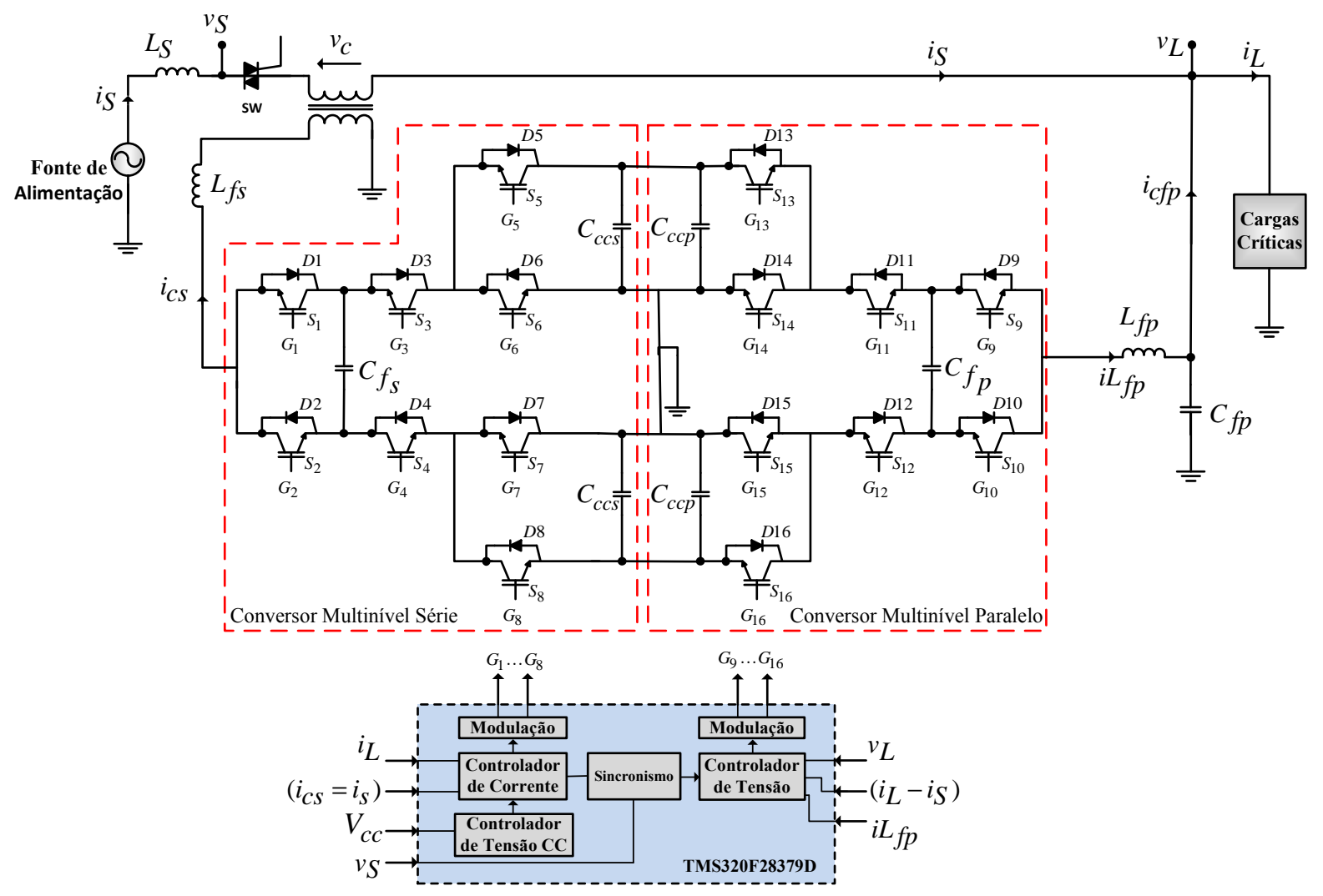

Fonte: Dados da pesquisa do autor.

\subsection{Considerações finais do capítulo}

Este capítulo apresentou a topologia do UPQC monofásico com compensação ativa de potência, série e paralela, o seu princípio de funcionamento, as suas principais características quanto à estratégia de controle convencional e dual utilizada na compensação ativa de potência, e a modulação aplicada aos conversores estáticos de potência.

Também foram apresentados os tipos de estruturas de potência, como os Conversores Multiníveis: Ponto Neutro Grampeado a Diodo (Neutral Point Clamped NPC); Capacitor Flutuante (Flying Capacitor - FC); Inversores em Cascata (Cascated Inverters) com separação de Fontes de Corrente Contínua; Inversor com Grampeamento Ativo do Neutro (Active Neutral Point Clamped - ANPC) - 3 níveis, abrangendo o princípio de funcionamento e suas características. 


\section{Inversor com Grampeamento Ativo do Neutro (Active Neutral Point Clamped -} ANPC) -5 níveis

\subsection{Introdução}

Este capítulo apresenta os detalhes do Inversor ANPC de cinco níveis, como modelo matemático, modulação empregada e implementada, bem como a comparação entre o modelo chaveado e o teórico.

\subsection{Inversor ANPC 5 níveis (5L-ANPC)}

Os conversores multiníveis tipo Active Neutral Point Clamped (ANPC) de cinco níveis foram apresentados por (Brückner et al., 2005), sendo comercializados também por Empresas como ABB, Siemens, Allen Bradley, Converteam, dentre outras. O esquema básico desses conversores é mostrado por meio da Figura 3.1, na representação por fase.

Esta estrutura é uma continuidade do conversor ANPC de três níveis, no qual foi inserido um capacitor de fase e mais dois semicondutores de potência na saída do conversor, realizando, assim, o aumento do número de níveis de três para cinco. Dessa maneira, destaca-se que é possível aumentar ainda mais o número de níveis deste tipo de estrutura (Barbosa et al., 2005). Vale ressaltar ainda que este conversor é a união entre o conversor ANPC três níveis (Figura 3.1 - Módulo 3) e o conversor Capacitor Flutuante (FC) de três níveis (Figura 3.1 - Módulo 1 e 2).

Contudo, o inversor de três níveis oferece maior peso e volume comparado com os inversores de cinco níveis. Isso ocorre devido ao fato do filtro de saída ficar submetido somente à frequência de comutação dos semicondutores de potência.

Em algumas aplicações, utilizam-se chaves em série no Módulo 3, S5 a S8, com o intuito de dividir a tensão de forma uniforme, a fim de aumentar a tensão e, consequentemente, a potência de saída, sendo o mesmo sinal de ativação para ambas (Barbosa et al., 2005). 
Figura 3.1 - Active Neutral Point Clamped (ANPC) - 5 níveis monofásico.

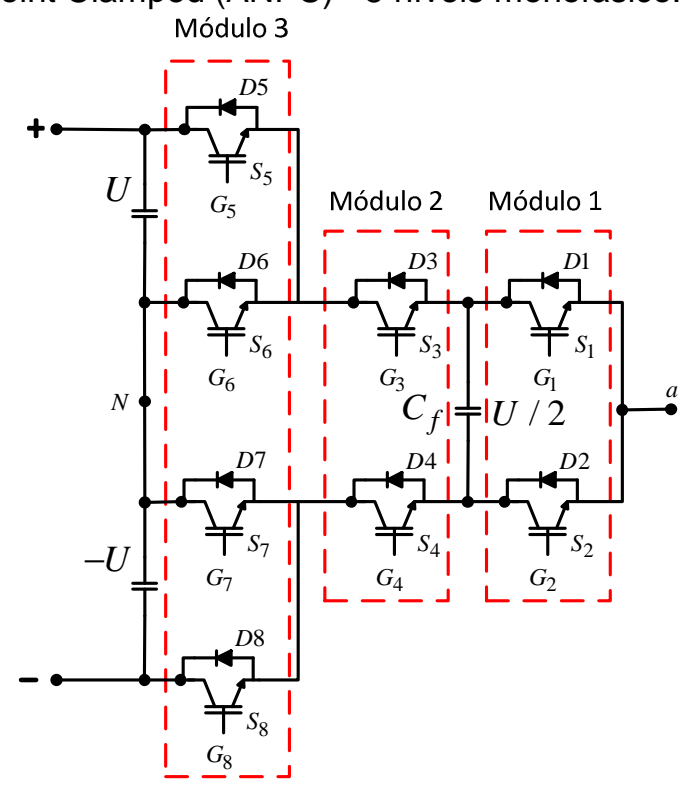

Fonte: Dados da pesquisa do autor.

Neste caso, no conversor multinível ANPC cinco níveis monofásico, têm-se, fundamentalmente, dois estados de comutação para conectar o terminal de saída (corrente alternada) ao ponto médio (ponto neutro) do elo $\mathrm{CC}$, um estado positivo $\mathrm{U}$, um estado negativo $-\mathrm{U}$, dois estados $-\mathrm{U}+\mathrm{U} / 2$ e $\mathrm{U}-\mathrm{U} / 2$ no qual $\mathrm{U} / 2$ representa a tensão sobre o capacitor de fase quando está descarregado ou carregado e dois estados U/2 e $-\mathrm{U} / 2$. A tensão sobre 0 capacitor de fase $C_{f}$ varia entre $-\mathrm{U} / 2$ e $\mathrm{U} / 2$, conforme mostrado na Tabela 3.1.

O índice de modulação é normalizado para a metade da tensão do barramento CC, ou seja, $U / 2$, de tal forma que o índice de modulação é responsável pelo equilíbrio da tensão nos terminais do capacitor de fase (flutuante) e limita a frequência de comutação das chaves S5 a S8, para a frequência fundamental da tensão de saída. $\mathrm{Na}$ Tabela 3.1, são apresentados todos os estados das chaves em que possuem o impacto no capacitor de fase.

A tensão de fase assume cinco níveis de tensão entre o ponto neutro central e ponto de saída: $-U,-U / 2,0, U / 2$ e $U$, no qual são numerados entre 0 e 4 , respectivamente. De acordo com esses níveis, para a geração dos pulsos das chaves, é necessário obter a tensão sobre o capacitor de fase (flutuante) $V_{C f}$, a corrente de saída $i_{C f}$ e a tensão de referência $V_{\operatorname{ref}\{R, S, T\}}$, para equilibrar de forma ativa a tensão sobre os capacitores de fase (Barbosa et al., 2005), constituindo em decidir qual estado de chaveamento será realizado de acordo com as oito chaves. 
Tabela 3.1 - Estado das chaves semicondutoras e o impacto do capacitor de fase (flutuante) - ANPC cinco níveis (Barbosa et al., 2005).

\begin{tabular}{|c|c|c|c|c|c|c|c|c|c|c|c|c|}
\hline \multicolumn{4}{|c|}{ Módulo 3} & \multicolumn{2}{|c|}{ Módulo 2} & \multicolumn{2}{|c|}{ Módulo 1} & \multirow{2}{*}{$\begin{array}{l}\text { Módulos } \\
\text { de } \\
\text { Tensão }\end{array}$} & \multirow{2}{*}{$\begin{array}{l}\text { Tensão } \\
\text { de Fase }\end{array}$} & \multicolumn{2}{|c|}{ Efeito do $\mathrm{C}_{\mathrm{f}}$} & \multirow{2}{*}{$\begin{array}{c}\text { Estado } \\
\text { das } \\
\text { chaves }\end{array}$} \\
\hline S8 & S7 & S6 & S5 & S4 & S3 & S2 & S1 & & & $\mathrm{i}_{\mathrm{C} \uparrow>0}>0$ & $i_{\mathrm{C} i}<0$ & \\
\hline 1 & 0 & 1 & 0 & 1 & 0 & 1 & 0 & $-U$ & $-U$ & - & - & V1 \\
\hline 1 & 0 & 1 & 0 & 1 & 0 & 0 & 1 & $-U+U / 2$ & $-U / 2$ & Descarregado & Carregado & V2 \\
\hline 1 & 0 & 1 & 0 & 0 & 1 & 1 & 0 & $-U / 2$ & $-U / 2$ & Carregado & Descarregado & V3 \\
\hline 1 & 0 & 1 & 0 & 0 & 1 & 0 & 1 & 0 & 0 & - & - & V4 \\
\hline 0 & 1 & 0 & 1 & 1 & 0 & 1 & 0 & 0 & 0 & - & - & V5 \\
\hline 0 & 1 & 0 & 1 & 1 & 0 & 0 & 1 & $\mathrm{U} / 2$ & $U / 2$ & Descarregado & Carregado & V6 \\
\hline 0 & 1 & 0 & 1 & 0 & 1 & 1 & 0 & $\mathrm{U}-\mathrm{U} / 2$ & $\mathrm{U} / 2$ & Carregado & Descarregado & V7 \\
\hline 0 & 1 & 0 & 1 & 0 & 1 & 0 & 1 & $U$ & $U$ & - & - & V8 \\
\hline
\end{tabular}

Fonte: Dados da pesquisa do autor.

Na Figura 3.2 são mostrados cada estado de chaveamento conforme os níveis de tensão de fase.

Figura 3.2 - Estado de chaveamento de cada nível.

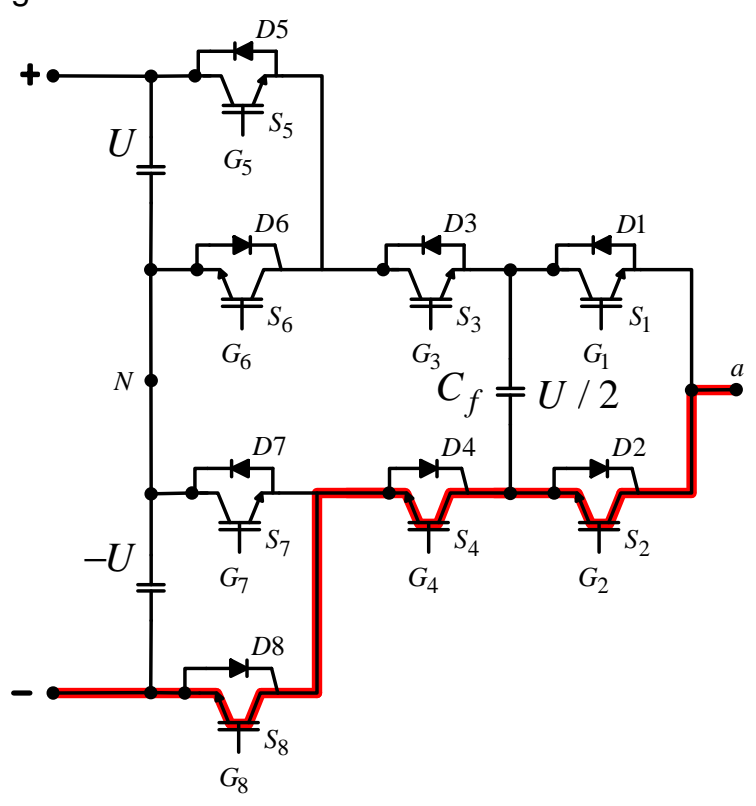

(a) $\mathrm{V} 1 \rightarrow(-\mathrm{U})$

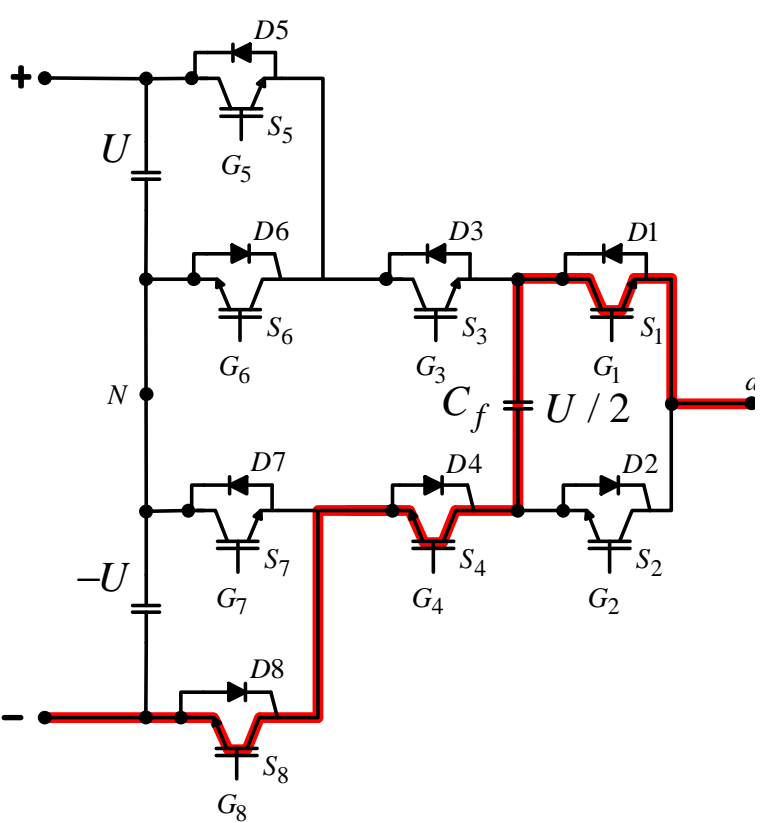

(b) $\mathrm{V} 2 \rightarrow(-\mathrm{U} / 2)$ 


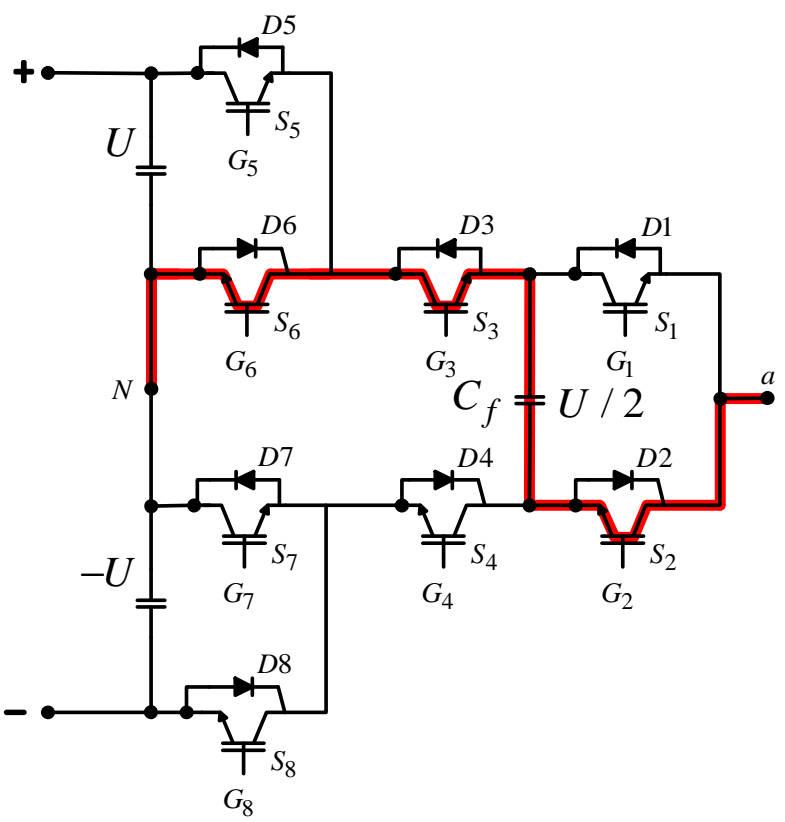

(c) $\mathrm{V} 3 \rightarrow(-\mathrm{U} / 2)$

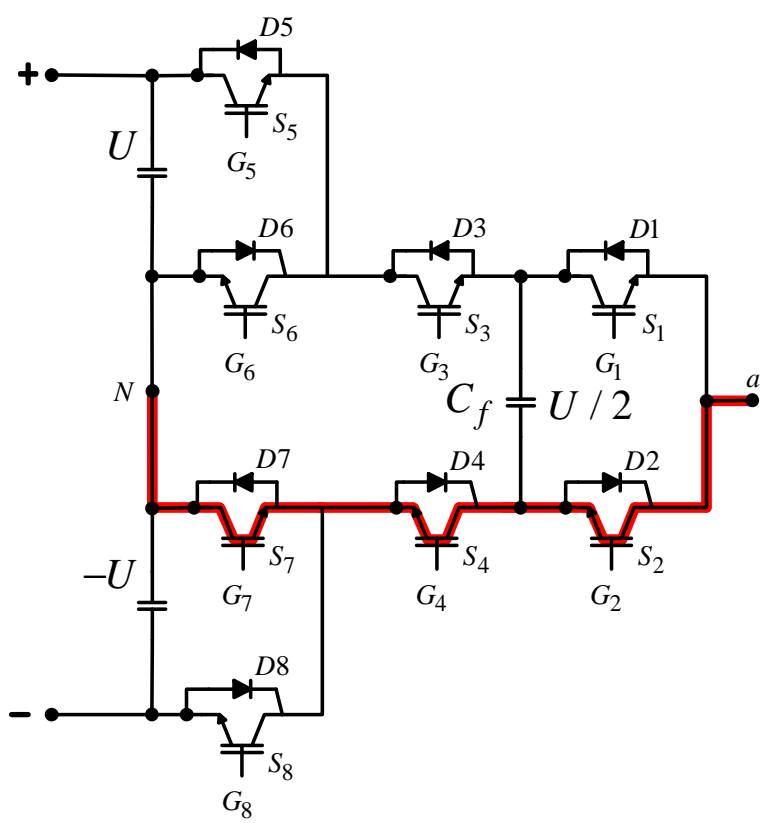

(e) V5 $\rightarrow(0)$

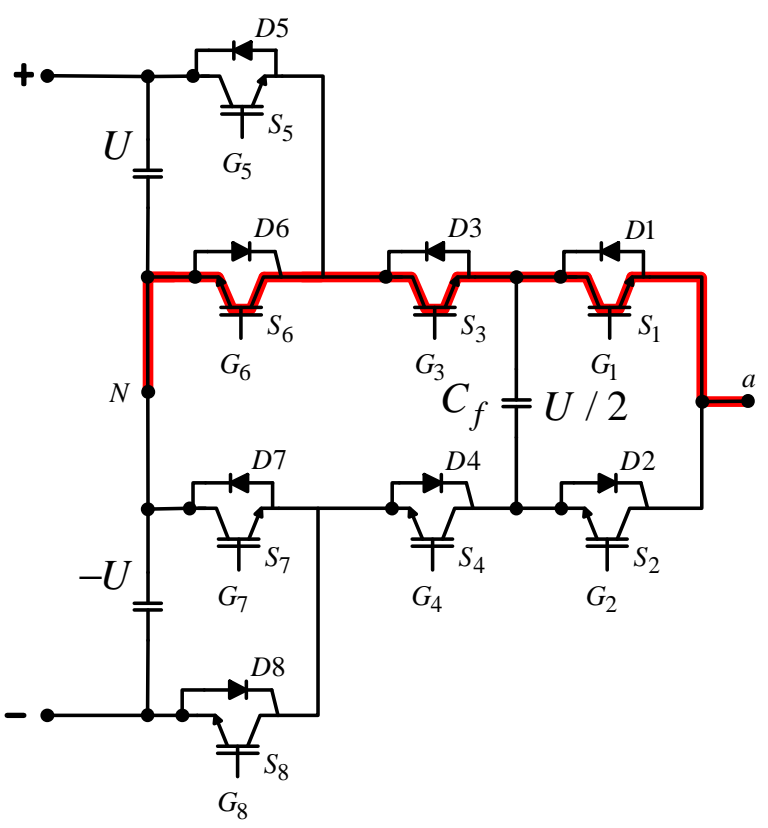

(d) V4 $\rightarrow$ (0)

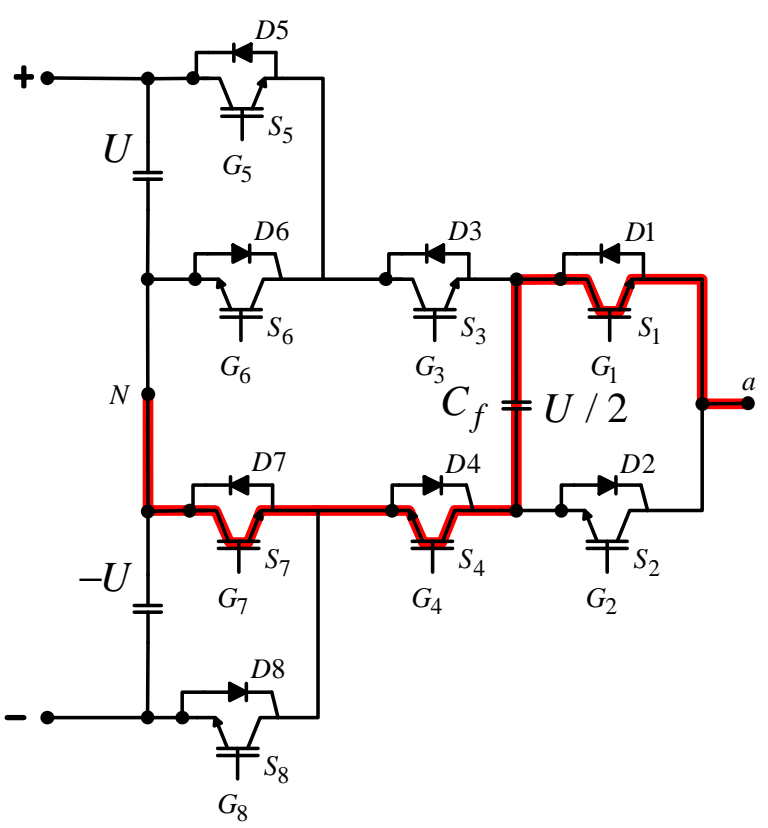

(f) $\mathrm{V} 6 \rightarrow(\mathrm{U} / 2)$ 


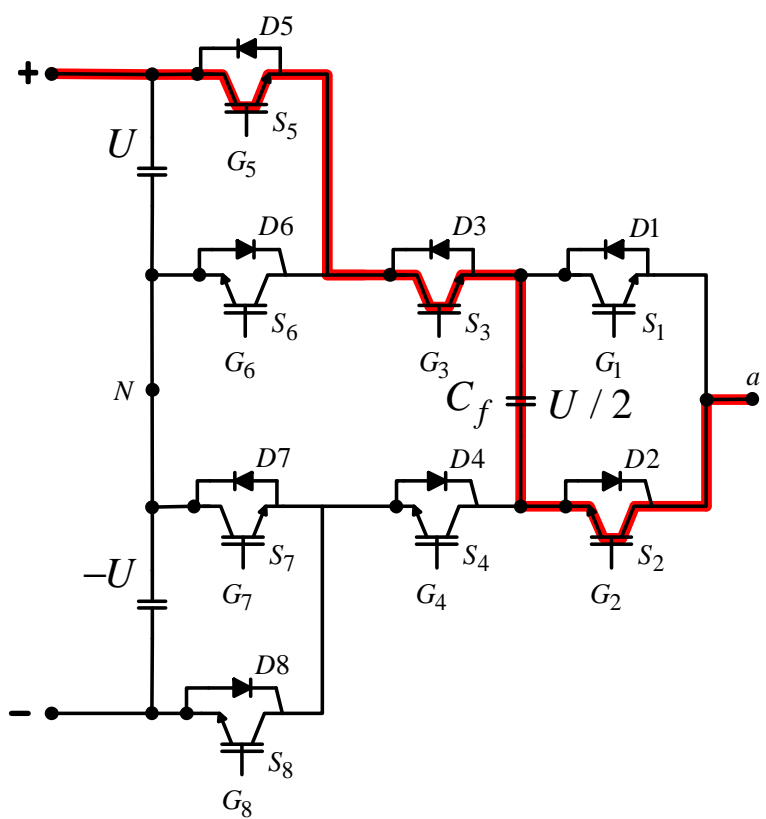

(g) $\mathrm{V} 7 \rightarrow(\mathrm{U} / 2)$

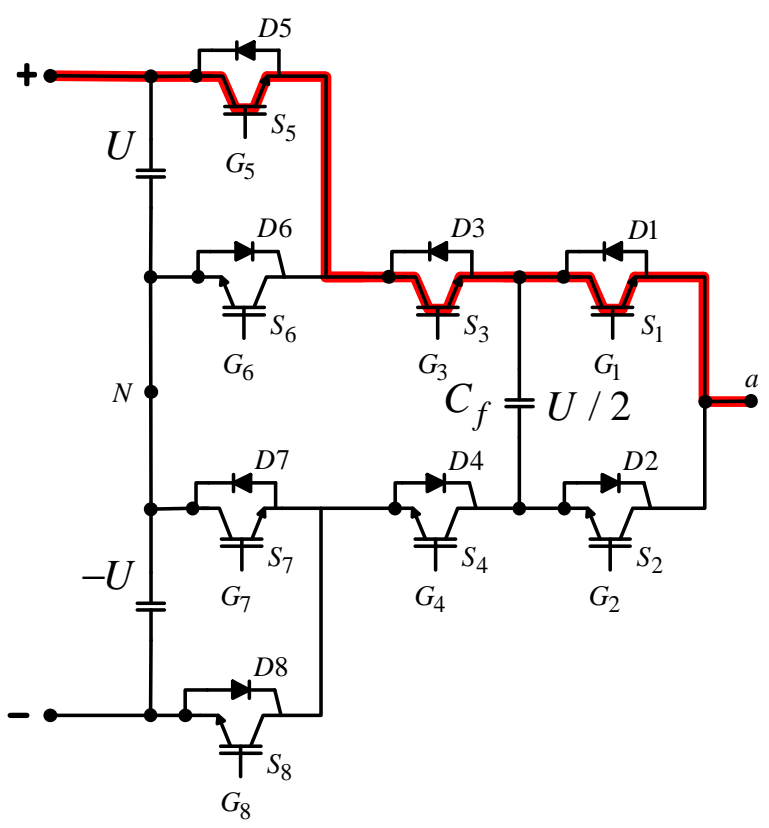

(h) $\mathrm{V} 8 \rightarrow(\mathrm{U})$

Fonte: Dados da pesquisa do autor.

Entretanto, as variações de tensão, $\Delta \mathrm{V}_{\mathrm{cfx}}$ nos estados de chaveamento $\mathrm{V} 2$ ou V3 (Vout $<0$ ) e V6 ou V7 (Vout $>0$ ), são utilizadas para carregar ou descarregar o

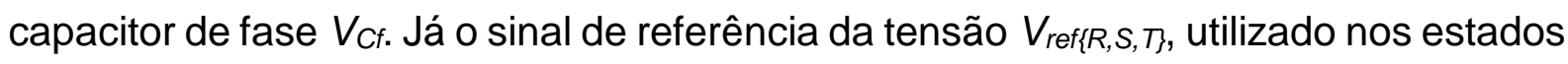
de chaveamento V4 ou V5 (valor zero) é selecionado a frequência de comutação dos semicondutores do módulo 1 na frequência fundamental, conforme mostra a Tabela 3.2.

A capacitância necessária para limitar a ondulação da tensão nos terminais do capacitor de fase para uma dada frequência de chaveamento pode ser calculada de acordo com

$$
C_{f}=\frac{I_{p k}}{\Delta V_{f}} \frac{1}{f_{c}},
$$

em que $I_{p k}$ é a corrente de pico, $\Delta v f$ é a tensão pico a pico nos terminais do capacitor de fase e $f_{c}$ é a frequência de chaveamento do conversor.

A energia armazenada no capacitor pode ser calculada conforme

$$
E_{\text {flutuante }}=\frac{1}{2} C_{f} \sum_{i=1}^{\frac{N-3}{2}}\left(\frac{i}{\frac{1}{2}(N-1)}\right)^{2},
$$


e normalizada por

$$
E_{\text {normalizado }}=\frac{E_{\text {flutuante }}}{\left(\frac{1}{2} C_{f} U^{2}\right)}=\sum_{i=1}^{\frac{N-3}{2}}\left(\frac{i}{\frac{1}{2}(N-1)}\right)^{2},
$$

em que $\mathrm{N}$ é o número de níveis da saída do conversor e $U$ é a metade da tensão do elo CC total, entre os pontos positivo e negativo.

Tabela 3.2 - Seleção dos estados de chaveamento - ANPC cinco níveis.

\begin{tabular}{c|c|c|c|c|c}
\hline $\begin{array}{c}\text { Tensão de } \\
\text { fase }\end{array}$ & $\begin{array}{c}\text { Número de } \\
\text { níveis }\end{array}$ & Sinal $\left(\Delta \mathrm{V}_{\mathrm{cfx}}\right)$ & Sinal $\left(\mathrm{i}_{\mathrm{x}}\right)$ & $\begin{array}{c}\text { Sinal }\left(\mathrm{V}_{\text {refx }}\right) \\
\mathrm{X} \in\{\mathrm{R}, \mathrm{S}, \mathrm{T}\}\end{array}$ & $\begin{array}{c}\text { Estado de } \\
\text { chaveamento }\end{array}$ \\
\hline$-\mathrm{U}$ & 0 & - & - & - & $\mathrm{V} 1$ \\
\hline$-\mathrm{U} / 2$ & 1 & -1 & -1 & - & $\mathrm{V} 2$ \\
\hline$-\mathrm{U} / 2$ & 1 & -1 & +1 & - & $\mathrm{V} 3$ \\
\hline$-\mathrm{U} / 2$ & 1 & +1 & -1 & - & $\mathrm{V} 3$ \\
\hline$-\mathrm{U} / 2$ & 1 & +1 & +1 & - & $\mathrm{V} 2$ \\
\hline 0 & 2 & - & - & -1 & $\mathrm{~V} 4$ \\
\hline 0 & 2 & - & - & +1 & $\mathrm{~V} 5$ \\
\hline $\mathrm{U} / 2$ & 3 & -1 & -1 & - & $\mathrm{V} 6$ \\
\hline $\mathrm{U} / 2$ & 3 & -1 & +1 & - & $\mathrm{V} 7$ \\
\hline $\mathrm{U} / 2$ & 3 & +1 & -1 & - & $\mathrm{V} 7$ \\
\hline $\mathrm{U} / 2$ & 3 & +1 & +1 & - & $\mathrm{V} 6$ \\
\hline $\mathrm{U}$ & 4 & - & - & - & $\mathrm{V} 8$ \\
\hline
\end{tabular}

Fonte: Dados da pesquisa do autor.

Na Figura 3.3 são demonstrados os sinais de saída do conversor ANPC cinco níveis de acordo com as tabelas 3.1 e 3.2.

Figura 3.3 - Sinal de saída do conversor ANPC cinco níveis.

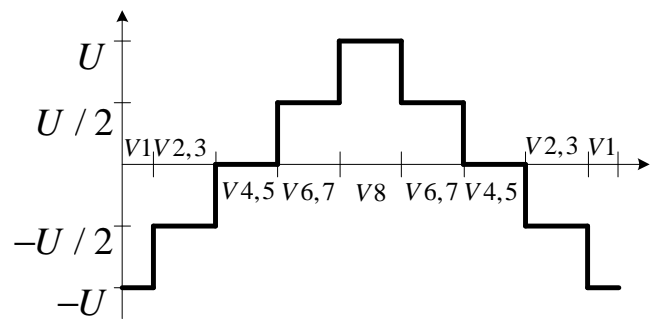

Fonte: Dados da pesquisa do autor. 
Estratégias de modulação aplicadas neste tipo de conversor multinível são basicamente as mesmas do conversor ANPC três níveis.

As pesquisas sobre os métodos de modulação têm como foco a busca de sequências de comutações ideais (Kim et al., 2008), a operação em baixo índice de modulação (Ben-Brahim et al., 2006), a adaptação às novas topologias (Loh et al., 2007; Teymour et al., 2015), e redução da tensão de modo comum (Videt et al., 2007).

\subsection{Modelo matemático do conversor multinível ANPC 5 níveis}

O modelo matemático representa as variações dos estados de chaveamento do conversor de cada nível apresentado nas tabelas 3.1 e 3.2, bem como na Figura 3.2. Já na Figura 3.4 estão representados somente os dispositivos semicondutores e elementos passivos que são chaveados em cada nível.

Figura 3.4 - Dispositivos semicondutores e elementos passivos que são chaveados em cada nível.

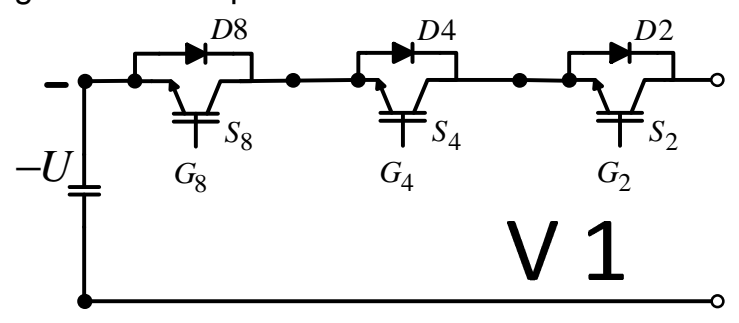

(a)

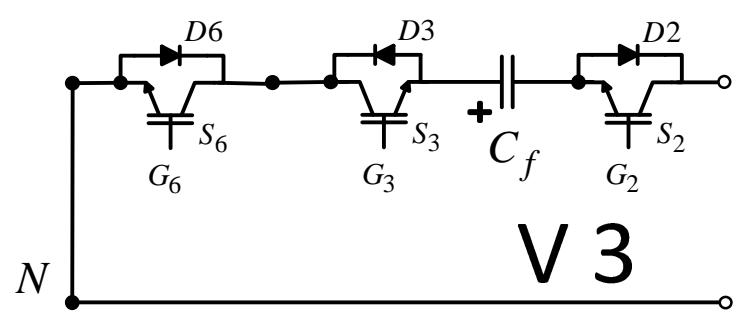

(c)

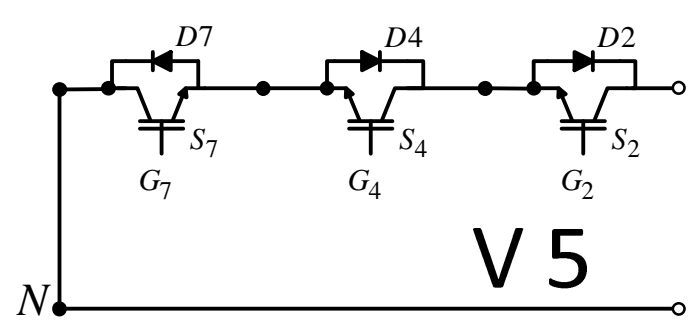

(e)

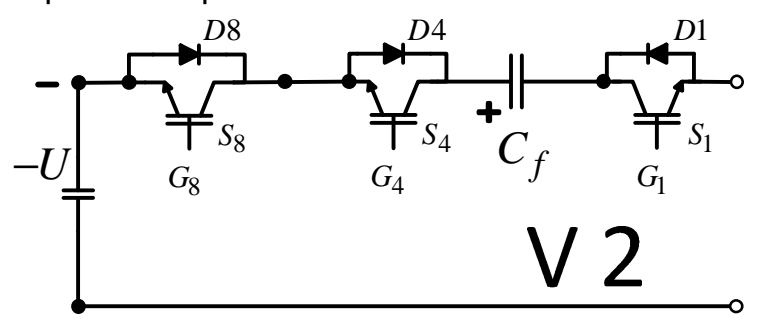

(b)

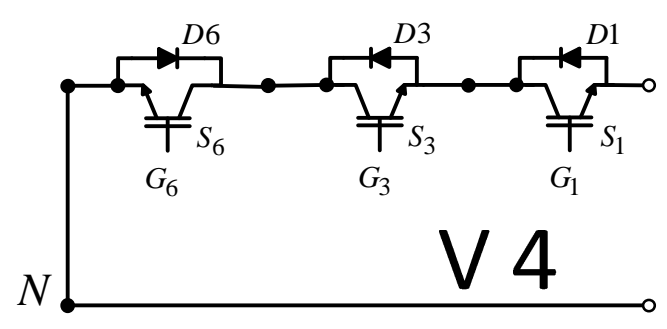

(d)

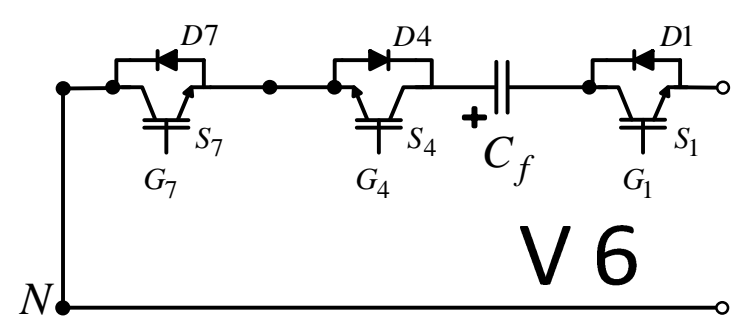

(f) 


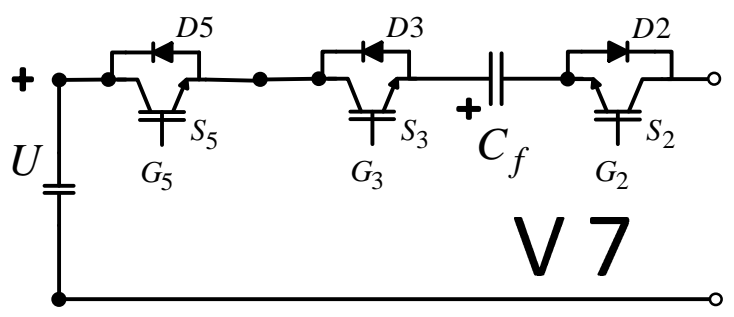

(g)

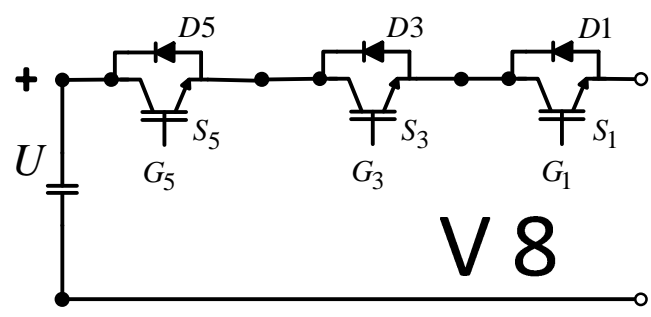

(h)

Fonte: Dados da pesquisa do autor.

$\log 0$

$$
\begin{aligned}
& R=\left(-U\left(1-d_{5}\right)\left(1-d_{3}\right)\left(1-d_{1}\right)\right)+\left(\left(-U+V_{C_{f}}\right)\left(1-d_{5}\right)\left(1-d_{3}\right) d_{1}\right)+ \\
& \left(-V_{C_{f}}\left(1-d_{5}\right) d_{3}\left(1-d_{1}\right)\right)+0+0+\left(V_{C_{f}} d_{5}\left(1-d_{3}\right) d_{1}\right)+ \\
& \left(\left(U-V_{C_{f}}\right) d_{5} d_{3}\left(1-d_{1}\right)\right)+\left(U d_{5} d_{3} d_{1}\right)
\end{aligned} .
$$

Realizando-se as operações matemáticas distributivas, tem-se

$$
R=\left(-U d_{3} d_{5}\right)+\left(U d_{5}\right)+\left(U d_{3}\right)+(-U)+\left(V_{C_{f}} d_{1}\right)+\left(-V_{C_{f}} d_{3}\right)+\left(U d_{3} d_{5}\right),
$$

Rearranjando

$$
R=\left(V_{C_{f}} d_{1}\right)+\left(\left(U-V_{C_{f}}\right) d_{3}\right)+\left(U d_{5}\right)+(-U),
$$

de modo que

$$
\frac{R}{d_{1}}=V_{C_{f}}=\frac{U}{2} ; \frac{R}{d_{3}}=\left(U-V_{C_{f}}\right)=\frac{U}{2} ; \frac{R}{d_{5}}=U
$$

Assim, é obtida a matriz que representa o modelo matemático do conversor multinível ANPC 5 níveis 


$$
R=V_{C_{f}}\left[\begin{array}{lll}
\frac{1}{2} & \frac{1}{2} & 1
\end{array}\right]\left[\begin{array}{l}
d_{1} \\
d_{3} \\
d_{5}
\end{array}\right]-V_{C_{f}} .
$$

De modo a confirmar o modelo matemático, foi realizada uma simulação computacional, em que foi comparado o modelo calculado com o circuito chaveado apresentado nas figuras 3.5 e 3.6, de modo a confirmar a equivalência entre ambos, validando, assim, o modelo matemático corresponde ao circuito chaveado.

Figura 3.5 - Forma de onda da saída do inversor chaveado e do modelo matemático - ANPC 5 níveis.

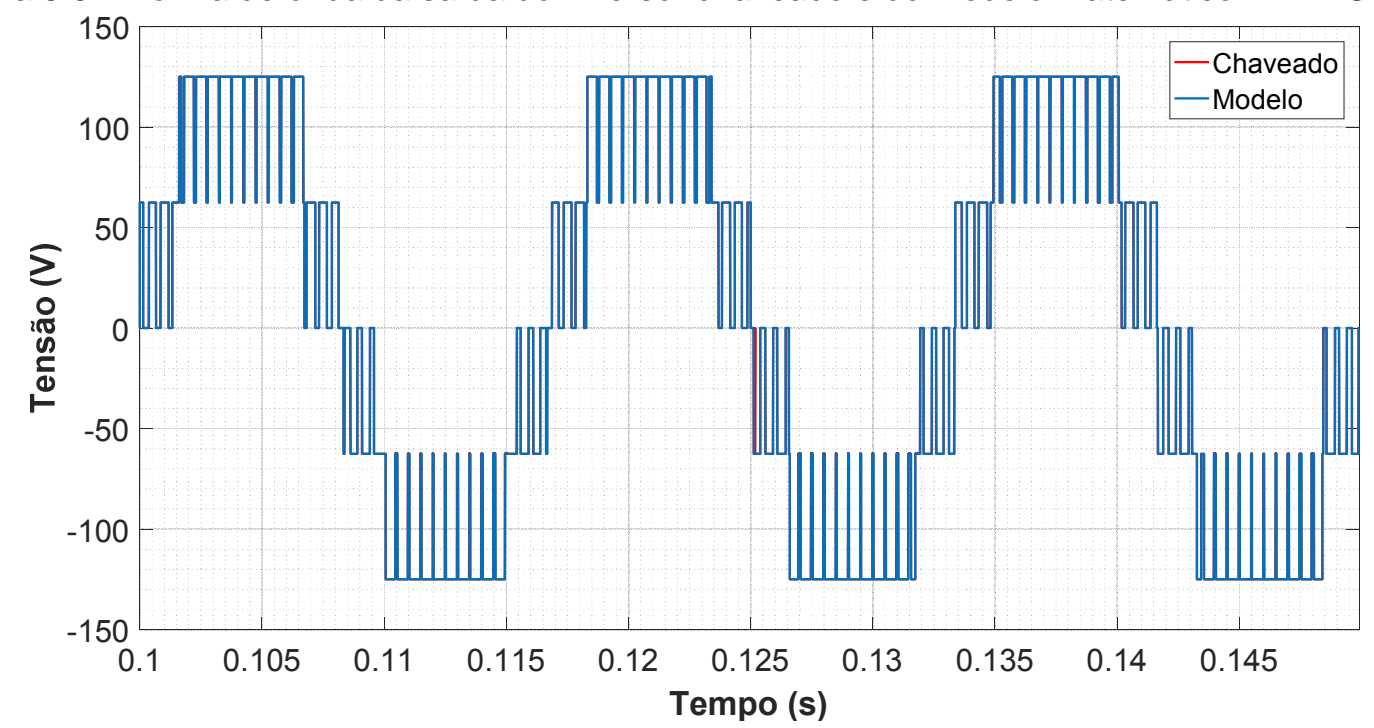

Fonte: Dados da pesquisa do autor.

Figura 3.6 - Forma de onda da saída do inversor. (a) chaveado; (b) modelo teórico.

(a)

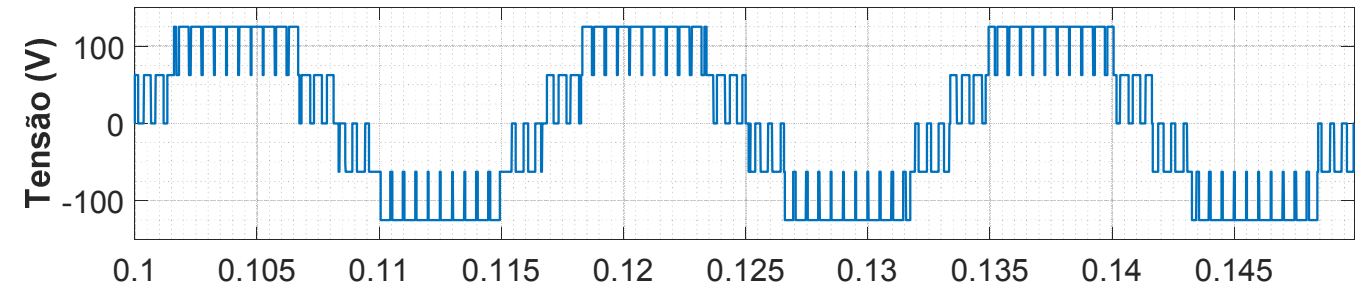

(b)

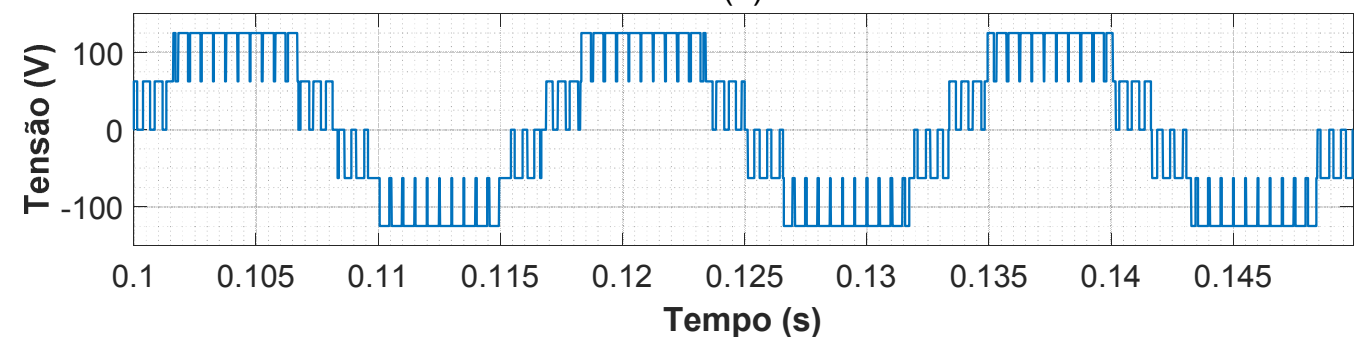

Fonte: Dados da pesquisa do autor. 


\subsection{Modulação do conversor multinível ANPC 5 níveis}

A modulação adotada nos conversores multiníveis é a Phase Disposition (PH) (Carrara et al., 1992), também conhecida como Level Shifted (McGrath et al., 2000), empregada por (Lau et al., 2004; Mei et al., 2014; Ghias et al., 2014; Jin; Yuan, 2016). Está técnica PWM utiliza vários sinais portadores triangulares em fase, comparando com apenas uma modulação senoidal. Assim, para o conversor multinível de 5 níveis, em que $N$ é igual a 5 , será preciso utilizar 4 portadoras, ou seja, $N-1$. Todas as portadoras têm a mesma frequência $\omega_{c}$ e o mesmo valor de pico a pico $A_{c}$. O sinal de modulação senoidal tem uma frequência $\omega_{m}$ e amplitude $A_{m}$. A cada instante, cada portadora é comparada com o sinal de modulação, conforme mostra a Figura 3.7(a), assim produzindo o sinal resultante na saída do conversor multinível. Já na Figura 3.7(b), são apresentados os sinais PWM dos pulsos referentes às triangulares comparados com a modulação senoidal.

Os parâmetros do processo de modulação são:

- O número de níveis do inversor,

$$
N^{\prime}=(N-1) / 2 .
$$

- A relação entre a frequência da portadora com a frequência da modulação, é

$$
P=\omega_{c} / \omega_{m} .
$$

- O índice de modulação,

$$
M=A_{m} /\left(N^{\prime} A_{c}\right) .
$$

- O ângulo $\phi$ de deslocamento entre a referência senoidal e o valor positivo do sinal da portadora. 
Figura 3.7 - (a) Phase Disposition (PH); (b) Padrão dos pulsos PWM. Parâmetros: $M=1, P=33,33$ e $\phi=0$.

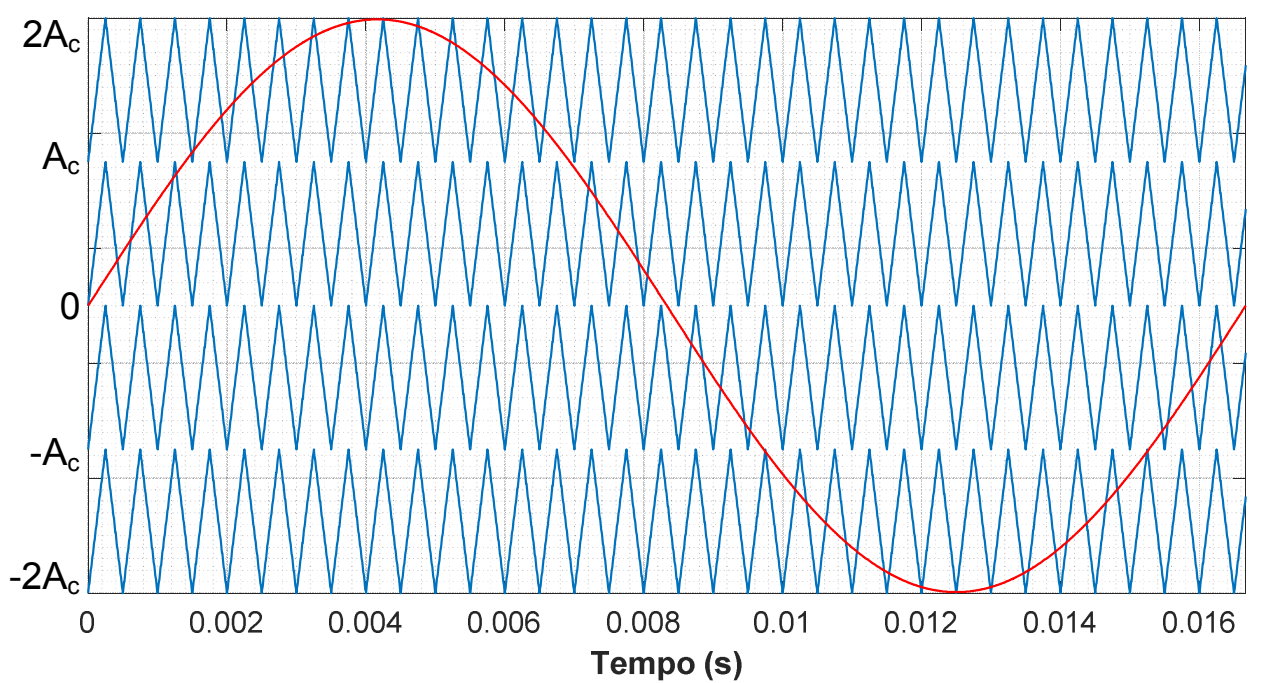

(a)

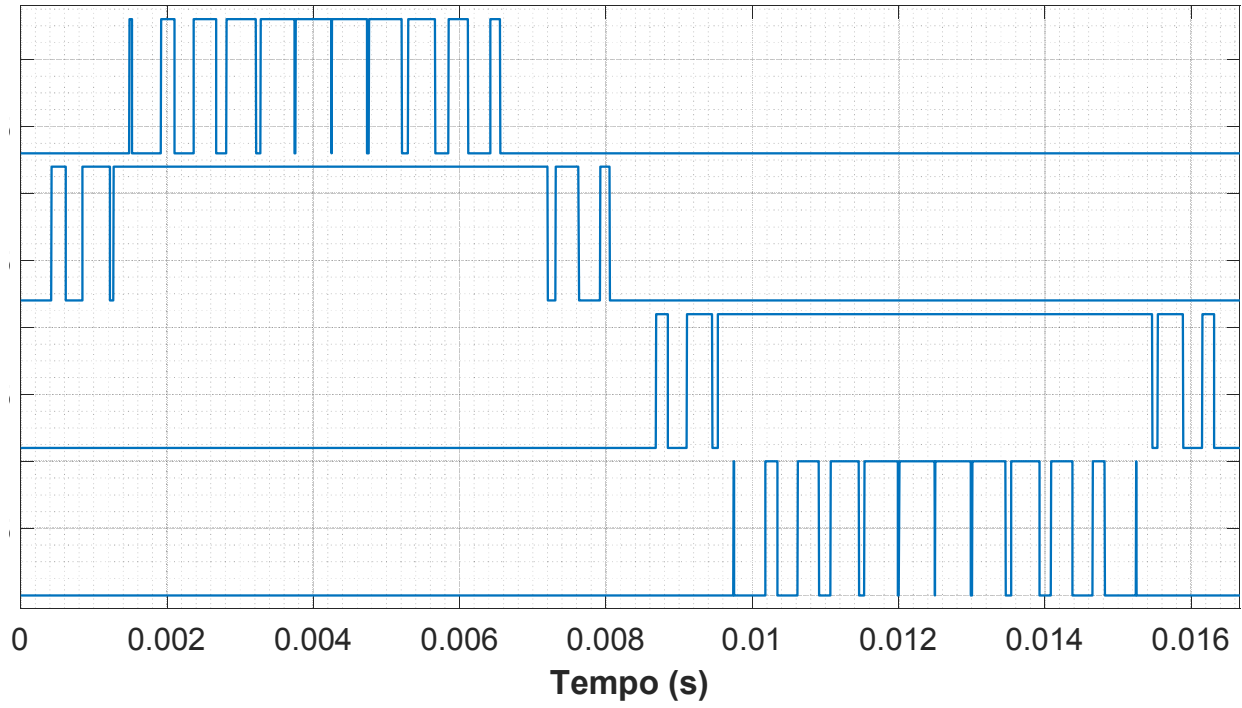

(b)

Fonte: Dados da pesquisa do autor.

Por meio do método apresentado por Bennett (Bowes, 1975; Black, 1953), é possível calcular os componentes espectrais da tensão de saída do conversor sob qualquer condição de operação. Além disso, uma vez que o resultado é uma expressão da série dupla de Fourier na qual se adapta a processos de modulação síncrona e assíncrona. Para encontrar a expressão temporal do sinal PWM, deve-se ter as seguintes relações:

$$
\begin{aligned}
& x=\omega_{m} t \\
& y=\omega_{c} t
\end{aligned}
$$


Neste caso, tendo um conjunto de $N-1$ portadoras, aplica-se essa relação para cada sinal portador, assim, subdividindo o processo multinível em $\mathrm{N}-1$ processos bipolares. Cada processo bipolar tem uma função $F_{i}(x, y)$, que assumem valores entre 0 e $U$, ou 0 e $-U$. Assim, soma-se todas as funções $F_{i}(x, y)$ de cada portadora para se obter o resultado final. A função $F_{i}(x, y)$ é periódica, e varia entre $0 \leq x \leq 2 \pi$ e $-\pi \leq y \leq \pi$.

Para se definir $N^{\prime \prime}$, precisa-se da influência dos parâmetros $N^{\prime}$ e $M$, bem como o número de níveis positivos exigidos pelo processo de modulação, assim

$$
\begin{aligned}
& M=1 / N^{\prime} \\
& N^{\prime \prime}=\left[M N^{\prime}\right]+1
\end{aligned} .
$$

$F_{i}(x, y)$ pode ser mostrado como uma série dupla de Fourier, conforme

$$
F(x, y)=\frac{1}{2} A_{0,0}+\sum_{n=1}^{+\infty}\left[A_{n, 0} \cos (n x)+B_{n, 0} \operatorname{sen}(n x)\right]+\sum_{m=1}^{+\infty} \sum_{n=-\infty}^{+\infty}\left[\begin{array}{l}
A_{n, m} \cos (n x+m y)+ \\
B_{n, m} \operatorname{sen}(n x+m y)
\end{array}\right],
$$

em que

$$
F_{n, m}=A_{n, m}+j B_{n, m}=\frac{1}{2 \pi^{2}} \int_{x^{*}}^{x^{*}+2 \pi y^{*}} \int_{y^{*}}^{* 2 \pi} F(x, y) e^{j(n x+m y)} d x d y .
$$

A área de integração é $0 \leq x \leq 2 \pi$ e $+\pi \leq y \leq \pi$, que fornece $x^{*}=0$ e $y^{*}=-\pi$. Para calcular os coeficientes das série de Fourier, divide-se a área de integração em um número de partes, sendo $\left(\mathrm{x}_{\mathrm{i},-}-\pi\right),\left(\mathrm{x}_{\mathrm{i}}, \pi\right),\left(\mathrm{x}_{\mathrm{i}+1}, \pi\right)$ e $\left(\mathrm{x}_{\mathrm{i}+1},-\pi\right)$.

A função $F$ não tem propriedades de simetria, portanto, os termos seno e cosseno estão presentes na série de Fourier como

$$
B=i \pi ; i \in Z ; Q=M N^{\prime} \pi
$$

Assim, o resultado é mostrado em (3.19 a 3.21). 


$$
\begin{aligned}
& v=\frac{V}{\pi}\left\{M\left[2 x_{N^{\prime}+1}-\operatorname{sen}\left(2 x_{N^{\prime}+1}\right)\right]+4 \cos \left(x_{N^{\prime}+1}\right)\right\} \\
& \operatorname{sen}\left(w_{m} t+\phi\right)+\frac{V}{\pi} \int_{n=3}^{+\infty}\left[1-(-1)^{n}\right] \\
& \left\{\begin{array}{l}
M\left[\frac{\operatorname{sen}\left[(n-1) x_{N^{\prime}+1}\right]}{n-1}-\frac{\operatorname{sen}\left[(n+1) x_{N^{\prime}+1}\right]}{n+1}\right] \\
+\frac{2}{n} \cos \left(n x_{N^{\prime}+1}\right)
\end{array}\right\} \operatorname{sen}\left(n \omega_{m} t+n \phi\right)+\frac{V}{\pi^{2} N^{\prime}} \sum_{m=1}^{+\infty} \\
& \frac{1}{m} \sum_{n=-\infty}^{+\infty}\left\{C_{m, n} \cos \left(m \omega_{c} t+n \omega_{m} t+n \phi\right)+S_{m, n} \operatorname{sen}\left(m \omega_{c} t+n \omega_{m} t+n \phi\right)\right\}
\end{aligned}
$$

em que

$$
\begin{gathered}
C_{m, n}=\left[1+(-1)^{n}\right]\left[1-(-1)^{m}\right] \sum_{h} J_{h}\left(m M N^{\prime} \pi\right) \frac{1}{n+h} \\
\sum_{k=1}^{N^{\prime}} \cos [m(k-1) \pi]\left\{\cos \left[(n+h) x_{k+1}\right]-\cos \left[(n+h) x_{k}\right]\right\} \\
S_{m, n}=\left[1-(-1)^{n}\right]\left[1+(-1)^{m}\right] \\
\left\{\begin{array}{l}
J_{n}\left(m M N^{\prime} \pi\right)\left\{\sum_{k=1}^{N^{\prime}} \cos [m(k-1) \pi]\left(x_{k+1}-x_{k}\right)\right\}-\sum_{h \neq-n} J_{h}\left(m M N^{\prime} \pi\right) \frac{1}{n+h} \\
\sum_{k=1}^{N^{\prime}} \cos [m(k-1) \pi]\left\{\operatorname{sen}\left[(n+h) x_{k+1}\right]-\operatorname{sen}\left[(n+h) x_{k}\right]\right\}
\end{array}\right.
\end{gathered}
$$

Esta técnica de modulação multinível produz sinais modulados consistindo de termos que fornecem a amplitude do valor fundamental, que é diretamente proporcional ao índice de modulação $M$ até $M \leq 1$. Um termo para $m=0$ dando a amplitude dos harmônicos em múltiplos da frequência de modulação. Um outro termo para $n=0$, sendo a amplitude dos harmônicos na frequência da portadora e múltiplos. Neste caso, não existem harmônicos em múltiplos pares da frequência da portadora do sinal. O mesmo termo para $m$ fixo e $n$ variável fornece a amplitude dos harmônicos nas bandas laterais centrado em torno de cada múltiplo da frequência da portadora. Apenas componentes de ordem par existem nas bandas laterais centradas em torno de harmônicos ímpares da frequência da portadora e vice-versa. As amplitudes desses componentes reduzem a modulação quando $M$ aumenta. 
De forma a implementar a modulação no DSP ponto flutuante, em que, utilizase somente uma triangular, foi realizada uma abordagem que pode ser usada para desenvolver o espectro harmônico de uma referência senoidal modulada por uma portadora triangular. Para uma portadora triangular, a forma de onda de referência tem um ciclo de trabalho $d=(1+M \cos y) / 2$, mais uma vez, é inicialmente assumido como constante dentro do intervalo de portadora (Holmes; Lipo, 2003). Para este caso, a série de Fourier correspondente à forma de onda comutada resultante tornase

$$
v_{a n}=\frac{a_{0}}{2}+\sum_{m=1}^{\infty}\left(a_{m} \cos m x+b_{m} \operatorname{senm} x\right)
$$

em que,

$$
\begin{gathered}
a_{m}=\frac{1}{\pi} \int_{-\pi}^{\pi} v_{a n}(t) \cos m x d x=\frac{2 V_{c c}}{\pi} \int_{-\frac{\pi}{2}(1+M \cos y)}^{\frac{\pi}{2}(1+M \cos y)} \cos m x d x \\
=\frac{4 V_{c c}}{m \pi}\left[\operatorname{sen}\left(m \frac{\pi}{2}(1+M \cos y)\right)\right] \rightarrow m \neq 0
\end{gathered}
$$

e,

$$
b_{m}=\frac{1}{\pi} \int_{-\pi}^{\pi} v_{a n}(t) \operatorname{senm} x d x=\frac{2 V_{c c}}{\pi} \int_{-\frac{\pi}{2}(1+M \cos y)}^{\frac{\pi}{2}(1+M \cos y)} \operatorname{senm} x d x=0
$$

De acordo com as equações (3.22), (3.23), (3.24) e (3.25), tem-se

$$
\begin{gathered}
V_{a n}=V_{c c}(1+M \cos y)+\frac{4 V_{c c}}{\pi} \sum_{m=1}^{\infty} \frac{1}{m} \operatorname{sen} m \frac{\pi}{2} J_{0}\left(m \frac{\pi}{2} M\right) \cos m x \\
+\frac{8 V_{c c}}{\pi} \sum_{m=1}^{\infty} \frac{1}{m} \sum_{n=1}^{\infty}\left[\begin{array}{c}
\operatorname{sen} m \frac{\pi}{2} \cos n \frac{\pi}{2} J_{n}\left(m \frac{\pi}{2} M\right) \\
\left.+\cos m \frac{\pi}{2} \operatorname{sen} n \frac{\pi}{2} J_{n}\left(m \frac{\pi}{2} M\right)\right] \cos n y \cos m x
\end{array} .\right.
\end{gathered}
$$

Rearranjando a equação (3.26), tem-se 


$$
\begin{aligned}
& V_{a n}=V_{c c}+V_{c c} M \cos y+\frac{4 V_{c c}}{\pi} \sum_{m=1}^{\infty} \frac{1}{m} J_{0}\left(m \frac{\pi}{2} M\right) \operatorname{sen} m \frac{\pi}{2} \\
& +\frac{4 V_{c c}}{\pi} \sum_{m=1}^{\infty} \sum_{n=-\infty}^{\infty} \frac{1}{m} J_{n}\left(m \frac{\pi}{2} M\right) \operatorname{sen}\left([m+n] \frac{\pi}{2}\right) \cos (m x+n y)
\end{aligned} .
$$

De forma a implementar a modulação PH descrita acima no conversor ANPC 5 níveis conforme as tabelas 3.1 e 3.2, desenvolveu-se o fluxograma apresentado na Figura 3.8, bem como a comparação entre a triangular e a referência na Figura 3.9. Inicialmente, fazem-se as leituras do erro de tensão do capacitor flutuante $V_{C f e}$, da corrente de saída do conversor $I_{C f}$ e da referência, que pode ser a saída da ação de controle, tanto de um inversor de tensão $V_{\text {ref }}$ como de um inversor de corrente $I_{\text {ref. }}$. Após as leituras preestabelecidas, fazem-se as verificações de modo a saber se os valores de $V_{C f e}$ e $I_{C f}$ são maiores ou menores que 0 , e armazenam-se esses dados.

Já na verificação de $V_{\text {ref }}$ ou $I_{\text {ref, }}$ determina-se os valores são maiores ou menores que $\pi$. Caso o valor seja maior que $\pi$, ou seja, esteja no semiciclo negativo, aciona-se S5 (pulso baixo), e verifica-se se o sinal está no nível $0\left(\phi_{3}<\omega t<\phi_{4}\right)$ ou no nível $1\left(\pi<\omega t \leq \phi_{3}\right.$ ou $\left.\phi_{4}<\omega t \leq 2 \pi\right)$. Se o valor for menor que $\pi$, ou seja, esteja no semiciclo positivo, aciona-se S5 (pulso alto), e verifica-se se o sinal está no nível $2\left(0<\omega t \leq \phi_{1}\right.$ ou $\left.\phi_{2}<\omega t \leq \pi\right)$ ou no nível $3\left(\phi_{1}<\omega t<\phi_{2}\right)$, conforme Figura 3.8 e 3.10 .

Após as verificações das variáveis de tensão e de corrente enquadradas entre 0 e 1 , e definindo que seus níveis estão entre 0 e 3, carregam-se essas variáveis e realiza-se uma comparação com as tabelas 3.1 e 3.2, em que são avaliados os semiciclos e os estados de chaveamento. Após isso, acionam-se S1 e S3, conforme ilustrado na Figura 3.10. Essas operações são realizadas a cada interrupção de clock do processador de ponto flutuante. 
Figura 3.8 - Fluxograma da lógica de implementação da modulação multinível ANPC 5 níveis.

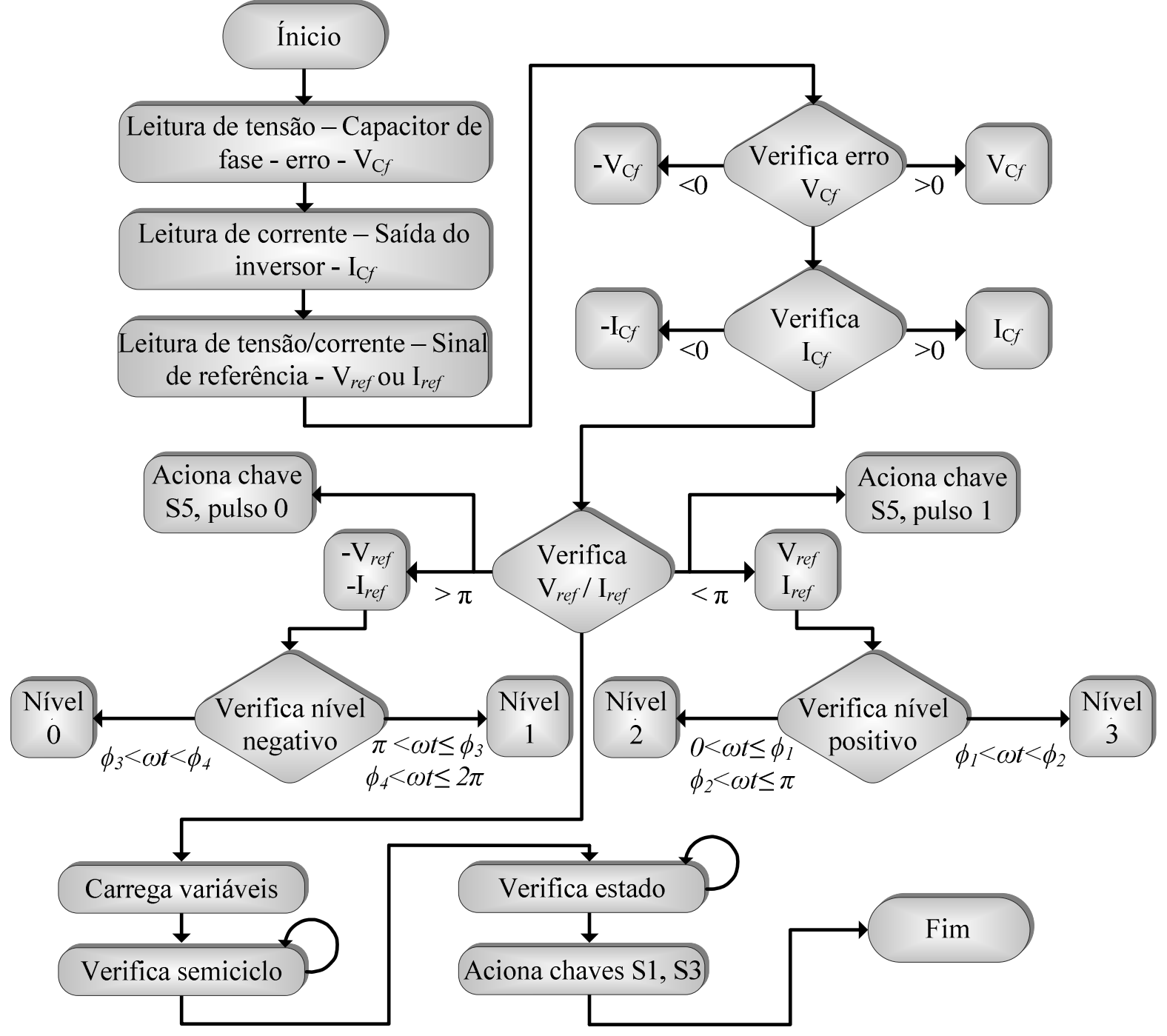

Fonte: Dados da pesquisa do autor.

Figura 3.9 - Phase Disposition - PH, níveis entre 0 e 3.

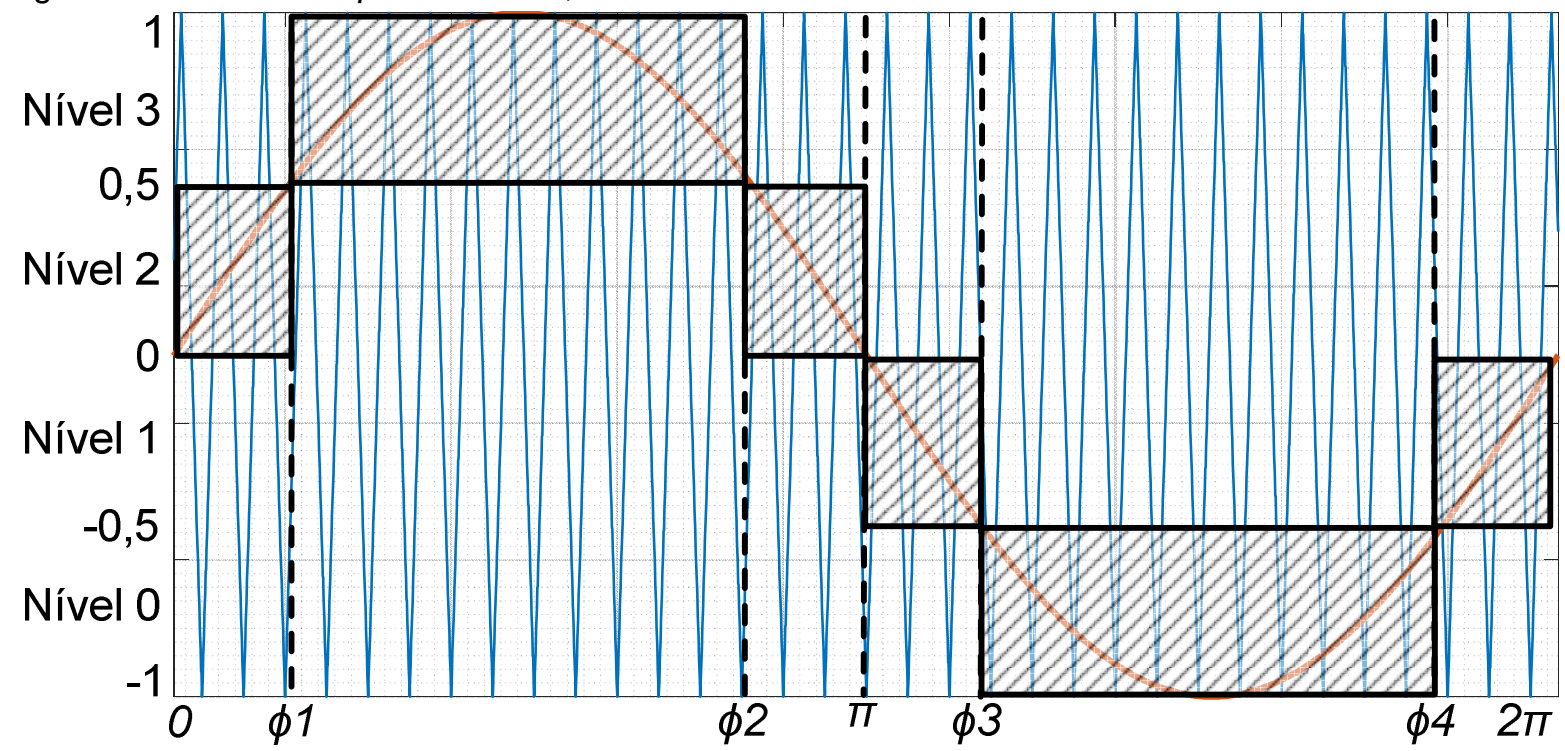

Fonte: Dados da pesquisa do autor. 
Figura 3.10 - Pulsos S1, S3 e S5 - Phase Disposition - PH.

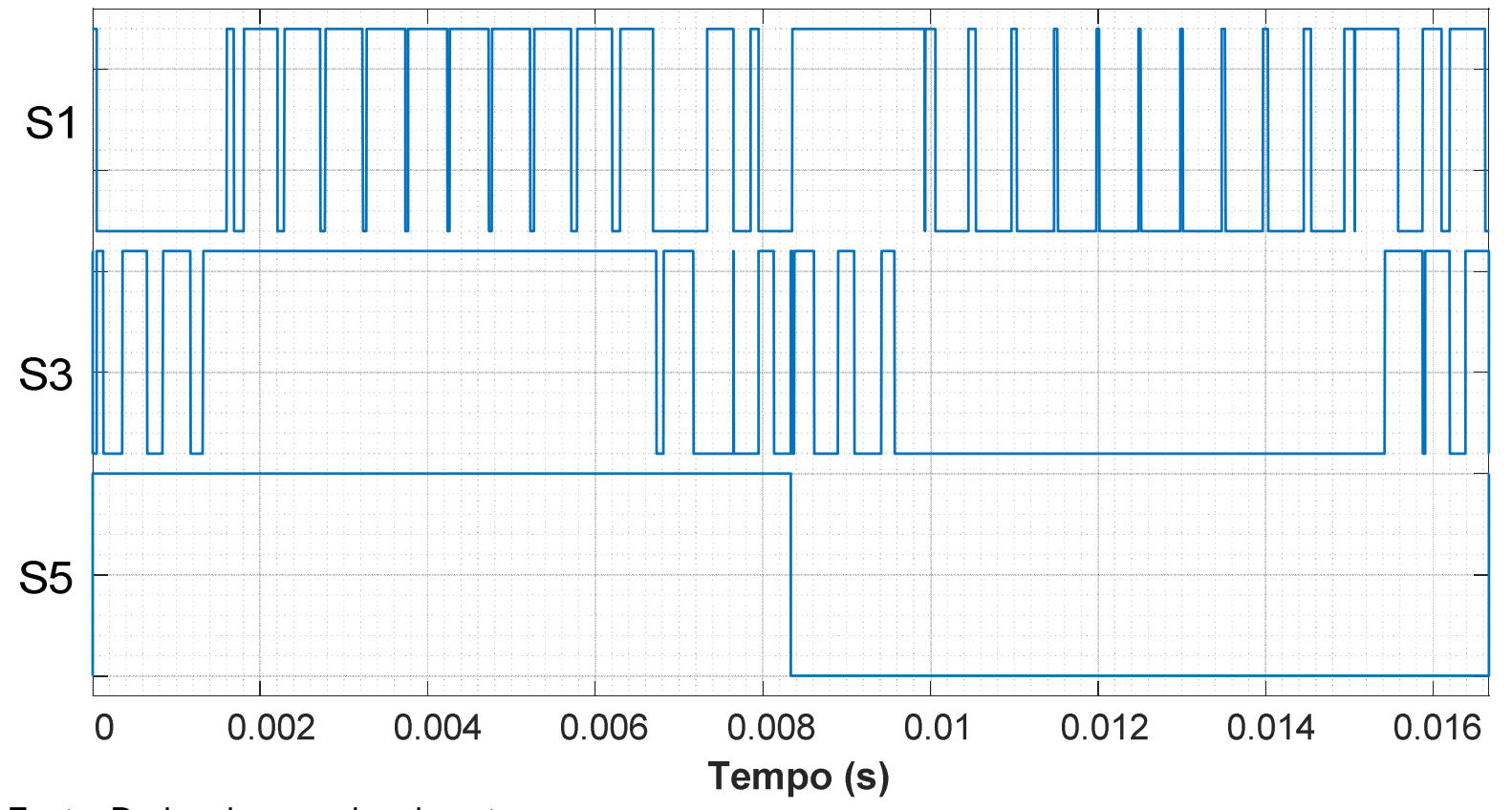

Fonte: Dados da pesquisa do autor.

\subsection{Considerações finais do capítulo}

Este capítulo apresentou o princípio de funcionamento do conversor ANPC 5 níveis, modelo matemático teórico e chaveado, modulação $\mathrm{PH}$ e fluxograma de implementação da modulação em microcontrolador de ponto flutuante. 


\section{Compensação Cooperativa baseada na Teoria da Potência Conservativa}

\subsection{Introdução}

A proposta de compensação cooperativa baseada na Teoria de Potência Conservativa (TPC) foi apresentada inicialmente em 2004 (Tenti; Mattavelli, 2004), considerando apenas filtros ativos e, desde então, vem sendo aprimorada de forma a agregar as outras tecnologias de compensadores e abranger vários distúrbios de Qualidade de Energia (Tedeschi et al., 2007, 2008; Tedeschi, 2009; Tedeschi; Tenti; Mattavelli, 2008; Tenti et al., 2009; Paredes; Costabeber, 2010; Paredes, 2011).

Os FAP, quando são submetidos a técnicas adequadas de controle cooperativo, podem desempenhar funções de otimização global, a fim de oferecer suporte à tensão, balanceamento das correntes da carga, minimização das distorções harmônicas e limitação das perdas na distribuição (Paredes, 2011).

Assim, visa-se selecionar e distribuir os distúrbios de qualidade de energia entre os compensadores, baseando-se na decomposição da corrente definida na TPC (Tenti; Mattavelli, 2004; Tenti et al., 2010) aplicados a um condicionador unificado de qualidade de energia, sendo que não há aplicações dessa forma apresentadas na literatura.

A TPC define, para algumas grandezas elétricas, uma propriedade chamada de conservativa (Tenti; Mattavelli, 2004; Tenti et al., 2010). Uma grandeza elétrica é dita conservativa se é calculada a partir do produto escalar entre tensões e correntes, de um circuito com $N$ fases, cujas tensões e correntes, assim como suas derivadas e integrais imparciais, satisfazem as leis de tensões e correntes de Kirchhoff e o teorema de Tellegen (Penfield et al., 1970), que representam a propriedade fundamental de conservação de energia, e os produtos das tensões das $N$ fases pelas correntes das $N$ fases, quando somados, resultam zero.

\subsection{Potência instantânea e energia reativa instantânea}

Enquadram-se na definição da propriedade conservativa as grandezas potência instantânea " $p$ " e energia reativa instantânea "w" (Paredes, 2011). Estas 
grandezas podem ser definidas como funções do tempo calculadas pelo produto escalar definido como a potência instantânea coletiva,

$$
p=\underline{v}^{*} \underline{i}=\sum_{\mu=1}^{m} v_{\mu} i_{\mu}
$$

e a potência instantânea de fase,

$$
p_{\mu}=v_{\mu} i_{\mu}
$$

de modo que a energia instantânea coletiva é dada como

$$
w_{r}=\underline{\hat{v}}^{*} \underline{i}=\sum_{\mu=1}^{m} \hat{v}_{\mu} i_{\mu},
$$

e a energia instantânea de fase definida como

$$
w_{r \mu}=\hat{v}_{\mu} i_{\mu},
$$

Em que ve i são vetores que contêm tensões e correntes em um sistema polifásico, bem como $\hat{v}$ é o vetor contendo as integrais imparciais das tensões de cada fase. Destaca-se que a potência instantânea e energia reativa instantânea são conservativas e suas respectivas unidades são: $[\mathrm{W}]$ watts e [J] joules.

A potência ativa calculada a partir da equação (4.2) equivale à definição de potência ativa convencionalmente utilizada. Porém, para caracterizar o fenômeno físico de defasagem entre tensão e corrente, os autores da TPC (Paredes, 2011) preferiram utilizar a energia relacionada a este fenômeno ao invés da potência, pois a energia reativa calculada através da equação (4.3) é um valor independente da frequência, o que torna essa grandeza interessante no tratamento de grandezas não senoidais e com componentes harmônicas presentes. 


\subsection{Potência ativa e Energia reativa}

A potência ativa coletiva é definida como

$$
P=\langle\underline{v}, \underline{i}\rangle=\frac{1}{T} \int_{0}^{T} \underline{v}^{*} \underline{i} d t=\frac{1}{T} \sum_{\mu=1}^{m} \int_{0}^{T} v_{\mu} i_{\mu}(t) d t=\sum_{\mu=1}^{m} P_{\mu}
$$

e a potência ativa por fase é

$$
P_{\mu}=\left\langle v_{\mu}, i_{\mu}\right\rangle=\frac{1}{T} \int_{0}^{T} v_{\mu} i_{\mu} d t
$$

que representam os valores médios da potência instantânea em condições senoidais e equilibradas. A potência ativa representa o fluxo permanente de energia, que não depende da referência de tensão (Blondel, 1893), bem como também é uma quantidade conservativa.

A energia reativa coletiva é

$$
W_{r}=\langle\underline{\hat{v}}, \underline{i}\rangle=\frac{1}{T} \int_{0}^{T} \underline{\hat{v}}^{*} \underline{i} d t=\frac{1}{T} \sum_{\mu=1}^{m} \int_{0}^{T} \hat{v}_{\mu} i_{\mu} d t=\sum_{\mu=1}^{m} W_{r \mu},
$$

e a energia reativa de fase é

$$
W_{r \mu}=\left\langle\hat{v}_{\mu}, i_{\mu}\right\rangle=\frac{1}{T} \int_{0}^{T} \hat{v}_{\mu} i_{\mu} d t
$$

em que $W_{\mu}$ e $W_{r \mu}$ representam o valor médio dos termos instantâneos da energia reativa, e são grandezas conservativas. Em geral, não é válido para a potência reativa (Q).

As quantidades de fase sob condições senoidais são dadas por

$$
\omega W_{r}=V I \sin \varphi=Q
$$


em que $Q$ é a potência reativa usual, que, sob apenas essa condição senoidal, é conservativa.

Independente da forma de onda das tensões e correntes, os termos potência instantânea $p$, potência ativa $P$, energia reativa instantânea $w_{r}$ e energia reativa $W_{r}$ são quantidades conservativas.

\subsection{Definição de termos de corrente e potência em circuitos monofásicos}

A fim de realizar a decomposição da corrente, tendo um significado específico em todos os termos, utilizam-se como base as definições anteriores de potência ativa e de energia reativa. A operação periódica (senoidal ou não) pode ser decomposta em três componentes: ativa, reativa e residual (nula).

\subsubsection{Corrente ativa}

A corrente ativa $i_{a}$ é definida de acordo com a norma mínima necessária para transportar potência ativa $P$,

$$
i_{a}=\frac{\langle v, i\rangle}{\|v\|^{2}} v=\frac{P}{V^{2}} v=G_{e} v
$$

em que $G_{e}$ é a condutância equivalente e $V$ é o valor eficaz da tensão. Dessa forma, têm-se

$$
\begin{gathered}
P_{a}=\left\langle v, i_{a}\right\rangle=G_{e}\langle v, v\rangle=G_{e}\|v\|^{2}=G_{e} V^{2}=P, \\
\left\|i_{a}\right\|=I_{a}=\frac{P}{V}, \\
W_{r a}=\left\langle\hat{v}, i_{a}\right\rangle=G_{e}\langle\hat{v}, v\rangle=0 .
\end{gathered}
$$


Na equação (4.12), tem-se o valor eficaz da corrente ativa. Assim, a corrente ativa transporta toda a potência ativa (total) e zero de energia reativa. A equação (4.10) coincide com a corrente ativa definida por (Fryze, 1932).

\subsubsection{Corrente reativa}

A corrente reativa $i_{r}$ é definida de acordo com a norma mínima necessária para transportar energia reativa $W_{r}$,

$$
i_{r}=\frac{\langle\hat{v}, i\rangle}{\|\hat{v}\|^{2}} \hat{v}=\frac{W_{r}}{\hat{v}^{2}} \hat{v},
$$

em que $\hat{V}=\|\hat{v}\|^{2}$ é o valor eficaz (norma) da integral imparcial da tensão.

De acordo com $i_{r}$ têm-se um comportamento indutivo conforme equação (4.15), bem como um comportamento capacitivo conforme (4.16),

$$
\begin{gathered}
B_{e}=\frac{W_{r}}{\hat{v}^{2}}, \\
C_{e}=\frac{\left|W_{r}\right|}{V^{2}},
\end{gathered}
$$

em que $B_{e}$ é a reatividade equivalente, bem como $C_{e}$ é a capacitância equivalente, de modo que nenhuma produz potência ativa. Da mesma forma, de acordo com as propriedades, temos $I_{r}=\left\|i_{r}\right\|$ sendo o valor eficaz da corrente reativa, a corrente reativa transporta toda a energia reativa e a potência ativa é nula, neste caso de acordo com as equações (4.17), (4.18), (4.19), (4.20) e (4.21). Além disso, a energia reativa é proporcional à diferença entre a energia média total indutiva e capacitiva,

$$
W_{r L}=\left\langle\hat{v}, i_{r}\right\rangle=B_{e}\langle\hat{v}, \hat{v}\rangle=B_{e}\|\hat{v}\|^{2}=B_{e} \hat{v}^{2}=W_{r}
$$




$$
\begin{gathered}
W_{r C}=\left\langle\hat{v}, i_{r}\right\rangle=C_{e} \frac{V^{2}}{\hat{v}}\langle\hat{v}, \hat{v}\rangle=C_{e} \frac{V^{2}}{\hat{v}^{2}}\|\hat{v}\|^{2}=C_{e} \hat{v}^{2}=\left|W_{r}\right|, \\
\left\|i_{r}\right\|=I_{r}=\frac{W_{r}}{\hat{v}} \\
P_{L}=\left\langle v, i_{r}\right\rangle=B_{e}\langle v, \hat{v}\rangle=0, \\
P_{c}=\left\langle v, i_{r}\right\rangle=C_{e} \frac{V^{2}}{\hat{v}^{2}}\langle v, \hat{v}\rangle=0 .
\end{gathered}
$$

\subsubsection{Corrente residual (nula)}

A corrente residual (nula) $i_{v}$ é

$$
i_{v}=i-i_{a}-i_{r}
$$

Esta corrente residual (nula) não transporta potência ativa e energia reativa. Os produtos internos resultam zero

$$
\begin{gathered}
P_{v}=\left\langle v, i_{v}\right\rangle=\left\langle v, i-i_{a}-i_{r}\right\rangle=\langle v, i\rangle-\left\langle v, i_{a}\right\rangle-\left\langle v, i_{r}\right\rangle=P-P_{a}-P_{L}=0, \\
W_{r v}=\left\langle\hat{v}, i_{v}\right\rangle=\left\langle\hat{v}, i-i_{a}-i_{r}\right\rangle=\langle\hat{v}, i\rangle-\left\langle\hat{v}, i_{a}\right\rangle-\left\langle\hat{v}, i_{r}\right\rangle=W_{r}-W_{r a}-W_{r L}=0 .
\end{gathered}
$$

O conjunto dos índices correspondentes aos harmônicos existentes na corrente $i$ e o conjunto de harmônicos da tensão $v$,

$$
\begin{gathered}
i=\sum_{k \in\left\{K_{i}\right\}} i_{k}=\sum_{k \in\left\{K_{i}\right\}} \sqrt{2} I_{k} \operatorname{sen}\left(k \omega t+\beta_{k}\right), \\
v=\sum_{k \in\left\{K_{v}\right\}} v_{k}=\sum_{k \in\left\{K_{v}\right\}} \sqrt{2} V_{k} \operatorname{sen}\left(k \omega t+\alpha_{k}\right) .
\end{gathered}
$$

A integral imparcial da tensão é 


$$
\hat{v}=\sum_{k \in\left\{K_{v}\right\}} \hat{v}_{k}=-\sum_{k \in\left\{K_{v}\right\}} \frac{\sqrt{2}}{\omega t} V_{k} \cos \left(k \omega t+\alpha_{k}\right) .
$$

O seu valor eficaz resulta

$$
\hat{v}^{2}=\sum_{k \in\left\{K_{v}\right\}} \hat{v}_{k}^{2}=\sum_{k \in\left\{K_{v}\right\}} \frac{V_{k}^{2}}{\omega^{2} k^{2}}
$$

Assim considerando uma única harmônica, o resultado é

$$
\hat{v}_{k}=\frac{V_{k}}{\omega k}
$$

em que $V_{k}$ e $\hat{v}_{k}$ representam o valor eficaz da k-ésima harmônica da tensão e integral parcial da tensão.

O conjunto de harmônicas comuns definido por $\{K\}$, ou seja, $K=\left\{K_{i} \cap K_{v}\right\}$, assim temos

$$
i=\sum_{k \in\{K\}} i_{k}=\sum_{k \in\left\{K_{i}-K\right\}} i_{k}=i_{h}+i_{g}
$$

Assim, $i_{h}$ contém todos os harmônicos de correntes coexistentes com as harmônicas de tensão, ao mesmo tempo em que $i_{g}$ são harmônicas que existem somente na corrente e são as harmônicas geradas na carga na qual representam as não linearidades da carga.

Pode-se definir a corrente ativa e reativa para cada componente harmônica $i_{h}$ como

$$
i_{a k}=\frac{\left\langle v, i_{k}\right\rangle}{\left\|v_{k}\right\|^{2}} v_{k}=\frac{P_{k}}{V_{k}^{2}} v_{k}=G_{k} v_{k}
$$




$$
i_{r k}=\frac{\left\langle\hat{v}_{k}, i_{k}\right\rangle}{\left\|\hat{v}_{k}\right\|^{2}} \hat{v}_{k}=\frac{\omega k^{2} W_{r k}}{V_{k}^{2}} \hat{v}_{k}=B_{k} \hat{v}_{k},
$$

assumindo, $\varphi_{k}=\alpha_{k}-\beta_{k}$, temos

$$
\begin{gathered}
P_{k}=V_{k} I_{k} \cos \varphi_{k}=G_{k} V_{k}^{2} \rightarrow G_{k}=\frac{I_{k}}{V_{k}} \cos \varphi_{k}, \\
W_{r k}=\frac{1}{\omega k} V_{k} I_{k} \operatorname{sen} \varphi_{k}=\frac{V_{k}^{2} B_{k}}{\omega^{2} k^{2}} \rightarrow B_{k}=\frac{\omega k I_{k}}{V_{k}} \operatorname{sen} \varphi_{k} .
\end{gathered}
$$

Neste caso, são associadas a cada única harmônica as somas da potência ativa total e a energia reativa total

$$
\begin{gathered}
\sum_{k \in\{K\}} P_{k}=P \\
\sum_{k \in\{K\}} W_{r k}=W_{r} .
\end{gathered}
$$

Dessa forma, a decomposição da corrente total assume

$$
i=\sum_{k \in\{K\}}\left(i_{a k}+i_{r k}\right)+\sum_{k \in\left\{K_{i}-K\right\}} i_{g}=i_{a h}+i_{r h}+i_{g}
$$

em que $i_{a h}$ é a corrente harmônica ativa total e $i_{r h}$ é a corrente harmônica reativa total. Neste contexto, essas representações de corrente, podem não ajustar-se com as definições de corrente ativa $i_{a}$ e reativa $i_{r}$. Portanto, podem ser definidos os termos de correntes dispersas. 
a) Corrente ativa dispersa

$$
i_{a s}=i_{a h}-i_{a}=\sum_{k \in\{K\}} \frac{P_{k}}{V_{k}^{2}} v_{k}-\frac{P}{V^{2}} v=\sum_{k \in\{K\}}\left(G_{k}-G_{e}\right) v_{k} .
$$

O valor eficaz da corrente ativa dispersa,

$$
I_{a s}=\left\|i_{a s}\right\|=\sqrt{\sum_{k \in\{K\}}\left(G_{k}-G_{e}\right)^{2} V_{k}^{2}} .
$$

A potência ativa total é gerada pela corrente ativa harmônica e não tem capacidade de gerar energia reativa harmônica. A componente ativa dispersa não gera potência ativa nem reativa.

b) Corrente reativa dispersa

$$
i_{r s}=i_{h r}-i_{r}=\sum_{k \in\{K\}} \frac{W_{r r k}}{\hat{v}_{k}^{2}} \hat{v}_{k}-\frac{W_{r}}{\hat{v}^{2}} \hat{v}=\sum_{k \in\{K\}}\left(B_{k}-B_{e}\right) \hat{v}_{k}
$$

O valor eficaz da corrente reativa dispersa

$$
I_{r s}=\left\|i_{r s}\right\|=\sqrt{\sum_{k \in\{K\}}\left(B_{k}-B_{e}\right)^{2 \hat{\imath} v_{k}^{2}}}
$$

A energia reativa total é decorrente da corrente reativa harmônica e não tem capacidade de gerar potência ativa harmônica. A componente reativa dispersa não gera potência ativa nem energia reativa.

De tal forma como no sistema físico, a corrente residual (nula) têm-se as correntes dispersas $i_{a s}$ e $i_{r s}$ na qual são devidas aos diferentes comportamentos que a condutância e a reatividade desempenham em diferentes frequências. Assim, os termos $i_{a s}$ e $i_{r s}$ devem-se ao fato que, em geral, $G_{k} \neq G_{e}$ e $B_{k} \neq B_{e}$.

Substituindo as equações (4.38) e (4.40) em (4.37), 


$$
i=i_{a}+i_{r}+i_{a s}+i_{r s}+i_{g}
$$

De acordo com a equação (4.22) que representa a corrente residual (nula), também pode ser decomposta em

$$
i_{v}=i_{a s}+i_{r s}+i_{g}
$$

e o termo auto-gerado $i_{g}$ pode ser obtido a partir da equação (4.43) e ser chamado de corrente harmônica gerada pela carga.

c) Corrente harmônica gerada pela carga

A corrente gerada pela carga,

$$
i_{g}=i_{v}-i_{a s}-i_{r s}
$$

A corrente harmônica gerada pela carga existe somente na corrente, na qual representam as linearidades da carga.

\subsection{Decomposição completa da corrente em circuitos monofásicos}

Todas as parcelas de corrente apresentadas no sub-item 4.3 são ortogonais entre si

$$
I^{2}=I_{a}^{2}+I_{r}^{2}+I_{v}^{2}=I_{a}^{2}+I_{r}^{2}+I_{a s}^{2}+I_{r s}^{2}+I_{g}^{2}
$$

De acordo com uma condição geral, a corrente total pode ser decomposta em:

- Corrente ativa $i_{a}$ : conversão constante de energia útil;

- Corrente reativa $i_{r}$ : deslocamento de fase entre tensão e corrente provocado pela presença de elementos passivos armazenadores de energia (indutores e 
capacitores) bem como pela presença de circuitos eletrônicos defasadores de corrente;

- Corrente dispersa $i_{s}=i_{a s}+i_{r s}$ : diferentes valores de condutância e reatividade em diferentes frequências;

- Corrente harmônica gerada pela carga $i_{g}$ : não linearidades da carga (harmônicos que não existem no espectro de tensão).

\subsection{Potência aparente em circuitos monofásicos}

A decomposição de potência pode ser obtida, válida para condições senoidal e não senoidal de acordo com os termos de corrente definidos nos itens anteriores. Assim, a análise de frequência também se torna possível. De acordo com a potência ativa $P$ especificada na equação (4.6), e seu valor da tensão eficaz da fonte de alimentação

$$
V=\sqrt{\langle v, v\rangle}=\frac{1}{T} \int_{0}^{T} v^{2} d t=\sqrt{\sum_{k=1}^{\infty} V_{k}^{2}},
$$

e o valor eficaz (norma) da corrente da fonte é igual a

$$
I=\sqrt{\langle i, i\rangle}=\frac{1}{T} \int_{0}^{T} i^{2} d t=\sqrt{\sum_{k=1}^{\infty} I_{k}^{2}} .
$$

De acordo com o valor eficaz (norma) da integral imparcial da tensão da equação (4.29), onde w é a frequência angular, $D H T_{V}$ é a distorção harmônica total e $D H T_{\hat{v}}$ é o fator de distorção harmônica total ponderada da tensão

$$
\frac{V}{\hat{v}}=\omega \sqrt{\frac{1+D H T_{V}^{2}}{1+D H T_{\hat{v}}^{2}}} .
$$


Definindo o fator de distorção da tensão como sendo

$$
\sigma_{v}=\sqrt{\frac{1+D H T_{v}^{2}}{1+D H T_{\hat{v}}^{2}}}-1,
$$

é possível encontrar

$$
\frac{V}{\hat{v}}=\omega\left(1+\sigma_{v}\right)
$$

Tendo $D H T_{V}^{2}>D H T_{\hat{v}}^{2}$, o fator de distorção da tensão é sempre maior que zero $\sigma_{v}>0$. A norma da integral imparcial da tensão também é influenciada pela variação da frequência de acordo com a equação (4.50).

A potência da fonte de alimentação é caracterizada pelo produto dos valores eficazes de tensão e corrente, denominada potência aparente,

$$
\|v\|\|i\|=V I=A
$$

Além disso, é um termo não conservativo na qual é relevante apenas para analisar e/ou dimensionar um circuito ou uma rede elétrica.

De acordo com as equações (4.51) e (4.45), a potência aparente pode ser decomposta em relação aos vários termos de corrente

$$
A^{2}=V^{2} I^{2}=V^{2} I_{a}^{2}+V^{2} I_{r}^{2}+V^{2} I_{a s}^{2}+V^{2} I_{r s}^{2}+V^{2} I_{g}^{2}
$$

Assim, a potência aparente pode ser decomposta em

$$
A^{2}=P^{2}+Q^{2}+D_{a}^{2}+D_{r}^{2}+D_{g}^{2}=P^{2}+Q^{2}+D^{2},
$$

em que:
$P$ : Potência ativa [W]
$Q$ : Potência reativa [Q]
$D$ : Potência residual (nula) [VA] 
$D_{a}$ : Potência ativa dispersa [VA]

$D_{r}$ : Potência reativa dispersa [VA]

$D_{g}$ : Potência harmônica gerada [VA]

$A$ : Potência aparente [VA]

\subsubsection{Potência ativa}

A potência ativa pode ser representada conforme a equação (4.54), sendo denominada a partir da corrente ativa

$$
P=V I_{a}=G_{e} V^{2}
$$

\subsubsection{Potência reativa}

A potência reativa é

$$
Q=V I_{r}=V \frac{W r}{\hat{v}}=\omega W_{r}\left(1+\sigma_{v}\right) .
$$

Arranjando-a em função de $\omega_{n}$ a frequência nominal de linha, pode ser calculada como

$$
Q=\omega_{n} W_{r} \frac{\omega}{\omega_{n}}\left(1+\sigma_{v}\right)=\omega_{n} W_{r}\left(1+\frac{\Delta \omega}{\omega_{n}}\right)\left(1+\sigma_{v}\right)
$$

Assim, $\Delta \omega=\omega-\omega_{n}$ pode ser positivo ou negativo, sendo a energia reativa $W$, e é um termo conservativo, bem como a potência reativa $Q$ não é um termo conservativo. O termo $Q$ é afetado pela distorção de tensão e pela variação da frequência da linha. 


\subsubsection{Potência residual (nula)}

Este termo chamado de potência residual (nula), é uma componente de corrente que não transporta nem potência ativa, nem energia reativa

$$
D=V I_{v}
$$

Esta potência residual (nula) está presente somente nas linearidades entre as ondas da tensão e corrente e/ou variações dos parâmetros passivos ( $G$ e $B$ ) do circuito com a frequência (dispersão).

a) Potência ativa dispersa

A potência ativa dispersa é

$$
D_{a}=V I_{a s}=\sqrt{V^{2} \sum_{k \in\{K\}}\left(G_{k}-G_{e}\right)^{2} V_{k}^{2}}
$$

em que $D_{a}$ é afetado pelas tensões harmônicas que são comuns com as correntes. Em caso de tensão senoidal, não se tem potência ativa dispersa

$$
V_{k}^{2}=0 \rightarrow D_{a}=0
$$

b) Potência reativa dispersa

A potência reativa dispersa é

$$
D_{r}=V I_{r s}=\omega_{r}\left(1+\frac{\Delta \omega}{\omega_{r}}\right)\left(1+\sigma_{v}\right) \sqrt{V^{2} \sum_{k \in\{K\}}\left(B_{k}-B_{e}\right)^{2} \hat{v}_{k}^{2}}
$$

o termo $D_{r}$ desaparece se a tensão é senoidal,

$$
V_{k}^{2}=0 \rightarrow D_{r}=0
$$


c) Potência harmônica gerada

A potência harmônica gerada é

$$
D_{g}=V I_{g}
$$

A potência harmônica gerada está presente somente quando a carga gera harmônicos de corrente distintas da tensão.

\subsection{Fator de potência em circuitos monofásicos}

O fator de potência é dado pela relação entre as potências ativa e aparente, conforme

$$
\lambda=\frac{P}{A},
$$

de (4.63) e (4.53) é encontrado

$$
\lambda=\frac{P}{\sqrt{P^{2}+Q^{2}+D^{2}}}=\frac{\left\|i_{a}\right\|}{\sqrt{\left\|i_{a}\right\|^{2}+\left\|i_{r}\right\|^{2}+\left\|i_{v}\right\|^{2}}},
$$

as correntes, reativa e residual, contribuem para a redução do fator de potência pela redução das mesmas.

\subsubsection{Fator de não linearidade}

O fator de não linearidade $\lambda_{D}$ é a relação entre a potência residual e a potência aparente, conforme

$$
\lambda_{D}=\frac{D}{\sqrt{P^{2}+Q^{2}+D^{2}}}=\frac{D}{A}=\frac{\left\|i_{v}\right\|}{\|i\|} .
$$




\subsubsection{Fator de reatividade}

O fator de reatividade $\lambda_{Q}$ é

$$
\lambda_{Q}=\frac{Q}{\sqrt{P^{2}+Q^{2}}}=\frac{\left\|i_{r}\right\|}{\sqrt{\left\|i_{a}\right\|^{2}+\left\|i_{r}\right\|^{2}}},
$$

assim, o fator de potência pode ser expresso por

$$
\lambda=\sqrt{\left(1-\lambda_{Q}^{2}\right)\left(1-\lambda_{D}^{2}\right)}=\sqrt{\left(1-\frac{Q^{2}}{P^{2}+Q^{2}}\right)\left(1-\frac{D^{2}}{P^{2}+Q^{2}+D^{2}}\right)}=\frac{P}{A}=\frac{\left\|i_{a}\right\|}{\|i\|} .
$$

\subsection{Considerações finais do capítulo}

Neste capítulo foi apresentado uma abordagem sobre a Compensação Cooperativa baseada na Teoria da Potência Conservativa de forma geral para tensões, correntes e definição de termos de potência e energia por fase, em condições senoidais, não senoidais e/ou assimétricas, desenvolvida no domínio do tempo e é aplicado para todos os tipos de rede. 


\section{Descrição dos Modelos Matemáticos e Projeto dos Controladores}

Este capítulo apresenta as modelagens do UPQC monofásico com compensação ativa de potência, série e paralela no referencial abc. Além disso, busca-se, obter as funções de transferência que representam o princípio de funcionamento do sistema UPQC, utilizando a estratégia de controle dual, e considerando que os conversores série e paralelo são controlados como fontes de corrente e tensão senoidais, ou seja, forma dual (Silva et al., 2002; Barriviera et al., 2012; Aredes et al.,2014; Modesto et al., 2015). Exibem-se ainda as análises matemáticas das malhas de controle dos conversores série e paralelo, como fontes ideais de corrente e de tensão, bem como a modelagem matemática do elo CC e da malha de controle, considerando a tensão total e o desequilíbrio, projeto dos controladores, além do fluxo de potência e a análise de estabilidade dos conversores série e paralelo.

\subsection{Introdução}

O circuito equivalente monofásico mostrado na Figura 5.1 apresenta o sistema UPQC. O conversor série de potência, representado por uma fonte de corrente senoidal, terá a capacidade de estabelecer uma corrente senoidal no circuito elétrico, bem como o conversor paralelo de potência, representado por uma fonte de tensão senoidal, irá impor tensão senoidal sem depender do tipo de carga conectada em seus terminais.

Figura 5.1 - Circuito monofásico.

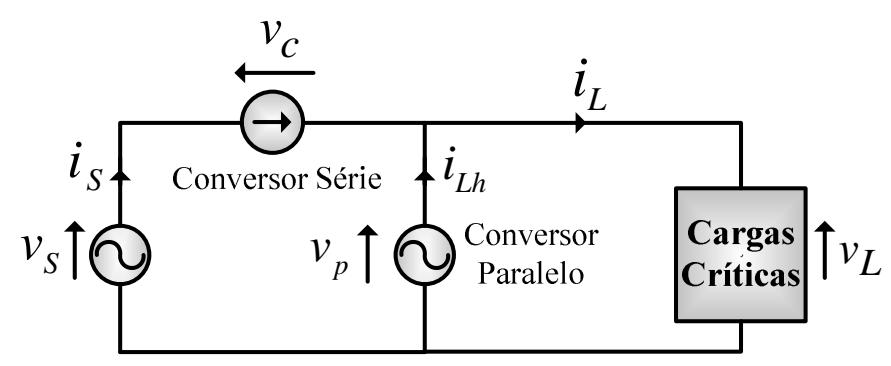

Fonte: Dados da pesquisa do autor. 
A tensão da rede elétrica $v_{s}$, considerando que seja distorcida, é constituída por uma parcela fundamental $v_{s f}$ mais a totalidade das componentes harmônicas $v_{s h}$ , de acordo com

$$
v_{s}=v_{s f}+\sum_{h}^{\infty} v_{s h} .
$$

O conversor paralelo de potência estabelece uma tensão sobre seus terminais de acordo com a tensão da rede elétrica $v_{s f}$, ou seja, com a mesma forma e amplitude da parcela fundamental. Assim, a tensão da carga $v_{L}$ será idêntica à tensão da rede $v_{s f}$. Com isso, nos terminais da fonte de corrente senoidal $v_{c}$ irão surgir as componentes harmônicas da tensão da rede elétrica $v_{s h}$, ou seja, as distorções de tensão estarão presentes no transformador de acoplamento série, que são compensados de forma indireta.

Quando uma carga não linear é acoplada a UPQC, a corrente da carga $i_{L}$ é constituída por uma parcela fundamental $i_{L f}$ juntamente com as componentes harmônicas $i_{L h}$, de acordo com

$$
i_{L}=i_{L f}+\sum_{h}^{\infty} i_{L h}
$$

O conversor série de potência impõe uma corrente senoidal constituída somente pela parcela fundamental da corrente da carga $i_{L f}$, independente da tensão resultante sobre seus terminais, produzindo, assim, um caminho de alta impedância para as correntes harmônicas da carga $i_{L h}$ que circularão no conversor paralelo. Dessa forma, o conversor paralelo absorve as correntes harmônicas da carga $i_{L h}$ que representa um caminho de baixa impedância, isolando a rede elétrica da carga. 


\subsection{Modelagem do Conversor Série}

Seja o conversor estático de potência série monofásico, que opera como uma fonte de corrente senoidal equilibrada, assim como o conversor estático de potência paralelo opera como fonte de tensão senoidal ideal, mostrado na Figura 5.2. Assumese que a tensão total do elo CC $V_{c c}$ é constante para um determinado período de comutação.

Figura 5.2 - Circuito monofásico do conversor série de corrente tipo ANPC cinco níveis conectado em série com a rede elétrica.

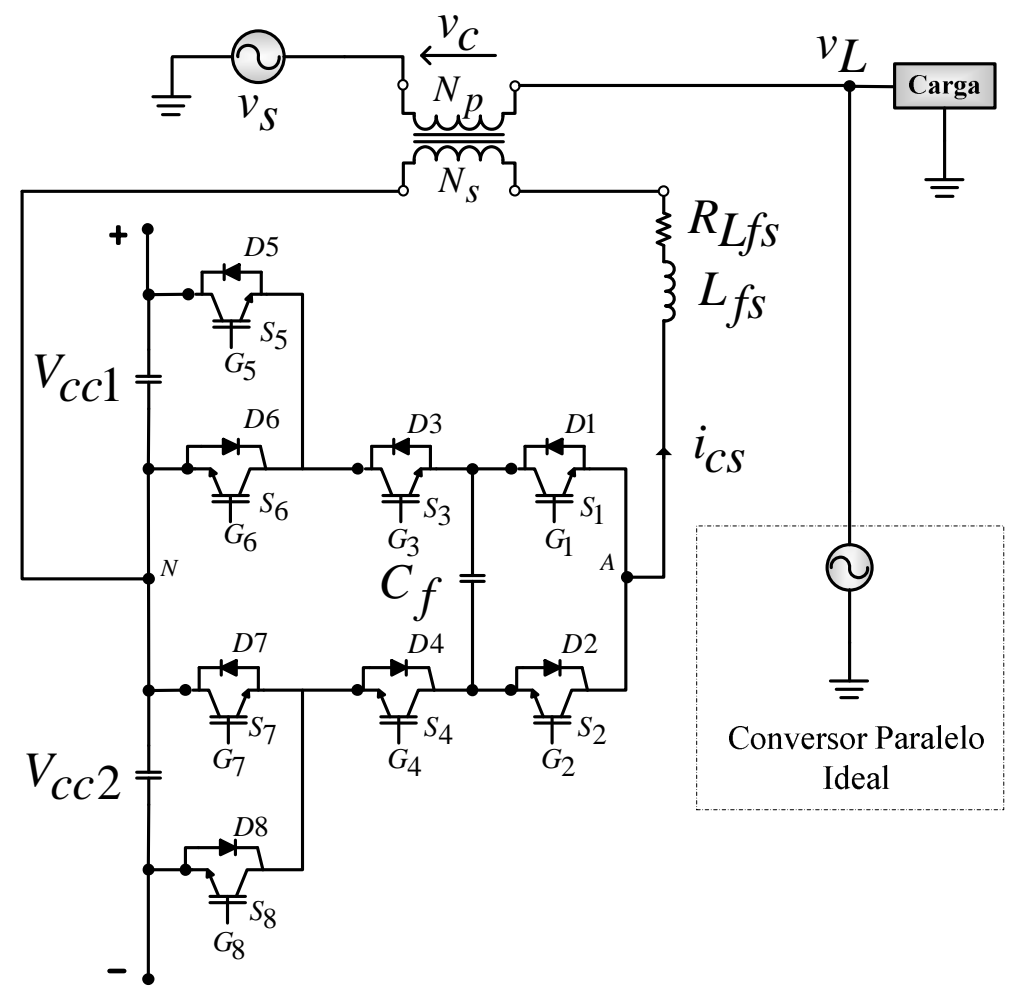

Fonte: Dados da pesquisa do autor.

O transformador de isolação, chamado também de transformador de acoplamento série, apresenta indutância de dispersão do primário $L_{d p}$ e do secundário $L_{d \mathrm{~s}}$, resistência do primário $R_{d p}$ e do secundário $R_{d s}$ (Fitzgerald et al., 2006; Santos, 2012). 
Figura 5.3 - Circuito equivalente considerando as indutâncias de dispersão e resistências do transformador de acoplamento série.

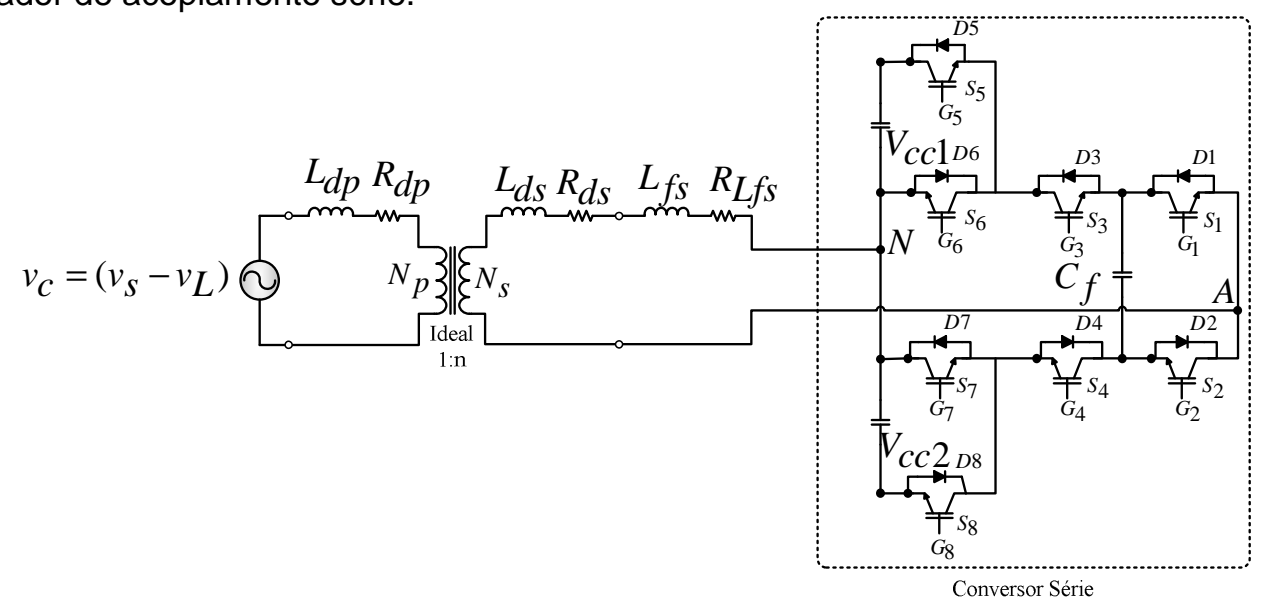

Fonte: Dados da pesquisa do autor.

Também é definido a indutância de acoplamento $L_{f s}$, bem como a resistência série equivalente $R_{L f s}$. Portanto, na Figura 5.3, é apresentado o circuito equivalente, no qual $v_{S}$ representa a tensão da rede elétrica e $v_{L}$ a tensão sobre a carga.

Considerando as equações (5.3), (5.4) e (5.5), bem como as indutâncias de dispersão e resistências totais, a tensão sobre o transformador de acoplamento série $v_{c}$ e as impedâncias são refletidas do lado secundário para o primário do transformador de isolação. Deste modo, é apresentado o circuito equivalente do conversor série, de acordo com a Figura 5.4.

$$
\begin{aligned}
n & =\frac{N_{p}}{N_{s}} \\
V_{\text {primário }} & =V_{\text {sec undário } \cdot n} \\
Z_{p} & =Z_{s} \cdot n^{2}
\end{aligned}
$$

De acordo com os pontos $\mathrm{A}$ e $\mathrm{N} v_{A N}$, em que são sintetizados os pulsos das chaves semicondutoras, com modulação a cinco níveis, a tensão varia de acordo com a matriz $\mathrm{R}$ descrita em (3.10). 
Figura 5.4 - Circuito equivalente do conversor série.

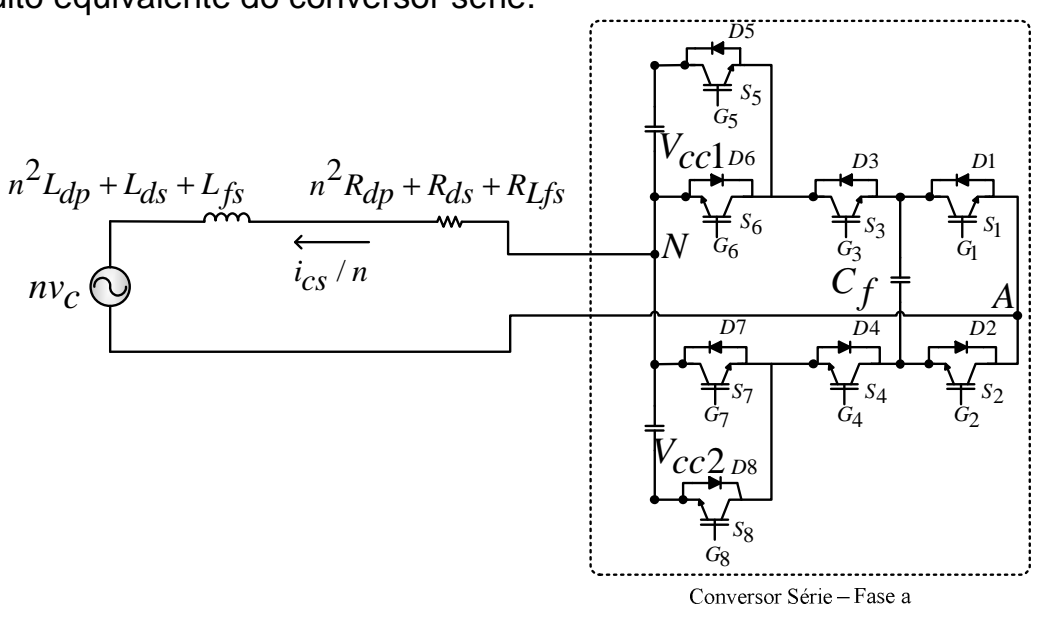

Fonte: Dados da pesquisa do autor.

Portanto, na Figura 5.5, tem-se o modelo do circuito equivalente do conversor série de acoplamento. A relação de transformação unitária do transformador é $n=1$, bem como $L_{e q_{s}}=L_{d p}+L_{d s}+L_{f s}$ e $R_{e q_{s}}=R_{d p}+R_{d s}+R_{f_{s}}$.

Figura 5.5 - Modelo do conversor série de potência.

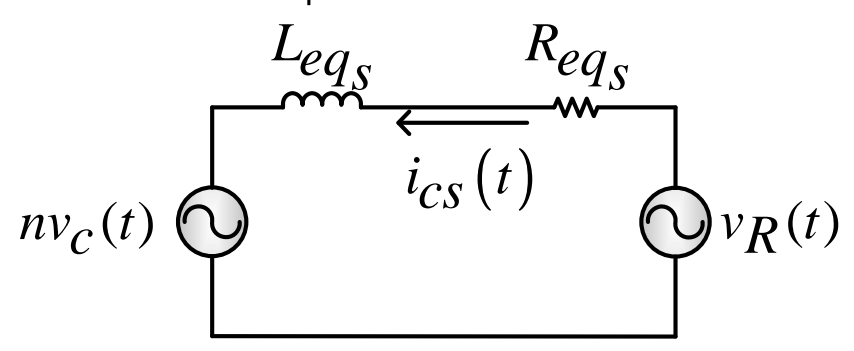

Fonte: Dados da pesquisa do autor.

De acordo com o modelo do conversor série, mostrado na Figura 5.5, tem-se a expressão

$$
v_{R}=v_{L_{e q}}+v_{R_{e q}}+v_{C}
$$

As quedas de tensão no indutor e no resistor são dadas por

$$
v_{L_{e q}}=L_{e q} \frac{d i_{c s}}{d t}
$$

e

$$
v_{R_{e q}}=R_{e q} i_{c s} .
$$


Substituindo as equações (5.7) e (5.8) em (5.6), obtém-se

$$
v_{R}=L_{e q} \frac{d i_{c S}}{d t}+R_{e q} i_{c s}+v_{C} .
$$

Rearranjando, encontra-se

$$
\frac{d i_{c s}}{d t}=\frac{1}{L_{e q}}\left(v_{R}-R_{e q} i_{c s}-v_{C}\right) .
$$

A equação de estado do sistema é dada como

$$
\dot{x}=A x+B U+F W,
$$

em que

$$
\begin{gathered}
x=\left[\begin{array}{ll}
i_{c S} & 0
\end{array}\right]^{T}, \\
U=\left[\begin{array}{ll}
V_{R} & 0
\end{array}\right]^{T}, \\
W=\left[\begin{array}{ll}
V_{C} & 0
\end{array}\right]^{T} .
\end{gathered}
$$

Substituindo (5.12), (5.13) e (5.14) em (5.11), e por meio de algumas manipulações matemáticas, a equação de estados da planta pode ser definida por

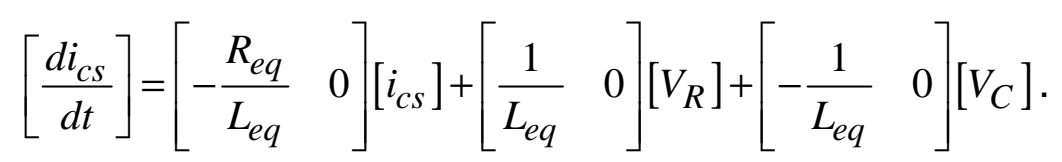

Ao aplicar a transformada de Laplace na equação (5.15), têm-se a função de transferência do conversor série, a partir do modelo apresentado na Figura 5.5, dada por

$$
G_{c s}=\frac{I_{c s}}{V_{R} D_{s}}=\frac{1}{L_{e q_{s}} s+R_{e q_{s}}} .
$$




\subsection{Modelagem da Malha de Controle de Corrente do conversor série}

A malha de controle de corrente do conversor série representado a partir de um diagrama de blocos, é mostrada na Figura 5.6, com o intuito de garantir que a corrente imposta pelo conversor série na rede elétrica seja a mais próxima possível da corrente de referência senoidal obtida pelo algoritmo de referência.

Figura 5.6 - Diagrama de blocos das malhas de controle de corrente do conversor série.

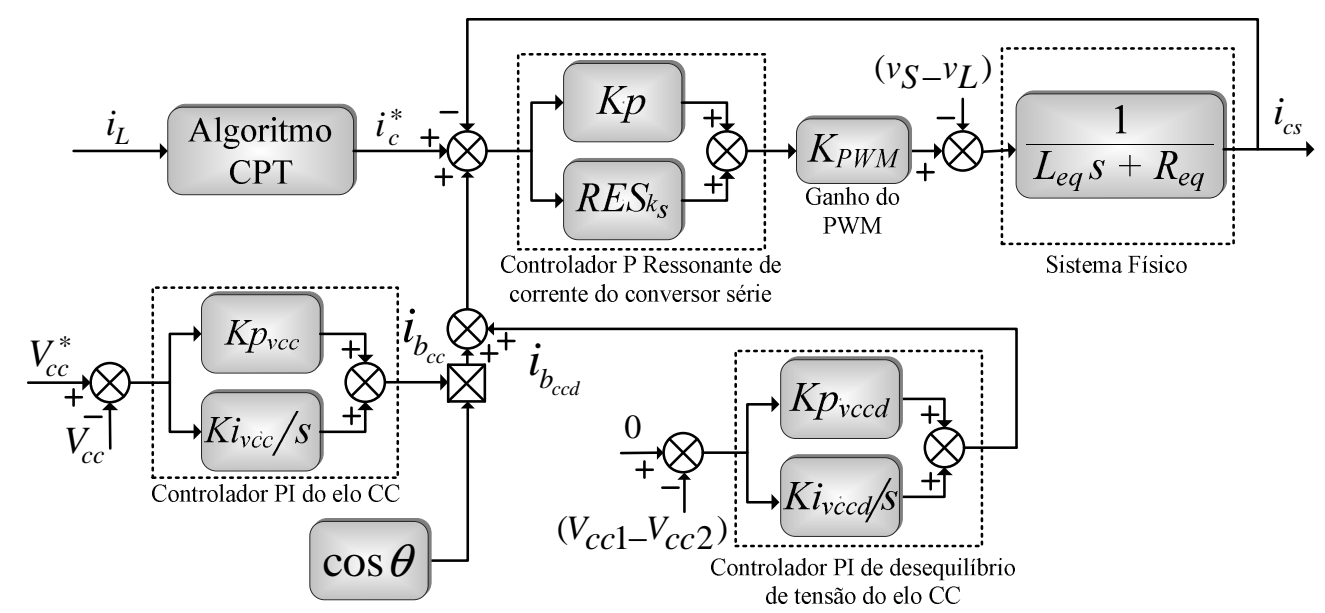

Fonte: Dados da pesquisa do autor.

De acordo com o diagrama de blocos da Figura 5.6, observa-se a malha de corrente, $K p_{s}$ e $R E S_{S}$ são os ganhos proporcional e ressonantes do controlador de corrente $G_{P+R E S_{C S}}$, conforme equação (5.17 e 5.18). Na equação (5.17), $k_{1_{k}}$ representa o ganho da função, que pode afetar a magnitude do sinal, e deve ser sintonizado de forma a minimizar o erro de regime permanente, $h_{k}$ é a ordem harmônica e $\omega_{0}$ é a frequência fundamental (Zmood; Holmes, 2003; Machado, 2005; Liserre; Teodorescu; Blaabjerg, 2006; Teodorescu et al., 2006; Buso; Mattavelli, 2006; Freijedo et al., 2011; Gazoli, 2011)

$$
\begin{gathered}
R E S_{k}=k_{1_{k}} \frac{s}{s^{2}+\left(h_{k} \omega_{0}\right)^{2}}, \\
G_{P_{C S}+R E S_{c s}}=k_{p}+\sum_{k=1,3, \ldots}^{n} R E S_{k} .
\end{gathered}
$$


O ganho do modulador PWM $K_{P W M}$, é calculado conforme a equação (5.19), em que $P_{P W M}$ é o valor de pico da portadora triangular (Buso; Mattavelli, 2006)

$$
K_{P W M}=\frac{1}{P_{P W M}}
$$

A fim de controlar o elo CC de tensão, de forma a compensar as perdas relacionadas aos dispositivos de comutação, bem como as indutâncias de filtragem, têm-se a malha de controle da tensão total e o desequilíbrio do elo CC $G_{P I_{v c c}}$ e $G_{P I_{v c c d}}$, também representado no diagrama de blocos da Figura 5.6, em que $K p_{v c c}$, $K p_{v c c d}$ e $K i_{v c c}$, Ki $i_{v c c d}$ são os ganhos proporcional e integral do controlador PI.

A função de transferência de malha aberta para a corrente do conversor série $G_{M A} C S$ é mostrado conforme o diagrama em blocos da Figura 5.7, bem como pelas equações (5.20) e (5.21).

Figura 5.7 - Diagrama em blocos de laço aberto de controle de corrente do conversor série

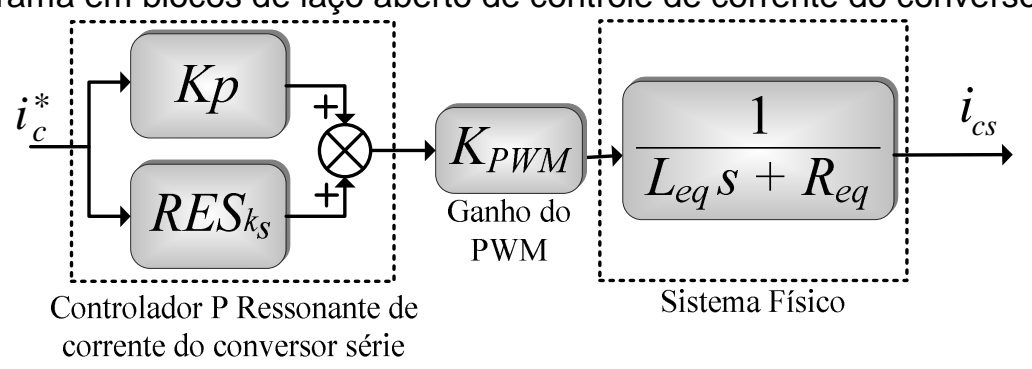

Fonte: Dados da pesquisa do autor.

$$
\begin{gathered}
G_{M A} C S=\frac{I_{c s}}{I_{c}^{*}}=G_{P_{C S}+R E S_{k}} K_{P W M} G_{C S} \\
G_{M A_{C S}}=\frac{I_{c s}}{I_{c}^{*}}=\frac{K_{P W M} R E S_{k} s+K_{P W M} K_{p}}{L_{e q_{s}} s+R_{e q_{s}}}
\end{gathered}
$$

A função de transferência em malha fechada que representa o conversor série $G_{M F_{C S}}$ de acordo com o diagrama em blocos da Figura 5.6, com ganho da malha de realimentação unitário, é dada por 


$$
G_{M F_{C S}}=\frac{I_{c s}}{I_{c}^{*}}=\frac{K_{P W M} R E S_{k} s+K_{P W M} K_{p}}{K_{P W M} R E S_{k} L_{e q_{s}} s+R_{e q_{s}}+K_{P W M} K_{p}}
$$

\subsection{Modelagem do Conversor Paralelo}

O circuito de potência do conversor paralelo de tensão é composto por um inversor monofásico multinível tipo ANPC de cinco níveis, conforme mostra a Figura 5.8 .

O conversor paralelo de tensão será modelado sem a influência direta das impedâncias da rede, da impedância de dispersão do transformador de acoplamento série e das impedâncias do conversor estático de potência série de corrente. Além disso, admite-se que a corrente do conversor série $i_{s}$ é uma fonte de corrente senoidal ideal e que as tensões senoidais de saída são reguladas. A diferença entre $\left(i_{L}-i_{S}\right)$ representa um distúrbio para a planta. Na saída do conversor paralelo de tensão, existe apenas um indutor $L_{f p}$, um resistor referente à resistência interna do indutor $R_{L f p}$, um capacitor $C_{f p}$ e a carga em paralelo com o capacitor de filtragem.

Figura 5.8 - Circuito trifásico do conversor paralelo de tensão tipo ANPC cinco níveis

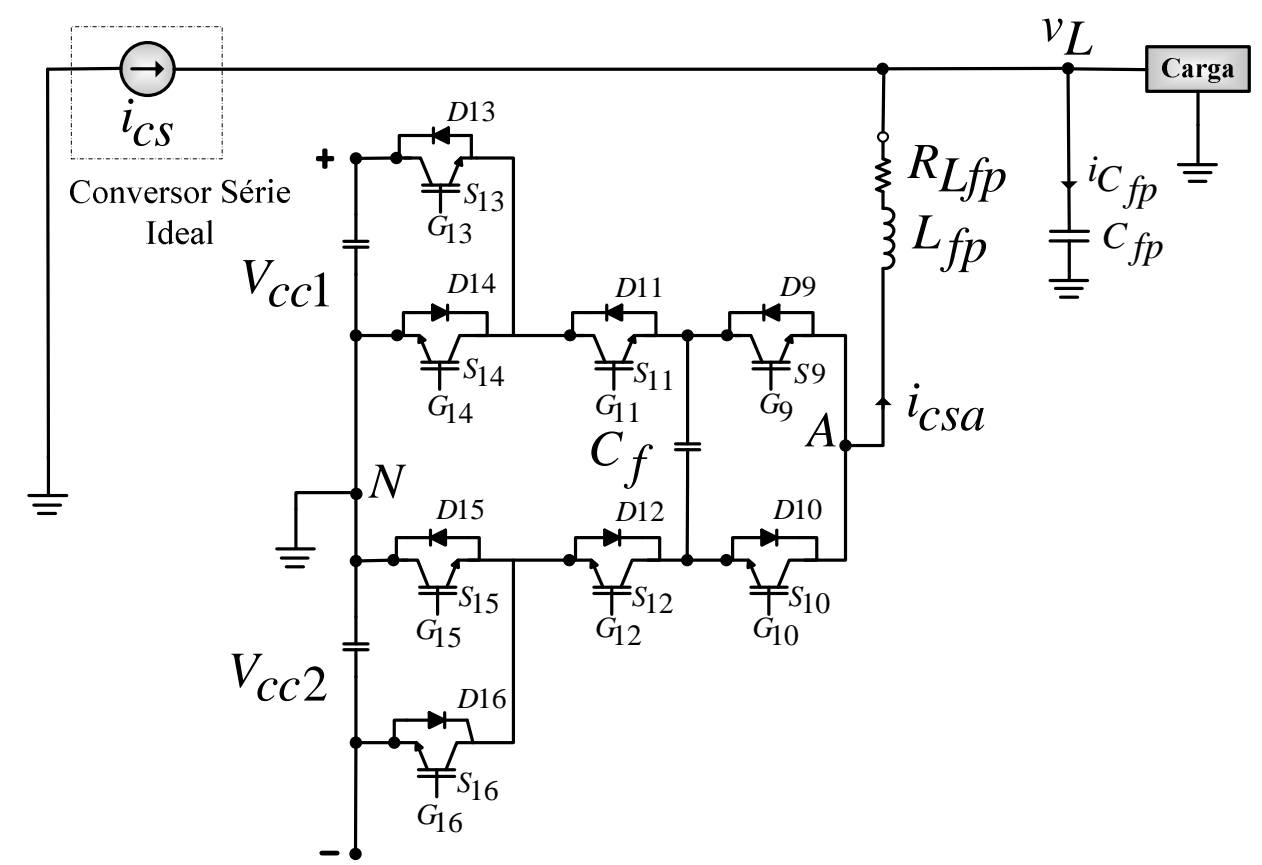

Fonte: Dados da pesquisa do autor. 
Os elementos de filtragem passivos são, $L_{f p}, R_{L f p}$ e $C_{f p}$, e suas respectivas representações, são conforme mostra a Figura 5.9.

Figura 5.9 - Circuito equivalente do conversor paralelo de tensão por fase

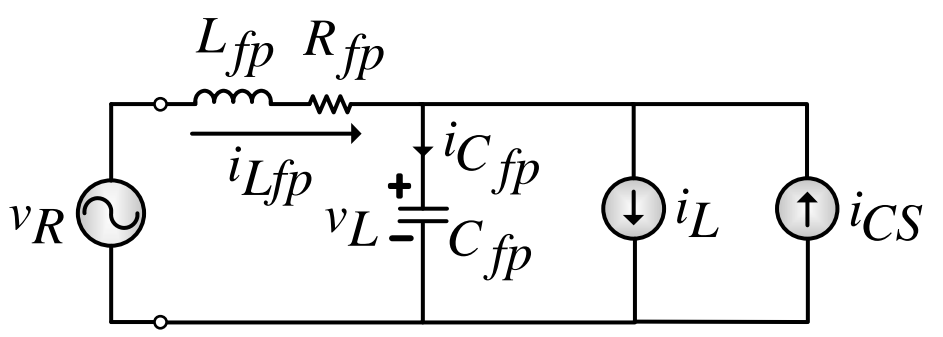

Fonte: Dados da pesquisa do autor.

Considerando a Figura 5.9, têm-se os valores médios das grandezas de tensão e de corrente, sendo: tensão na carga $v_{L}$, tensão sobre o capacitor $v_{C f p}$, tensão no resistor interno do indutor $v_{R_{L f p}}$, bem como a corrente no indutor ${ }_{L f p}$. Assim, a tensão equivalente do circuito é

$$
v_{R}=v_{L f p}+v_{R L f p}+v_{C f p}
$$

e a queda de tensão no indutor é

$$
v_{L f p}=L_{f p} \frac{d i_{L f p}}{d t} .
$$

Substituindo (5.22) em (5.21) e rearranjando, tem-se

$$
\frac{d i_{L f p}}{d t}=\frac{v_{R}}{L_{f p}}-\frac{R_{L f p} i_{L f p}}{L_{f p}}-\frac{v_{C f p}}{L_{f p}} .
$$

Considerando a corrente no indutor, tem-se

$$
i_{L f p}=C_{f p} \frac{d v_{C f p}(t)}{d t}=\frac{1}{C_{f p}} i_{L f p} .
$$


Por fim, a equação de estados do sistema pode ser definida conforme

$$
\left[\begin{array}{c}
\frac{d i_{L f p}}{d t} \\
\frac{d v_{C f p}}{d t}
\end{array}\right]=\left[\begin{array}{cc}
-\frac{R_{L f p}}{L_{f p}} & -\frac{1}{L_{f p}} \\
\frac{1}{C_{f p}} & 0
\end{array}\right]\left[\begin{array}{c}
i_{L f p} \\
v_{C f p}
\end{array}\right]+\left[\begin{array}{cc}
\frac{1}{L_{f p}} & 0 \\
0 & 0
\end{array}\right]\left[\begin{array}{c}
v_{R} \\
0
\end{array}\right]+\left[\begin{array}{ll}
0 & 1 \\
0 & 0
\end{array}\right]\left[\begin{array}{c}
i_{L f p} \\
v_{C f p}
\end{array}\right]
$$

Tem-se a função de transferência do conversor estático de potência paralelo $G_{C P}$, a partir da equação (5.27),

$$
G_{C P}=\frac{V_{L}}{V_{R} D_{p}}=\frac{1}{\left(L_{f p} C_{f p} s^{2}+R_{L f p} C_{f p} s+1\right)} .
$$

\subsection{Modelagem da Malha de Controle de Tensão do conversor paralelo}

De modo a garantir que a tensão imposta na carga seja senoidal e regulada, é implementada a malha de controle de tensão do conversor estático de potência paralelo multinível tipo ANPC cinco níveis, de acordo com o diagrama da Figura 5.10.

Este controlador é composto por duas malhas de controle, uma malha interna de corrente, responsável por ajustar a corrente do indutor de filtragem por meio de um regulador proporcional de corrente $P_{c i}$, com função de transferência definida por $G_{P_{c i}}$, e outra malha externa de tensão, que regula a tensão de saída do conversor paralelo de tensão por meio de um regulador proporcional ressonante de tensão $P_{R E S c v}$, de acordo com as equações (5.17 e 5.18), com função de transferência definida por $G_{P_{C v}}$, que fornece a referência de corrente $i_{L f p}^{*}$ para a malha interna. Para que a planta não entre em instabilidade, assume-se que a malha interna de corrente é muito mais rápida que a malha externa, de forma a ajustar os controladores destas malhas, compondo suas respectivas características. As entradas $i_{L}-i_{S}$ representam um distúrbio no sistema. 
Figura 5.10 - Diagrama de blocos das malhas de controle de tensão e corrente do conversor paralelo

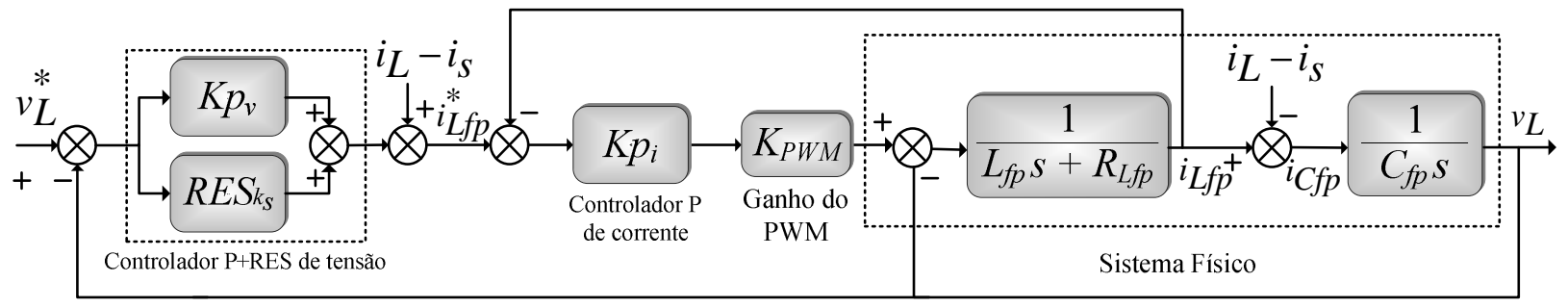

Fonte: Dados da pesquisa do autor.

De acordo com o diagrama de blocos da Figura 5.10, têm-se os controladores $G_{P_{c i}}$, da malha interna de corrente, e $G_{P_{c v}}$, da malha externa de tensão, conforme as equações (5.29) e (5.30).

$$
\begin{gathered}
G_{P_{c i}}=K_{p} i \\
G_{P_{c v}}=k_{p v}+\sum_{k=1,3, \ldots}^{n} R E S_{k} .
\end{gathered}
$$

A função de transferência de laço aberto da malha interna de corrente $G_{M A_{i}}$, é apresentada na Figura 5.11, de acordo com a equação (5.31) e (5.32), bem como a função de transferência de laço fechado da malha interna de corrente $G_{M F_{i}}$, ou seja, em malha fechada

$$
\begin{gathered}
G_{M A_{i}}=\frac{I_{L f p}}{I_{L f p}^{*}}=G_{P_{c i}} K_{P W M} G_{c p i}, \\
G_{M A_{i}}(s)=\frac{I_{L f p}}{I_{L f p}^{*}}=\frac{K p_{i} K_{P W M}}{L_{f p} s+R_{L f p}}, \\
G_{M F_{i}}(s)=\frac{I_{L f p}}{I_{L f p}^{*}}=\frac{K p_{i} K_{P W M}}{L_{f p} s+R_{L f p}+K p_{i} K_{P W M}} .
\end{gathered}
$$

Figura 5.11 - Diagrama de blocos da malha interna do controle de corrente do conversor paralelo de tensão

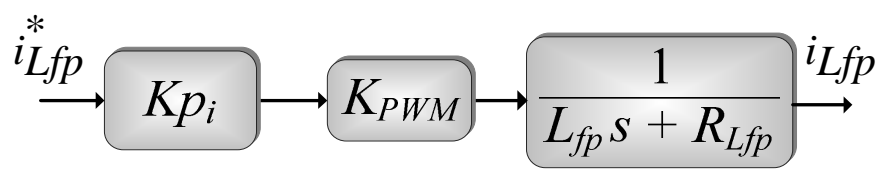

Fonte: Dados da pesquisa do autor. 
A função de transferência de laço aberto da malha externa de tensão $G_{M A_{v}}$, é mostrada na Figura 5.12, assim como é apresentada na equação (5.34).

Figura 5.12 - Diagrama de blocos da malha externa de tensão do conversor paralelo de tensão sem o controlador PI

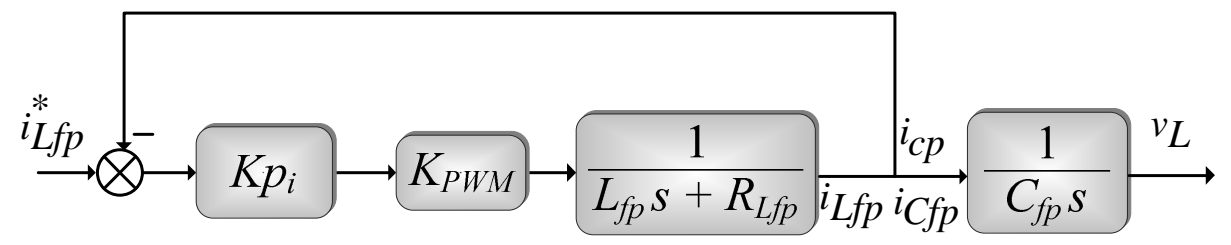

Fonte: Dados da pesquisa do autor.

$$
G_{M A_{v}}=\frac{v_{L}}{i_{L f p}^{*}}=\frac{K p_{i} K_{P W M}}{L_{f p} C_{f p} s^{2}+\left[C_{f p} K p_{i} K_{P W M}+C_{f p} R_{L f p}\right] s+1}
$$

O diagrama de blocos para a obtenção da função de transferência de laço aberto da malha externa de tensão do conversor paralelo de tensão, considerando a perturbação de carga nula e o controlador proporcional ressonante, é mostrada na Figura 5.13.

Figura 5.13 - Diagrama de blocos da malha externa de tensão do conversor paralelo de tensão com o controlador PI

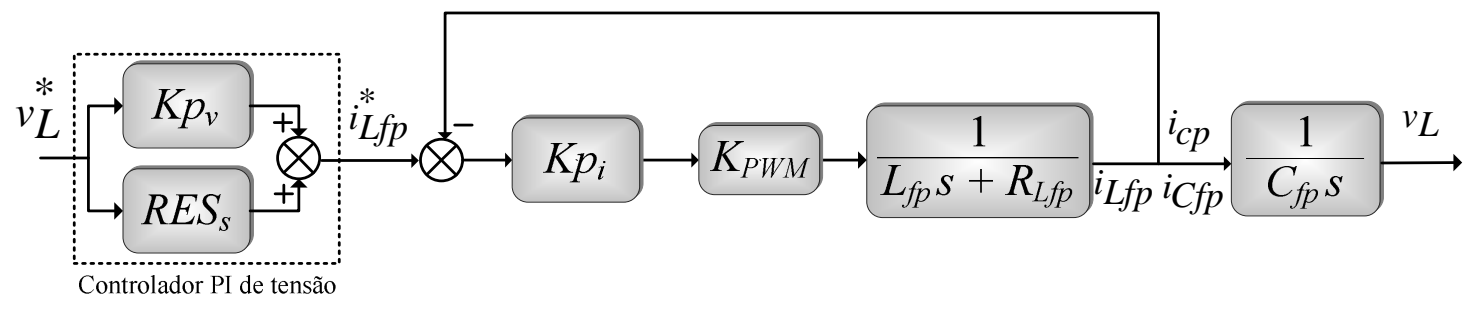

Fonte: Dados da pesquisa do autor.

As equações das funções de transferência em malha aberta e em malha fechada são dadas por

$$
G_{M A_{c v}}=\frac{v_{L}}{v_{L}^{*}}=\frac{R E S_{k} s K p_{i} K_{P W M}+K_{p v} K p_{i} K_{P W M}}{L_{f p} C_{f p} s^{2}+\left[C_{f p} K p_{i} K_{P W M}+C_{f p} R_{L f p}\right] s+1},
$$

em que $U=K p_{i} K_{P W M}$, e 


$$
G_{M F_{v}}=\frac{v_{L}}{v_{L}^{*}}=\frac{R E S_{k} U s+K_{p v} U}{L_{f p} C_{f p} s^{2}+\left[C_{f p} U+C_{f p} R_{L f p}+R E S_{k} U\right] s+K_{p v} U+1} .
$$

\subsection{Modelagem do Elo CC}

Com o intuito de se obter a função de transferência referente ao modelo do elo $\mathrm{CC}$, realiza-se a modelagem matemática para o projeto dos ganhos dos controladores. A partir do conversor monofásico, considerando que $v_{s}$ e $i_{s}$ constituem a tensão e a corrente senoidal de entrada, a potência ativa instantânea é

$$
p_{\text {in }}=v_{s} i_{s} .
$$

A potência ativa instantânea no referencial síncrono é dada na equação (5.38), em que $v_{d}$ e $i_{d}$ são representações de grandezas no referencial síncrono e índice $m$ representa as componentes médias (Silva, 2001)

$$
p_{\text {in }}=v_{d} i_{d}=v_{d m} i_{d m}=p_{m} .
$$

Considerando a corrente sem compensação no eixo síncrono, $i_{d L}=\left(i_{d L m}+i_{d L z}\right)$, em que 0 índice $z$ representa as componentes oscilantes, a potência ativa instantânea é

$$
p_{\text {out }}=v_{d}\left(i_{d L m}+i_{d L z}\right)
$$

De acordo com as equações (5.38) e (5.39), $i_{d L m}$ é igual a $i_{d m}$, e $p_{i n}$ é igual a $p_{m}$. Assim, a parcela de potência da rede elétrica para o conversor paralelo de tensão $p_{s c}$ é descrita como

$$
p_{\text {sc }}=p_{\text {in }}-p_{\text {out }} .
$$

De acordo com a parcela ativa de potência $p_{c m}$ que circula entre a rede elétrica e o conversor estático de potência paralelo de forma a compensar as perdas dos 
conversores série e paralelo, as parcelas de corrente ativa $i_{c m}$ e oscilante de compensação $i_{c z}$, que circulam pelo conversor paralelo, são

$$
p_{s c}=v_{d} i_{c m}+v_{d} i_{c z}=p_{c m}+p_{k}
$$

conforme $p_{c m}$ que é igual à potência no lado $\mathrm{CC} p_{c c}$, como

$$
p_{c m}=v_{d} i_{c m}=v_{c c} i_{c c}=p_{c c}
$$

em que $v_{c c}$ e $i_{c c}$ representam a tensão e a corrente do elo CC. Dessa maneira, a corrente $i_{c c}$ é mostrada de acordo com a equação (5.43) e (5.44) na qual o elo CC é representado por uma capacitância elevada $C_{c c}$,

$$
\begin{gathered}
i_{c c}=C_{c c} \frac{d_{v_{c c}}}{d t}, \\
i_{c c}=\frac{v_{d} i_{c m}}{v_{c c}} .
\end{gathered}
$$

Por meio de (5.43) e (5.44), tem-se

$$
\frac{d_{v_{c c}}}{d t}=\frac{v_{d} i_{c m}}{C_{c c} v_{c c}}
$$

Com a modelagem de pequenos sinais, em que considera-se os valores médios instantâneos das grandezas $v_{c c}$ e $i_{c m}$, uma vez que a variação de tensão do elo CC é pequena, realiza-se as manipulações matemáticas necessárias e aplica-se a Transformada de Laplace na equação (5.45). Com isso, tem-se a função de transferência da planta do elo CC, dada por

$$
G_{p_{v c c}}=\frac{\hat{v_{c c}}}{i_{c m}}=\frac{v_{d}}{C_{c c} V_{c c} s}
$$




\subsection{Modelagem da Malha de Controle do Elo CC}

O diagrama de blocos mostrado na Figura 5.14 apresenta a malha de controle de tensão do elo CC do UPQC. A principal finalidade desta malha de controle consiste em manter a tensão $V_{c c}$ constante de acordo com o valor de referência estabelecido no projeto. A tensão no elo $C C$ pode sofrer variações em função das diferenças entre as tensões de entrada e de saída do UPQC, perdas nos conversores estáticos de potência, elementos passivos de filtragem e fluxo de potência ativa entre a rede elétrica e o elo CC.

A resposta dinâmica do controlador precisa ser lenta de forma a atuar somente na amplitude de referência da corrente do conversor série, conforme diagrama de blocos da Figura 5.6, a fim de não interferir e distorcer a corrente de referência da malha de corrente do conversor série.

Figura 5.14 - Diagrama de bloco da malha de controle da tensão do elo CC

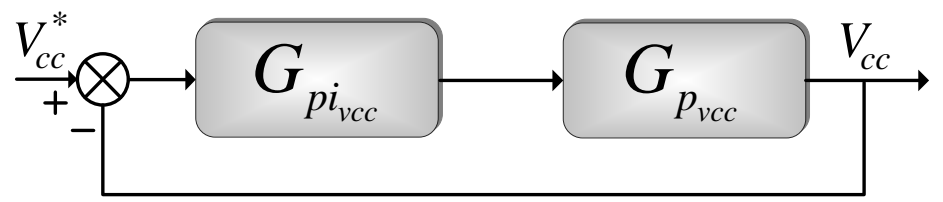

Fonte: Dados da pesquisa do autor.

A função de transferência do controlador PI do elo CC é

$$
G_{P I_{v c c}}=\frac{K p_{v c c} s+K i_{v c c}}{s} .
$$

Assim, de acordo com as equações (5.46) e (5.47), tem-se a função de transferência em malha aberta do elo CC na equação (5.48). Na equação (5.49), temse a função de transferência em malha fechada do elo CC, conforme a Figura 5.14.

$$
\begin{gathered}
G_{M A_{v c c}}=\frac{v_{d} K p_{v c c} s+v_{d} K i_{v c c}}{C_{c c} V_{c c} s^{2}} \\
G_{M F_{v c c}}=\frac{v_{d} K p_{v c c} s+v_{d} K i_{v c c}}{C_{c c} V_{c c} s^{2}+v_{d} K p_{v c c} s+v_{d} K i_{v c c}}
\end{gathered}
$$




\subsection{Modelagem do Desequilíbrio do Elo CC}

De modo a projetar o controlador do desequilíbrio do Elo CC apresentado na Figura 5.6, é necessário obter a função de transferência do desequilíbrio de tensão de acordo com o ponto médio do elo CC e a corrente de entrada do UPQC. De acordo com o circuito simplificado da Figura 5.15, obtém-se a função de transferência, de modo que são consideradas duas fontes de corrente que descrevem as correntes nas chaves de potência (Santos et. al., 2011 e 2014).

Figura 5.15 - Diagrama do circuito simplificado do desequilíbrio de tensão do elo CC

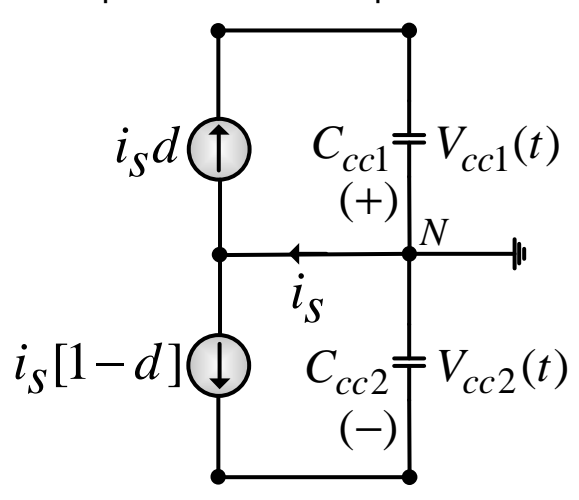

Fonte: Dados da pesquisa do autor.

As equações (5.50) e (5.51) representam as correntes nos capacitores $C_{c c 1} \mathrm{e}$ $C_{c c 2}$ de acordo com a Figura 5.15.

$$
\begin{gathered}
i_{s} d=C_{c c 1} \frac{d v_{c c 1}}{d t} \\
i_{S}[1-d]=C_{c c 2} \frac{d v_{c c 2}}{d t}
\end{gathered}
$$

De forma a obter a função de transferência do modelo de desequilíbrio de tensão para um sistema monofásico, aplica-se a Transformada de Laplace nas equações (5.50) e (5.51). Assim, tem-se

$$
\frac{v_{c c 1}}{i_{s}}-\frac{v_{c c 2}}{i_{s}}=\frac{D}{C_{c c 1} s}-\frac{(D-1)}{C_{c c 2^{2}}} .
$$


Considerando $C_{c c 1}=C_{c c 2}=2 C_{c c}$, a função de transferência da planta é

$$
G_{p_{v c c_{d}}}=\frac{1}{2 C_{c c}} \text {. }
$$

\subsection{Modelagem da Malha de Controle do Desequilíbrio de Tensão do Elo CC}

Na Figura 5.16, é mostrado o diagrama de blocos da malha de controle do desequilíbrio de tensão do elo CC. Sua finalidade é compensar os desequilíbrios de tensão entre os capacitores em relação ao ponto médio do elo CC.

Os desequilíbrios podem aparecer e aumentar em consequência da existência de componentes contínuas na tensão de saída e na corrente de entrada, de forma que aparecem em função dos diferentes tempos de comutação das chaves de potência dos braços dos conversores estáticos de potência.

De forma a compensar tais desequilíbrios, é utilizado um controlador proporcional integral, que atua na referência zero do conversor série de corrente. A ação de controle representa a referência de corrente no referencial zero, de tal modo que qualquer valor médio não nulo no referencial zero representa uma componente contínua no referencial abc.

No momento de ajustar os ganhos da malha de controle de desequilíbrio de tensão do elo CC, para que não se introduza componentes alternadas no referencial zero, que resultaria em correntes desequilibradas na entrada do UPQC, deve se ajustar a malha de controle de forma a ser mais lenta que a malha de controle de tensão do elo CC.

Figura 5.16 - Diagrama de bloco da malha de controle do desequilíbrio de tensão do elo CC

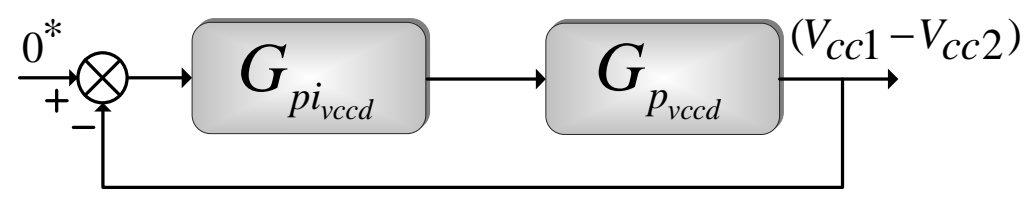

Fonte: Dados da pesquisa do autor.

A função de transferência do controlador PI do elo CC é 


$$
G_{P I_{v c c d}}=\frac{K p_{v c c d} s+K i_{v c c d}}{s} .
$$

Assim, de acordo com as equações (5.53) e (5.54), tem-se a função de transferência em malha aberta do elo CC na equação (5.55), bem como, na equação (5.56), tem-se a função de transferência em malha fechada do elo.

$$
\begin{gathered}
G_{M A_{v c c d}}=\frac{K p_{v c c d} s+K i_{v c c d}}{2 C_{c c} s^{2}} \\
G_{M F_{v c c d}}=\frac{K p_{v c c d} s+K i_{v c c d}}{2 C_{c c} s^{2}+K p_{v c c d} s+K i_{v c c d}}
\end{gathered}
$$

\subsection{Projeto dos controladores}

Os controladores utilizados na UPQC, consistem em controladores proporcional ressonante (P-RES), utilizados na malha de corrente do conversor série e outro na malha externa de tensão do conversor paralelo, e um controlador proporcional $(\mathrm{P})$ para a malha interna de corrente do conversor paralelo, bem como um controlador proporcional-integral $(\mathrm{Pl})$ para a malha de tensão total do elo $\mathrm{CC}$ e outro para a malha de tensão de desequilíbrio do elo CC.

\subsubsection{Projeto do controlador proporcional integral (PI)}

De forma a projetar os ganhos proporcional e integral dos controladores, serão consideradas a margem de fase $\varphi_{d}$ e a frequência de cruzamento $\omega_{c}$ da planta compensada. $\mathrm{O}$ amortecimento e a estabilidade da planta estão relacionados com a margem de fase $\varphi_{d}$, assim, quanto maior $\varphi_{d}$, maior é o amortecimento da planta (Silva, 2001). Para dispositivos chaveados, a margem de fase deve ficar entre $45^{\circ} \mathrm{e}$ 90ํ (Barbi, 2001; Silva, 2001; Angelico, et al. 2014). Na equação (5.57), é apresentada 
a função de transferência do controlador PI, com a presença de um zero e um pólo na origem,

$$
G_{c}=K p+\frac{K i}{s}=\frac{K p s+K i}{s}
$$

O tempo de resposta da planta é condicionado à frequência de cruzamento $\omega_{c}$ , de modo que, quanto maior é $\omega_{c}$, menor é o seu tempo de resposta. A limitação de $\omega_{c}$ está exatamente na frequência de comutação, de forma que as ondulações impostas pela comutação não interfiram no controle. Portanto, é recomendado que a $\omega_{c}$ fique entre um quarto e um décimo da frequência de chaveamento (Barbi, 2001).

De acordo com as equações (5.58) e (5.59), são obtidos os ganhos proporcional e integral dos controladores,

$$
\begin{gathered}
K p=M c \cos \phi_{c}, \\
K i=-K p \omega_{c} \operatorname{tg} \phi_{c},
\end{gathered}
$$

em que $M c$ é o módulo dos controladores PI na frequência de cruzamento $\omega_{c}$ desejada e na margem de fase $\phi_{c}$ que os controladores devem possuir. A margem de fase $\phi_{c}$ é calculada pela diferença entre a margem de fase desejada $\phi_{d}$ e a margem de fase da planta $\phi_{g}$, acrescentado mais $180^{\circ}$, de modo a assegurar que a planta compensada tenha um $\phi_{d}$ em $\omega_{c}$ especificado em projeto,

$$
\phi_{c}=\phi_{d}-\left(\phi_{g}+180^{\circ}\right)
$$

\subsubsection{Projeto do controlador proporcional ressonante (P-RES)}

De forma a realizar a compensação de sinais oscilatórios no referencial abc, é utilizado um controlador proporcional ressonante ( $P+R E S)$. O P-RES é a somatória de vários filtros ressonantes ajustados em uma determinada frequência préestabelecida em paralelo com um controlador $\mathrm{P}$. 
O princípio de funcionamento do P-RES, baseia-se em somar um alto ganho na frequência de referência pré-estabelecida a ser seguida, de modo a diminuir ou até mesmo eliminar o erro em regime. Assim, torna-se possível realizar a compensação de harmônicos pré-selecionados em conjunto com o processo de controle (Machado, 2005; Liserre, Teodorescu, Blaabjerg, 2006; Teodorescu et al., 2006; Buso, Mattavelli, 2006; Freijedo et al., 2011). Na Figura 5.17, apresenta-se a estrutura do P-RES, em que $\varepsilon$ é o erro de entrada e y é a saída.

Figura 5.17 - Diagrama de bloco do controlador Proporcional Ressonante (P-RES)

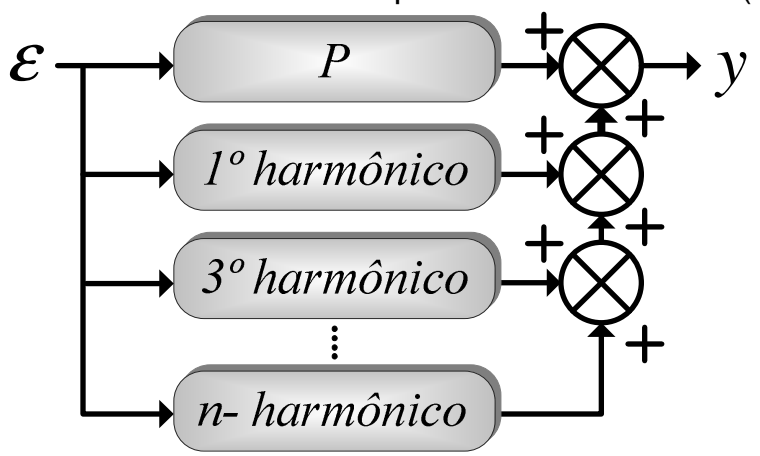

Fonte: Dados da pesquisa do autor.

Segundo (Buso; Mattavelli, 2006), a função de transferência do controlador $P+R E S$ é dada por

$$
\begin{gathered}
G_{P+R E S}=k_{p}+k_{R_{k}} \frac{s}{s^{2}+\left(h_{k} \omega_{0}\right)^{2}}, \\
G_{P+R E S}=k_{p}+\sum_{k=1,3, \ldots}^{n} R E S_{k},
\end{gathered}
$$

em que $k_{p}$ é o ganho proporcional, $k_{R_{k}}$ é o ganho ressonante da função a ser sintonizado de forma a minimizar o erro de regime permanente da ordem harmônica selecionada $h_{k}$, e $\omega_{0}$ é a frequência fundamental, que pode ser obtida a partir do cosseno do PLL (algoritmo de sincronismo).

Vale ressaltar que, quanto maior for $k_{R_{k}}$, maior será o ganho e o amortecimento na frequência pré-estabelecida, ou seja, o controlador será mais rápido, entretanto, a largura de banda será maior. Quando se tem uma largura de banda elevada, o controlador torna-se menos seletivo, e poderá implicar em um aumento de componentes harmônicas indesejáveis, contribuindo para o aumento da 
distorção harmônica do sinal de referência a ser compensado (Buso; Mattavelli, 2006; Vasquez et al., 2013; Blaadjerg et al., 2014).

Os ganhos dos controladores ressonantes são calculados de acordo com (5.61), sendo que os valores podem variar de acordo com as frequências préestabelecidas,

$$
k_{R_{k}}=\frac{2,2 k_{p}}{t_{r}}
$$

em que $t_{r}=n_{0} T_{0}$ é o tempo de resposta desejado para a frequência fundamental (valor varia entre $10 \%$ a $90 \%$ da resposta ao degrau), $T_{0}$ é o período da frequência fundamental e $n_{0}$ é o número de períodos de $T_{0}$ (Buso; Mattavelli, 2006).

\subsubsection{Projeto do controlador P-RES da malha de controle do conversor série}

A tabela 5.1 apresenta as especificações adotadas para o projeto do controlador P-RES utilizado na malha de corrente do conversor série, modelado na Seção 4.3.

Tabela 5.1 - Especificações para projeto do controlador P-RES de corrente do conversor série.

\begin{tabular}{ll}
\hline Indutância de acoplamento do conversor série & $L_{f_{s}}=4,3 \mathrm{mH}$ \\
Resistência série de acoplamento do conversor série & $R_{L_{f s}}=0,4 \Omega$ \\
Relação de transformação do transformador de acoplamento série & $n=1$ \\
Indutância de dispersão do transformador de acoplamento série & $L_{d t}=0,14 \mathrm{mH}$ \\
Resistência total de dispersão do transformador de acoplamento série & $R_{d t}=0,21 \Omega$ \\
Tensão elo CC total & $V_{c c_{\text {total }}}=250 \mathrm{~V}$ \\
Frequência de chaveamento do conversor de potência série & $f_{S}=20 \mathrm{kHz}$ \\
Ganho do modulador PWM & $K_{P W M}=0,0002$ \\
Frequência de cruzamento desejada & $\omega_{C}=2 \pi f_{S} / 27$ \\
Margem de fase desejada & $M F_{d s}=\phi_{d}=89^{\circ}$ \\
\hline
\end{tabular}

Fonte: Dados da pesquisa do autor. 
Considerando a Figura 5.7, que mostra o diagrama de blocos em malha aberta do conversor série, obtém-se a função de transferência da malha de corrente utilizada para o projeto do controlador, conforme equação (5.18). A função de transferência em malha aberta da planta compensada $G_{M A_{C S}}$ é obtida pela equação (5.21).

De acordo com os parâmetros da Tabela 5.1, e conforme as equações (5.58), (5.60), (5.62) e (5.63) são obtidos os ganhos do controlador P-RES do conversor série de corrente,

$$
\begin{gathered}
K p_{s}=400 \Omega, \\
k_{R_{1}}=k_{R 3}=k_{R 5}=k_{R 7}=1000 \Omega .
\end{gathered}
$$

A Figura 5.18 mostra a resposta em frequência de $G_{P_{C S}+R E S_{k}}, G_{M A} C S, G_{c S}$. Por meio do diagrama de bode, verifica-se que a magnitude da planta compensada na frequência desejada $\omega_{c}$ está em $0 \mathrm{~dB}$, bem como a margem de fase desejada é igual a 89ำ na frequência de $4650 \mathrm{rad} / \mathrm{s}$, comprovando que o controlador P-RES projetado atende as especificações de projeto.

Figura 5.18 - Resposta em frequência das funções $G_{P_{C S}+R E S_{k}}, G_{M A} C S, G_{C S}$

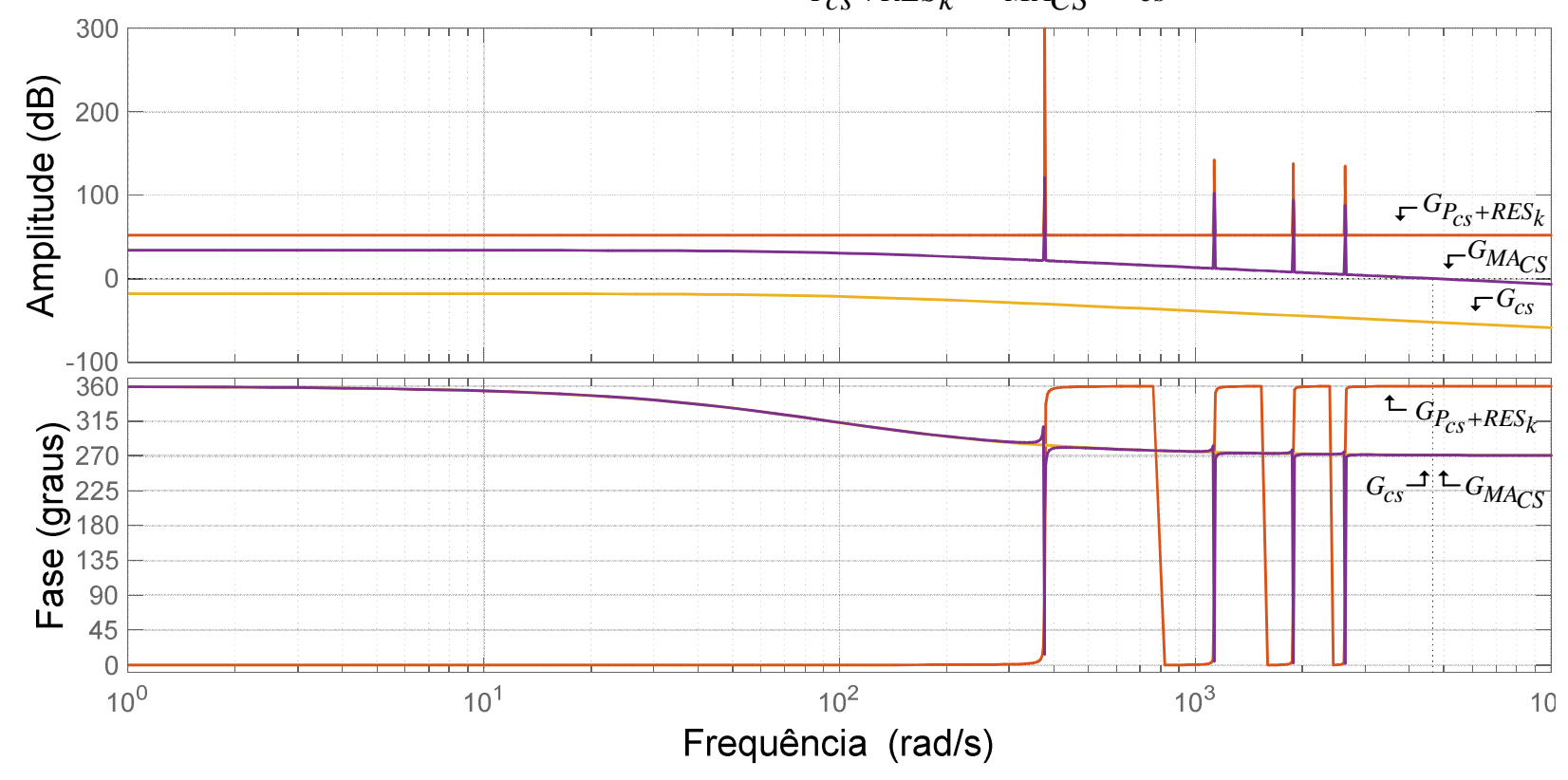

Fonte: Dados da pesquisa do autor.

A função de transferência da planta compensada em malha fechada é apresentada na equação (5.22). De forma a verificar a banda passante da malha de corrente do conversor multinível série de potência, com o controlador P-RES 
projetado, é mostrado o diagrama de bode em malha fechada na Figura 5.19. A faixa de passagem em -3dB é próxima de $4660 \mathrm{rad} / \mathrm{s}$, ou seja, aproximadamente $741,66 \mathrm{~Hz}$.

Figura 5.19 - Resposta em frequência em laço fechado da malha de corrente com controlador P-RES

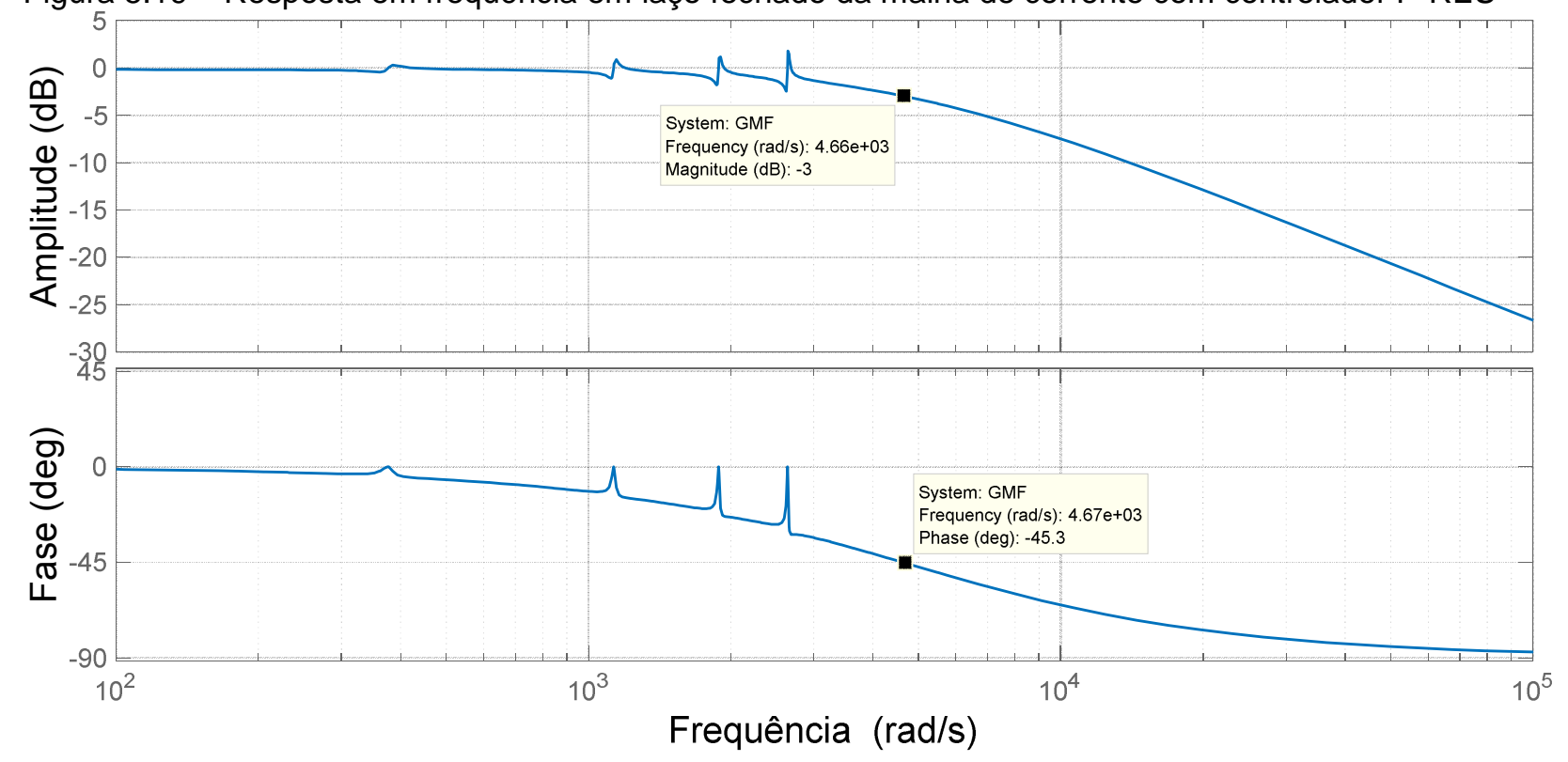

Fonte: Dados da pesquisa do autor.

$\mathrm{Na}$ Figura 5.20, são apresentados os polos da função de transferência em malha fechada do conversor série, de acordo com a equação (5.22), em que pode-se verificar que todos os polos estão localizados no semi-plano esquerdo do plano $s$, ou seja, o sistema é estável de acordo com os valores projetados do controlador P-RES. Já na Figura 5.21, são apresentados os mesmos polos, mas com maior ângulo de visão. 
Figura 5.20 - Mapa de polos da função de transferência de malha fechada da malha de corrente com controlador P-RES

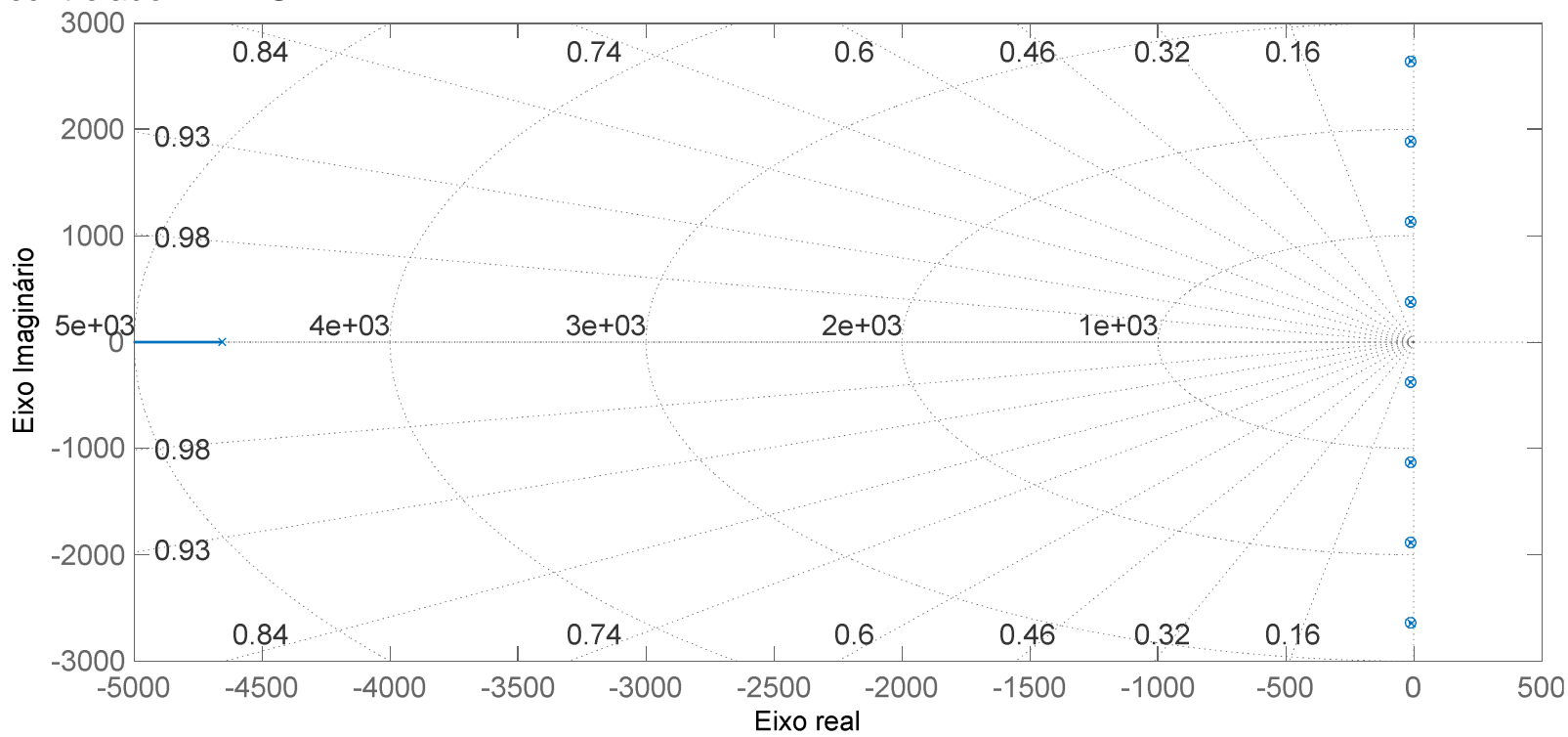

Fonte: Dados da pesquisa do autor.

Figura 5.21 - Mapa de polos e zeros da função de transferência de malha fechada da malha de corrente com controlador P-RES

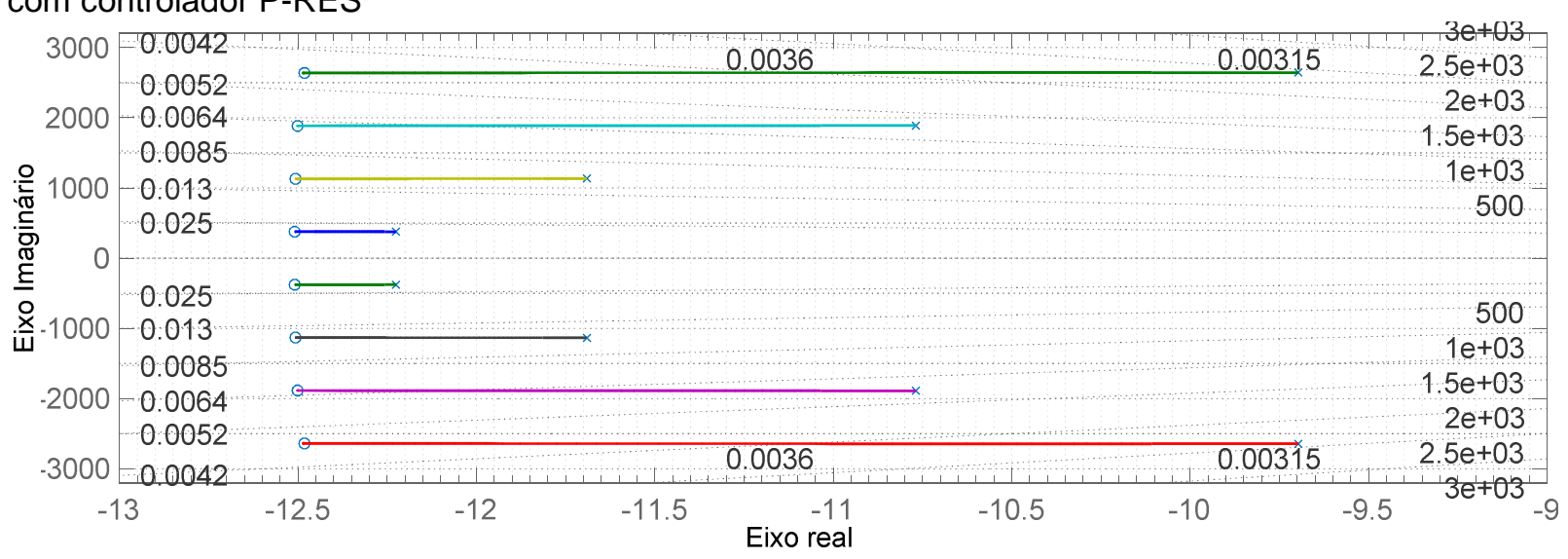

Fonte: Dados da pesquisa do autor.

\subsubsection{Projeto do controlador P-RES e P da malha de controle do conversor paralelo}

A tabela 5.2 apresenta as especificações adotadas para o projeto do controlador P-RES e para o controlador proporcional, utilizado nas malhas de corrente e de tensão do conversor paralelo, modelado na Seção 5.5. De acordo com a modelagem apresentada, têm-se duas malhas de controle utilizadas no conversor paralelo de tensão, sendo uma malha interna de corrente, que utiliza um controlador 
proporcional, e uma malha externa de tensão que é composta por um controlador PRES.

De modo a obter os ganhos $K p_{i}, K p_{v}, R E S_{k}$ dos controladores referentes às equações (5.29) e (5.30), foram utilizadas as metodologias conforme os itens 5.11.1 e 5.11.2, as funções de transferência em malha aberta, o diagrama de bode para mostrar a resposta em frequência dos controladores, bem como os polos do sistema.

Tabela 5.2 - Especificações para projeto do controlador P de corrente e P-RES de tensão do conversor paralelo.

\begin{tabular}{ll}
\hline Indutância de acoplamento do conversor paralelo & $L_{f p}=1,3 \mathrm{mH}$ \\
Resistência série do indutor de acoplamento do conversor paralelo & $R_{L_{f p}}=0,21 \Omega$ \\
Capacitância de filtragem & $C_{f p}=80 \mu \mathrm{F}$ \\
Tensão do elo CC total & $V_{c c_{t o t a l}}=250 \mathrm{~V}$ \\
Frequência de chaveamento do conversor de potência paralelo & $f_{S}=20 \mathrm{kHz}$ \\
Ganho do modulador PWM & $K_{P W M}=0,0002$ \\
Frequência de cruzamento desejada da malha interna de corrente & $\omega_{c P i}=2 \pi f_{s} / 4$ \\
Frequência de cruzamento desejada da malha externa de tensão & $\omega_{c P v}=2 \pi f_{s} / 33$ \\
Margem de fase desejada da malha externa de tensão & $M F_{d v p}=\phi_{d_{v p}}=86^{\circ}$ \\
\hline
\end{tabular}

Fonte: Dados da pesquisa do autor.

Com a função de transferência de laço aberto da malha interna de corrente $G_{M A_{i}}(s)$, apresentada na equação (5.32), e com os parâmetros da Tabela 5.2, bem como as equações (5.58) e (5.60), obtém-se o ganho do controlador proporcional da malha interna de corrente do conversor paralelo,

$$
K p_{i}=320 \Omega \text {. }
$$

A Figura 5.22 mostra a resposta em frequência de $G_{c p i}, G_{M A_{i}}, G_{M F_{i}}$. Por meio do diagrama de bode, verifica-se que a magnitude da planta compensada na frequência desejada $\omega_{c}$ está em $0 \mathrm{~dB}$, em $3240 \mathrm{rad} / \mathrm{s}$. Entretanto, a margem de fase da mesma não sofre variações por causa do controlador proporcional na malha de controle interna de corrente do conversor paralelo. 
Figura 5.22 - Resposta em frequência das funções $G_{c p i}, G_{M A_{i}}, G_{M F_{i}}$

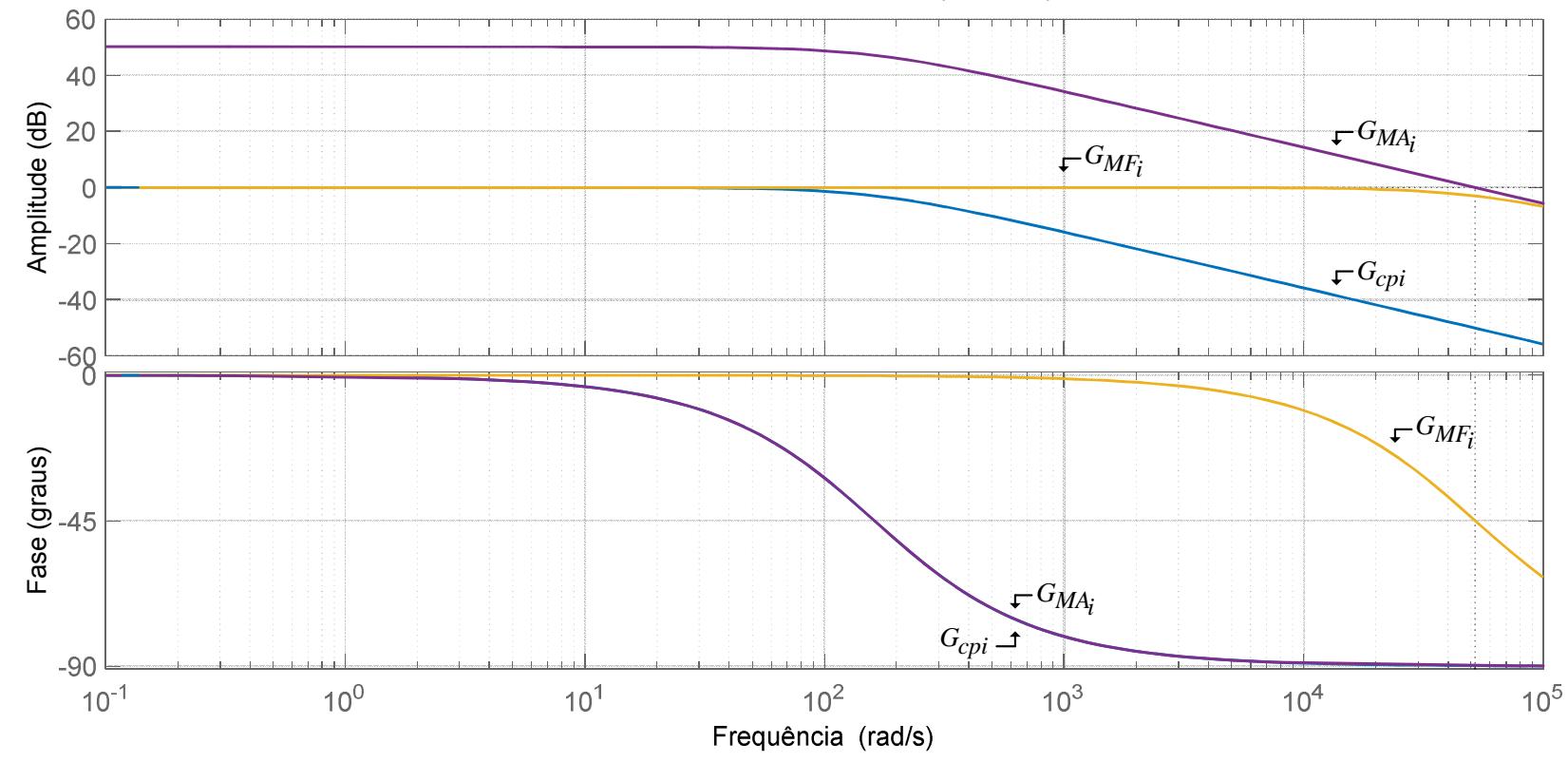

Fonte: Dados da pesquisa do autor.

Assim, de acordo com os parâmetros da Tabela 5.2, são obtidos os ganhos do controlador proporcional ressonante da malha externa de tensão do conversor paralelo,

$$
\begin{gathered}
K p_{v}=0,11 \Omega, \\
k_{R_{1}}=k_{R_{3}}=k_{R_{5}}=k_{R_{7}}=50 \Omega .
\end{gathered}
$$

A Figura 5.23 mostra a resposta em frequência de $G_{P_{c p}}+R E S_{k}, G_{M A_{v}}, G_{M A_{c v}}$. Por meio do diagrama de bode, verifica-se que a magnitude da planta compensada na frequência desejada $\omega_{c}$ está em $0 \mathrm{~dB}$, bem como a margem de fase desejada é igual a $86^{\circ}$ na frequência de $3850 \mathrm{rad} / \mathrm{s}$, comprovando que o controlador P-RES projetado atende as especificações de projeto. 
Figura 5.23 - Resposta em frequência das funções $G_{P_{c p}}+R E S_{k}, G_{M A_{v}}, G_{M A_{c v}}$

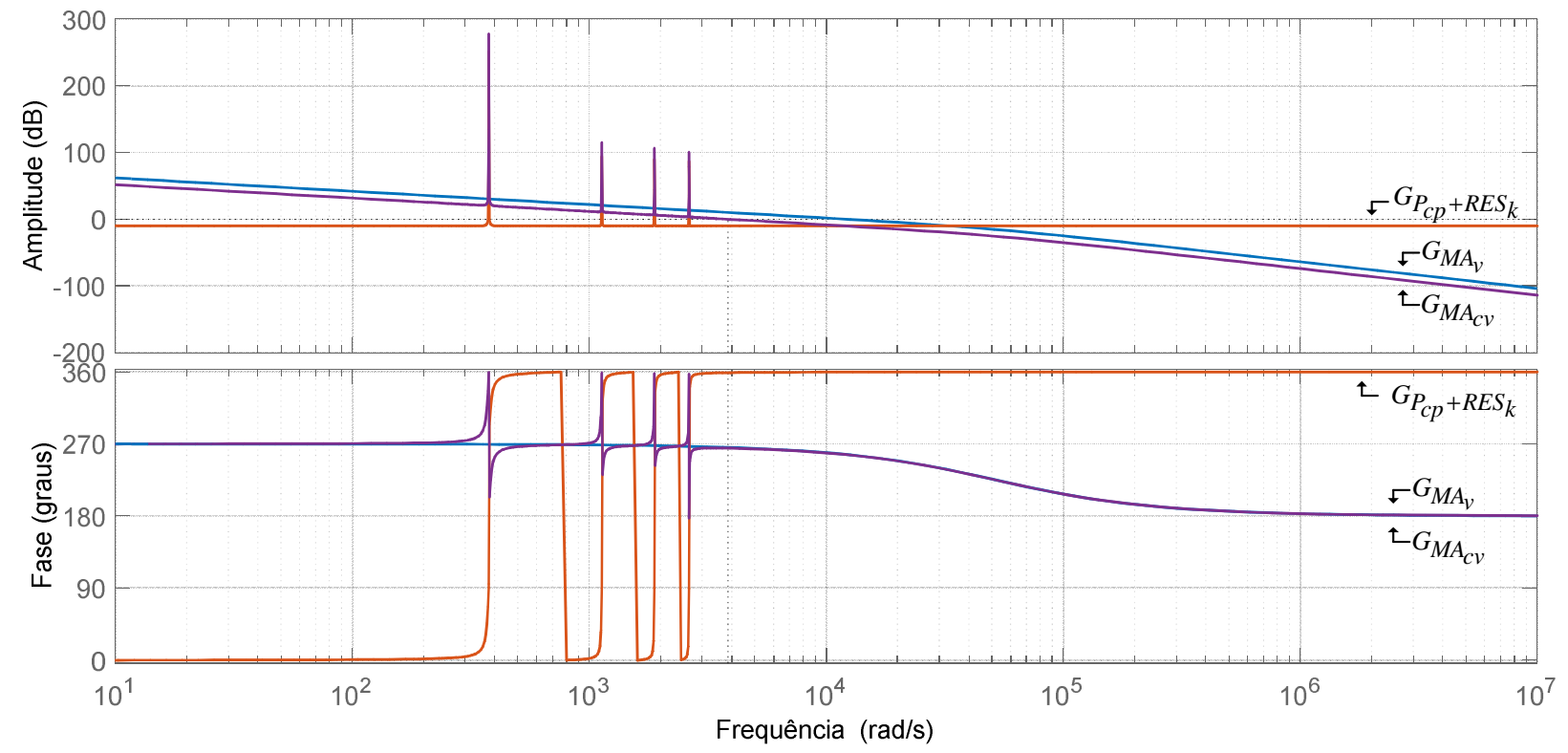

Fonte: Dados da pesquisa do autor.

A função de transferência da planta compensada em malha fechada é apresentada na equação (5.36). De forma a verificar a banda passante da malha de tensão do conversor multinível paralelo de potência, com os controladores P-RES e $P$ projetado, é mostrado o diagrama de bode em malha fechada na Figura 5.24. A faixa de passagem em -3dB é próxima de $4230 \mathrm{rad} / \mathrm{s}$, ou seja, aproximadamente $673,22 \mathrm{~Hz}$.

Figura 5.24 - Resposta em frequência em laço fechado da malha de tensão com controlador P-RES e $\mathrm{P}$

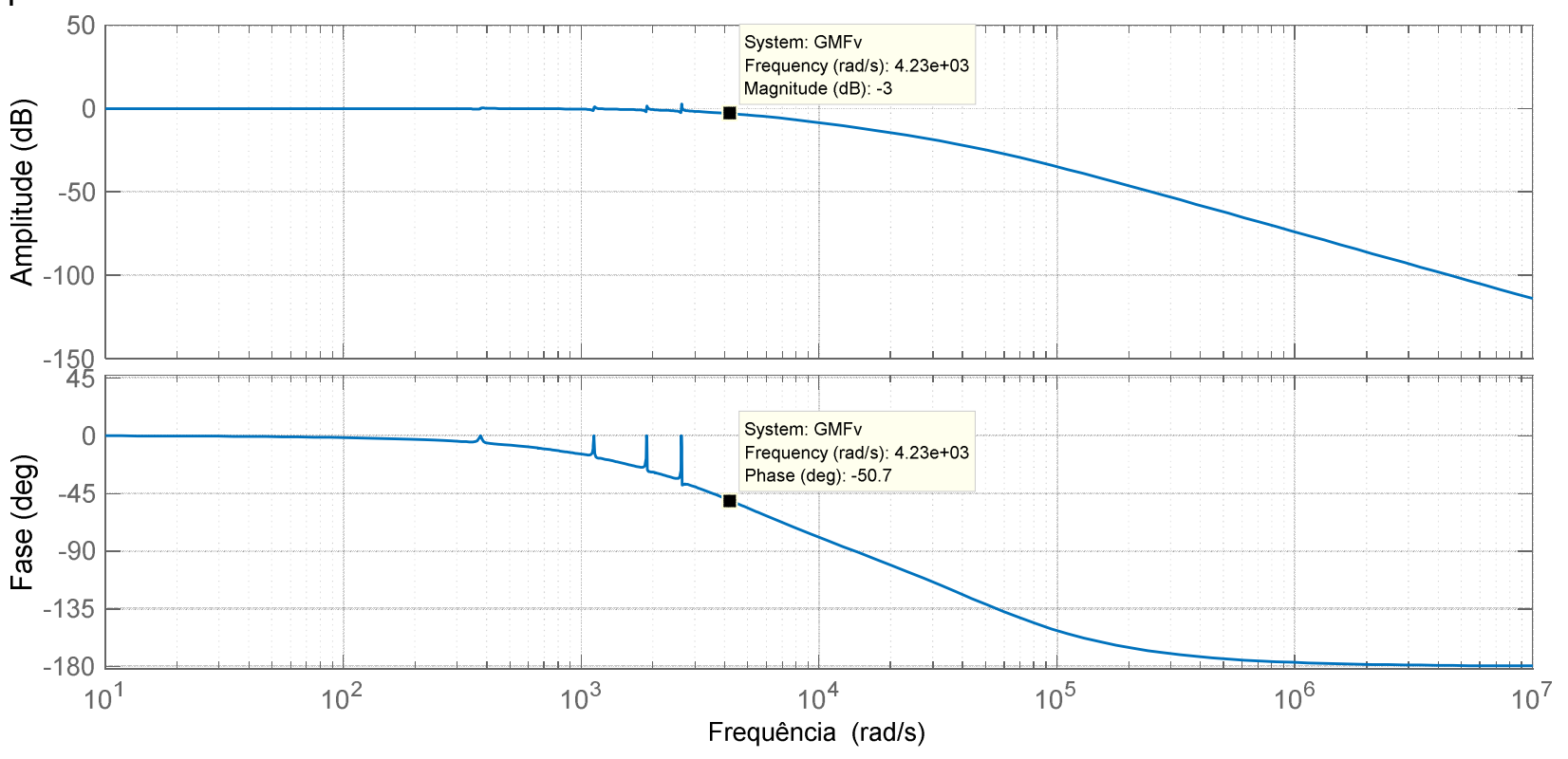

Fonte: Dados da pesquisa do autor. 
Na Figura 5.25, são apresentados os polos da função de transferência em malha fechada do conversor paralelo, de acordo com a equação (5.36), em que podese verificar que todos os polos estão localizados no semi-plano esquerdo do plano $s$, ou seja, o sistema é estável de acordo com os valores projetados do controlador PRES e $P$.

Figura 5.25 - Mapa de polos e zeros da função de transferência em laço fechado da malha de tensão com controlador P-RES e $P$

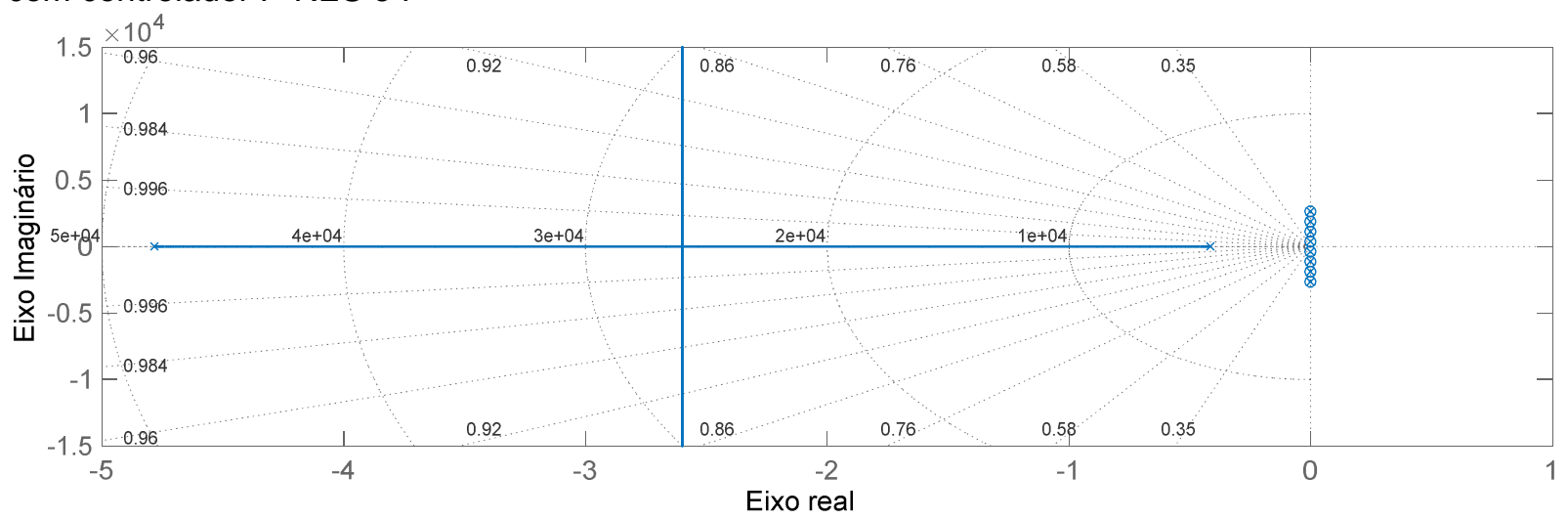

Fonte: Dados da pesquisa do autor.

\subsubsection{Projeto do controlador PI da malha de controle do elo CC}

A tabela 5.3 apresenta as especificações adotadas para o projeto do controlador PI, utilizado nas malhas de tensão do elo CC total e do desequilibrado, modelado nas seções 5.7 e 5.9 .

Tabela 5.3 - Especificações para projeto do controlador PI de tensão do elo CC.

\begin{tabular}{ll}
\hline Tensão do elo CC total & $V_{c c_{\text {total }}}=250 \mathrm{~V}$ \\
Ondulação do elo CC & $f_{S}=120 \mathrm{~Hz}$ \\
Capacitância do elo CC & $C_{c c}=4700 \mu \mathrm{F}$ \\
Frequência de cruzamento desejada da malha de tensão do elo CC & $\omega_{c c}=2 \pi f_{S} / 40$ \\
$\begin{array}{l}\text { Frequência de cruzamento desejada da malha de desequilíbrio de tensão } \\
\text { do elo CC }\end{array}$ & $\omega_{c c_{d}}=2 \pi f_{S} / 80$ \\
Margem de fase desejada da malha de tensão do elo CC & $M F_{c c}=\phi_{c c}=89^{\circ}$ \\
\hline
\end{tabular}

Fonte: Dados da pesquisa do autor. 
De modo a obter os ganhos $K p_{v c c}, K i_{v c c}, K p_{v c c d}, K i_{v c c d}$ controladores referentes às equações (5.47) e (5.54), foi utilizada a metodologia conforme o item 5.11.1, as funções de transferência em malha aberta e o diagrama de bode para mostrar a resposta em frequência dos controladores.

De acordo com os parâmetros da Tabela 5.3, e conforme as equações (5.58), (5.60), são obtidos os ganhos do controlador PI de tensão do elo CC total e desequilibrado,

$$
\begin{gathered}
K p_{v c c}=0,3515 \Omega, K i_{v c c}=0,1157 \Omega / s, \\
K p_{v c c d}=0,0879 \Omega, K i_{v c c d}=0,0072 \Omega / s .
\end{gathered}
$$

De forma a verificar a banda passante da malha de tensão do elo CC, com os controladores PI projetados, é mostrado o diagrama de bode em malha fechada na Figura 5.26. A faixa de passagem da tensão do elo CC em -3dB é de $19 \mathrm{rad} / \mathrm{s}$, e da tensão de desequilíbrio em -3dB é de 4,74 rad/s.

Figura 5.26 - Resposta em frequência em laço fechado da malha de tensão do elo CC com controlador $\mathrm{PI}$

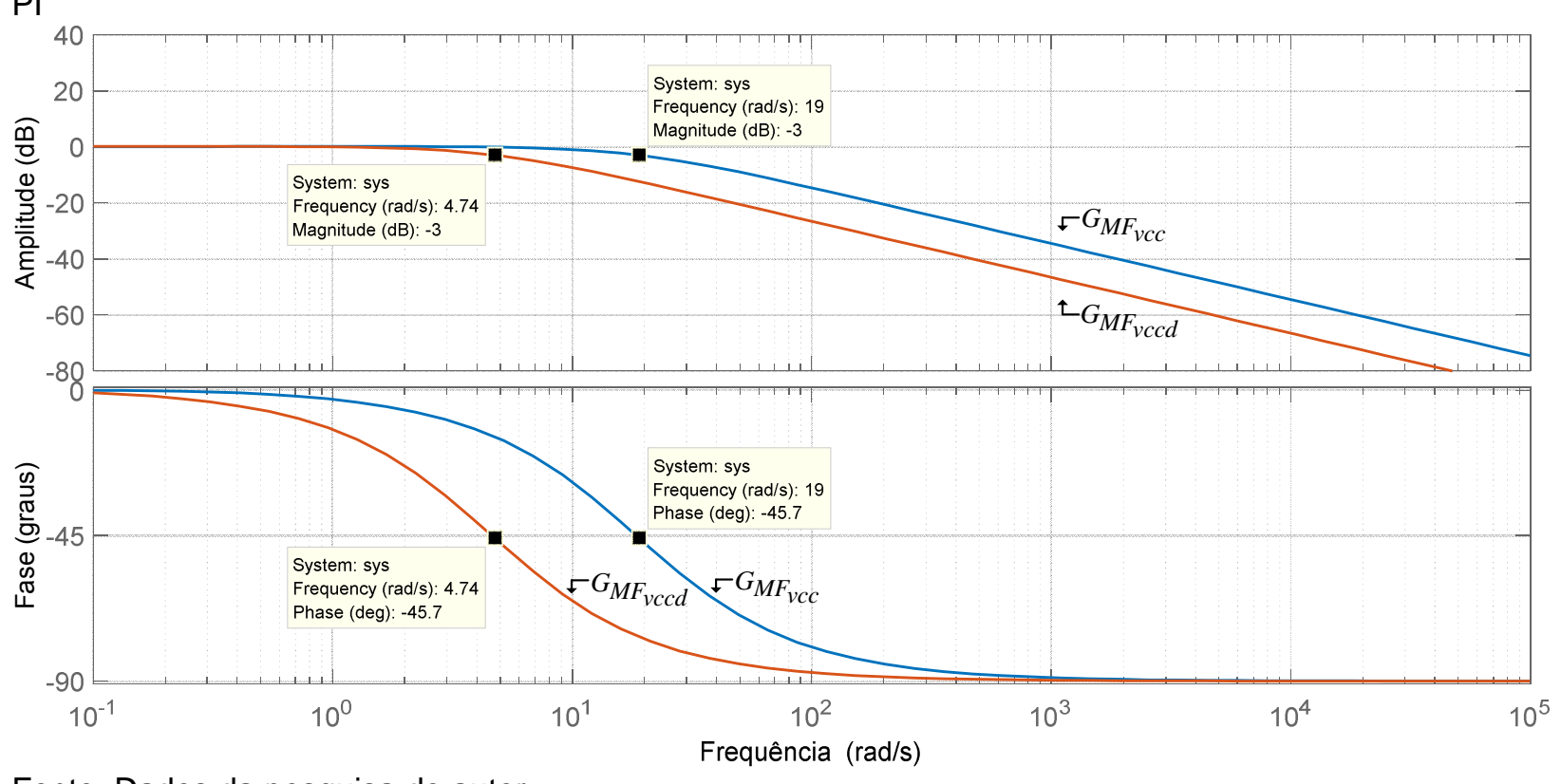

Fonte: Dados da pesquisa do autor. 


\subsection{Fluxo de Potência do UPQC}

Na estrutura do UPQC, quando em operação, ocorre um fluxo de potência, ou seja, um fluxo de potência por meio dos conversores bidirecionais série e paralelo, que estão interligados por um elo CC, a partir da rede elétrica para a carga, ou viceversa, de acordo com suas características, bem como da energia armazenada no elo CC.

As potências aparentes processadas por meio dos conversores série $S_{S}$ e paralelo $S_{p}$ dependem da relação entre os valores eficazes das tensões de entrada $V_{s}$ e de saída $V_{L}$ do UPQC, ou seja, $\left(V_{s} / V_{L}\right)$, da TDH da corrente de carga $i_{L}\left(T D H_{i_{L}}\right)$ , do fator de deslocamento $\cos \varphi_{1}$, e do fator de carga do elo CC $k_{c c}$, que é a relação entre a potência ativa do elo CC $P_{c c}$ e a potência ativa consumida na carga $P_{L}$, ou seja, $\left(k_{c c}=P_{c c} / P_{L}\right)$ (Silva et al, 2002).

Admitindo-se que as tensões de entrada e de saída são grandezas senoidais, assim como a corrente de entrada do UPQC, em regime permanente, as potências aparentes dos conversores multiníveis série e paralelo, normalizadas em função da potência aparente da carga, são (Silva et al, 2002),

$$
\begin{gathered}
\left|\frac{S_{S}}{S_{L}}\right|=\frac{\cos \varphi_{1} \sqrt{\left[\left(1+k_{c c}\right)\left(1-V_{L} / V_{S}\right)\right]^{2}}}{\sqrt{1+T D H_{i_{L}}^{2}}}, \\
\left|\frac{S_{p}}{S_{L}}\right|=\sqrt{\frac{\cos ^{2} \varphi_{1} \frac{V_{L}}{V_{S}}\left(1+k_{c c}\right)\left(\frac{V_{L}}{V_{S}}\left(1+k_{c c}\right)-2\right)}{\sqrt{1+T D H_{i_{L}}^{2}}}}+1 .
\end{gathered}
$$

Nas figuras 5.27 a 5.28, são apresentadas as curvas das potências aparentes dos conversores bidirecionais série e paralelo, normalizados em função da potência aparente total da carga, de acordo com as equações descritas em (5.71) e em (5.72), ou seja, $\left|S_{s} / S_{L}\right|$ e $\left|S_{p} / S_{L}\right|$. 
A Figura 5.27 mostra as curvas normalizadas de potência, considerando os fatores $k_{c c}=0, k_{c c}=0,1, \cos \varphi=0,7$ e $\cos \varphi=1$, para o conversor série. Pode-se observar que o processamento de potência depende da relação entre $V_{S} / V_{L}$ quando for diferente de um, para $T D H_{i_{L}}=0 \%$. No entanto, para $T D H_{i_{L}}>0 \%$, a potência processada aumenta na faixa que contém a relação $V_{s} / V_{L}$, ou seja, $0,75<V_{s} / V_{L}<1,25$. Quando tem-se $k_{c c}=0,1$, existe uma potência ativa drenada da rede elétrica para manter regulada a tensão do elo CC, de forma a compensar as perdas nos conversores e elementos passivos. Neste caso, existe um aumento na potência processada pelo conversor série.

Na Figura 5.28, são apresentadas as curvas normalizadas de potência para o conversor paralelo, considerando os fatores $k_{c c}=0, k_{c c}=0,1, \cos \varphi=0,7$ e $\cos \varphi=1$. Verifica-se que quanto maior a $T D H_{i_{L}}$ e menor o $\cos \varphi$, maior será a potência processada no conversor. Quando tem-se $k_{c c}=0,1$, verifica-se também que há um aumento na potência processada pelo conversor, de acordo com as condições, ou seja, variações de $V_{s} / V_{L}, T D H_{i_{L}}$ e $\cos \varphi$.

Figura 5.27 - Curvas das potências aparentes normalizadas do conversor série $\left|S_{S} / S_{L}\right|$.

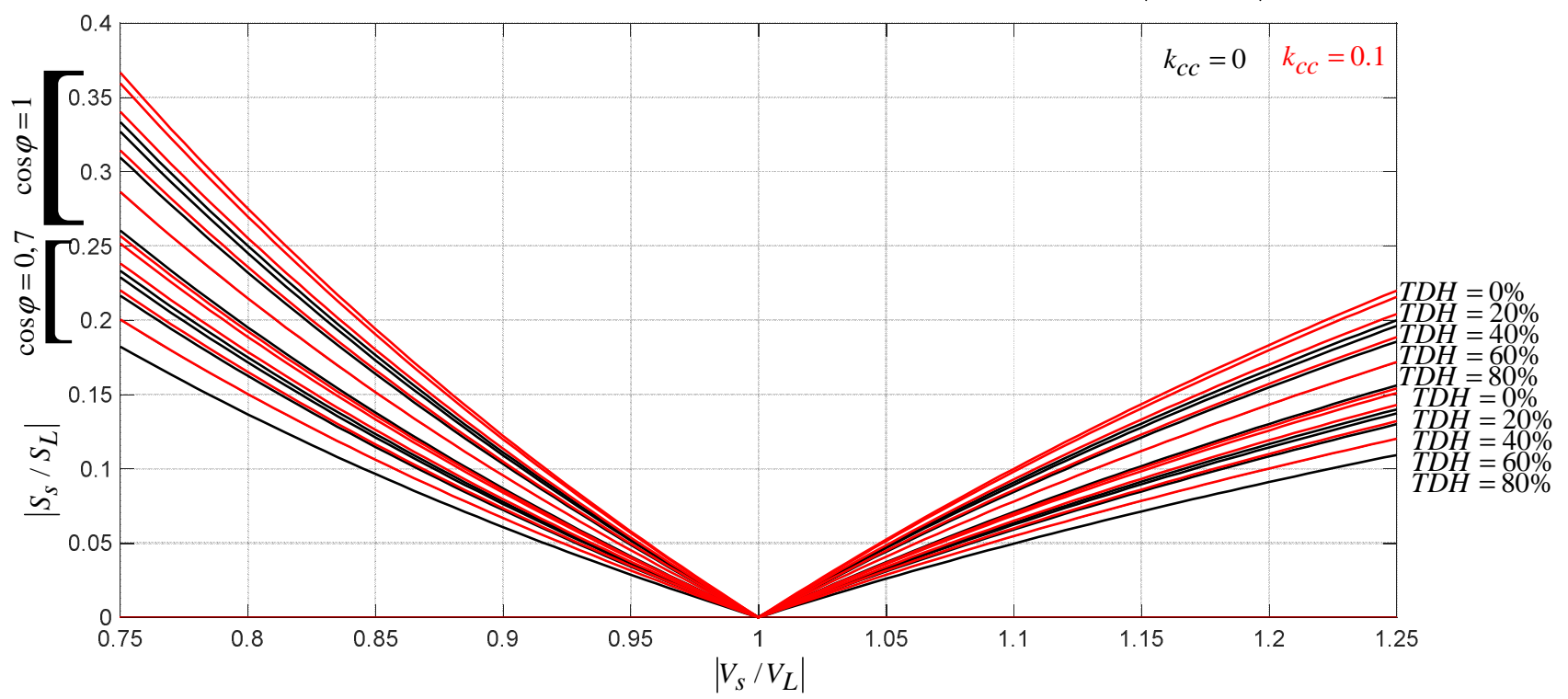

Fonte: Dados da pesquisa do autor. 
Figura 5.28 - Curvas das potências aparentes normalizadas do conversor paralelo $\left|S_{p} / S_{L}\right|$.

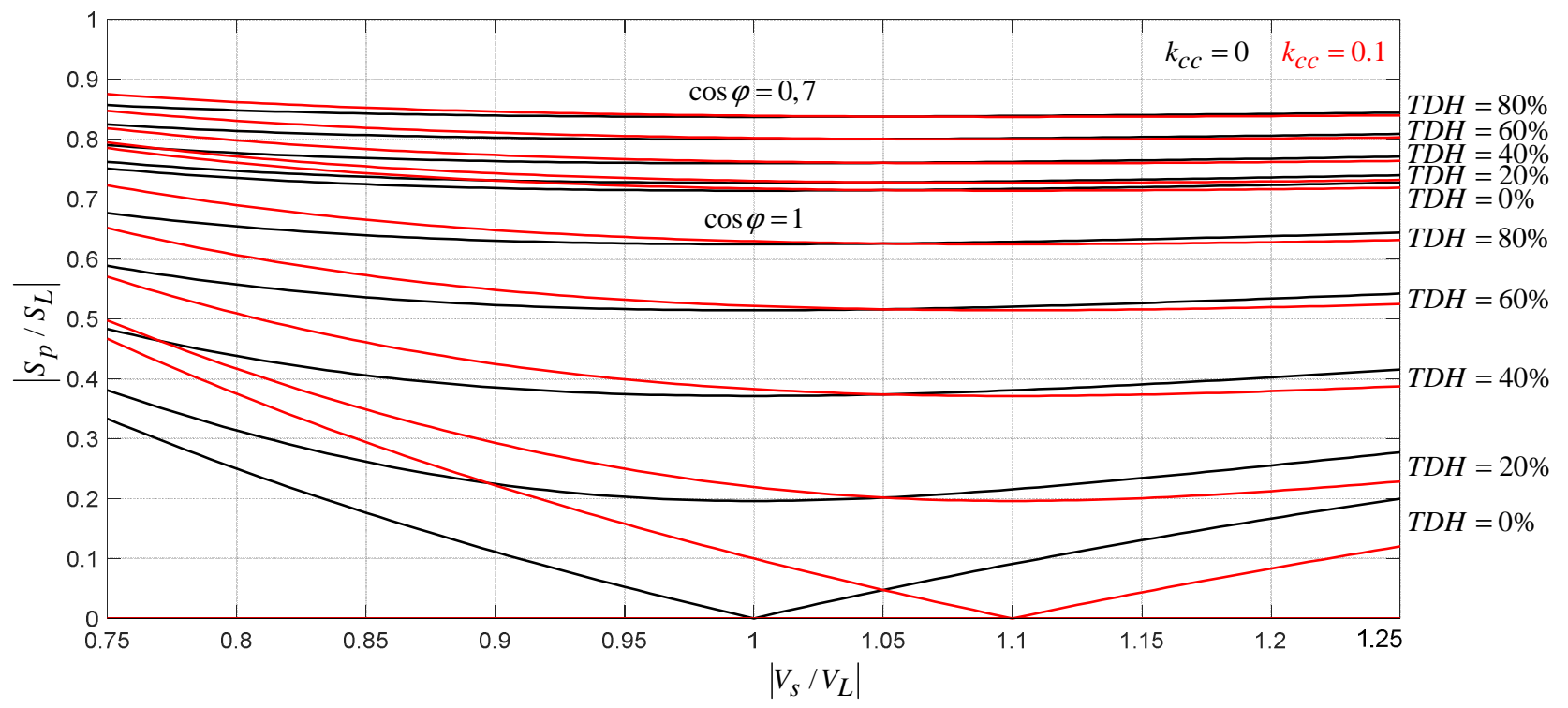

Fonte: Dados da pesquisa do autor.

O sentido do fluxo de potência pode mudar nos conversores série e paralelo, dependendo da tensão de entrada $V_{S}$ em relação a tensão de saída $V_{L}$. As figuras 5.29, 5.30, 5.31 e 5.32 mostram o fluxo das potências ativas instantâneas no UPQC, de modo que a potência da rede elétrica é $p_{s}$, do conversor série é $p_{c s}$, do conversor paralelo é $p_{c p}$ e da carga é $p_{L}$, respectivamente, e $p_{o}=p_{L}-p_{c p}=p_{s}-p_{c s}$.

Considerando $k_{c c}=0$, quando $V_{s}$ é maior que $V_{L}$, o fluxo de potência ativa flui da rede elétrica para o elo CC via conversor série, e do elo CC para a carga via conversor paralelo, ou seja, $p_{s}=p_{c s}+p_{o}=p_{c p}+p_{o}=p_{L}$, conforme Figura 5.29.

Figura 5.29 - Fluxo de potência: $V_{s}>V_{L}$ e $k_{c c}=0$.

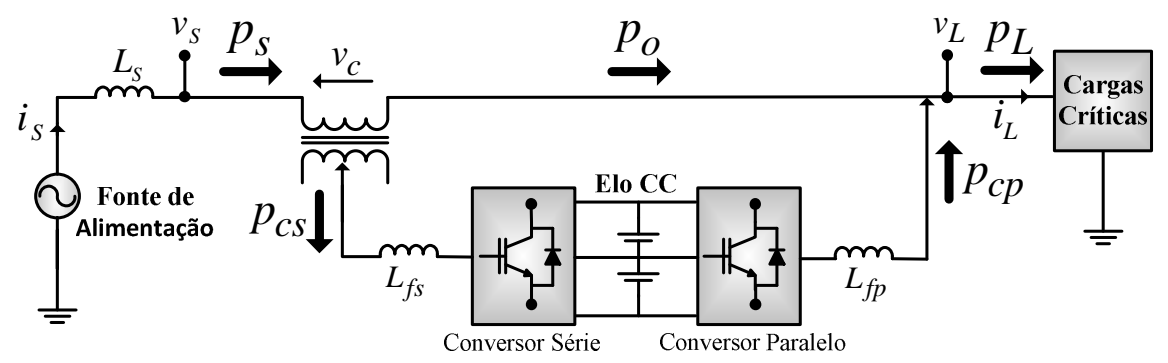

Fonte: Dados da pesquisa do autor.

Quando $V_{S}$ é menor que $V_{L}$, o controlador de tensão do elo CC eleva a corrente de entrada, de forma a equilibrar o fluxo de potência. O fluxo de potência ativa flui da 
carga para o elo CC via conversor paralelo, e do elo CC para a rede elétrica via conversor série e transformador de acoplamento, ou seja, $p_{s}+p_{c s}=p_{o}=p_{c p}+p_{L}$, sendo que $p_{c s}=p_{c p}$, conforme Figura 5.30.

Figura 5.30 - Fluxo de potência: $V_{s}<V_{L}$ e $k_{c c}=0$.

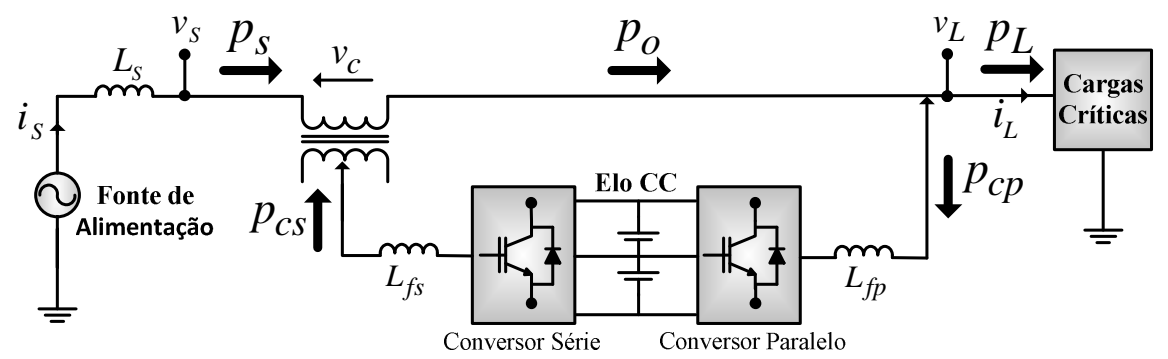

Fonte: Dados da pesquisa do autor.

Considerando a tensão $V_{s}$ igual a $V_{L}$, e o UPQC alimentando uma carga puramente resistiva, não existe fluxo de potência ativa circulando por meio dos conversores série e paralelo, ou seja, $p_{S}=p_{o}=p_{L}$, como mostra a Figura 5.31.

Figura 5.31 - Fluxo de potência: $V_{s}=V_{L}$ e $k_{c c}=0$.

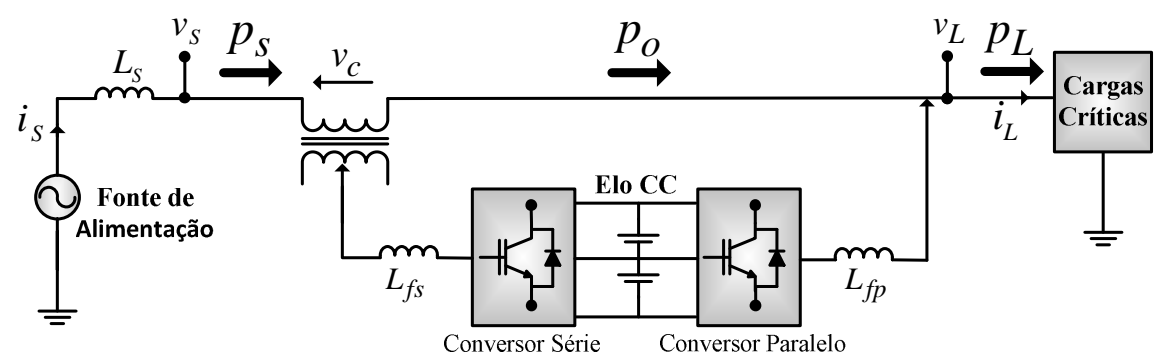

Fonte: Dados da pesquisa do autor.

No entanto, a potência ativa relacionada à carga do elo $\mathrm{CC}$, às perdas de comutação dos conversores, bem como aos elementos passivos, é absorvida pelo conversor paralelo. Com base nas figuras 5.27 e 5.28, para $V_{S} / V_{L}=1, \cos \varphi=1$, $T D H_{i_{L}}=0$, e $k_{c c}=0.1$, as potências normalizadas $\left|S_{p} / S_{L}\right|=0.1$ e $\left|S_{s} / S_{L}\right|=0$, é mostrado na Figura 5.32, o fluxo de potência do UPQC sob esta condição. 
Figura 5.32 - Fluxo de potência: $V_{s}=V_{L}$ e $k_{c c}=0.1$.

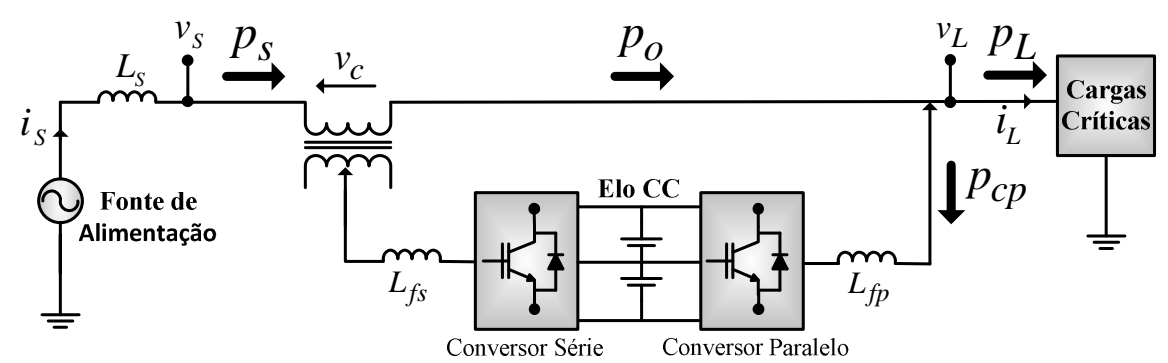

Fonte: Dados da pesquisa do autor.

A partir da análise do fluxo de potência do UPQC, é possível dimensionar os conversores série e paralelo. Assim, o conversor paralelo de tensão deve ser dimensionado de acordo com a potência nominal da carga. Já o conversor série de corrente deve ser considerado para o dimensionamento, a compensação de distúrbios presente na tensão da rede elétrica como, harmônicos, elevações e afundamentos de tensão.

Quando ocorrer esses distúrbios, uma componente fundamental de tensão surge sobre os terminais do transformador de acoplamento série, que corresponde à diferença entre as componentes fundamentais das tensões de entrada e de saída do UPQC. No mesmo momento, tem-se uma componente fundamental da corrente compensada da rede elétrica no transformador de acoplamento, assim, resultando em uma potência média que será absorvida ou fornecida pelo conversor série de corrente.

\subsection{Análise de estabilidade do UPQC}

Os conversores série e paralelo, são analisados mediante a ocorrência de distúrbios na corrente da carga e na tensão da rede, de modo a verificar a capacidade de estabilidade do sistema. É utilizado o critério de estabilidade de Routh-Hurwitz, que define que todos os coeficientes do polinômio denominador analisado em suas respectivas funções de transferência em malha fechada tenham o mesmo sinal para a planta ser sempre estável.

A análise de estabilidade do conversor série de potência é realizada por meio da função de transferência em malha fechada apresentada em (5.22), tendo todos os coeficientes do polinômio denominador positivos, referente a Figura 5.6, para o caso de variação de carga. Neste caso, o conversor série sempre opera como fonte de 
corrente senoidal, as variações de carga interferem somente na amplitude da referência de corrente da malha de controle, assim não influencia a estabilidade da mesma.

Já para o conversor paralelo de potência, apresentado na Figura 5.10, com a função de transferência em (5.36), não é possível analisar as variações de carga, se interferem na estabilidade da malha de controle da tensão de saída. Desse modo, é realizada uma mudança a partir do diagrama de blocos da Figura 5.10, que considera a corrente da carga $i_{L}$ como entrada e a tensão da carga $v_{L}$ como saída da planta, apresentado na Figura 5.33, respectivamente.

Assim, a corrente da rede $i_{s}$ é obtida por meio da indutância da rede $L_{r d}$ e dispersão do transformador série $L_{d}$, sendo a indutância equivalente representada por $L_{e q}=L_{r d}+L_{d}$, bem como a resistência série $R_{r d}, R_{d}$ de ambos, em que a resistência equivalente é dada por $R_{e q}=R_{r d}+R_{d}$. A função de transferência em malha fechada que representa a Figura 5.33 é dada por (5.73) (Modesto et al, 2016).

Figura 5.33 - Diagrama de blocos das malhas de controle do conversor paralelo para análise de estabilidade sob variações de carga

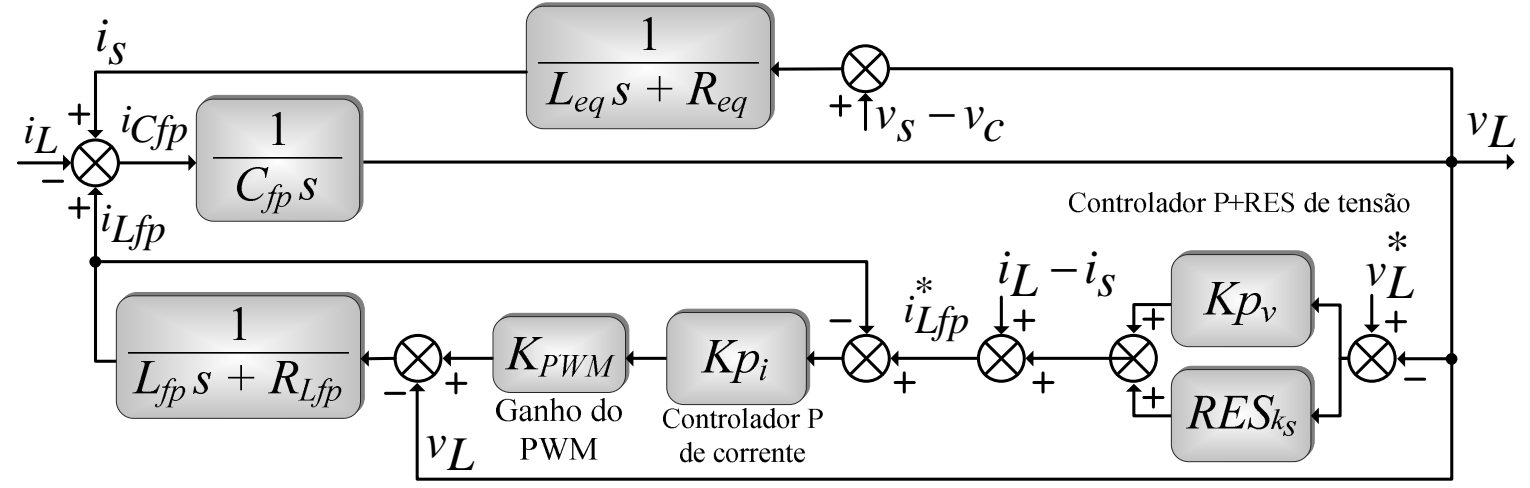

Fonte: Dados da pesquisa do autor.

$$
G_{M F_{P_{v i}}}=\frac{v_{L}}{i_{L}}=\frac{A_{1} s^{2}+A_{2} s+A_{3}}{B_{1} s^{3}+B_{2} s^{2}+B_{3} s+B_{4}},
$$

têm-se:

$$
A_{1}=-L_{e q} L_{f p} ; A_{2}=-L_{e q} R_{L f p}-L_{e q} K-L_{f p} R_{e q} ; A_{3}=-R_{e q} R_{L f p}-R_{e q} K ; K=K p_{i} K_{p w m},
$$




$$
\begin{gathered}
B_{1}=C_{p} L_{e q} L_{f p} ; B_{2}=C_{p} L_{e q} K+L_{e q} R E S_{k} K+C_{p} L_{f p} R_{e q}+C_{p} L_{e q} R_{L f p}, \\
B_{3}=C_{p} K R_{e q}+C_{p} R_{e q} R_{L f p}+L_{e q} K p_{v} K+R E S_{k} K R_{e q}+L_{f p} ; B_{4}=R_{e q} K p_{v} K+R_{L f p}+K .
\end{gathered}
$$

A partir do critério de Routh-Hurwitz, a equação (5.73) tem todos os coeficientes do polinômio denominador positivo, e as desigualdades $B_{2} B_{1}>B_{4} B_{3}$ devem ser respeitadas. Portanto, para ter essas condições, os parâmetros dos controladores $\mathrm{P}+\mathrm{RES}$ e $\mathrm{P}$, ou seja, $K p_{v}, R E S_{k}, K p_{i}$, devem ser ajustados. Assim, com os ganhos adquiridos em projeto, pode-se confirmar que a planta será estável mesmo com variações de carga.

Nas figuras 5.34 e 5.35, são apresentados os polos da função de transferência em relação à equação (5.73), para valores diferentes de $L_{e q}$ e $R_{e q}$. Na Figura 5.34, considera-se $R_{e q}=0.5 \Omega$, e varia-se $L_{e q}$.

Figura 5.34 - Mapa de polos da FT em malha fechada sob análise de estabilidade do conversor paralelo $\left(1 \mu H \leq L_{e q} \leq 2000 \mu H\right)$ e $\left(R_{e q}=0.5 \Omega\right)$.

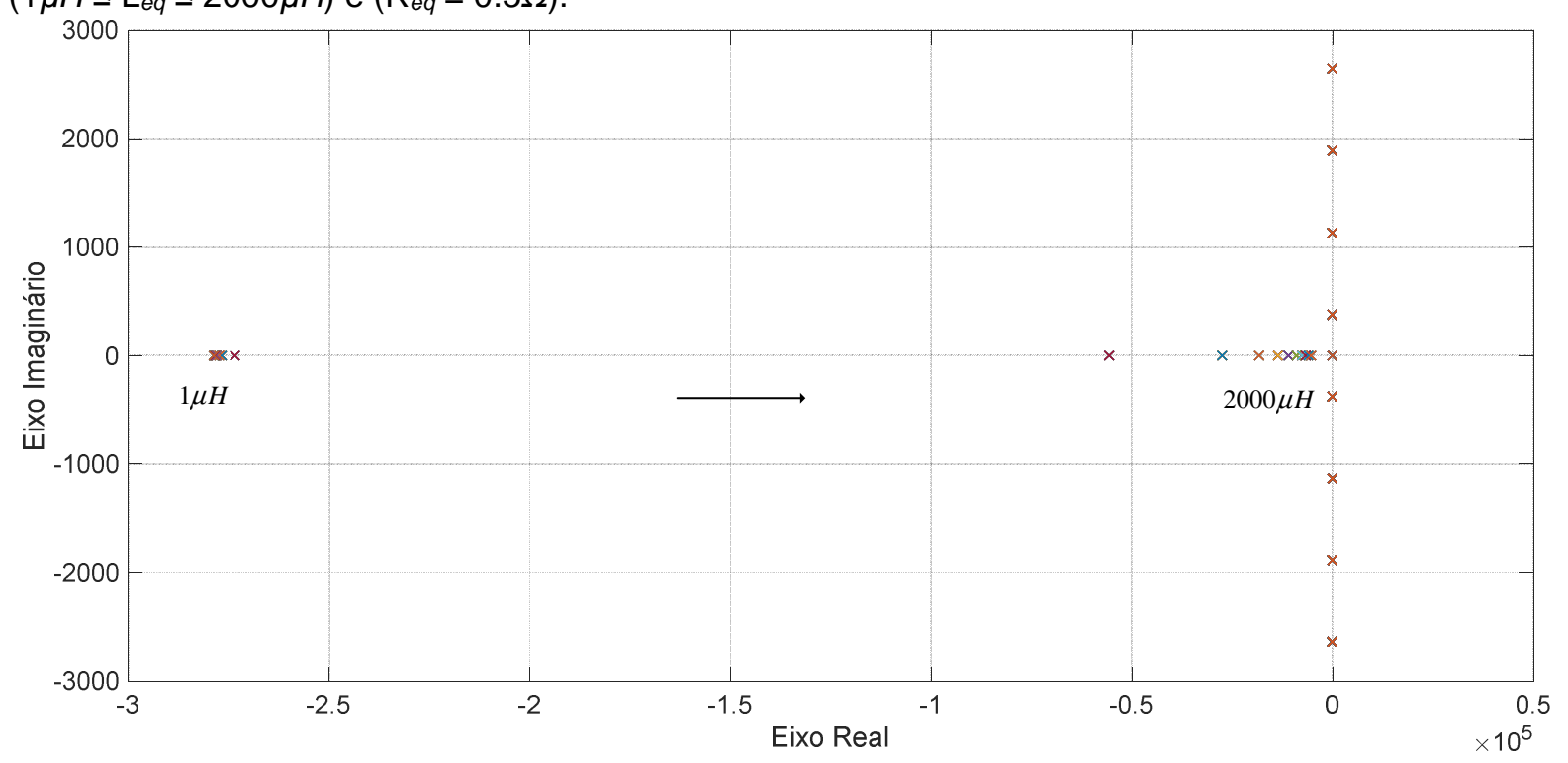

Fonte: Dados da pesquisa do autor.

Já na Figura 5.35, considera-se $L_{e q}=10 \mu \mathrm{H}$, e varia-se $R_{e q}$. Assim, são observados que todos os polos estão localizados no semi-plano esquerdo do plano $s$. A estabilidade do sistema não é alterada sob características diferentes da impedância da rede elétrica. 
Figura 5.35 - Mapa de polos da FT em malha fechada sob análise de estabilidade do conversor paralelo $\left(0.1 \Omega \leq R_{e q} \leq 100 \Omega\right)$ e $\left(R_{e q}=10 \mu H\right)$.

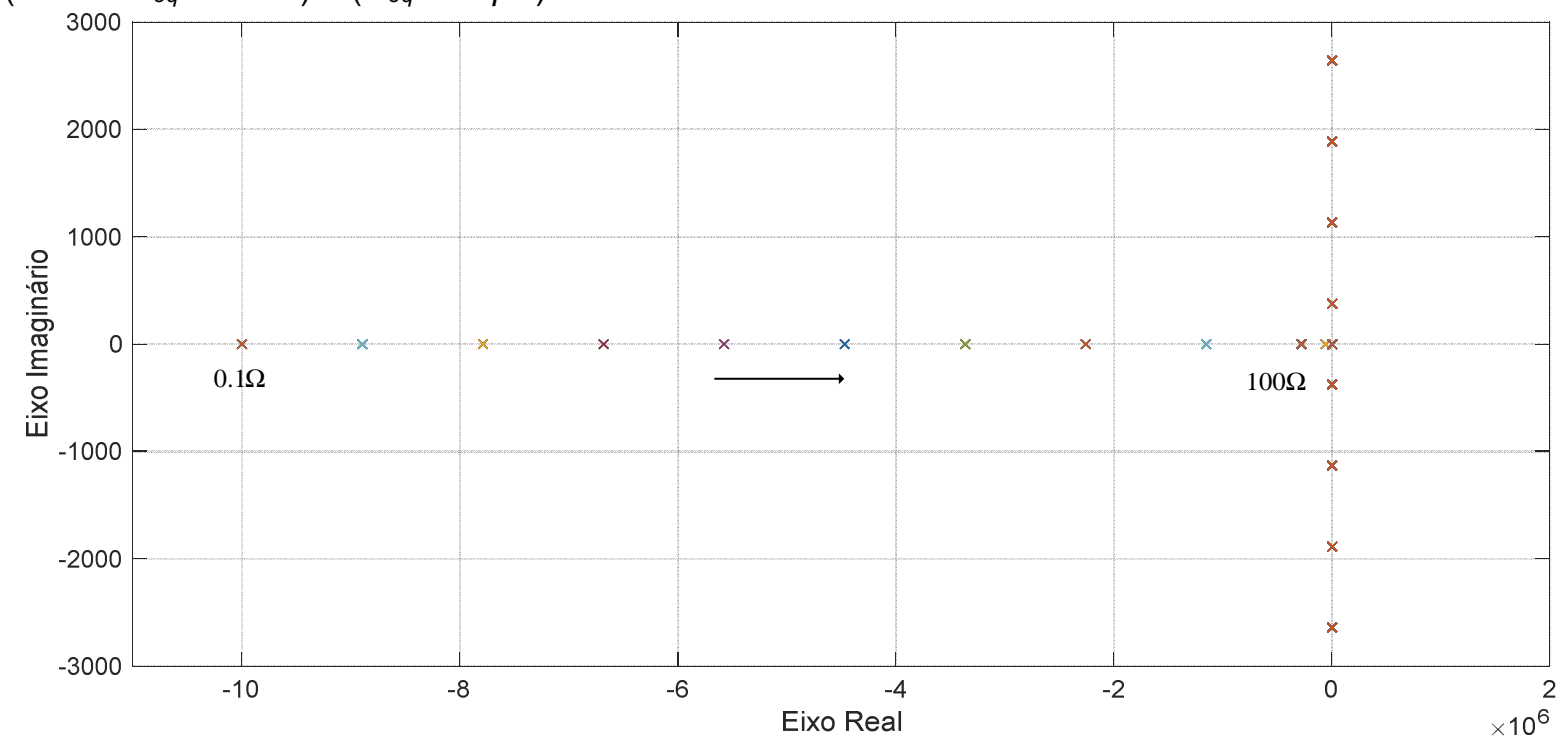

Fonte: Dados da pesquisa do autor.

Para o caso de variações na tensão da rede elétrica considerando o conversor série de potência, tem-se a diferença entre as tensões de entrada $v_{s}$ e de saída $v_{L}$ atribuído como distúrbio para a malha de controle de corrente, conforme apresentado na Figura 5.6, com a função de transferência em (5.22). Desse modo, é realizada uma mudança a partir do diagrama de blocos da Figura 5.6, que considera a corrente de saída do conversor série $i_{c s}$ como entrada e a tensão de entrada $v_{S}$ (rede elétrica) como saída do sistema.

A indutância é $L_{r d}$ e a resistência da rede é $R_{r d}$ também são consideradas. A função de transferência em malha fechada que representa a Figura 5.36 é dada por (5.74) (Campanhol, 2017).

Figura 5.36 - Diagrama de blocos das malhas de controle do conversor série para análise de estabilidade sob variações na tensão da rede elétrica

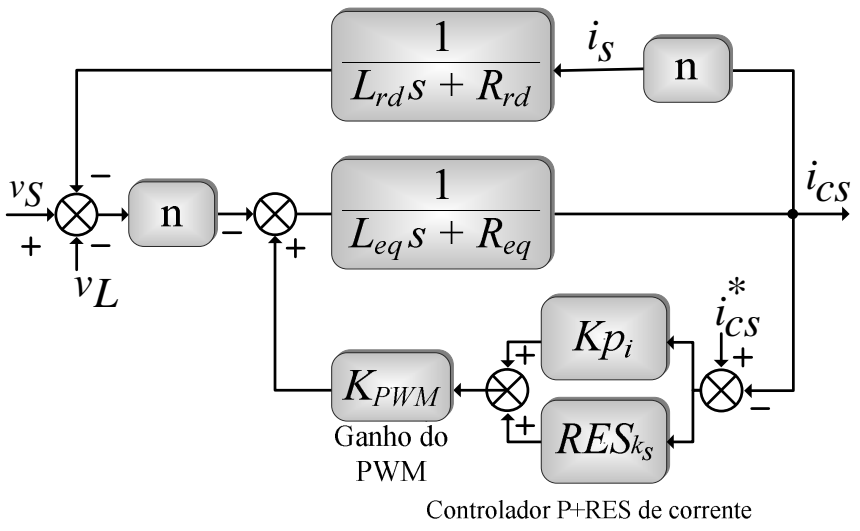

Fonte: Dados da pesquisa do autor. 


$$
G_{M F_{s i v}}=\frac{i_{c s}}{v_{S}}=\frac{A_{1} s+A_{2}}{B_{1} s^{2}+B_{2} s+B_{3}}
$$

em que:

$$
\begin{gathered}
A_{1}=L_{r d} n ; A_{2}=R_{r d} n ; B_{1}=L_{e q} L_{r d}+L_{r d} R E S_{k} i K p w m, \\
B_{2}=L_{e q} R_{r d}+L_{r d} R_{e q}+L_{r d} K p_{i} K p w m+R_{r d} R E S_{k} i K p w m ; B_{3}=R_{e q} R_{r d}+R_{r d} K p_{i} K p w m-n^{2} .
\end{gathered}
$$

A partir do critério de Routh-Hurwitz, a equação (5.74) tem todos os coeficientes do polinômio denominador positivo. Portanto, para ter essas condições, os parâmetros dos controladores $\mathrm{P}+\mathrm{RES}$, ou seja, $K p_{i}, R E S_{k} i$, devem ser ajustados. Assim, com os ganhos adquiridos em projeto, pode-se confirmar que a planta será estável mesmo com variações na tensão da rede elétrica.

$\mathrm{Na}$ Figura 5.37 e 5.38, são apresentados os polos da função de transferência em relação à equação (5.74) para valores diferentes de $L_{e q}$ e $R_{e q}$. Na Figura 5.37, considera-se $R_{e q}=0.5 \Omega$, e varia-se $L_{e q}$. Já na Figura 5.38, considera-se $L_{e q}=10 \mu \mathrm{H}$ , e varia-se $R_{e q}$. Assim, observa-se que todos os polos estão localizados no semiplano esquerdo do plano s. A estabilidade da planta não é alterada sob características diferentes da impedância da rede elétrica.

Figura 5.37 - Mapa de polos da FT em malha fechada sob análise de estabilidade do conversor série $\left(1 \mu H \leq L_{e q} \leq 2000 \mu H\right)$ e $\left(R_{e q}=0.5 \Omega\right)$

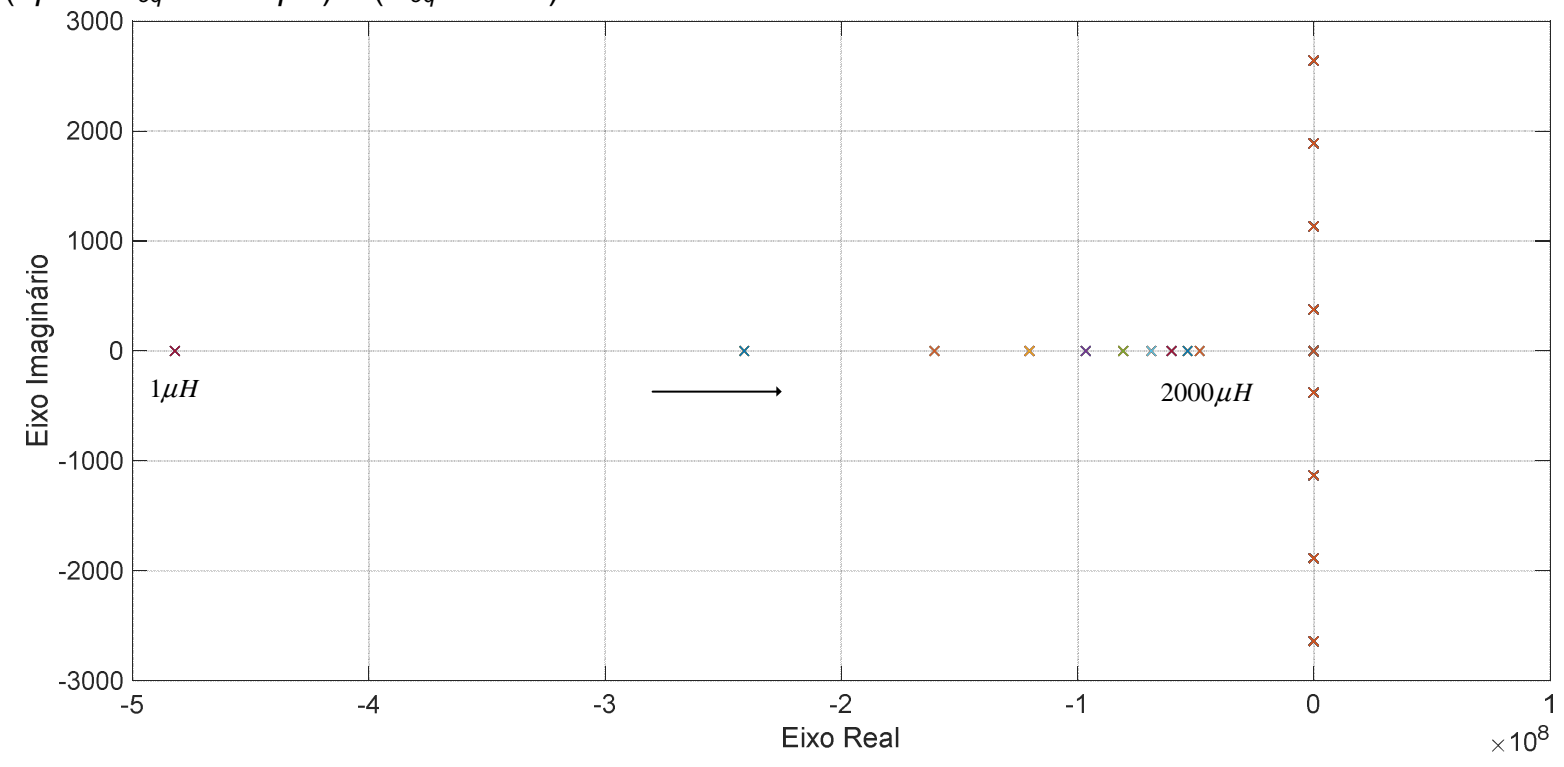

Fonte: Dados da pesquisa do autor. 
Figura 5.38 - Mapa de polos da FT em malha fechada sob análise de estabilidade do conversor série $\left(0.1 \Omega \leq \mathrm{R}_{e q} \leq 100 \Omega\right)$ e $\left(\mathrm{R}_{e q}=10 \mu H\right)$

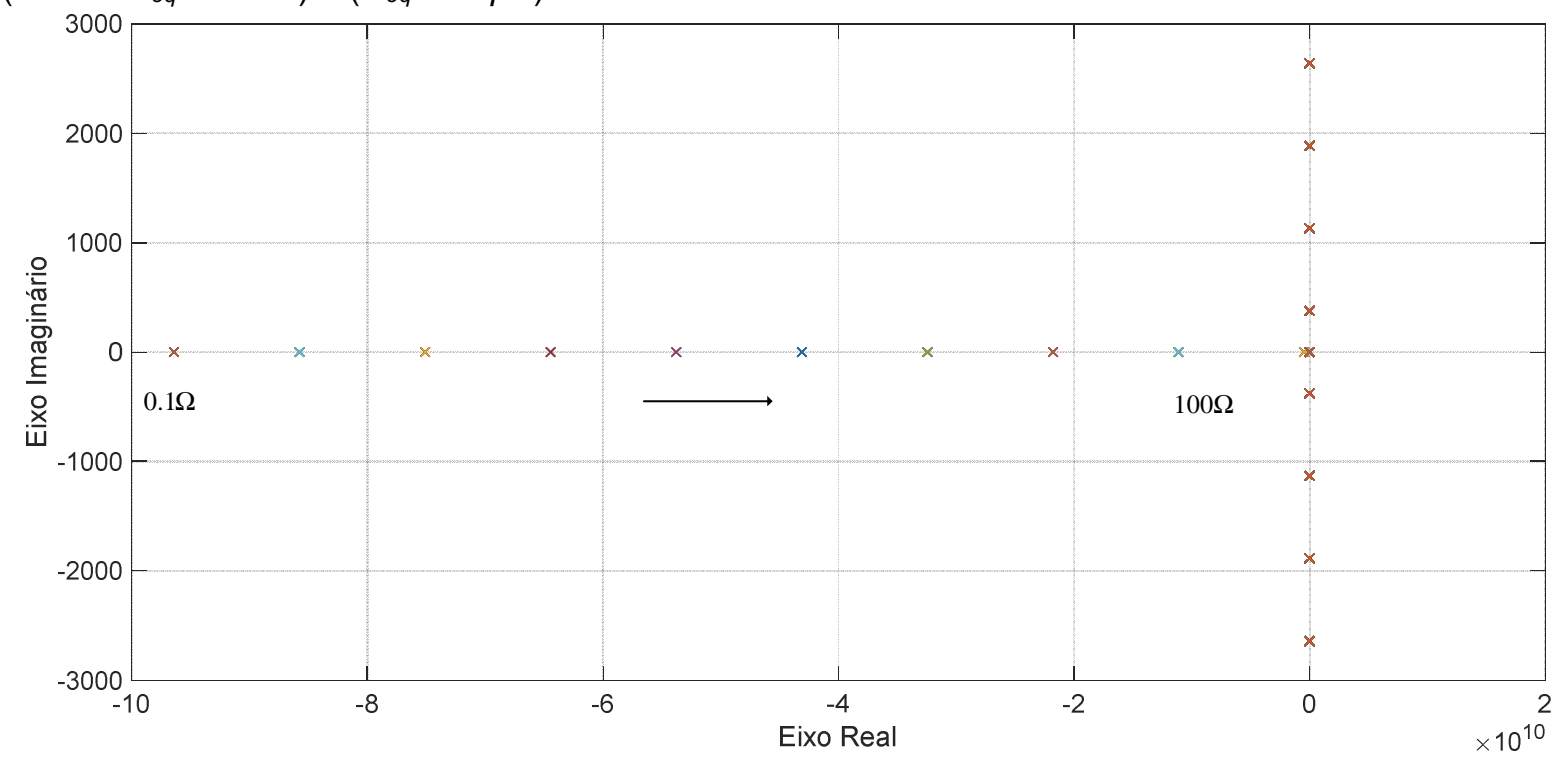

Fonte: Dados da pesquisa do autor.

A análise de estabilidade do conversor paralelo de potência, considerando-se as variações na tensão da rede elétrica, é realizada por meio da função de transferência em malha fechada apresentada em (5.36), com todos os coeficientes do polinômio denominador positivos, referente à Figura 5.10, a partir do critério de RouthHurwitz.

Portanto, para ter essas condições, os parâmetros dos controladores $\mathrm{P}+\mathrm{RES}$ e $\mathrm{P}$, ou seja, $K p_{v}, R E S_{k}, K p_{i}$, devem ser ajustados. Assim, com os ganhos adquiridos em projeto, pode-se confirmar que a planta será estável, mesmo com variações na tensão da rede. As variações na tensão da rede elétrica não interferem na estabilidade da mesma, devido o conversor sempre fornecer tensão senoidal à carga, ou seja, utiliza-se a estratégia de compensação dual.

\subsection{Considerações finais do capítulo}

Neste capítulo, foram apresentadas as modelagens matemáticas das plantas dos conversores estáticos de potência multiníveis série e paralelo, do elo CC e do desequilíbrio do elo CC. Além disso, apresentaram-se os modelos dos controladores que são utilizados nos conversores de potências série e paralelo e do elo CC. 
Os projetos dos controladores a partir da margem de fase e da frequência de cruzamento foram realizados, sendo um controlador proporcional ressonante para malha de controle do conversor série operando como fonte de corrente, um controlador proporcional para a malha interna de corrente e um controlador proporcional ressonante para a malha externa de tensão do conversor paralelo operando como fonte de tensão. Já para a malha total e desequilíbrio do elo CC, foram utilizados, na malha de controle, um controlador proporcional integral para cada.

Os estudos sobre o fluxo de potência do UPQC, que ocorrem por meio dos conversores série e paralelo, foram apresentados. As análises foram feitas para diferentes características, tais como potência reativa, distorção harmônica na corrente da carga, variação da tensão da rede e fator de carga do elo CC, todas mostradas por meio das curvas normalizadas das potências aparentes dos conversores.

Foi realizada a análise de estabilidade das malhas de controle do conversor série de corrente e do conversor paralelo de tensão, para distúrbios envolvendo variações da corrente da carga, bem como variações da tensão da rede elétrica. A partir desta análise, ficou comprovado que o sistema é estável para os respectivos ganhos dos controladores de ambos os conversores de potência. Constatou-se também que, mesmo com os distúrbios apresentados, o sistema opera em regime de estabilidade. 


\section{Simulações Computacionais}

Neste capítulo serão apresentados os resultados obtidos por meio de simulações computacionais, a partir das configurações do UPQC monofásico multinível tipo ANPC cinco níveis exibidas nos capítulos anteriores. Como plataforma de simulação computacional, escolheu-se o software PSIM.

\subsection{Introdução}

A fim de obter os resultados de simulação, foram implementados o circuito de potência utilizado nos dois conversores multiníveis ANPC cinco níveis para os conversores de potência série e paralelo, o algoritmo de sincronismo, os controladores de tensão e corrente para o conversor paralelo, o controlador de corrente para o conversor série, os algoritmos de geração de referência de tensão, bem como o algoritmo de geração de referência de corrente, baseado na Teoria da Potência Conservativa (CPT). Na figura 6.1, é mostrado o diagrama de blocos que representa o UPQC simulado.

Figura 6.1 - Diagrama de blocos do protótipo UPQC simulado.

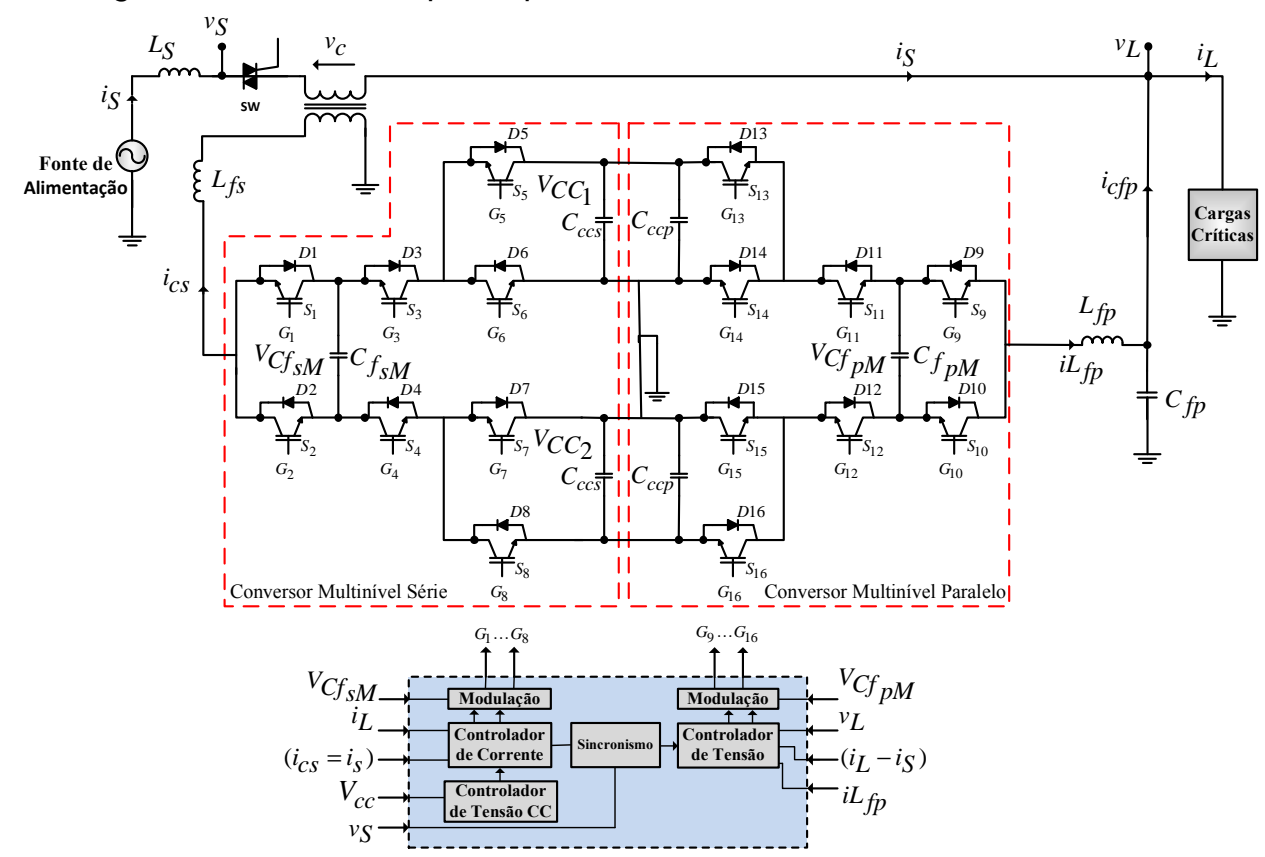

Fonte: Dados da pesquisa do autor. 
Para as simulações do UPQC, considerou-se uma carga não linear, constituída por um retificador em ponte completa a diodos alimentando uma carga $R L$ e $R C$.

Os comportamentos estático e dinâmico do UPQC são verificados a partir da presença de distúrbios da rede elétrica, tais como: harmônicos, elevações (swells) e afundamentos (sags) de tensão, assim como degraus de carga.

Na tabela 6.1 estão os parâmetros utilizados nas simulações.

Tabela 6.1 - Parâmetros de simulação

\begin{tabular}{|c|c|}
\hline Tensão eficaz nominal da rede elétrica & $63 \mathrm{~V}$ \\
\hline Valores eficazes da componente de $5^{\circ}$ harmônicos da tensão da rede elétrica & $6 \mathrm{~V}$ \\
\hline Valores eficazes da componente de $7^{\circ}$ harmônicos da tensão da rede elétrica & $5 \mathrm{~V}$ \\
\hline Tensão eficaz nominal de saída & $63 \mathrm{~V}$ \\
\hline Frequência da rede elétrica & $60 H z$ \\
\hline Tensão do barramento CC total & $250 \mathrm{~V}$ \\
\hline Indutância da rede elétrica & $10 m H$ \\
\hline Resistência da rede elétrica & $0,1 \Omega$ \\
\hline Indutância de acoplamento do conversor série & $4,33 m H$ \\
\hline Resistência de acoplamento do conversor série & $0,4 \Omega$ \\
\hline Relação de transformação do transformador de acoplamento série & $n=1$ \\
\hline Indutância de acoplamento do conversor paralelo & $1,3 m H$ \\
\hline Resistência do indutor de acoplamento do conversor paralelo & $0,21 \Omega$ \\
\hline Capacitância de filtragem do conversor paralelo & $80 \mu F$ \\
\hline Capacitância do barramento CC total & $9400 \mu F$ \\
\hline Frequência de chaveamento dos conversores de potência série e paralelo & $20 \mathrm{kHz}$ \\
\hline Resistência da carga & $17 \Omega$ \\
\hline Indutância da carga & $280 m H$ \\
\hline Capacitor da carga & $940 \mu F$ \\
\hline
\end{tabular}

Fonte: Dados da pesquisa do autor. 


\subsection{Resultados de Simulações}

As figuras 6.2 (a) e (b) mostram as tensões e corrente do inversor 5L-ANPC de tensão, em malha aberta, com uma carga resistiva, ou seja, a tensão do capacitor de fase $V_{C f, M}$, tensão no inversor $V_{i n v}$, tensão de saída $V_{o}$ e corrente de fase do capacitor $I_{C f}$. Nota-se que a tensão sobre o capacitor de fase $V_{C f, M}$ está controlada e não sofre variações, além de a tensão do inversor $V_{i n v}$ apresentar os cinco níveis, em conformidade com a metodologia abordada no capítulo 3.

Figura 6.2 - Inversor de tensão 5L-ANPC em malha aberta: (a) tensão do capacitor de fase $V_{C f, M}$, tensão no inversor $V_{i n v}$, tensão de saída $V_{o}$ e corrente no capacitor de fase $I_{C f}$; (b) tensão no inversor $V_{i n v}$, tensão de saída $V_{o}$ e corrente no capacitor de fase $I_{C f}$.

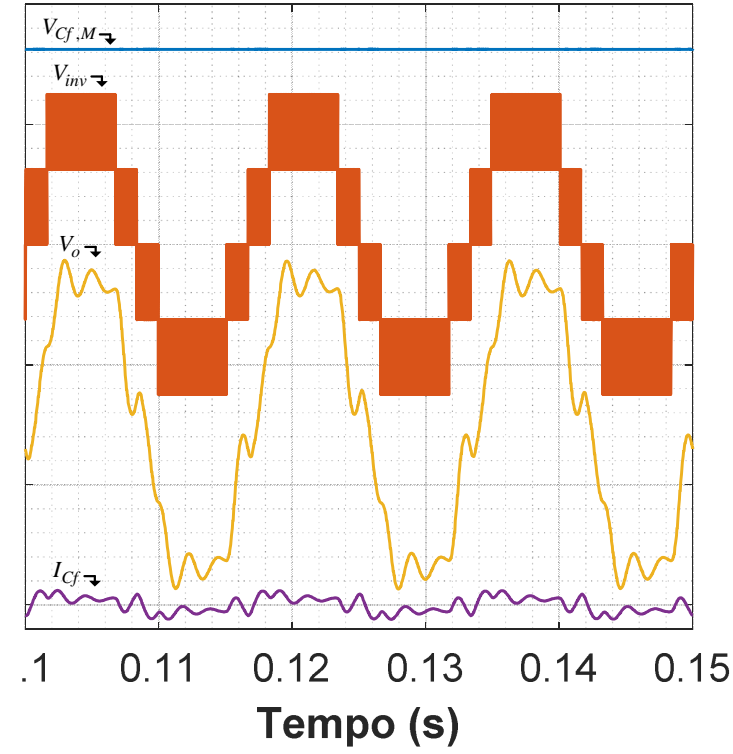

(a)

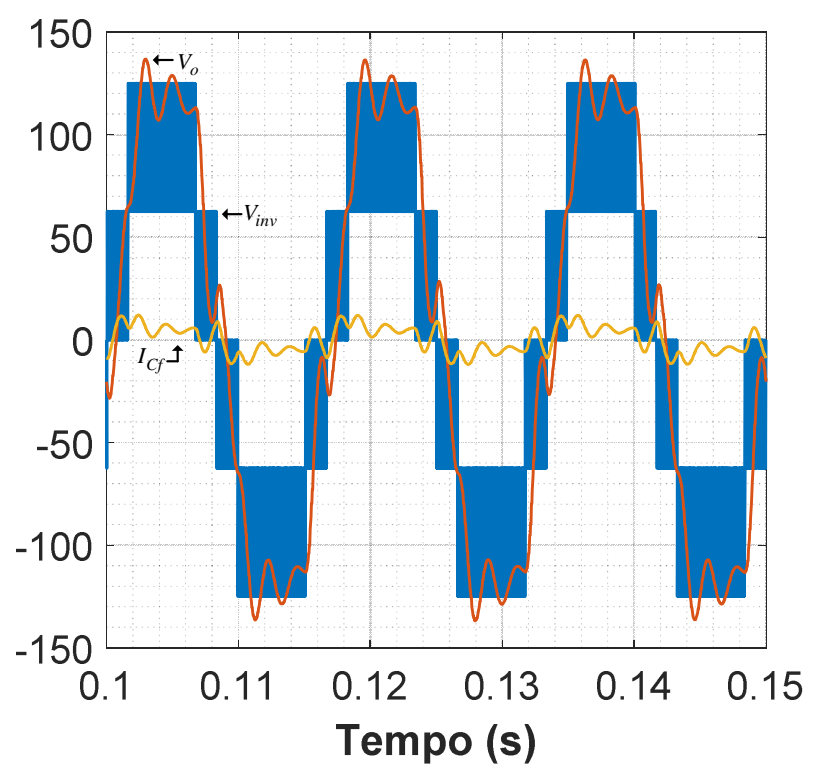

(b)

Fonte: Dados da pesquisa do autor.

As figuras 6.3 (a) e (b) mostram as tensões e as correntes do UPQC com carga RL e RC, ou seja, a tensão de entrada $v_{S}$ com DHT de 1,45\%, a tensão de saída do conversor paralelo $v_{L}$ com DHT de 1,7\% para RL e 1,9\% para RC e a tensão sobre o transformador de acoplamento série $v_{C}$, a corrente de entrada já compensada $i_{S}$ com DHT de 1,83\% para RL e 1,97\% para RC, a corrente compensada fornecida pelo conversor paralelo $i_{C f p}$ e a corrente na carga não compensada $i_{L}$ com DHT de $46 \%$ 
para $\mathrm{RL}$ e $69 \%$ para $\mathrm{RC}$. Constata-se que a tensão e a corrente de entrada é senoidal e com baixo conteúdo harmônico.

Figura 6.3 - UPQC: (a) e (b) tensão de entrada $v_{S}$, tensão de saída $v_{L}$ e tensão sobre o transformador de acoplamento $v_{C}$, corrente de entrada $i_{s}$, corrente de compensação paralela $i_{c f p}$ e corrente de carga $i_{L}$; (a) carga - RL, (b) carga - RC.
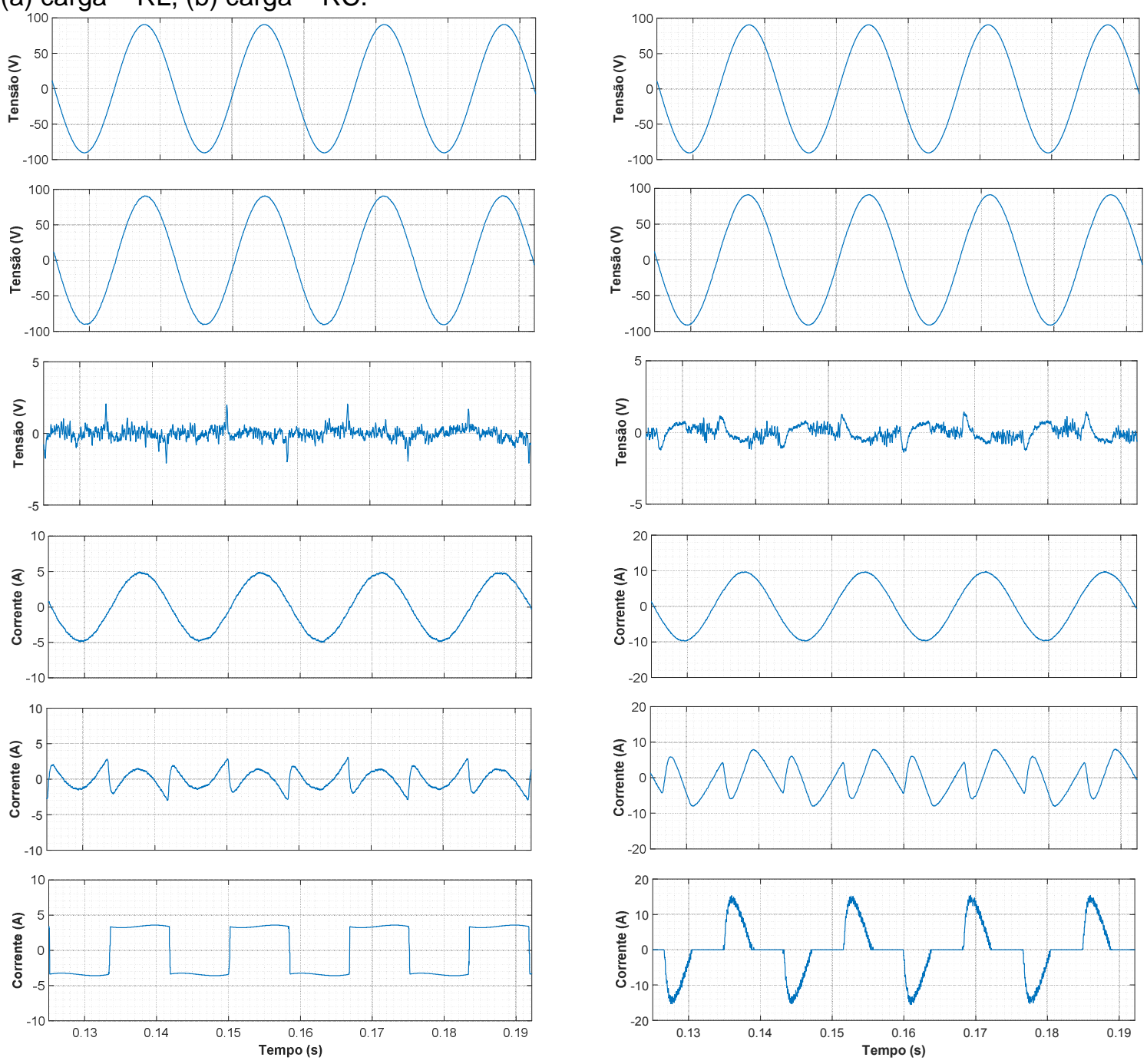

(a)

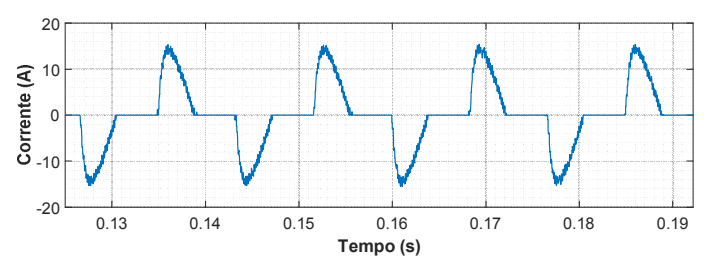

(b)

Fonte: Dados da pesquisa do autor.

Na Figura 6.4 (a) e (b), mostram-se a tensão de entrada $v_{S}$ em fase com a tensão de saída $v_{L}$ e a tensão sobre o transformador de acoplamento série $v_{C}$, que representa a diferença entre $v_{S}$ e $v_{L}$, que já era previsto para esse tipo de estrutura. Na Figura 6.4 (a), utiliza-se carga RL e, na Figura 6.4 (b), carga RC. 
Figura 6.4 - UPQC: tensão de entrada $v_{S}$, tensão de saída $v_{L}$ e tensão sobre o transformador de acoplamento; $v_{C}$; (a) carga RL; (b) carga RC.

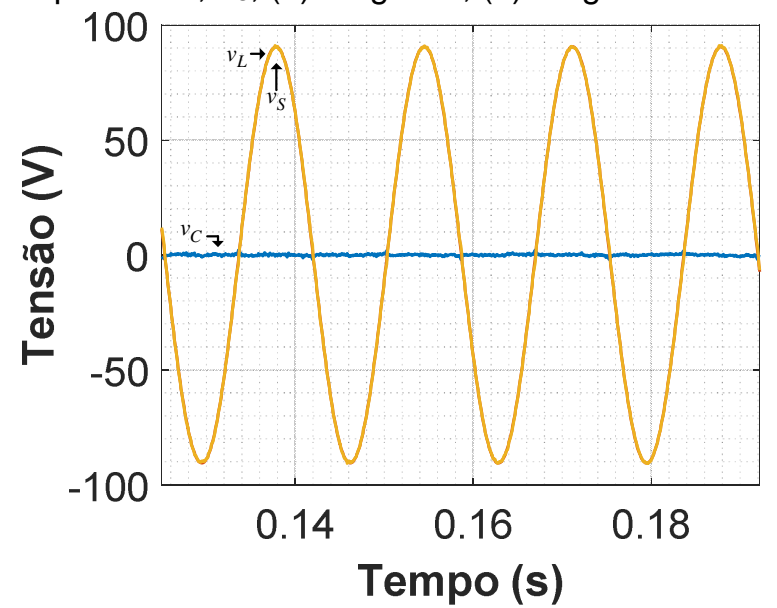

(a)

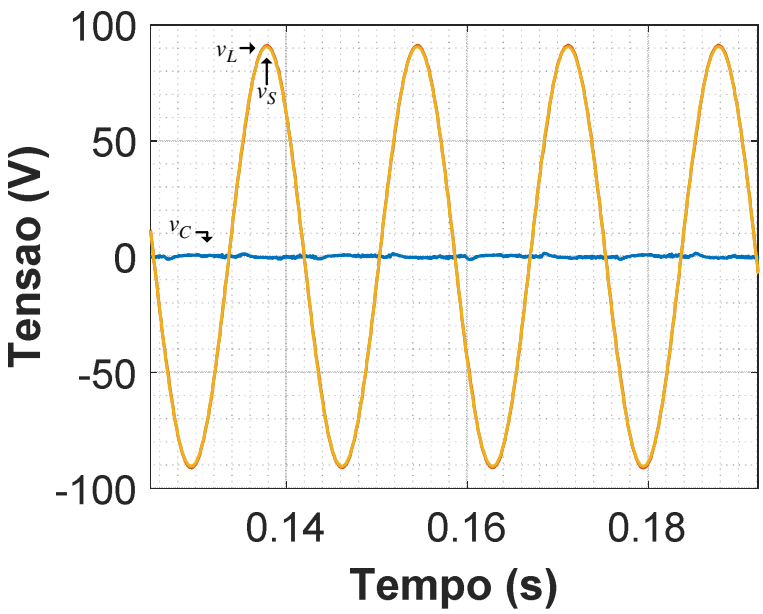

(b)

Fonte: Dados da pesquisa do autor.

As figuras 6.5 (a) e (b) mostram as tensões e correntes do UPQC com carga $\mathrm{RL}$ e RC, ou seja, a tensão de entrada $v_{S}$ distorcida com $8,72 \%$, tensão de saída do conversor paralelo $v_{L}$ com DHT de $1,77 \%$ com carga $\mathrm{RL}$ e 1,95\% com carga RC, a corrente de entrada já compensada $i_{S}$ com DHT de 3,39\% com carga RL e 2,17\% com carga RC e a corrente na carga $i_{L}$ com DHT de $46 \% \%$ com carga RL e $69 \%$ com carga RC. As correntes de entrada, compensadas tanto com cargas $R L$ e $R C$, possuem baixo conteúdo harmônico, ao passo que as tensões estão em fase com as correntes.

Figura 6.5 - UPQC (a) e (b) tensão de entrada $v_{S}$, tensão de saída $v_{L}$ e tensão sobre o transformador de acoplamento $v_{C}$, corrente de entrada $i_{S}$, corrente de compensação paralela $i_{c f p}$ e corrente de carga $i_{L} ;$ (a) carga - RL, (b) carga - RC.
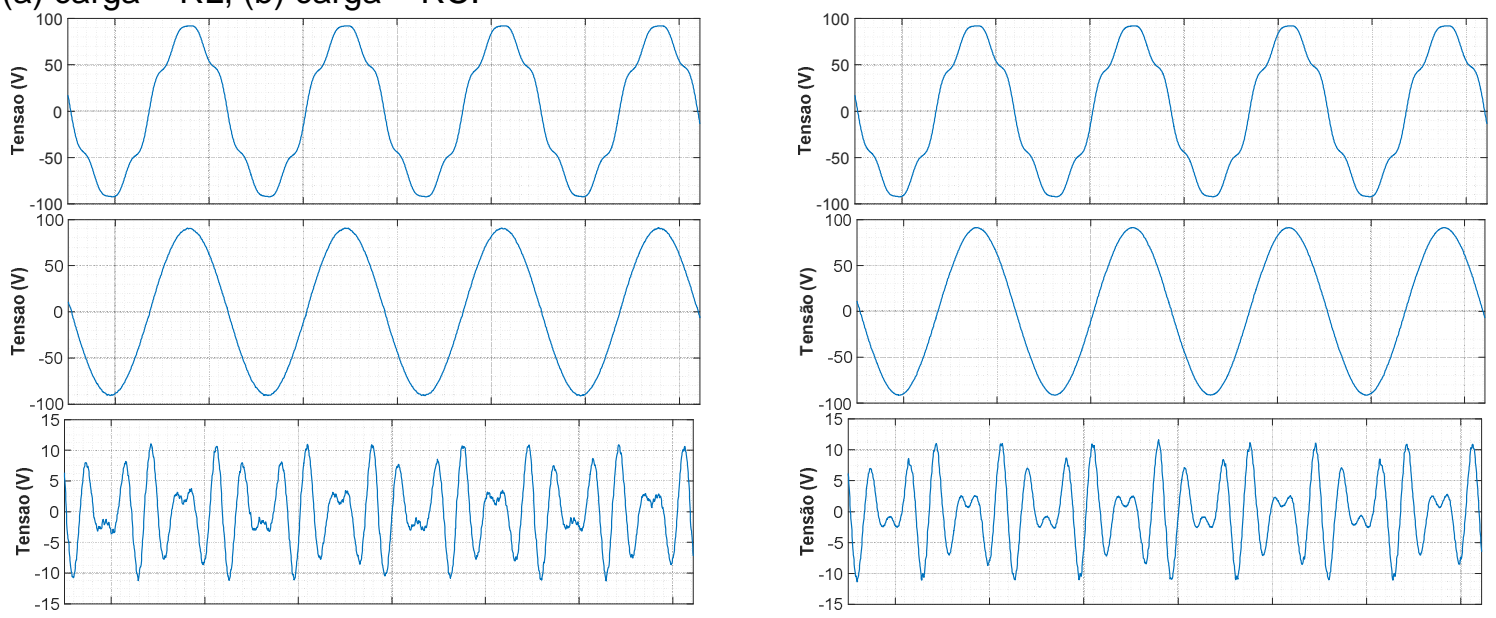


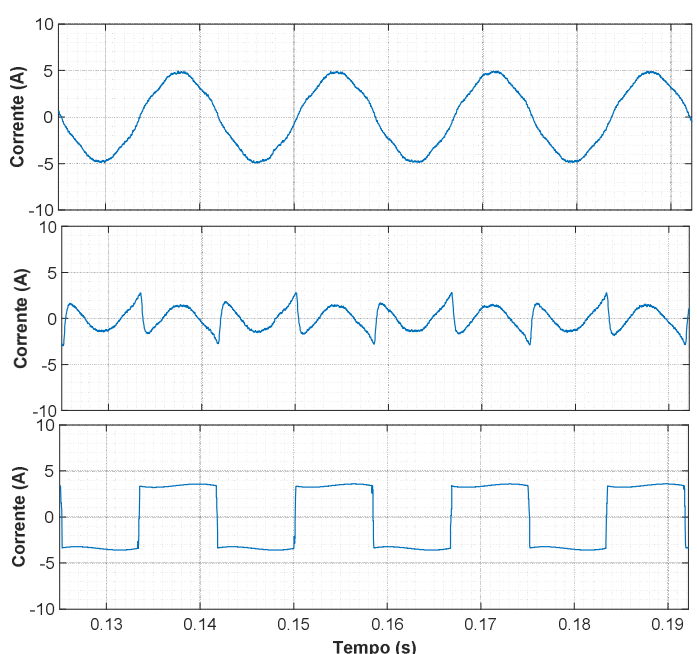

(a)

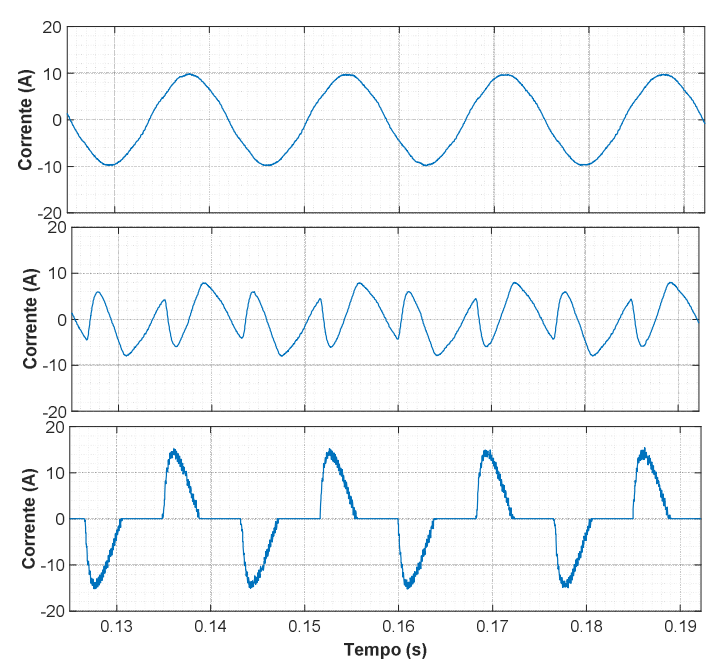

(b)

Fonte: Dados da pesquisa do autor.

Na Figura 6.6, mostram-se a tensão de entrada distorcida $v_{S}$ com 8,72\% em fase com a tensão de saída $v_{L}$ e a tensão sobre o transformador de acoplamento série $v_{C}$, que representa a diferença entre $v_{S}$ e $v_{L}$. Na Figura 6.6 (a), utiliza-se carga $\mathrm{RL}$ e na Figura 6.6 (b) carga $R C$.

Figura 6.6 - UPQC com conteúdo harmônico em $v_{S}$ : (a) tensão de entrada $v_{S}$, tensão de saída $v_{L}$ e tensão sobre o transformador de acoplamento, $v_{C}$.

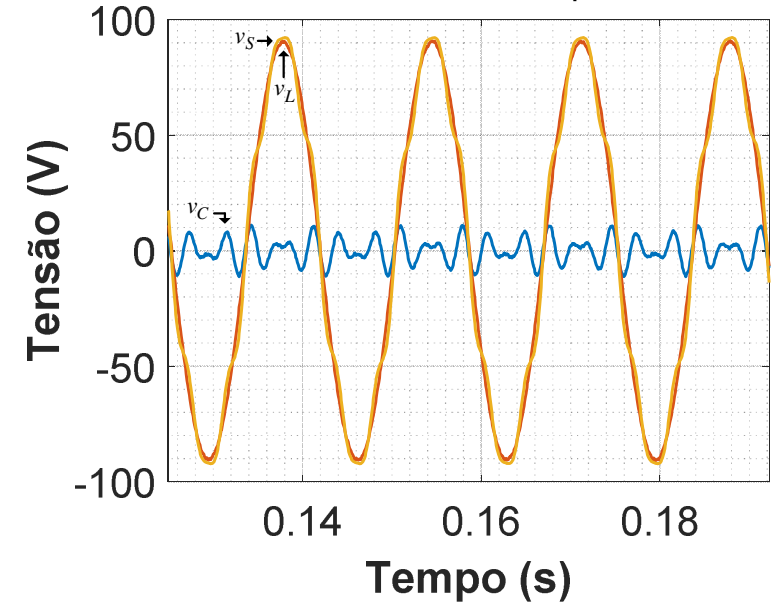

(a)

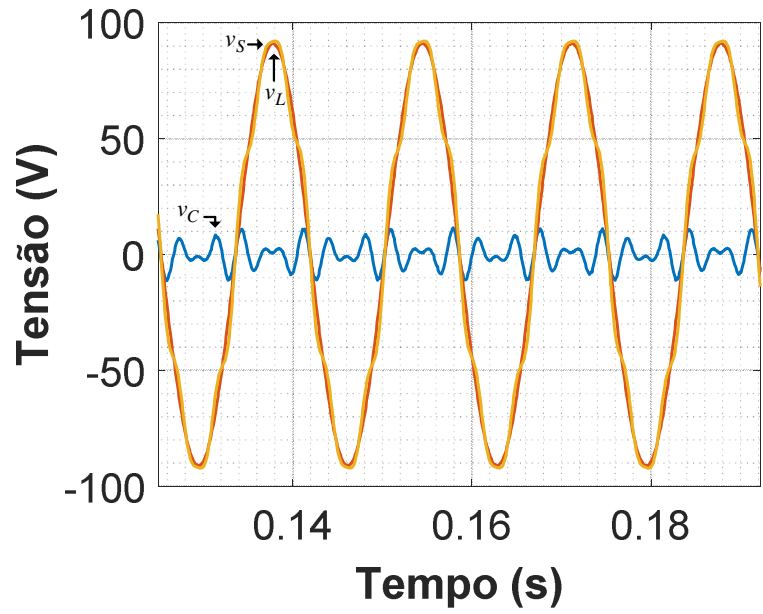

(b)

Fonte: Dados da pesquisa do autor.

Nas figuras 6.7 ,6.8, 6.9 e 6.10, verifica-se a regulação da tensão de saída do UPQC, mediante variações da tensão de entrada $v_{S}$. Nas figuras 6.7 e 6.8 , observase que não há distorção na tensão de entrada. Já nas figuras 6.9 e 6.10, a DHT é de $8,42 \%$. Entre 0,2 a $0,29 \mathrm{~s}$, têm-se um afundamento na tensão de entrada $\left(v_{S}=54,08 \mathrm{~V}\right)$, 
ou seja, $v_{S}<v_{L}$. Entre 0,385 a 0,485s, têm-se uma elevação na tensão de entrada ( $v_{S}=72,45 \mathrm{~V}$, ou seja, $v_{S}>v_{L}$. Verifica-se que a tensão de saída $v_{L}$ sempre é regulada para um valor eficaz de 63,63V, independentemente do valor da tensão de entrada. Nas figuras 6.7 e 6.9, utiliza-se carga $R L$ e, nas figuras 6.8 e 6.10, utiliza-se carga RC.

Figura 6.7 - UPQC: (a) e (b) $v_{S}>v_{L}$ e $v_{S}<v_{L}$ : tensão de entrada $v_{S}$, tensão de saída $v_{L}$ e tensão sobre o transformador de acoplamento; $v_{C}$; corrente de entrada is e corrente de carga $i_{L}$; carga RL.

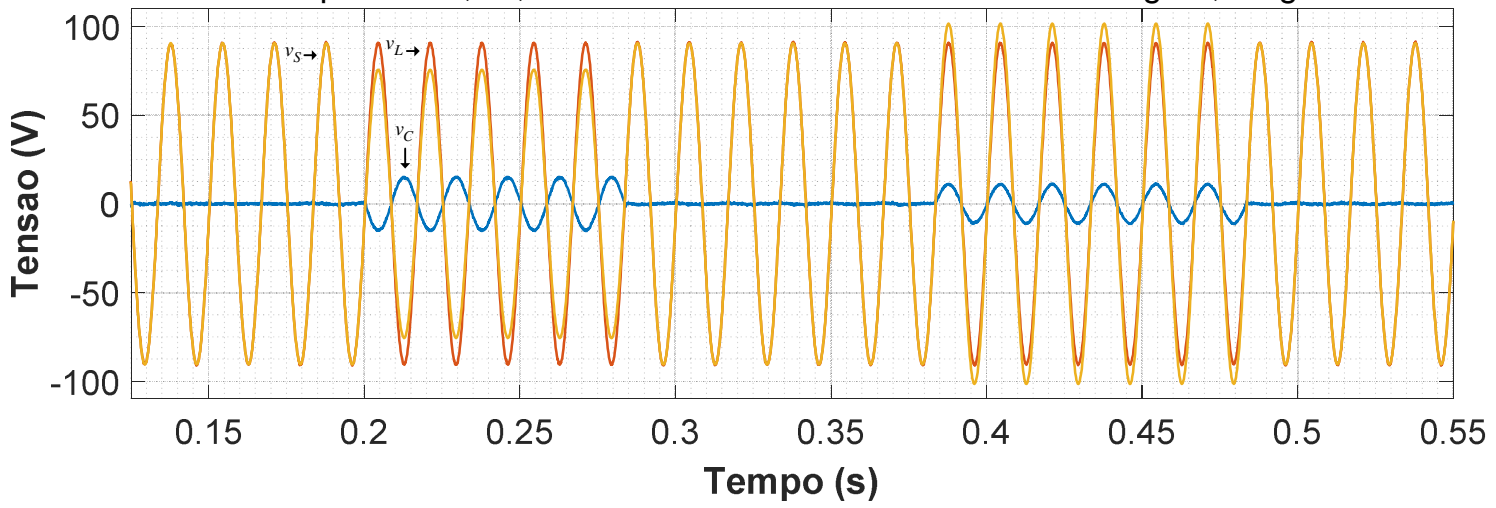

(a)

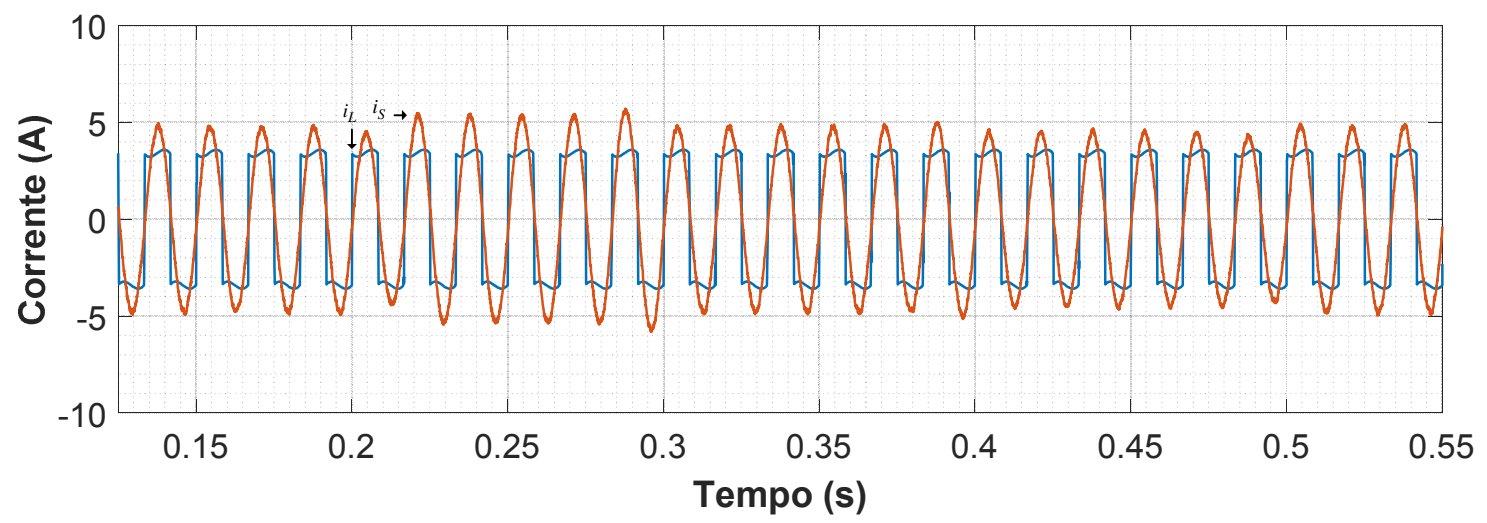

Fonte: Dados da pesquisa do autor.

(b)

Figura 6.8 - UPQC: (a) e (b) $v_{S}>v_{L}$ e $v_{S}<v_{L}$ : tensão de entrada $v_{S}$, tensão de saída $v_{L}$ e tensão sobre o transformador de acoplamento; $v_{C}$; corrente de entrada is e corrente de carga $i_{L}$; carga RC.

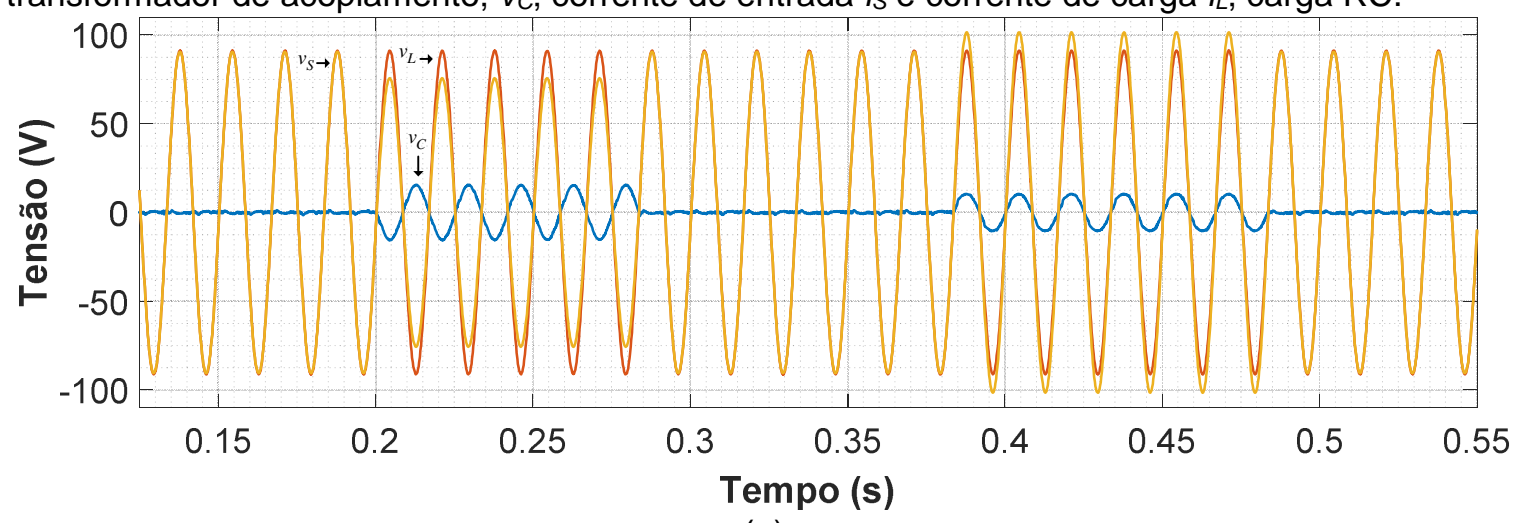

(a) 


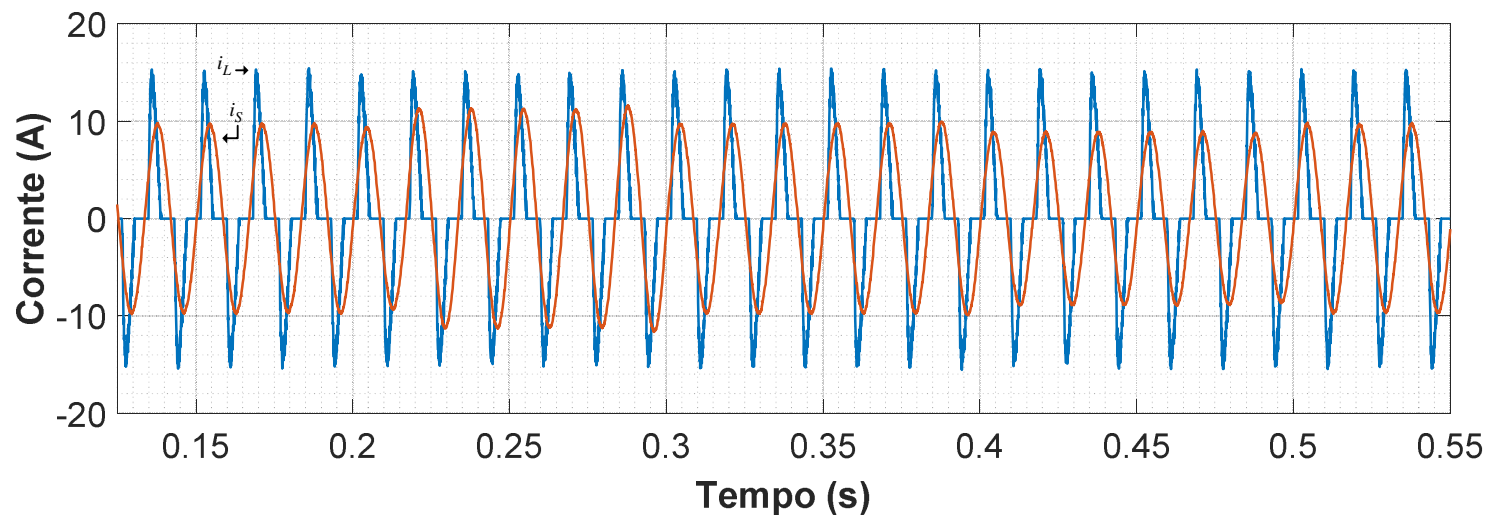

(b)

Fonte: Dados da pesquisa do autor.

Figura 6.9 - UPQC: (a) e (b) $v_{S}>v_{L}$ e $v_{S}<v_{L}$ : tensão de entrada $v_{S}$ com DHT de 8,42\%, tensão de saída $v_{L}$ e tensão sobre o transformador de acoplamento; $v_{C}$; corrente de entrada is e corrente de carga $i_{L} ;$ carga RL.

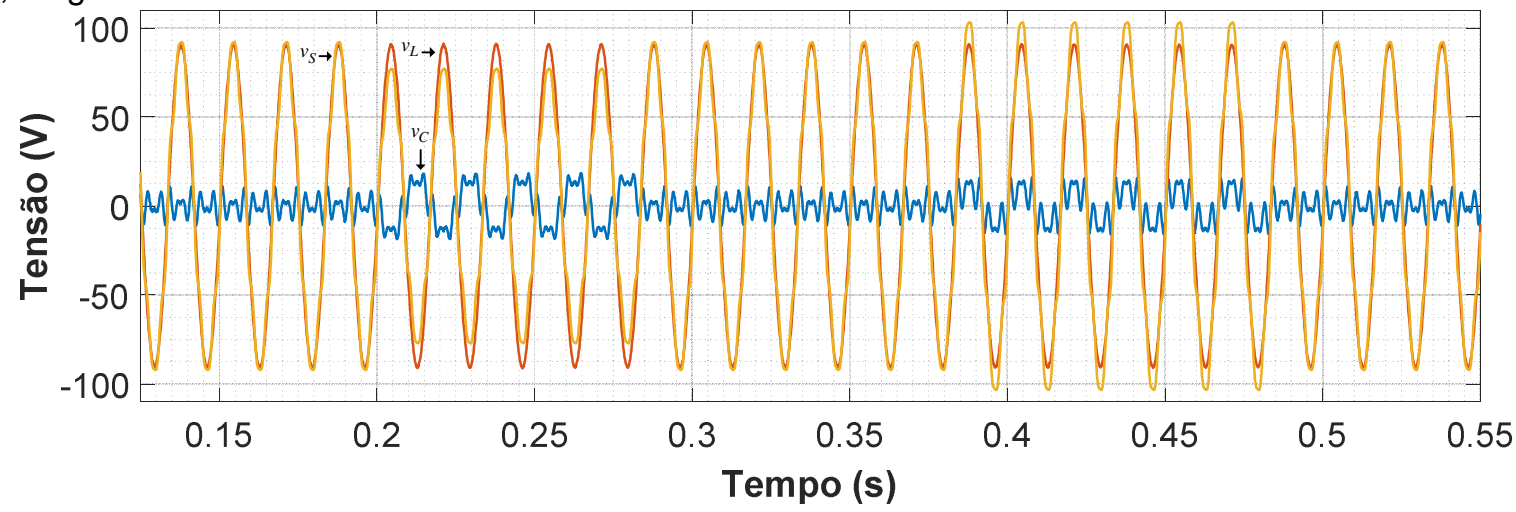

(a)

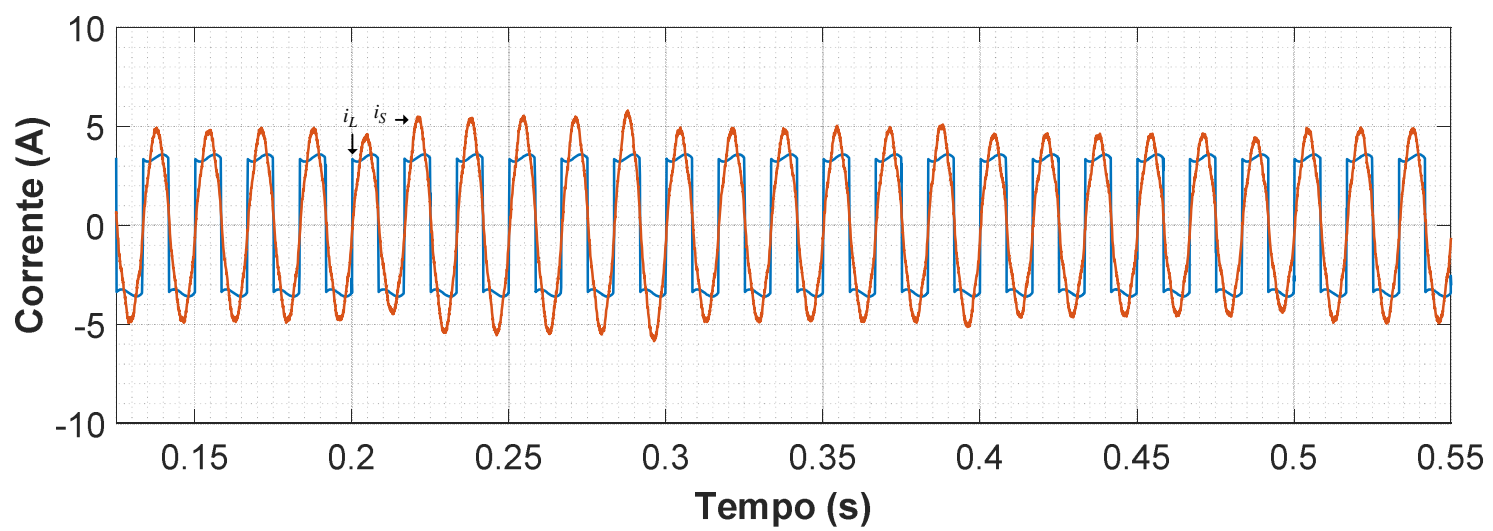

(b)

Fonte: Dados da pesquisa do autor. 
Figura 6.10 - UPQC: (a) e (b) $v_{S}>v_{L}$ e $v_{S}<v_{L}$ : tensão de entrada $v_{S}$ com DHT de $8,42 \%$, tensão de saída $v_{L}$ e tensão sobre o transformador de acoplamento; $v_{C}$; corrente de entrada is e corrente de carga $i_{L}$; carga RC.

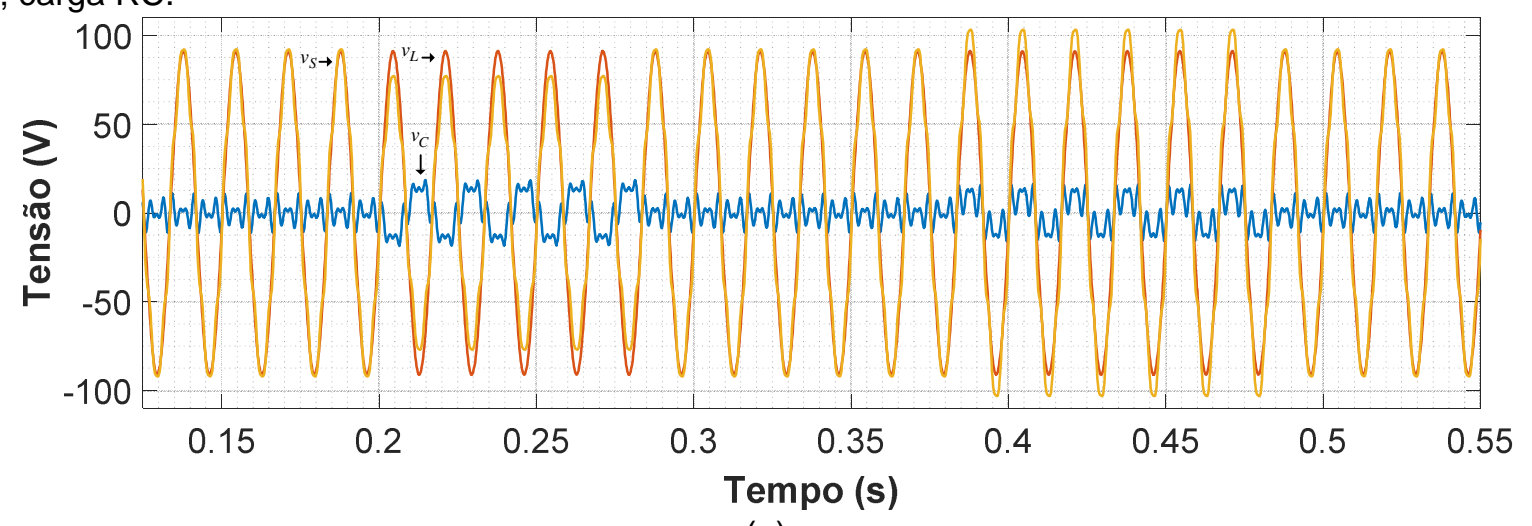

(a)

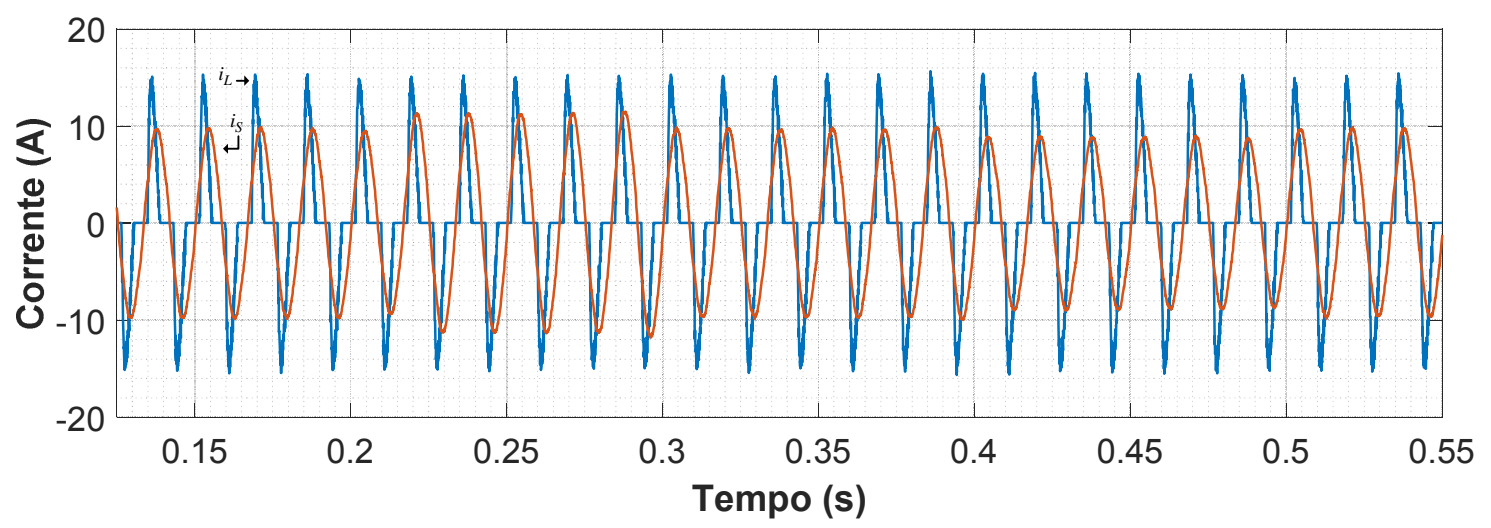

Fonte: Dados da pesquisa do autor.

(b)

Nota-se que a amplitude da corrente de entrada varia, quando se têm variações da tensão de entrada $v_{S}$ de acordo com o seu nível, ou seja, quando a tensão de entrada é maior ou menor que a tensão de saída, como mostram as figuras 6.7 a 6.10 (b), de forma a manter o fluxo de potência no UPQC.

A tensão que aparece sobre o transformador de acoplamento série é decorrente da diferença entre a tensão de entrada e da tensão de saída, ou seja, ela é dada pela diferença de tensão que aparece sobre o transformador série. Enquanto na tensão de entrada surgem harmônicos, afundamentos e elevações de tensão, observa-se que, na tensão de saída, a forma de onda é sempre senoidal, regulada e com baixa DHT.

Desse modo, mesmo não estabelecendo um controle específico de tensão sobre o transformador série, o mesmo já é controlado indiretamente pelo conversor paralelo de tensão. Quando não há diferença entre as tensões de entrada e de saída, a tensão sobre o transformador de acoplamento série tende a se tornar muito pequena. 
No período do afundamento da tensão de entrada, aparece uma potência ativa média que é drenada do barramento CC e fornecida para a rede elétrica por meio do conversor série. No período da elevação da tensão de entrada, surge uma potência ativa média que é drenada da rede elétrica por meio do conversor série, realizando, assim, o controle do fluxo de potência. Desse modo, a potência média será absorvida ou fornecida pelo conversor estático de potência série.

As figuras 6.11 (a) e (b) mostram o comportamento dinâmico de acordo com as variações de carga RL e RC no UPQC, ou seja, a tensão de entrada $v_{S}$, a tensão de saída $v_{L}$, a corrente de entrada já compensada $i_{S}$, a corrente compensada fornecida pelo conversor paralelo $i_{C f p}$ e a corrente na carga não compensada $i_{L}$. Na figura 6.11 (a), a carga utilizada é $R L$ e, na Figura 6.11 (b), a carga é RC. São realizadas variações de $50 \%$ a $100 \%$ em 0,15 s e, em seguida, de $100 \%$ a $50 \%$ em 0,25 s do seu valor nominal.

Figura 6.11 - UPQC com degrau de carga: (a) e (b) tensão de entrada $v_{S}$, tensão de saída $v_{L}$, corrente de entrada $i_{S}$, corrente de compensação paralela $i_{c f p}$ e corrente de carga $i_{L}$; (a) carga RL; (b) carga RC.

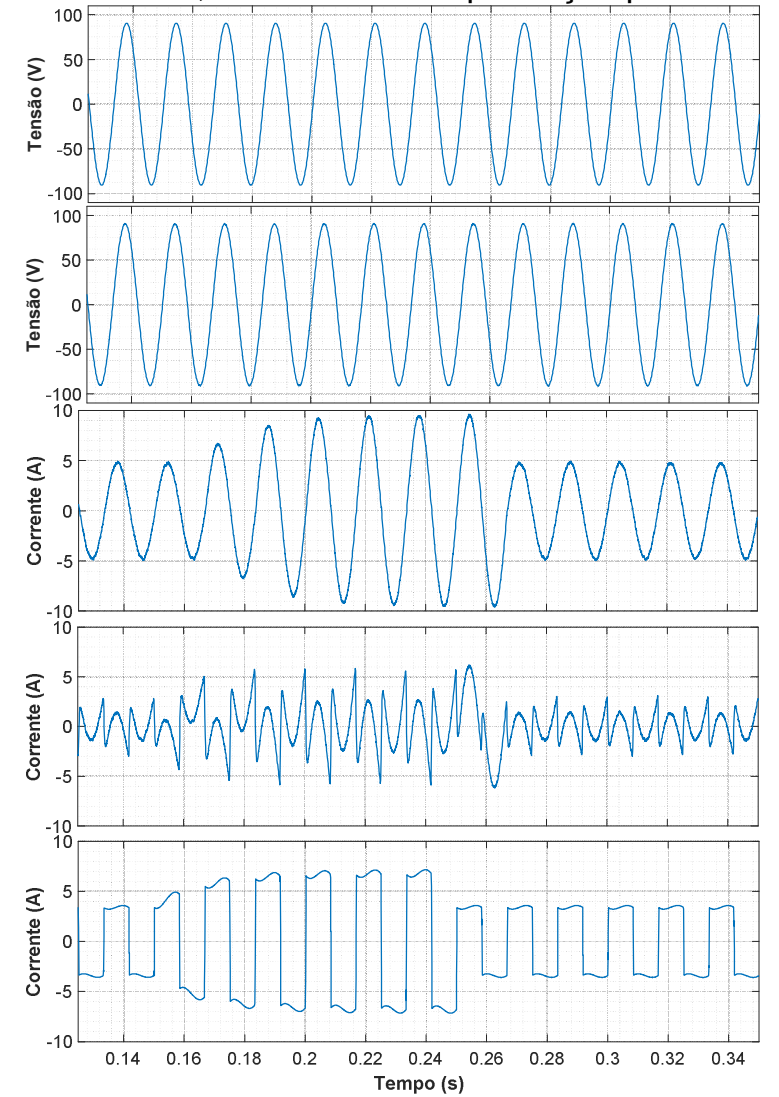

(a)

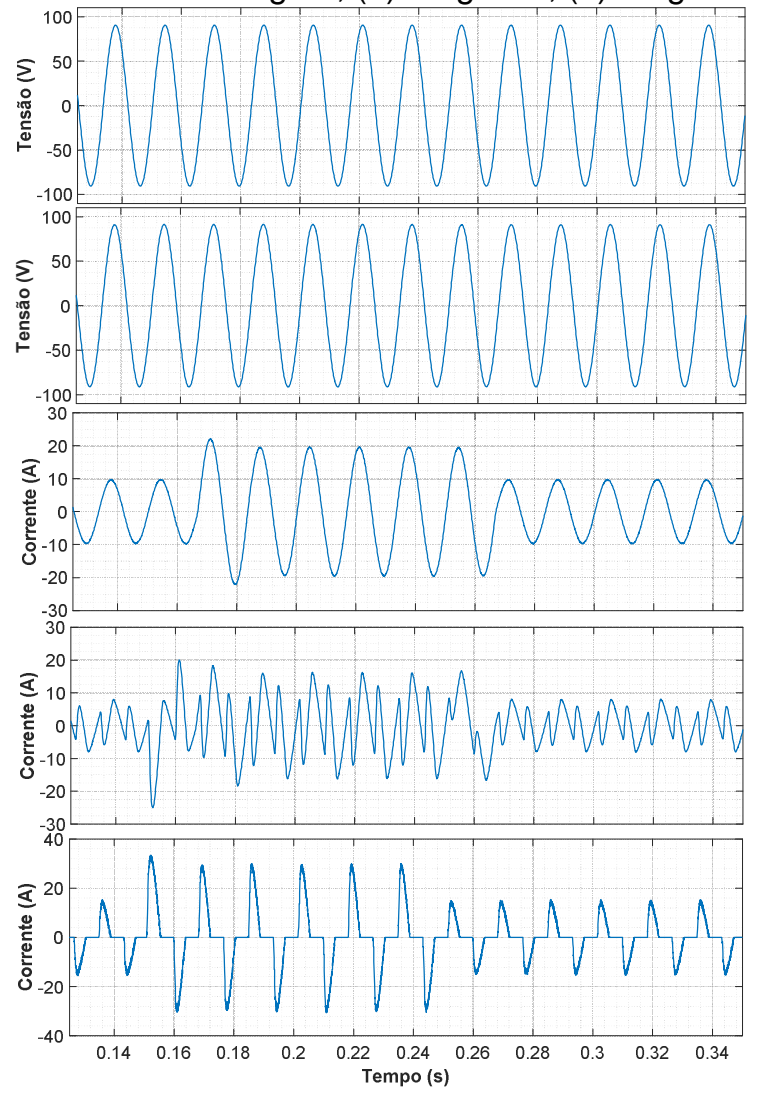

(b)

Fonte: Dados da pesquisa do autor. 
A Figura 6.12 (a) e (b) mostra o comportamento dinâmico de acordo com as variações de carga no UPQC, conforme a tensão do elo CC total $V_{C C_{T}}$, a tensão dos elos superior $V_{C C_{1}}$ e inferior $V_{C C_{2}}$ e a corrente de entrada $i_{S}$. Varia-se a carga de $50 \%$ a $100 \%$ do seu valor nominal em 0,5s. Nota-se uma redução nas tensões do elo CC e um aumento da amplitude da corrente de entrada. Em seguida, a carga é variada de $100 \%$ a $50 \%$ do seu valor nominal em 1,5s. Observa-se que há uma elevação nas tensões do elo CC e uma diminuição da corrente de entrada.

Figura 6.12 - UPQC com degrau de carga: (a) e (b) tensão do elo total CC $V_{C C T}$, tensão do elo superior $V_{C C 1}$ e tensão do elo inferior $V_{C C 2}$, corrente de entrada is; (a) carga RL; (b) carga RC.

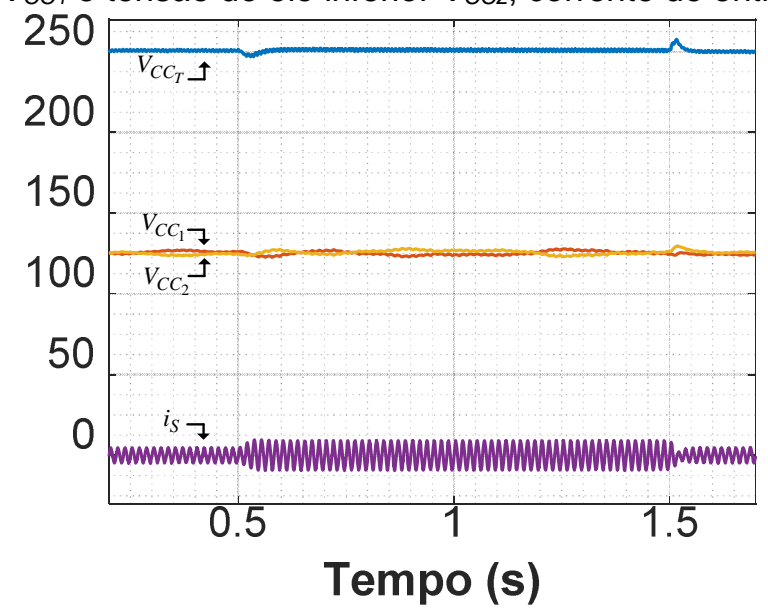

(a)

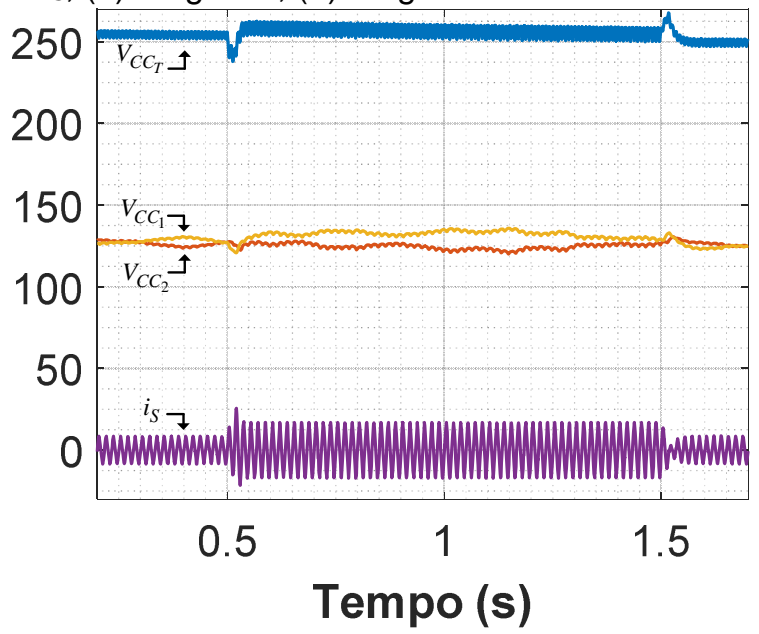

(b)

Fonte: Dados da pesquisa do autor.

\subsection{Considerações finais do capítulo}

Neste capítulo foram apresentados os resultados de simulação do UPQC monofásico, em que as simulações computacionais foram realizadas por meio da ferramenta computacional PSIM.

$\mathrm{Na}$ composição do UPQC, foram utilizados dois conversores multiníveis tipo ANPC de cinco níveis, um para o conversor série de potência e outro para o conversor paralelo de potência.

No conversor série, utilizaram-se um filtro indutivo e um transformador de acoplamento monofásico com relação de 1:1 conectado em série com o sistema. Já para o conversor paralelo, foi utilizado um filtro LC conectado à saída do conversor. 
Como carga, utilizou-se um retificador em ponte completa a diodos alimentando uma carga $\mathrm{RL}$ e $\mathrm{RC}$, que é uma carga não linear. Além disso, mostrou-se a pré-carga do elo CC, consistindo na partida do UPQC.

Os comportamentos estáticos e dinâmicos foram mostrados com a presença de distúrbios da rede elétrica, tais como harmônicos, afundamentos e elevações de tensões, bem como degraus de carga.

De acordo com os aspectos construtivos e definidos, os modos de operação do UPQC para ambos os conversores, sendo a estratégia de compensação dual, notase que a tensão da saída, ou seja, a tensão na carga, sempre será senoidal, regulada e com baixa DHT.

Dessa maneira, os harmônicos, afundamentos e elevações de tensão, bem como perturbações da rede elétrica, sempre serão absorvidos pelo transformador de acoplamento série, ou seja, o transformador do conversor série de potência irá absorver de forma natural essas perturbações devido estar entre a tensão de entrada e de saída, ou seja, são indiretamente compensados.

Sendo assim, não há necessidade de calcular qualquer referência de tensão de compensação para conversor série de potência.

No caso específico em que a tensão de entrada apresenta formato senoidal, com pequenas distorções harmônicas e amplitude muito próxima da tensão de saída, a tensão sobre o transformador série será muito pequena também, ou seja, próximo de zero.

A partir dos resultados apresentados, é possível verificar o funcionamento das malhas de controle das tensões e das correntes, por meio dos projetos e dos modelos dos controladores nos comportamentos estáticos e dinâmicos.

O sistema UPQC proposto realizou de forma efetiva as reduções das taxas de distorções harmônicas na corrente de entrada, e na tensão de saída. Dessa forma, observou-se a supressão dos harmônicos, de forma que a corrente compensada da rede elétrica é senoidal com baixa DHT e regulada, bem como a tensão imposta pelo conversor paralelo de potência. Vale ressaltar que tais resultados encontram-se em conformidade com as recomendações estabelecidas no PRODIST do IEEE 519. 


\section{Resultados Experimentais}

\subsection{Introdução}

Este capítulo apresenta os resultados experimentais obtidos de ensaios conduzidos em um protótipo UPQC monofásico em modo dual, implementado no Laboratório de Fontes Alternativas e Processamento de Energia LAFAPE/SEL/EESC/USP.

Com o intuito de validar os estudos teóricos mostrados nos capítulos anteriores, os resultados experimentais são extraídos com o uso de uma carga não linear, constituída por um retificador monofásico não controlado em ponte completa, alimentado por uma carga RL e RC.

Os comportamentos estático e dinâmico são analisados de acordo com a presença de distúrbios da rede elétrica, tais como harmônicos, elevações e afundamentos de tensão, e degraus de carga. O condicionamento de corrente é verificado levando-se em consideração a eliminação de harmônicos e compensação de reativos da carga. Além disso, o fluxo de potência nos conversores multinível série e paralelo são analisados observando-se a variação de amplitude da corrente de entrada, que é controlada pelo controlador do elo CC.

Foi utilizada a fonte de alimentação alternada California Instruments, modelo 4500LX, para gerar as tensões de entrada do equipamento com formas de onda senoidais e distorcidas.

As formas de onda de corrente e de tensão foram abstraídas a partir de um osciloscópio digital Agilent MSOX3014A-ATO-9050, bem como os pontos das formas de onda, de forma a serem utilizadas em ambiente Matlab ${ }^{\circledR}$ para analisar os espectros harmônicos e a taxa de distorção harmônica.

\subsection{Descrição do Protótipo Experimental}

Nas figuras 7.1 e 7.2 são apresentadas as fotos do protótipo UPQC multínivel monofásico, implementado em laboratório, com a indicação das principais peças e componentes utilizados na construção contendo $76 \mathrm{~cm}$ de altura por $.70 \mathrm{~cm}$ de largura. 
Figura 7.1 - Foto do protótipo UPQC (parte da frente).

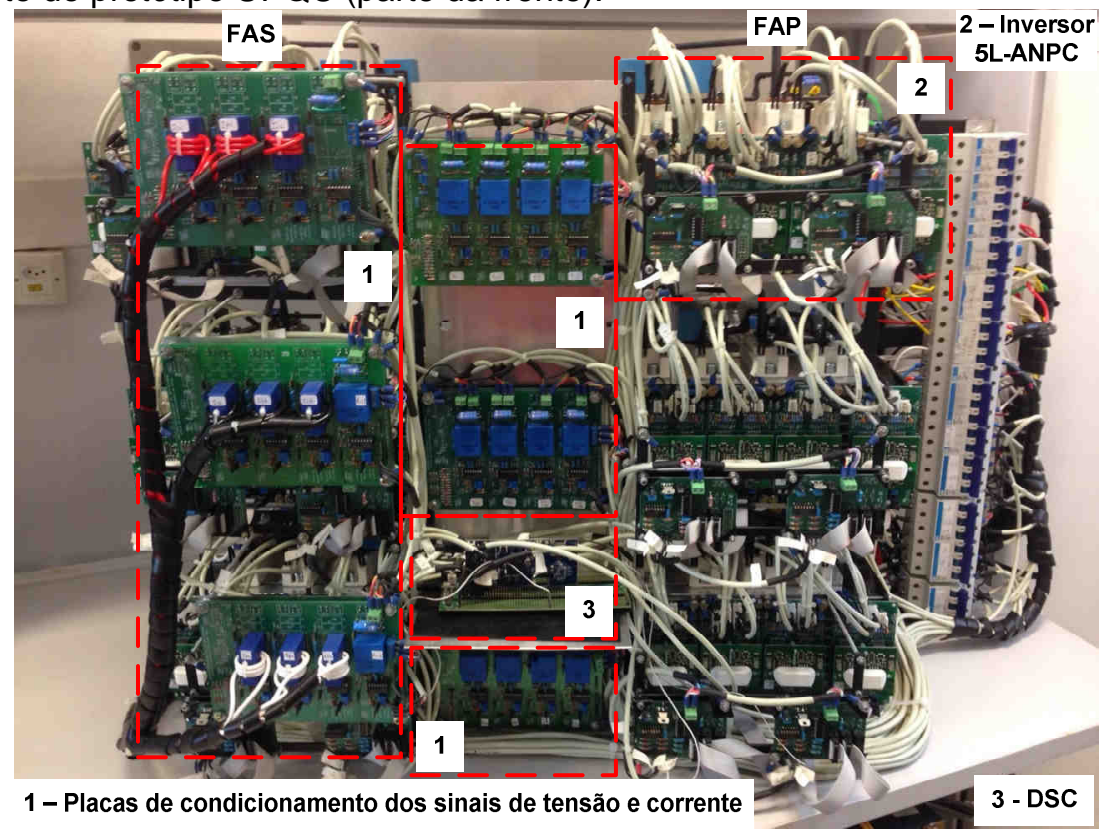

Fonte: Dados da pesquisa do autor.

Figura 7.2 - Foto do protótipo UPQC (parte de trás).

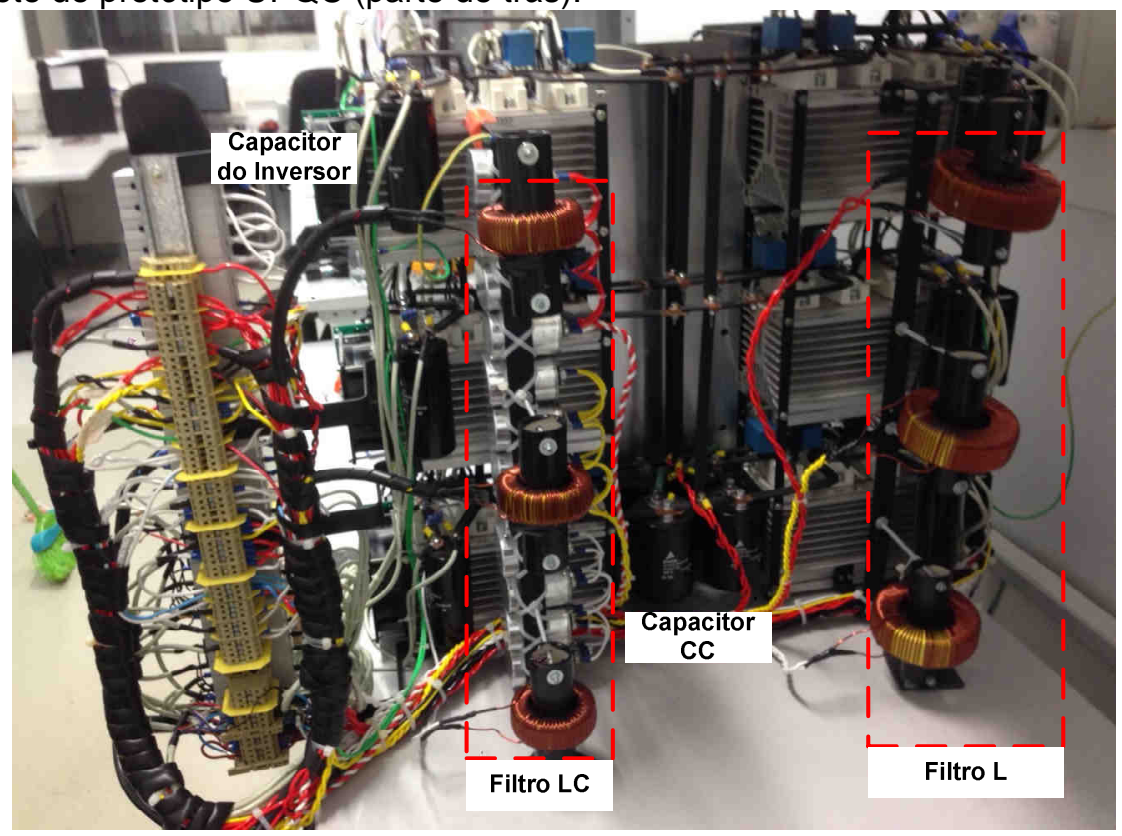

Fonte: Dados da pesquisa do autor.

Este protótipo consiste em seis módulos de inversores multíniveis $5 \mathrm{~L}-\mathrm{ANPC}$ independentes, em que nesta configuração foi separado três módulos para o FAS e três módulos para o FAP. Nesta aplicação, utilizou-se apenas dois módulos, ou seja, um módulo para FAS e outro para FAP. As características de cada inversor multínivel de potência são: 
- Dissipador Semikron P3 com 250mm 67247800;

- IGBT: 4 módulos de potência SKM75GB063D SEMITRANS 2 SUPERFAST NPT-IGBT, $V_{C E S}=600 \mathrm{~V}$, Icnom $=75 \mathrm{~A}$;

- Driver's: 4 peças PIMSKPC SKYPER 32/2 SEMITRANS 6R8-6R8-13 K-100PF;

- Driver's: 4 peças SKYPER 32R L6100102;

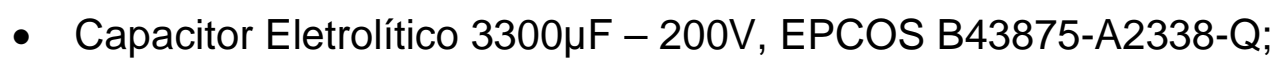

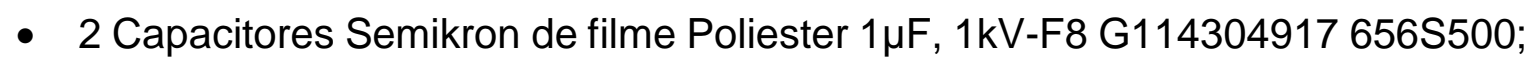

- Ventilador Semikron SK2120-AC-220V ROHS 67545100.

Já no inversor série de potência (FAS), são utilizados transformadores de acoplamento série e indutores de filtragem acoplados na rede elétrica. No inversor paralelo de potência são utilizados um acoplamento indutivo capacitivo conectado na carga. O elo CC é composto por 4 capacitores eletrolíticos modelo B43876-S9478-M1

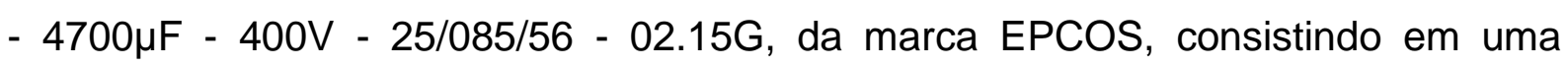
capacitância total de $9,3 \mathrm{mF}$.

Nas placas de condicionamento de sinais, são utilizados sensores de efeito hall modelo LV25-P - 715267 e LA100-P - 715172, para medições das tensões e correntes, ambos da marca LEM. Esses sensores realizam as seguintes medições: tensão da rede elétrica (circuito PLL), tensão do elo CC (controle do elo CC total e desequilíbrio), tensão de saída (controle da tensão na carga do conversor paralelo), tensão do capacitor de fase (capacitor do inversor multinível), corrente da carga (extração da componente fundamental de corrente da carga), corrente do indutor do filtro série (controle de corrente do conversor série) e corrente do indutor do filtro paralelo (malha interna de controle de corrente do conversor paralelo).

As medições dos sinais elétricos são condicionadas por meio de circuitos eletrônicos de forma a enviar esses sinais ao Controlador Digital de Sinais (DSC Digital Signal Controller) modelo TMDSDOCK28379D - SOPRANO F28379D Experimenter Kit, ponto flutuante com frequência de clock de $200 \mathrm{MHz}, 24$ entradas analógicas de 12 bits e 24 saídas PWM, da Texas Instruments. As malhas de controle do filtro ativo série e paralelo, elo e desequilíbrio CC, algoritmo PLL e CPT, foram implementados digitalmente no DSC. 
Na Figura 7.3, é mostrado o diagrama de blocos do protótipo desenvolvido.

Figura 7.3 - Diagrama de blocos do protótipo UPQC implementado em laboratório.

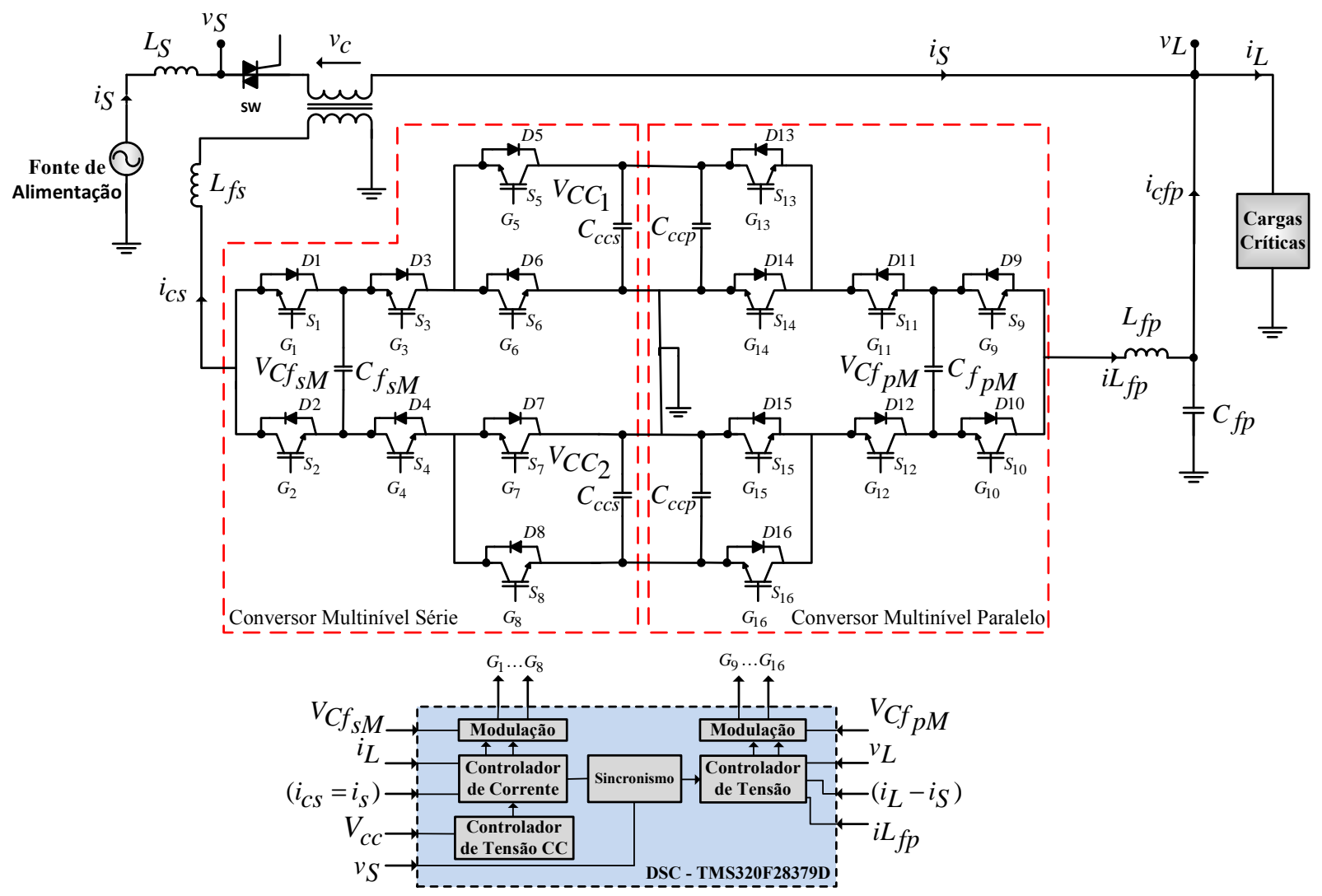

Fonte: Dados da pesquisa do autor.

Suas principais características estão descritas na tabela 7.1.

Tabela 7.1 - Parâmetros utilizados no experimento implementado.

\begin{tabular}{c|c}
\hline Tensão eficaz nominal da rede elétrica (fase-neutro) & $v_{S}=63,63 \mathrm{~V}$ \\
\hline Tensão eficaz nominal de saída (fase-neutro) & $v_{L}=63,63 \mathrm{~V}$ \\
\hline Percentual dos harmônicos na tensão de entrada & $v_{5}=6 \%, v_{7}=6 \%$ \\
\hline Tensão total do elo CC & $V_{c c}=250 \mathrm{~V}$ \\
\hline Tensão sobre o capacitor de fase (conversor Multinivel) & $V_{f}=62,5 \mathrm{~V}$ \\
\hline Frequência da rede elétrica & $f_{S}=60 \mathrm{~Hz}$ \\
\hline Frequência de chaveamento dos conversores multiniveis PWM série e & $f_{C}=20 \mathrm{kHz}$ \\
\hline paralelo & $f_{C}=40 \mathrm{kHz}$ \\
\hline Frequência de amostragem do conversor A/D - DSC & $L_{f s}=4,8 \mathrm{mH}$ \\
\hline Indutor de acoplamento do inversor 5L-ANPC série & $L_{f p}=1,3 \mathrm{mH}$ \\
\hline Indutor de acoplamento do inversor 5L-ANPC paralelo & $C_{f p}=80 \mu \mathrm{F}$ \\
\hline Capacitor de filtragem do inversor 5L-ANPC paralelo & $C_{f s}, p M=3300 \mu \mathrm{F}$ \\
\hline Capacitor de fase do inversor 5L-ANPC
\end{tabular}




\begin{tabular}{c|c}
\hline Capacitância total do elo CC & $C_{V c c T}=9400 \mu F$ \\
\hline Relação de transformação do transformador de acoplamento série & $n=1$ \\
\hline Potência do transformador de acoplamento série & $2 \mathrm{kVA}$ \\
\hline Potência nominal da carga & $1 \mathrm{kVA}$ \\
\hline Resistência da carga & $R=17 \Omega$ \\
\hline Indutância da carga & $L=280 \mathrm{mH}$ \\
\hline Capacitância da carga & $C=940 \mu \mathrm{F}$ \\
\hline
\end{tabular}

Fonte: Dados da pesquisa do autor.

\subsection{Resultados Experimentais}

As figuras 7.4 (a), (b) e (c) mostram as tensões e corrente do inversor 5L-ANPC de tensão, em malha aberta, com uma carga resistiva, ou seja, a tensão do capacitor de fase $V_{C f, M}$, tensão no inversor $V_{i n v}$, tensão de saída $V_{o}$ e corrente de fase do capacitor $I_{C f}$. Percebe-se que a tensão sobre o capacitor de fase $V_{C f, M}$ está controlada, com valor de $62,5 \mathrm{~V}$ e não sofre variações, assim como a tensão do inversor $V_{i n v}$ tem as características do cinco níveis de acordo com metodologia abordada no capítulo 3, assim como uma distorção harmônica total de 25,78\%.

Figura 7.4 - Inversor de tensão 5L-ANPC em malha aberta: (a) e (b) tensão do capacitor de fase $V_{C f, M}$ (100V/div, 5ms/div), tensão no inversor $V_{i n v}(100 \mathrm{~V} / \mathrm{div}, 5 \mathrm{~ms} / \mathrm{div})$, tensão de saída $V_{o}(100 \mathrm{~V} / \mathrm{div}, 5 \mathrm{~ms} / \mathrm{div})$ e corrente no capacitor de fase $I_{C f}(10 \mathrm{~A} / \mathrm{div}, 5 \mathrm{~ms} / \mathrm{div})$ e (c) tensão no inversor $V_{i n v}(100 \mathrm{~V} / \mathrm{div}, 5 \mathrm{~ms} / \mathrm{div})$, tensão de saída $V_{o}(100 \mathrm{~V} / \mathrm{div}, 5 \mathrm{~ms} / \mathrm{div})$ e corrente no capacitor de fase $I_{C f}(10 \mathrm{~A} / \mathrm{div}, 5 \mathrm{~ms} / \mathrm{div})$.

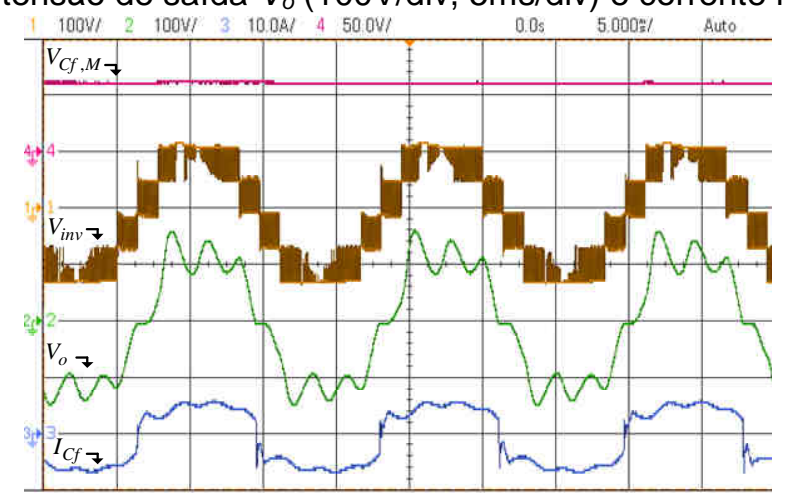

(a)

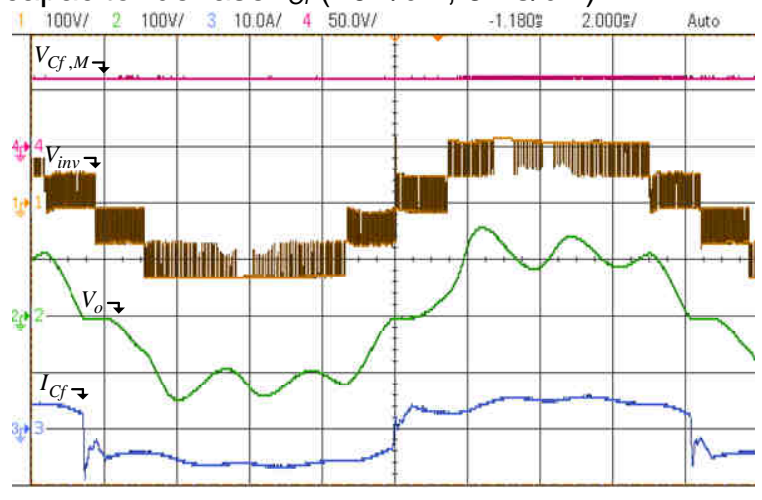

(b) 


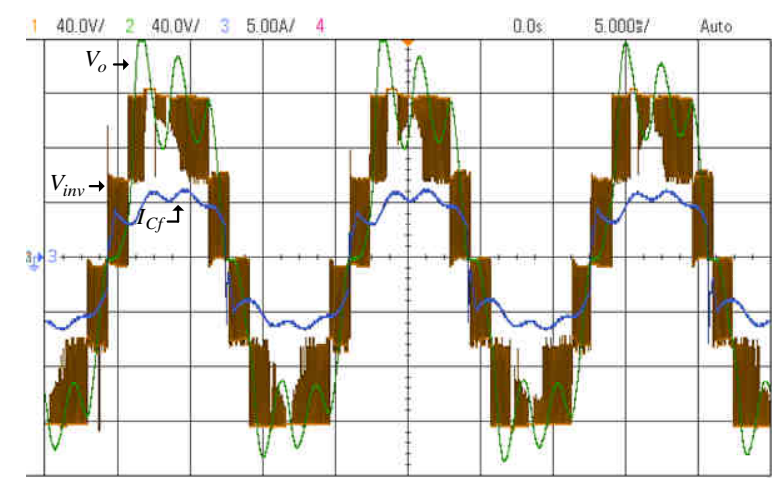

(c)

Fonte: Dados da pesquisa do autor.

As figuras 7.5 (a) e (b) mostram a tensão de saída e as correntes da UPQC com carga $R L$ e RC, ou seja, a tensão de saída do conversor paralelo $v_{L}$ (FAP), a corrente de entrada já compensada $i_{S}$, a corrente compensada fornecida pelo conversor paralelo $i_{C f p}$ e a corrente na carga não compensada $i_{L}$. Pode-se constatar que a corrente de entrada é senoidal e com baixo conteúdo harmônico, assim como para a tensão de saída, tanto para carga $\mathrm{RL}$ como $\mathrm{RC}$.

As figuras 7.6 (a) e (b) mostram as tensões e correntes da UPQC com carga $\mathrm{RL}$ e RC, ou seja, a tensão de entrada $v_{S}$, tensão de saída do conversor paralelo $v_{L}$ , a corrente de entrada já compensada $i_{S}$ e a corrente na carga $i_{L}$. Percebe-se que a tensão e corrente de entrada estão em fase, assim como a tensão e corrente de saída. Já as correntes de entrada compensadas com ambas as cargas $R L$ e RC possuem baixo conteúdo harmônico.

Figura 7.5 - UPQC: (a) tensão de saída $v_{L}(100 \mathrm{~V} / \mathrm{div}, 10 \mathrm{~ms} / \mathrm{div})$, corrente de entrada is $(5 \mathrm{~A} / \mathrm{div}$, $10 \mathrm{~ms} / \mathrm{div})$, corrente de compensação paralela $i_{c f p}(5 \mathrm{~A} / \mathrm{div}, 10 \mathrm{~ms} / \mathrm{div})$ e corrente de carga $-\mathrm{RL}$ i $(5 \mathrm{~A} / \mathrm{div}$, $10 \mathrm{~ms} / \mathrm{div}) ;$ (b) tensão de saída $v_{L}(100 \mathrm{~V} / \mathrm{div}, 5 \mathrm{~ms} / \mathrm{div})$, corrente de entrada is (5A/div, $\left.5 \mathrm{~ms} / \mathrm{div}\right)$, corrente de compensação paralela $i_{c f p}(5 \mathrm{~A} / \mathrm{div}, 5 \mathrm{~ms} / \mathrm{div})$ e corrente de carga $-\mathrm{RC} i \mathrm{~L}(5 \mathrm{~A} / \mathrm{div}, 5 \mathrm{~ms} / \mathrm{div})$.

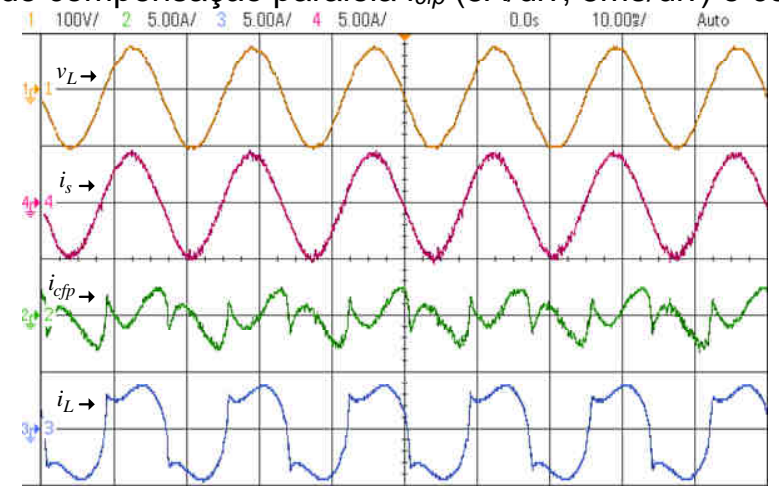

(a)

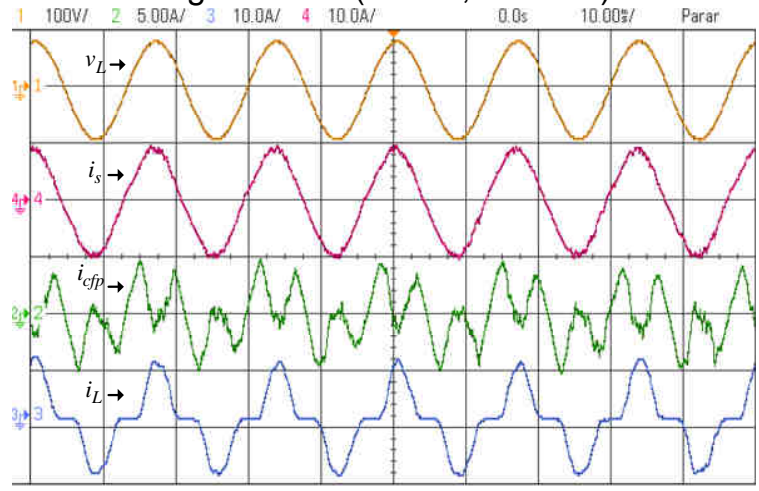

(b)

Fonte: Dados da pesquisa do autor. 
Figura 7.6 - UPQC: (a) tensão de entrada $v_{s}(50 \mathrm{~V} / \mathrm{div}, 10 \mathrm{~ms} / \mathrm{div})$, corrente de entrada is $(5 \mathrm{~A} / \mathrm{div}$, $10 \mathrm{~ms} / \mathrm{div})$, tensão de saída $v_{L}(50 \mathrm{~V} / \mathrm{div}, 10 \mathrm{~ms} / \mathrm{div})$, corrente de carga $-\mathrm{RL} i_{L}(5 \mathrm{~A} / \mathrm{div}, 10 \mathrm{~ms} / \mathrm{div}) ;(\mathrm{b})$ tensão de entrada $v_{S}(50 \mathrm{~V} / \mathrm{div}, 10 \mathrm{~ms} / \mathrm{div})$, corrente de entrada $i_{S}(5 \mathrm{~A} / \mathrm{div}, 10 \mathrm{~ms} / \mathrm{div})$, tensão de saída $v_{L}$ (50V/div, 10ms/div), corrente de carga $-R C i_{L}(5 \mathrm{~A} / \mathrm{div}, 10 \mathrm{~ms} / \mathrm{div})$.

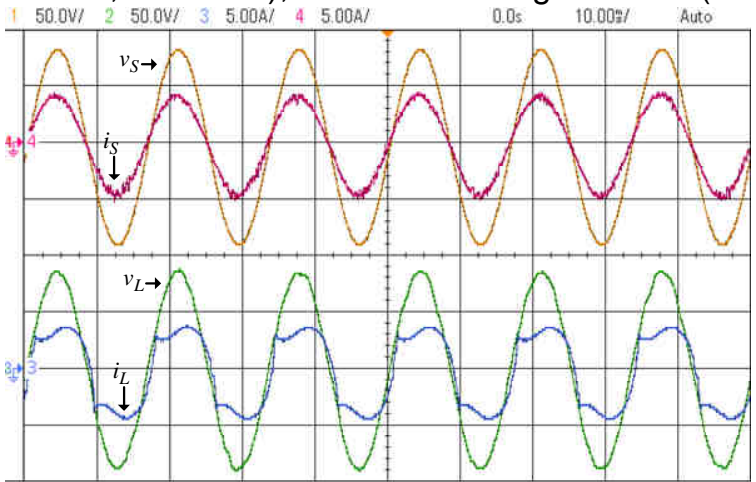

(a)

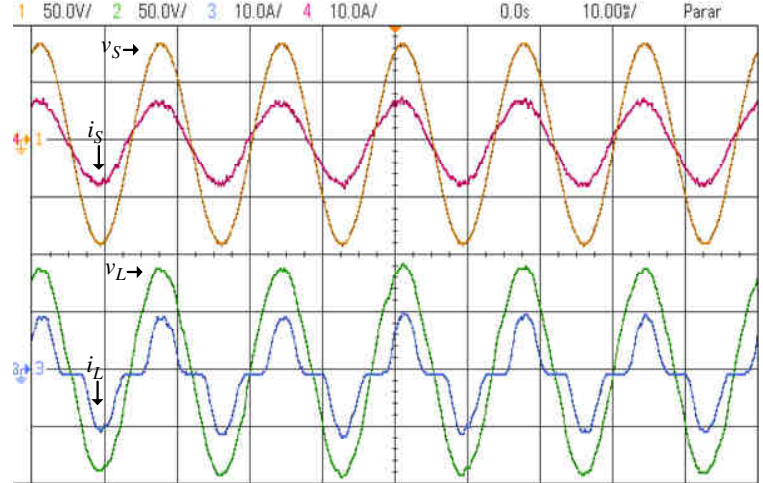

(b)

Fonte: Dados da pesquisa do autor.

As figuras 7.7 (a) e (b) mostram as tensões e correntes da UPQC com carga $\mathrm{RL}$ e RC, ou seja, a tensão de entrada $v_{S}$ distorcida, tensão de saída do conversor paralelo $v_{L}$, a corrente de entrada já compensada $i_{S}$ e a corrente na carga $i_{L}$. Percebe-se que a tensão e a corrente de entrada estão em fase, assim como a tensão e a corrente de saída. Já as correntes de entrada, compensadas tanto com cargas $R L$ e RC, possuem baixo conteúdo harmônico, ao passo que a tensão de entrada apresenta conteúdo harmônico.

Figura 7.7 - UPQC com conteúdo harmônico em $v_{S}$ : (a) tensão de entrada $v_{S}(50 \mathrm{~V} / \mathrm{div}, 10 \mathrm{~ms} / \mathrm{div})$, corrente de entrada $i_{S}(5 \mathrm{~A} / \mathrm{div}, 10 \mathrm{~ms} / \mathrm{div})$, tensão de saída $v_{L}(50 \mathrm{~V} / \mathrm{div}, 10 \mathrm{~ms} / \mathrm{div})$ e corrente de carga $\mathrm{RL} i_{L}(5 \mathrm{~A} / \mathrm{div}, 10 \mathrm{~ms} / \mathrm{div})$; (b) tensão de entrada $v_{S}(50 \mathrm{~V} / \mathrm{div}, 10 \mathrm{~ms} / \mathrm{div})$, corrente de entrada $i_{S}(10 \mathrm{~A} / \mathrm{div}$, $10 \mathrm{~ms} / \mathrm{div})$, tensão de saída $v_{L}(50 \mathrm{~V} / \mathrm{div}, 10 \mathrm{~ms} / \mathrm{div})$ e corrente de carga - RC $i_{L}(10 \mathrm{~A} / \mathrm{div}, 10 \mathrm{~ms} / \mathrm{div})$.

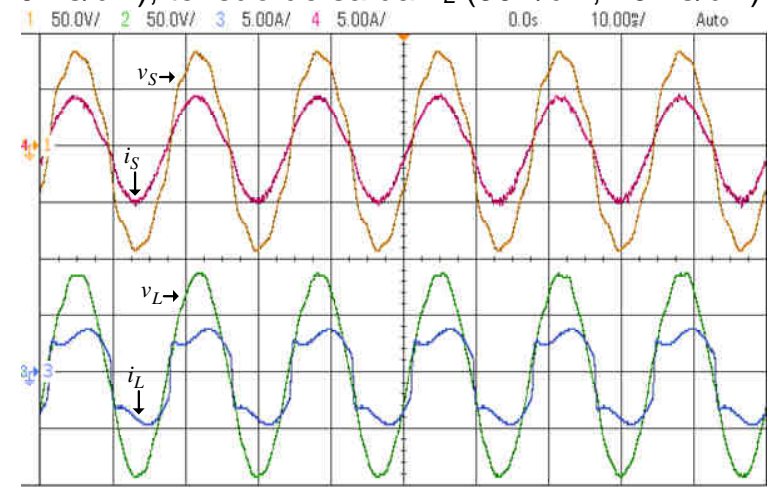

(a)

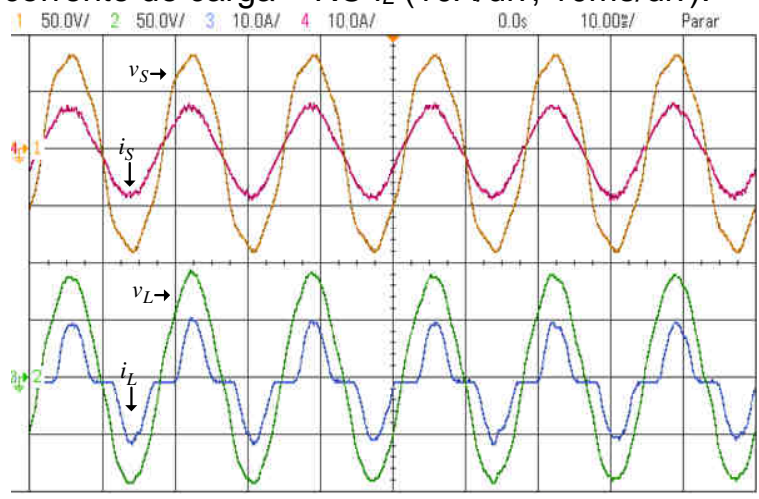

(b)

Fonte: Dados da pesquisa do autor. 
Na Figura 7.8, mostram-se a tensão de entrada $v_{S}$ em fase com a tensão de saída $v_{L}$ e a tensão sobre o transformador de acoplamento série $v_{C}$, que representa a diferença entre $v_{S}$ e $v_{L}$.

Figura 7.8 - UPQC: tensão de entrada $v_{S}(30 \mathrm{~V} / \mathrm{div}, 10 \mathrm{~ms} / \mathrm{div})$, tensão de saída $v_{L}(30 \mathrm{~V} / \mathrm{div}, 10 \mathrm{~ms} / \mathrm{div})$ e tensão sobre o transformador de acoplamento; $v_{C}(5 \mathrm{~V} / \mathrm{div}, 10 \mathrm{~ms} / \mathrm{div})$; carga RL.

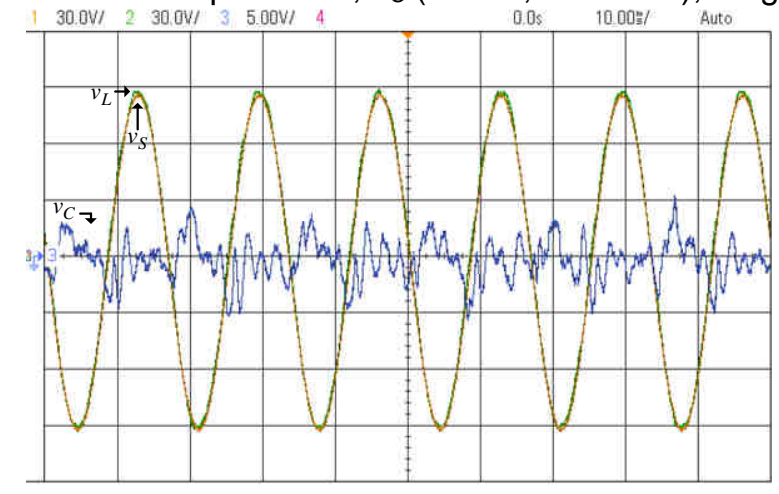

Fonte: Dados da pesquisa do autor.

Na Figura 7.9, mostram-se a tensão de entrada distorcida $v_{S}$ em fase com a tensão de saída $v_{L}$ e a tensão sobre o transformador de acoplamento série $v_{C}$, que representa a diferença entre $v_{S}$ e $v_{L}$.

Figura 7.9 - UPQC com conteúdo harmônico em $v_{S}$ : (a) tensão de entrada $v_{S}(30 \mathrm{~V} / \mathrm{div}, 10 \mathrm{~ms} / \mathrm{div})$, tensão de saída $v_{L}(30 \mathrm{~V} / \mathrm{div}, 10 \mathrm{~ms} / \mathrm{div})$ e tensão sobre o transformador de acoplamento; $v_{C}(20 \mathrm{~V} / \mathrm{div}$, $10 \mathrm{~ms} / \mathrm{div})$.

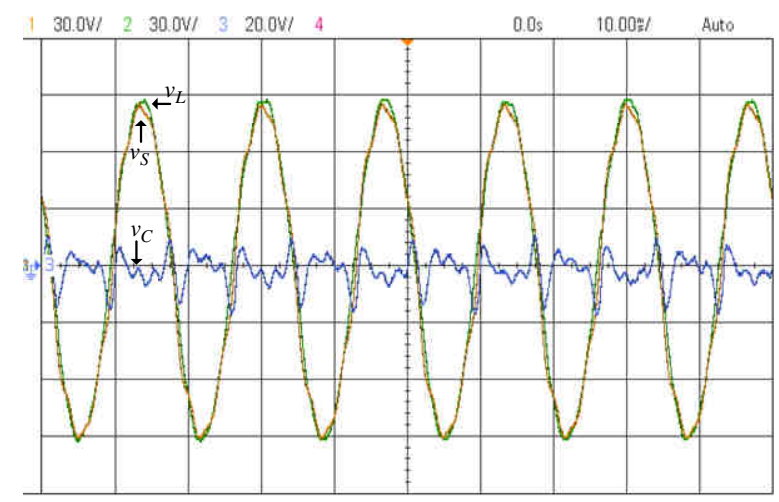

Fonte: Dados da pesquisa do autor.

Nas figuras 7.10 e 7.11, verifica-se a regulação da tensão de saída do UPQC mediante variações da tensão de entrada. A Figura 7.10 e 7.11 (a) mostra a tensão de entrada $v_{S}$, a tensão de saída $v_{L}$ e a tensão sobre o transformador de acoplamento série $v_{C}$, em que o valor eficaz da tensão de entrada $\left(v_{S}=72,45 \mathrm{~V}\right)$ é maior que a tensão de saída $\left(v_{L}=63,63 \mathrm{~V}\right)$, ou seja, $v_{S}>v_{L}$. A Figura 7.12 e 7.13 (b) mostra a 
tensão de entrada $v_{S}$, a tensão de saída $v_{L}$ e a tensão sobre o transformador de acoplamento série $v_{C}$, em que o valor eficaz da tensão de entrada $\left(v_{S}=54,08 \mathrm{~V}\right)$ é menor que a tensão de saída $\left(v_{L}=63,63 \mathrm{~V}\right)$, ou seja, $v_{S}<v_{L}$. Verifica-se que a tensão de saída sempre é regulada para um valor eficaz de 63,63V, independentemente do valor da tensão de entrada.

Percebe-se que a amplitude da corrente de entrada é diferente, quando se tem variações da tensão da entrada $v_{S}$ de acordo com o seu nível, ou seja, quando a tensão de entrada é maior ou menor que a tensão de saída, de acordo com as figuras 7.11 e 7.13, de forma a manter o fluxo de potência na UPQC.

Figura 7.10 - UPQC: (a) $\left(v_{S}>v_{L}\right)$ : tensão de entrada $v_{S}$ (30V/div, 10ms/div), tensão de saída $v_{L}$ (30V/div, $10 \mathrm{~ms} / \mathrm{div})$ e tensão sobre o transformador de acoplamento; $v_{C}(30 \mathrm{~V} / \mathrm{div}, 10 \mathrm{~ms} / \mathrm{div})$; (b) $\left(v_{S}<v_{L}\right)$ : tensão de entrada $v_{S}(30 \mathrm{~V} / \mathrm{div}, 10 \mathrm{~ms} / \mathrm{div})$, tensão de saída $v_{L}(30 \mathrm{~V} / \mathrm{div}, 10 \mathrm{~ms} / \mathrm{div})$ e tensão sobre 0 transformador de acoplamento; $v_{C}(30 \mathrm{~V} / \mathrm{div}, 10 \mathrm{~ms} / \mathrm{div})$.

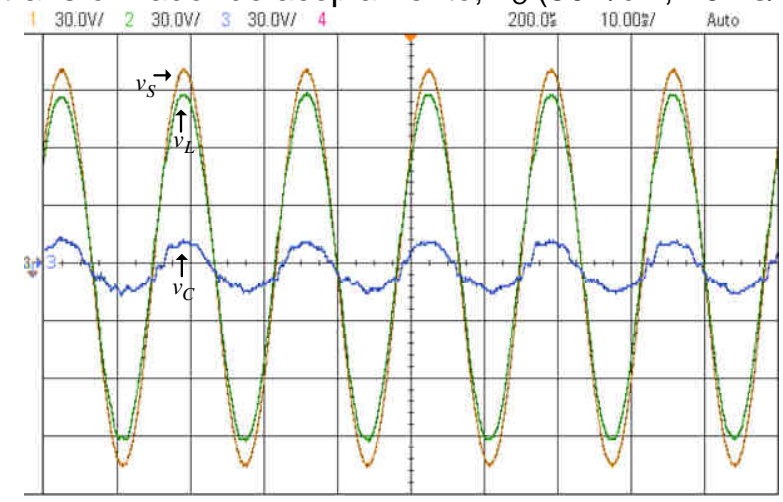

(a)

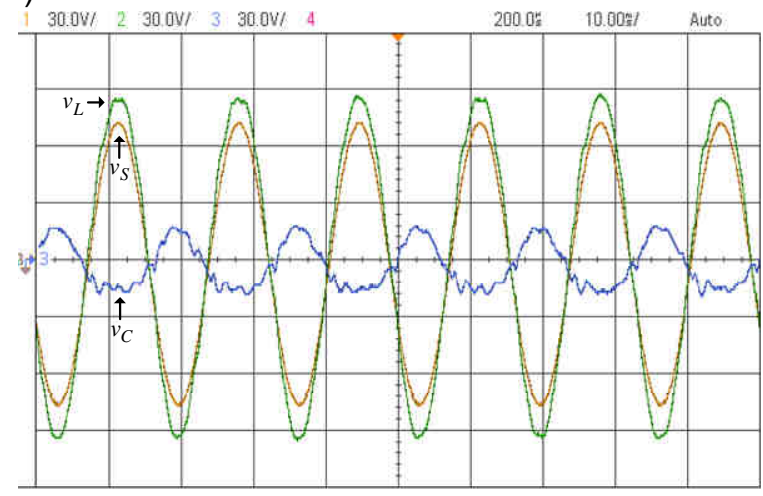

(b)

Fonte: Dados da pesquisa do autor.

Figura 7.11 - UPQC: (a) $\left(v_{S}>v_{L}\right)$ : tensão de entrada $v_{S}(30 \mathrm{~V} / \mathrm{div}, 10 \mathrm{~ms} / \mathrm{div})$, tensão de saída $v_{L}(30 \mathrm{~V} / \mathrm{div}$, $10 \mathrm{~ms} / \mathrm{div})$, corrente de entrada $i_{S}(5 \mathrm{~A} / \mathrm{div}, 10 \mathrm{~ms} / \mathrm{div})$ e corrente de carga $-\mathrm{RL} i_{L}(5 \mathrm{~A} / \mathrm{div}, 10 \mathrm{~ms} / \mathrm{div}) ;(\mathrm{b})$ $\left(v_{S}<v_{L}\right)$ : tensão de entrada $v_{S}(30 \mathrm{~V} / \mathrm{div}, 10 \mathrm{~ms} / \mathrm{div})$, tensão de saída $v_{L}(30 \mathrm{~V} / \mathrm{div}, 10 \mathrm{~ms} / \mathrm{div})$, corrente de entrada is (5A/div, $10 \mathrm{~ms} /$ div) e corrente de carga - RL $i_{L}(5 \mathrm{~A} / \mathrm{div}, 10 \mathrm{~ms} / \mathrm{div})$.

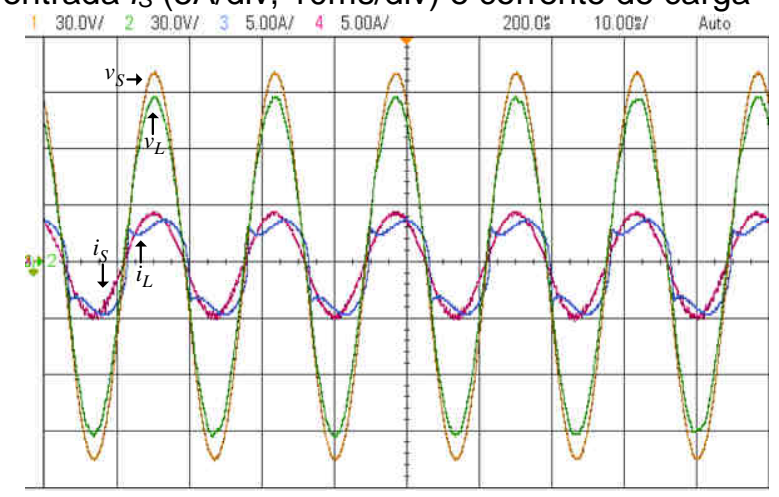

(a)

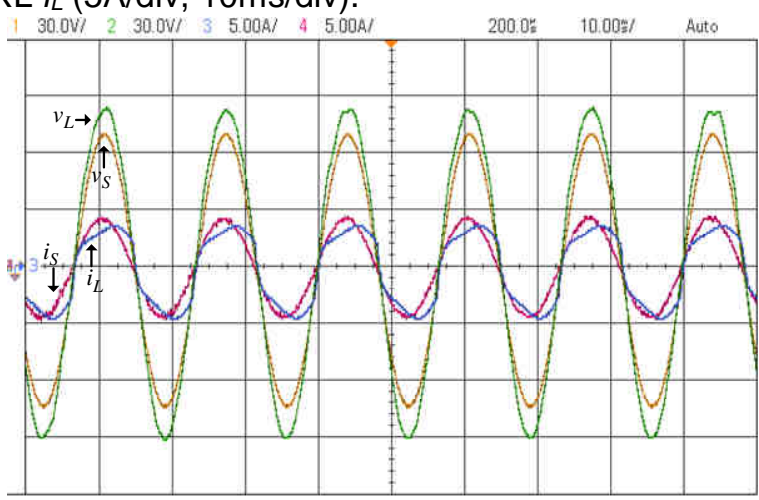

(b)

Fonte: Dados da pesquisa do autor. 
Nas figuras 7.12 e 7.13, verifica-se a regulação de tensão da saída do UPQC mediante variações da tensão de entrada com taxa de distorção harmônica. A Figura 7.12 e 7.13 (a) mostra a tensão de entrada $v_{S}$, tensão de saída $v_{L}$ e a tensão de acoplamento série $v_{C}$, em que o valor eficaz da tensão de entrada ( $v_{S}=72,45 \mathrm{~V}$ ) é maior que a tensão de saída $\left(v_{L}=63,63 \mathrm{~V}\right)$, ou seja, $v_{S}>v_{L}$. A Figura 7.12 e 7.13 (b) mostra a tensão de entrada $v_{S}$, tensão de saída $v_{L}$ e a tensão de acoplamento série $v_{C}$, em que o valor eficaz da tensão de entrada $\left(v_{S}=54,08 \mathrm{~V}\right)$ é menor que a tensão de saída $\left(v_{L}=63,63 \mathrm{~V}\right)$, ou seja, $v_{S}<v_{L}$. Verifica-se que a tensão de saída sempre é regulada para um valor eficaz de $63,63 \mathrm{~V}$, independentemente do valor da tensão de entrada.

Figura 7.12 - UPQC com conteúdo harmônico em $v_{S}$ : (a) $\left(v_{S}>v_{L}\right)$ : tensão de entrada $v_{S}(30 \mathrm{~V} / \mathrm{div}$, $10 \mathrm{~ms} / \mathrm{div})$, tensão de saída $v_{L}(30 \mathrm{~V} / \mathrm{div}, 10 \mathrm{~ms} / \mathrm{div})$ e tensão sobre o transformador de acoplamento; $v_{C}$ (30V/div, $10 \mathrm{~ms} / \mathrm{div}) ;$ (b) $\left(v_{S}<v_{L}\right)$ : tensão de entrada $v_{S}$ (30V/div, $\left.10 \mathrm{~ms} / \mathrm{div}\right)$, tensão de saída $v_{L}(30 \mathrm{~V} / \mathrm{div}$, $10 \mathrm{~ms} / \mathrm{div})$ e tensão sobre o transformador de acoplamento $v_{C}(30 \mathrm{~V} / \mathrm{div}, 10 \mathrm{~ms} / \mathrm{div})$.

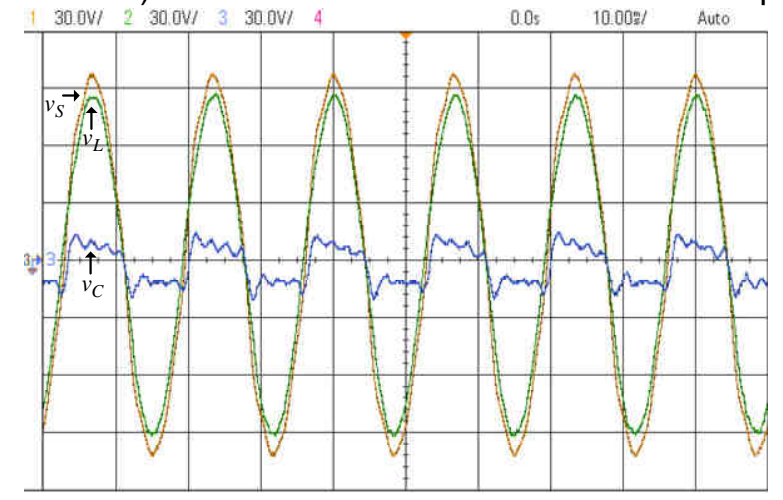

(a)

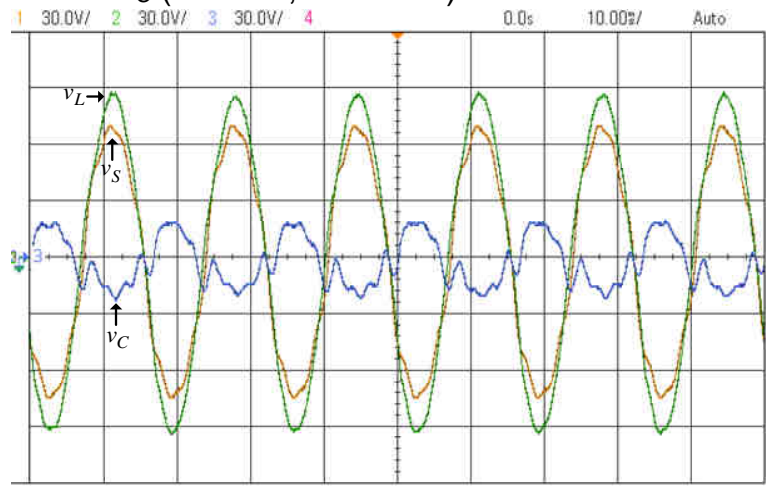

(b)

Fonte: Dados da pesquisa do autor. 
Figura 7.13 - UPQC com conteúdo harmônico em $v_{S}$ : (a) $\left(v_{S}>v_{L}\right)$ : tensão de entrada $v_{S}(30 \mathrm{~V} / \mathrm{div}$, $10 \mathrm{~ms} / \mathrm{div})$, tensão de saída $v_{L}(30 \mathrm{~V} / \mathrm{div}, 10 \mathrm{~ms} / \mathrm{div})$, corrente de entrada is $(5 \mathrm{~A} / \mathrm{div}, 10 \mathrm{~ms} / \mathrm{div})$ e corrente de carga - RL $i_{L}(5 \mathrm{~A} / \mathrm{div}, 10 \mathrm{~ms} / \mathrm{div})$; (b) $\left(v_{S}<v_{L}\right)$ : tensão de entrada $v_{S}(30 \mathrm{~V} / \mathrm{div}, 10 \mathrm{~ms} / \mathrm{div})$, tensão de saída $v_{L}(30 \mathrm{~V} / \mathrm{div}, 10 \mathrm{~ms} / \mathrm{div})$, corrente de entrada $i_{S}(5 \mathrm{~A} / \mathrm{div}, 10 \mathrm{~ms} / \mathrm{div})$ e corrente de carga $-\mathrm{RL} i_{L}$ (5A/div, 10ms/div).

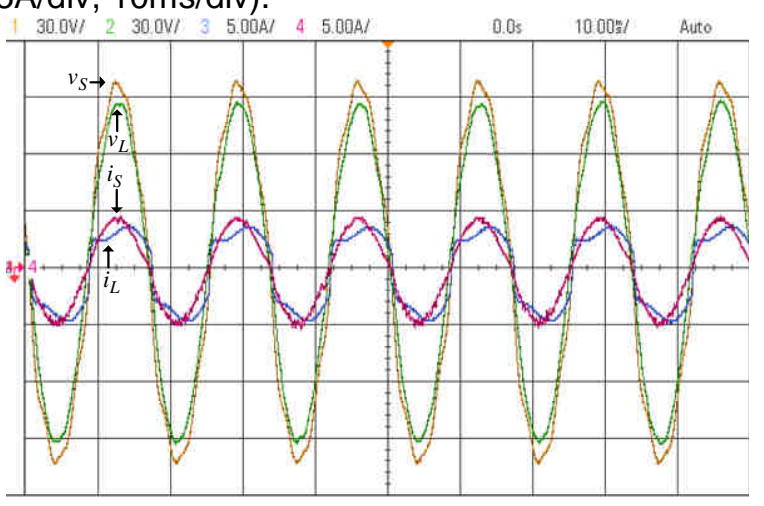

(a)

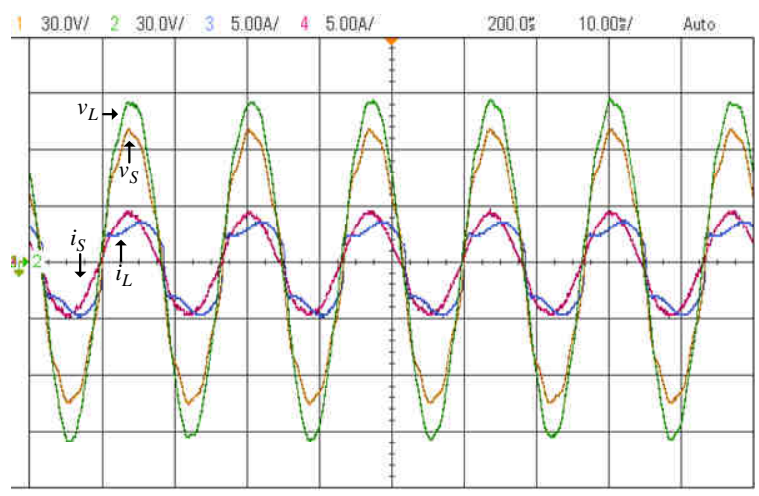

(b)

Fonte: Dados da pesquisa do autor.

As figuras 7.14, 7.15 e 7.16 (a) e (b) mostram o comportamento dinâmico de acordo com as variações de carga RL e RC no UPQC, de acordo com a tensão do capacitor de fase do inversor paralelo $V_{C f p, M}$, a tensão de entrada $v_{S}$, a tensão de saída $v_{L}$, a corrente de entrada já compensada $i_{S}$, a corrente compensada fornecida pelo conversor paralelo $i_{C f p}$ e a corrente na carga não compensada $i_{L}$. Nas figuras 7.14, 7.15 e 7.16 (a), a carga utilizada é $R L$ e, nas figuras 7.14, 7.15 e, 7.16 (b), a carga é RC. Dessa forma, são realizadas as variações de $50 \%$ a $100 \%$ e, em seguida, de $100 \%$ a $50 \%$ do seu valor nominal. Nota-se que o controle de corrente do inversor série e inversor de tensão foram capazes de controlar essas variações de modo que o sistema não foi a instabilidade. 
Figura 7.14 - UPQC com degrau de carga: (a) tensão do capacitor de fase $V_{C f}$ (10V/div, 200ms/div), corrente de entrada is ( $50 \mathrm{~A} / \mathrm{div}, 200 \mathrm{~ms} / \mathrm{div})$, corrente de compensação paralela $i_{c t p}(50 \mathrm{~A} / \mathrm{div}, 200 \mathrm{~ms} / \mathrm{div})$ e corrente de carga - RL $i_{L}(50 \mathrm{~A} / \mathrm{div}, 200 \mathrm{~ms} / \mathrm{div})$; (b) tensão do capacitor de fase $V_{C t, M}(50 \mathrm{~V} / \mathrm{div}$, $200 \mathrm{~ms} / \mathrm{div})$, corrente de entrada $i_{S}(20 \mathrm{~A} / \mathrm{div}, 200 \mathrm{~ms} / \mathrm{div})$, corrente de compensação paralela $i_{c f p}(20 \mathrm{~A} / \mathrm{div}$, $200 \mathrm{~ms} / \mathrm{div}$ ) e corrente de carga - RC $i_{L}$ (20A/div, 200ms/div).

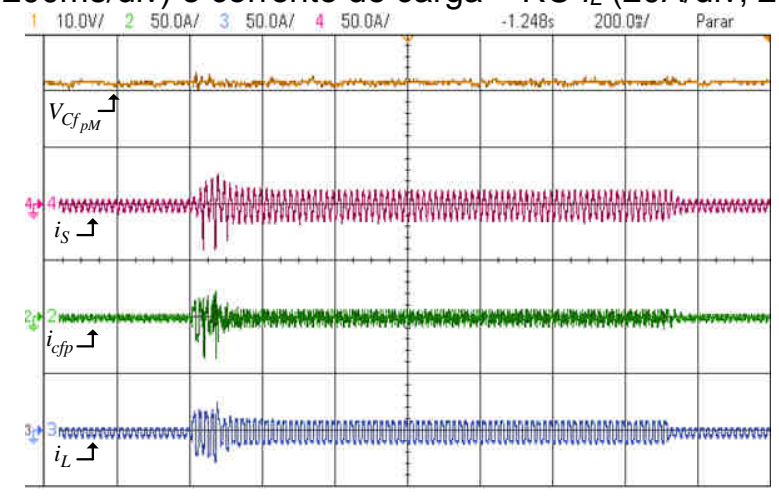

(a)

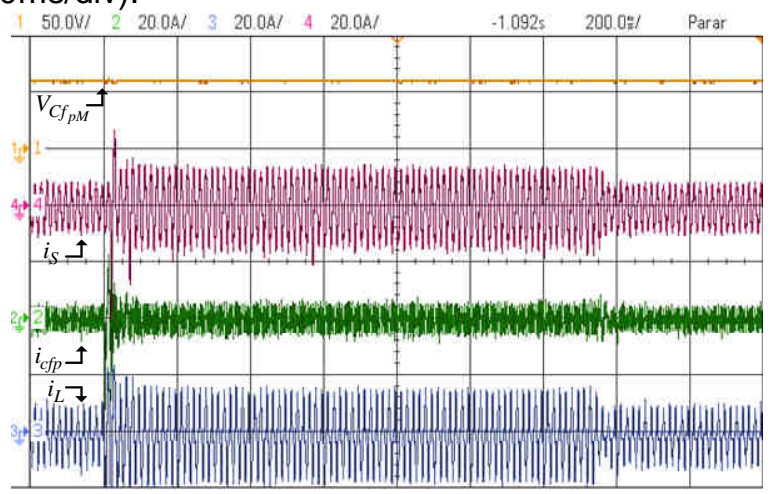

(b)

Fonte: Dados da pesquisa do autor.

Figura 7.15 - UPQC com degrau de carga: (a) tensão de entrada $v_{S}(100 \mathrm{~V} / \mathrm{div}, 200 \mathrm{~ms} / \mathrm{div})$, corrente de entrada is (50A/div, $200 \mathrm{~ms} / \mathrm{div})$, corrente de compensação paralela $i_{c f p}(50 \mathrm{~A} / \mathrm{div}, 200 \mathrm{~ms} / \mathrm{div})$ e corrente de carga - RL $i_{L}(50 \mathrm{~A} / \mathrm{div}, 200 \mathrm{~ms} / \mathrm{div})$; (b) tensão de entrada $v_{S}(100 \mathrm{~V} / \mathrm{div}, 200 \mathrm{~ms} / \mathrm{div})$, corrente de entrada is (20A/div, $200 \mathrm{~ms} / \mathrm{div})$, corrente de compensação paralela $i_{\text {cfp }}(20 \mathrm{~A} / \mathrm{div}, 200 \mathrm{~ms} / \mathrm{div})$ e corrente de carga $-\mathrm{RC} i_{L}(20 \mathrm{~A} / \mathrm{div}, 200 \mathrm{~ms} / \mathrm{div})$.

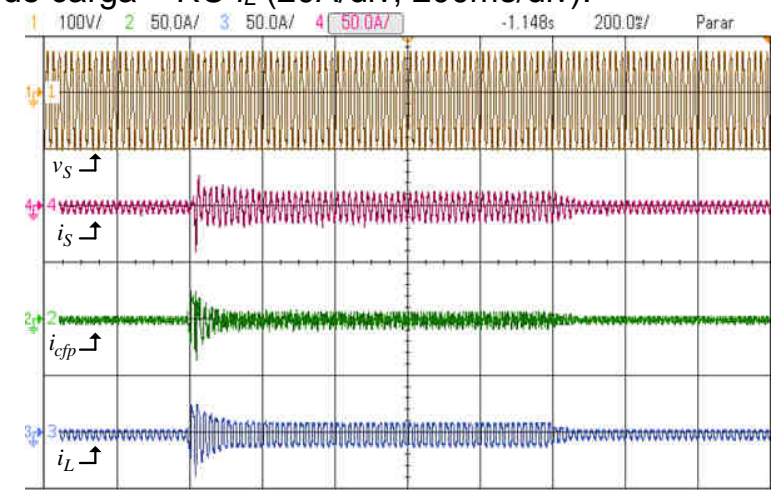

(a)

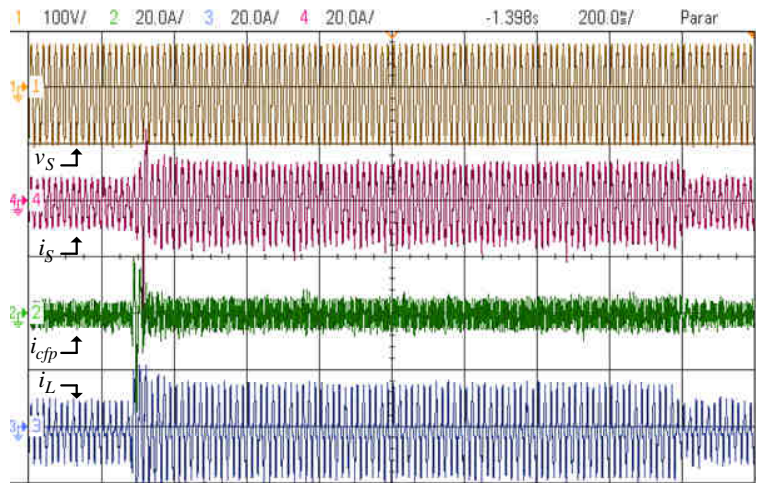

(b)

Fonte: Dados da pesquisa do autor. 
Figura 7.16 - UPQC com degrau de carga: (a) tensão de saída $v_{L}(100 \mathrm{~V} / \mathrm{div}, 200 \mathrm{~ms} / \mathrm{div})$, corrente de entrada is (50A/div, $200 \mathrm{~ms} / \mathrm{div})$, corrente de compensação paralela $i_{\text {cfp }}(50 \mathrm{~A} / \mathrm{div}, 200 \mathrm{~ms} / \mathrm{div})$ e corrente de carga - RL $i_{L}(50 \mathrm{~A} / \mathrm{div}, 200 \mathrm{~ms} / \mathrm{div})$; (b) tensão de saída $v_{L}(100 \mathrm{~V} / \mathrm{div}, 200 \mathrm{~ms} / \mathrm{div})$, corrente de entrada $i_{S}(20 \mathrm{~A} / \mathrm{div}, 200 \mathrm{~ms} / \mathrm{div})$, corrente de compensação paralela $i_{c f p}(20 \mathrm{~A} / \mathrm{div}, 200 \mathrm{~ms} / \mathrm{div})$ e corrente de carga $-\mathrm{RC} i_{L}$ (20A/div, 200ms/div).

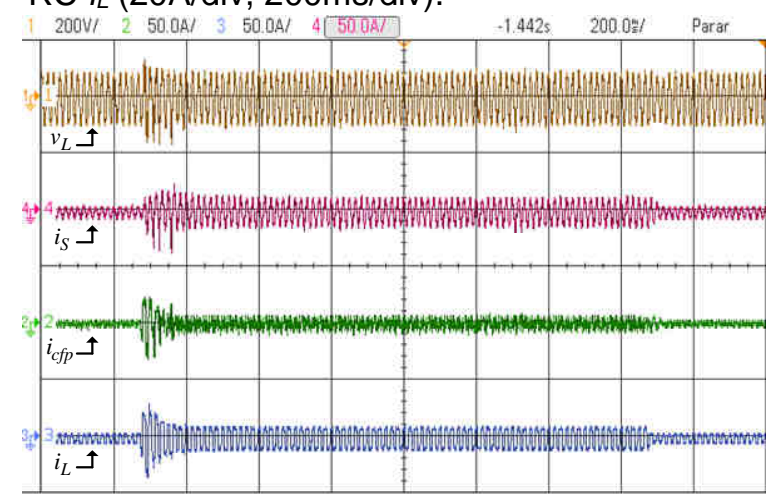

(a)

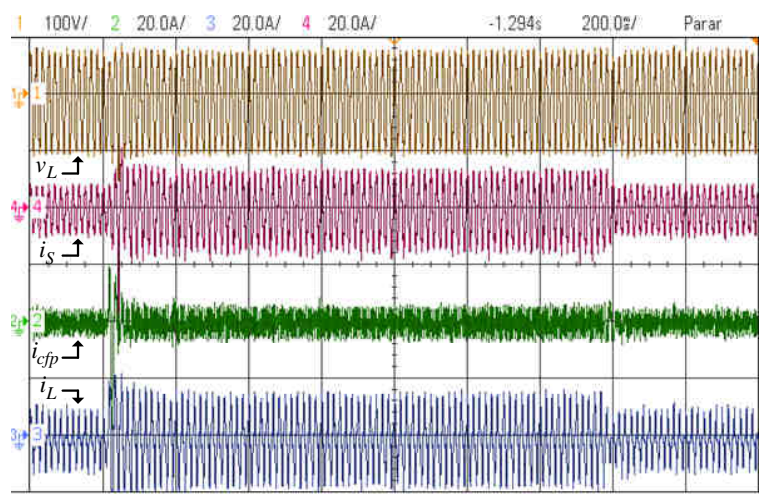

(b)

Fonte: Dados da pesquisa do autor.

A Figura 7.17 (a) e (b) mostra o comportamento dinâmico de acordo com as variações de carga RC na UPQC, conforme a tensão do elo CC total $V_{C C_{T}}$, a tensão dos elos superior $V_{C C_{1}}$ e inferior $V_{C C_{2}}$ e a corrente de entrada $i_{S}$. Na Figura 7.17 (a), varia-se a carga de $50 \%$ a $100 \%$ do seu valor nominal. Percebe-se uma redução nas tensões do elo CC e um aumento da amplitude da corrente de entrada. Já na Figura 7.17 (b), a carga é variada de $100 \%$ a $50 \%$ do seu valor nominal. Nota-se uma elevação nas tensões do elo CC e uma diminuição da corrente de entrada. Pode-se verificar que o controle de corrente do inversor série e do elo CC foram capazes de controlar essas variações de modo que o sistema não foi para a instabilidade.

Figura 7.17 - UPQC com degrau de carga - RC: (a) aumento de carga: tensão do elo total CC $\mathrm{V}_{C C T}$ (50V/div, $500 \mathrm{~ms} / \mathrm{div})$, tensão do elo superior $\mathrm{V}_{C C 1}(50 \mathrm{~V} / \mathrm{div}, 500 \mathrm{~ms} / \mathrm{div})$ e tensão do elo inferior $\mathrm{V}_{C C 2}$ (50V/div, 500ms/div); (b) retirada de carga: tensão do elo total $C C \mathrm{~V}_{C C T}(50 \mathrm{~V} / \mathrm{div}, 500 \mathrm{~ms} / \mathrm{div})$, tensão do elo superior $\mathrm{V}_{C c 1}$ (50V/div, $\left.500 \mathrm{~ms} / \mathrm{div}\right)$ e tensão do elo inferior $\mathrm{V}_{C c 2}(50 \mathrm{~V} / \mathrm{div}, 500 \mathrm{~ms} / \mathrm{div})$.

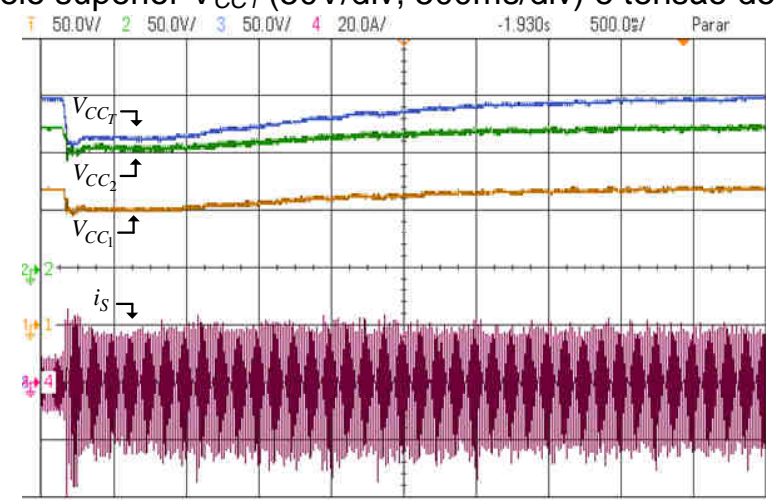

(a)

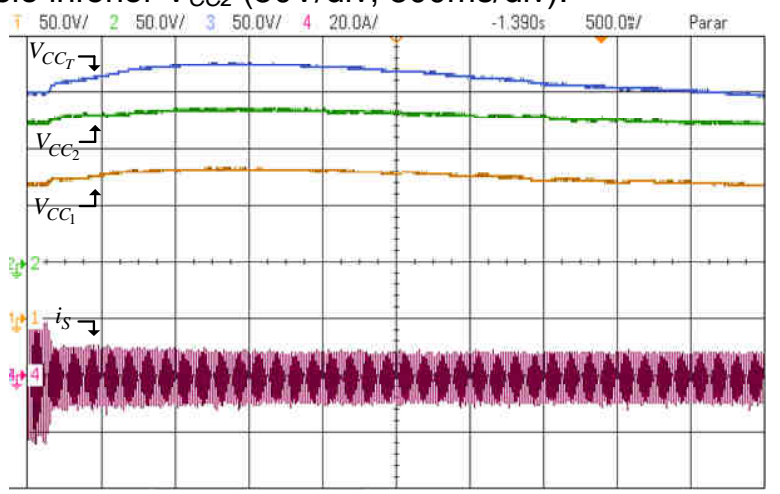

(b)

Fonte: Dados da pesquisa do autor. 
Na Figura 7.18, mostram-se a tensão do elo CC total $V_{C C_{T}}$, as tensões dos elos superior $V_{C C_{1}}$ e inferior $V_{C C_{2}}$ e a corrente de entrada $i_{S}$, quando ocorre um transitório de carga RC.

Figura 7.18 - UPQC com degrau de carga - RC: tensão do elo total CC $\mathrm{V}_{C C T}$ (50V/div, 1.4s/div), tensão do elo superior $\mathrm{V}_{C C 1}(50 \mathrm{~V} / \mathrm{div}, 1.4 \mathrm{~s} / \mathrm{div})$ e tensão do elo inferior $\mathrm{V}_{C c 2}(50 \mathrm{~V} / \mathrm{div}, 1.4 \mathrm{~s} / \mathrm{div})$

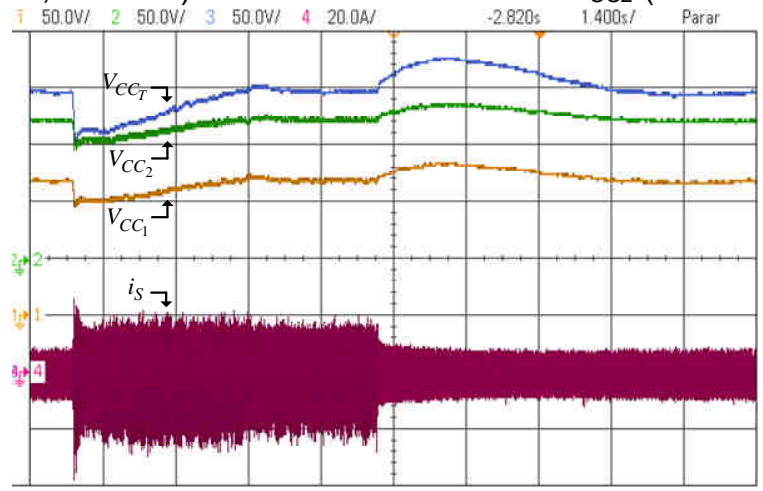

Fonte: Dados da pesquisa do autor.

Na Figura 7.19 (a), (b), (c) e (d), são apresentados os espectros harmônicos, como também a taxa de distorção harmônica total das tensões e das correntes do UPQC, considerando a tensão de entrada com DHT de 1,02\% e com carga RL. Por meio dos resultados encontrados, verifica-se uma redução na DHT da corrente de entrada, em que observa-se uma minimização de 29,4\% para 4,01\%. Em termos da tensão na carga, destaca-se que a DHT encontra-se em torno de $2,65 \%$, que está abaixo dos limites de $10 \%$, recomendado pelo PRODIST, e de $5 \%$, recomendado pela IEEE 519.

Figura 7.19 - Espectros harmônicos - carga RL.

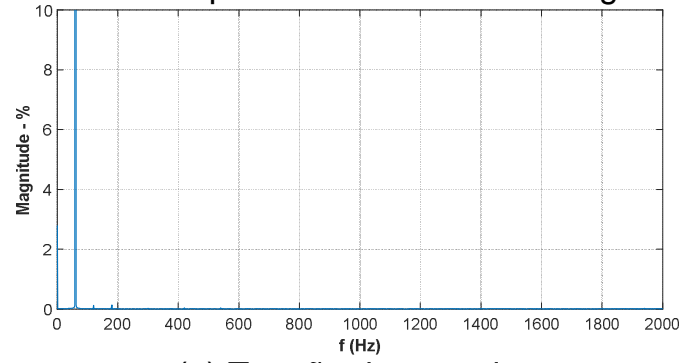

(a) Tensão de entrada

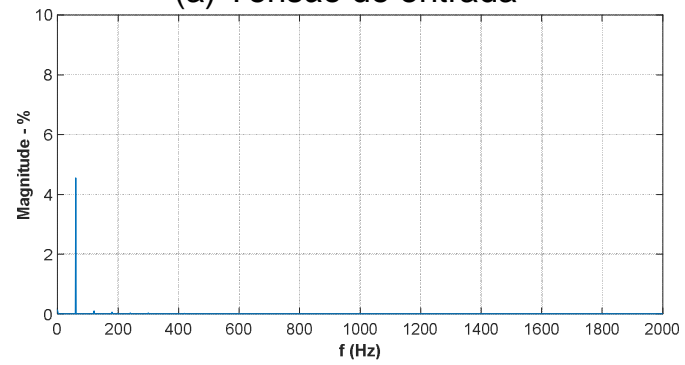

(c) Corrente de entrada

Fonte: Dados da pesquisa do autor.

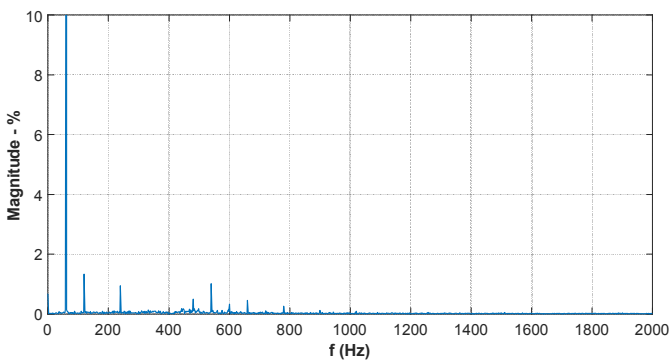

(b) Tensão de saída

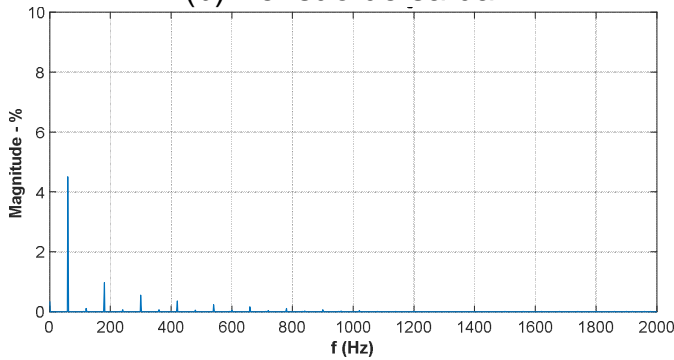

(d) Corrente de saída 
Na Figura 7.20 (a), (b), (c) e (d), também são apresentados os espectros harmônicos, como também a taxa de distorção harmônica total das tensões e das correntes do UPQC, considerando a tensão de entrada com DHT de 8,49\% e com carga RC. Por meio dos resultados encontrados, verifica-se uma redução na DHT da corrente de entrada, em que observa-se uma minimização de 47,29\% para 4,59\%. Em termos da tensão na carga, destaca-se que a DHT encontra-se em torno de $3,90 \%$, que está abaixo dos limites de $10 \%$, recomendado pelo PRODIST, e de $5 \%$, recomendado pela IEEE 519.

Figura 7.20 - Espectros harmônicos com tensão de entrada distorcida e carga RC.

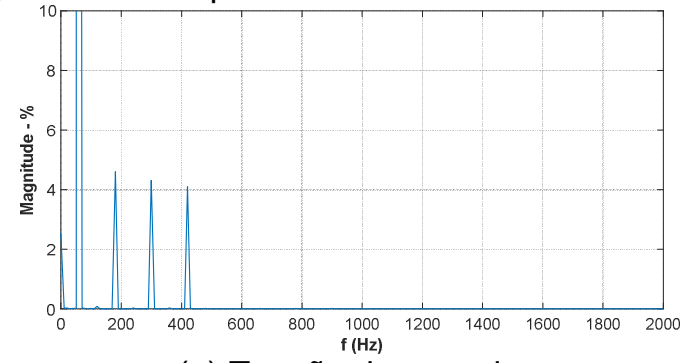

(a) Tensão de entrada

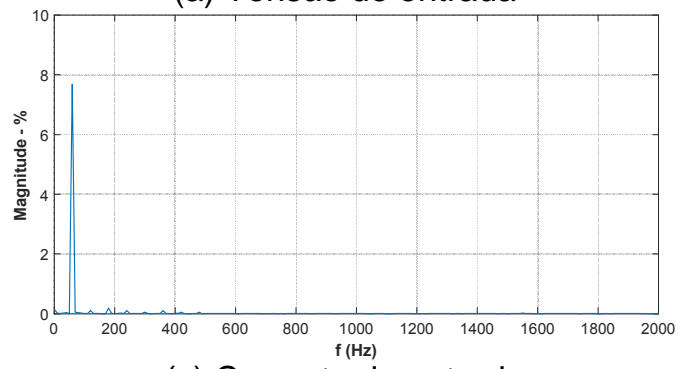

(c) Corrente de entrada

Fonte: Dados da pesquisa do autor.

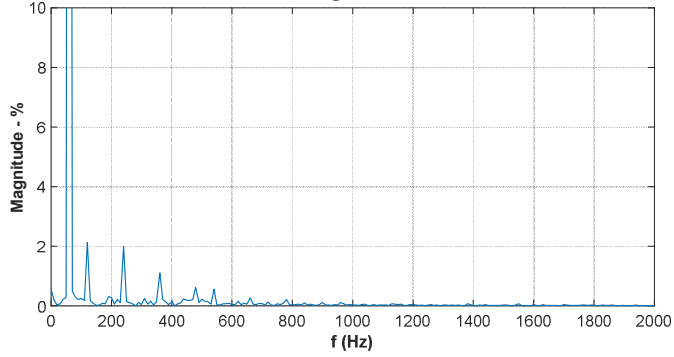

(b) Tensão de saída

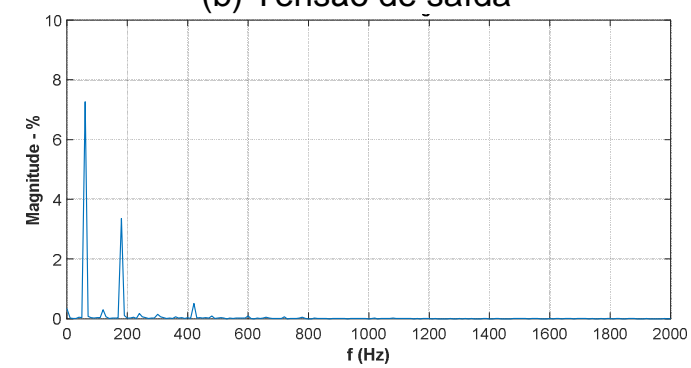

(d) Corrente de saída

\subsection{Considerações finais do capítulo}

Neste capítulo, foram apresentados os resultados por meio de ensaios experimentais obtidos a partir de um protótipo UPQC multinível monofásico com compensação ativa de potência série e paralela, de forma a validar todo o desenvolvimento teórico apresentado.

Os ensaios foram realizados, considerando-se a utilização de uma carga não linear, constituída por um retificador em ponte completa a diodos com carga RL e RC.

Foram apresentados os comportamentos estáticos e dinâmicos do UPQC, que mostraram desempenho satisfatório mediante a presença de distúrbios da rede de alimentação, tais como elevações e afundamentos de tensão, incidência de 
harmônicos, bem como degraus de carga. Dessa forma o UPQC foi capaz de impor corrente senoidal e tensão senoidal e regulada, com correção do fator de potência, além da eliminação de harmônicos.

O comportamento dinâmico dos controladores de tensão do elo CC foi avaliado sob variações de carga, em que foi utilizado dois controladores, um para o controle total do elo CC, e outro para controle dos desequilíbrios de tensão do elo CC. Desse modo, foi possível se observar a eficácia de ambos os controladores, visto que eles se mantêm constantes e balanceados nos casos avaliados.

O fluxo de potência por meio dos conversores série e paralelo foram verificados observando-se a variação de amplitude da corrente de entrada, que é controlada pelo controlador do elo CC.

Os resultados experimentais adquiridos mostraram tensões reguladas, com baixa taxa de conteúdo harmônico, corrente senoidal, com baixo conteúdo harmônico em fase com a tensão de entrada, resultando em elevado fator de potência.

As distorções harmônicas totais da tensão de saída e de corrente de entrada foram analisadas e comparadas com a recomendação IEEE 519 e a norma PRODIST. 


\section{Conclusões Gerais e Propostas de Continuidade}

\subsection{Conclusões Gerais}

Este trabalho apresentou o estudo, a análise, o controle e a implementação experimental de um Condicionador Unificado de Qualidade de Energia (UPQC) monofásico, ainda não presentes na literatura segundo a abordagem utilizada. Dessa forma, explorou-se o método de compensação ativa de potência, série e paralela, de modo que o seu princípio de funcionamento é descrito de forma generalizada, além das principais características em termos da estratégia de controle dual empregada na compensação ativa de potência.

De uma forma específica, é apresentado o princípio de funcionamento do inversor ANPC 5 níveis, em conjunto com o modelo matemático teórico e chaveado, a modulação $P H$, também conhecida como $L S$, e o fluxograma de implementação da modulação em microcontrolador de ponto flutuante.

Além disso, foi apresentada a Teoria da Potência Conservativa, de forma geral para tensões e correntes, e a definição de termos de potência e de energia por fase, em condições senoidais, não senoidais e/ou assimétricas, desenvolvida no domínio do tempo e aplicada para a rede de distribuição senoidal, não-senoidal, balanceada e desbalanceada. Em seguida, utilizou-se a CPT de forma a extrair somente a componente fundamental da corrente da carga, aplicada no conversor série de potência como algoritmo de geração de referência de corrente.

Em seguida, foram apresentadas as modelagens matemáticas das plantas dos conversores estáticos de potência multiníveis série e paralelo, do elo CC e do desequilíbrio do elo CC, bem como dos modelos dos controladores. Na sequência, foram realizados os projetos dos controladores a partir da margem de fase e de frequência de cruzamento.

Foram apresentados os estudos sobre o fluxo de potência do UPQC, que ocorrem por meio dos conversores série e paralelo. As análises foram feitas para diferentes características, todas mostradas por meio das curvas normalizadas das potências aparentes dos conversores.

Como forma de comprovar a capacidade dos conversores de compensação ativa de potência, realizou-se a análise de estabilidade das malhas de controle do 
conversor série de corrente e do conversor paralelo de tensão, para distúrbios envolvendo variações da corrente da carga, bem como variações da tensão da rede elétrica. A partir desta análise, comprovou-se que o dispositivo é estável para os respectivos ganhos dos controladores de ambos os conversores de potência.

Para a validação da proposta, foram apresentados os resultados de simulação do UPQC monofásico, em que as simulações computacionais foram realizadas por meio do software PSIM.

Os comportamentos estáticos e dinâmicos foram mostrados com a presença de distúrbios da rede elétrica, tais como harmônicos, afundamentos e elevações de tensões e degraus de carga.

De acordo com os aspectos construtivos e definidos, os modos de operação do UPQC, para ambos os conversores, sendo a estratégia de compensação dual, notase que a tensão de saída sempre será senoidal, regulada e com baixa taxa de distorção harmônica. Dessa maneira, os harmônicos, afundamentos e elevações de tensão, bem como perturbações da rede elétrica, sempre serão absorvidos pelo transformador de acoplamento série.

Sendo assim, observou-se que o transformador do conversor série de potência irá absorver de forma natural essas perturbações, por estar entre as tensões de entrada e de saída, que são indiretamente compensadas. Com isso, mostrou-se que não há necessidade de calcular qualquer referência de tensão de compensação para o conversor série de potência.

Como forma de verificar a aplicabilidade da proposta, foram apresentados os resultados por meio de ensaios experimentais obtidos a partir de um protótipo UPQC multinível monofásico com compensação ativa de potência série e paralela, de forma a validar todo o desenvolvimento teórico apresentado.

Os ensaios foram realizados considerando-se a utilização de uma carga não linear, constituída por um retificador em ponte completa a diodos com carga RL e RC. Foram apresentados os comportamentos estáticos e dinâmicos do UPQC, que mostraram desempenho satisfatório mediante a presença de distúrbios da rede de alimentação, tais como elevações e afundamentos de tensão, incidência de harmônicos, bem como degraus de carga. Dessa forma, o UPQC foi capaz de impor corrente senoidal e tensão senoidal e regulada, com correção do fator de potência, além da eliminação de harmônicos. 
O comportamento dinâmico dos controladores de tensão do elo $\mathrm{CC}$ foi avaliado sob variações de carga, em que foram utilizados dois controladores, um para o controle total do elo CC, e outro para controle dos desequilíbrios de tensão do elo CC. Desse modo, foi possível se observar a eficácia de ambos os controladores, visto que eles se mantêm constantes e balanceados nos casos avaliados.

O fluxo de potência por meio dos conversores série e paralelo foram verificados observando-se a variação de amplitude da corrente de entrada, que é controlada pelo controlador do elo CC.

Os resultados experimentais mostraram tensões reguladas, com baixa taxa de conteúdo harmônico, corrente senoidal, com baixo conteúdo harmônico em fase com a tensão de entrada, resultando em elevado fator de potência.

O sistema UPQC proposto realizou de forma correta as reduções das taxas de distorções harmônicas na corrente de entrada, bem como na tensão de saída, realizando a supressão dos harmônicos. Além disso, observou-se que os valores obtidos das taxas totais de distorções harmônicas ficaram dentro das recomendações PRODIST do IEEE 519.

Com o intuito de eliminar diversos tipos de problemas relacionados a Qualidade de Energia, O UPQC implementado neste trabalho demonstrou sua eficiência a partir dos resultados obtidos por simulação, e confirmados nos resultados experimentais, além de mostrar que as estratégias de controle e algoritmos de compensação utilizados são apropriadas para este tipo de aplicação, contribuindo assim para eliminação dos distúrbios na rede elétrica.

\subsection{Propostas de Continuidade}

A seguir as propostas de continuidade deste trabalho:

- Implementar o UPQC multinível ANPC cinco níveis em um sistema trifásico a quatro fios;

- Implementar o sistema de pré-carga dos capacitores do elo CC, de forma a realizar a partida do UPQC;

- A partir da topologia implementada, estudar os efeitos das interferências eletromagnéticas conduzidas e propor a aplicação de métodos de filtragens; 
- Estudar e implementar outros tipos de controladores para ambos os conversores série e paralelo, bem como para as tensões do elo CC como os controladores inteligentes e preditivos;

- Implementar a compensação seletiva de harmônicos na corrente de entrada do UPQC utilizando sistemas inteligentes, como, por exemplo, por meio da utilização de Redes Neurais Artificiais;

- Estudar e propor algoritmos de detecção de ilhamento de forma a definir procedimentos seguros para a desconexão do sistema UPQC durante uma interrupção no sistema de fornecimento de energia elétrica;

- Adicionar ao elo CC um sistema de geração de energia elétrica por meio de um sistema fotovoltaico de forma a injetar potência ativa na rede elétrica;

- Estudar e propor implementações do sistema UPQC em ambientes de microredes $\mathrm{CA}$.

\subsection{Publicações}

Durante o período deste trabalho, os seguintes artigos foram publicados:

- Rodrigo, B. Edson, J. A.; Ricardo, Q. M. Selective Compensation of Harmonics Applied in a Multi-Level Single-Phase UPQC System. IEEE 13th Brazilian Power Electronics Conference and 1st Southern Power Electronics Conference (COBEP/SPEC), Fortaleza, 2015.

- Edson, J. A.; Rodrigo, B.; Ricardo, Q. M. Five Level ANPC Converter Applied as a Single-Phase Shunt Active Power Filter With Features to Limit and Optimize the Converter Power Rating. IEEE 13th Brazilian Power Electronics Conference and 1st Southern Power Electronics Conference (COBEP/SPEC), Fortaleza, 2015.

- Rodrigo, B.; Rodrigo, A. M.; Augusto, S. P.; Edson, J. A.; Rodolpho, V. A. N.; Ricardo, Q. M. Compensação Seletiva de Harmônicos utilizando Redes 
Neurais Artificiais Aplicada em um Filtro Ativo de Potência Paralelo Multinível. Simpósio Brasileiro de Automação Inteligente (SBAI), Porto Alegre, 2017.

- Rodrigo, B. Edson, J. A.; Ricardo, Q. M. Filtro Ativo de Potência Paralelo Monofásico ANPC 5 Níveis Utilizando Redes Neurais como Método de Compensação Seletiva de Harmônicos. XII Conferência Brasileira sobre Qualidade de Energia Elétrica (CBQEE), Curitiba, 2017.

- Edson, J. A.; Rodrigo, B.; Ricardo, Q. M. Filtro Ativo Paralelo Multinível Avaliado Sob a IEEE STD 1459-2010. XII Conferência Brasileira sobre Qualidade de Energia Elétrica (CBQEE), Curitiba, 2017.

Artigos foram submetidos em periódicos e estão em fase de análise e avaliação. 


\section{Referências}

Angélico, B. A.; Campanhol, L. B. G.; Silva, S. A. O., Proportional-integral/proportionalintegral-derivative tuning procedure of a single-phase shunt active power filter using Bode diagram. IET Power Electronics, 2014, pp. 1-13.

Aredes, M.; Fernandes, R. M. A dual topology of unified power quality conditioner: the iUPQC. In: International European Power Electronics Conference, 13., Rio de Janeiro, 2009. Proceedings... Rio de Janeiro: IEEE, 2009. p. 1-10.

Aredes, M.; Fernandes, R. M. A Unified Power Quality Conditioner with Voltage Sag/Swell Compensation Capability. In: Brazilian Power Electronics Conference, 10., 2009, Rio de Janeiro. Proceedings... Rio de Janeiro: IEEE, 2009. p. 218-224.

Aredes, M.; Ferreira, D. A. C.; Siqueira, M. J. V.; França, B. W.; Silva, L. F.; Gerencer, R.. Performance Analysis and Technical Feasibility of an iUPQC in Industrial Grids. In: Journal of Power and Energy Engineering, v. 2, p. 500-508, 2014.

Aredes, M.; Häfner, J.; Huemann, K. A Combined Series and Shunt Active Power Filter. In: IEEE/KTH Stockholm Power Tech Conference, 1995, Stockholm. Proceedings... Stockholm: IEEE, 1995. p. 237-242.

Aredes, M.; Heumann, K.; Watanab, E. H.. An Universal Active Power Line Conditioner. In: IEEE Transaction on Power Delivery, v. 13, n. 2, p. 545-551, 1998.

Backer, R. H.; Bedford M. Switching Circuit. United States Patent, n. 4210826, 1980.

Backer, R. H.; Bedford, M. Bridge Converter Circuit. United States Patent, n. 4270163, 1979.

Baker R. H.; Bannister L. H. Electric Power Converter. U. S. Patent Number 3867643, Feb 1975.

BARBI, I. Projetos de fontes chaveadas. Florianópolis: Ed. do Autor, 2001. 
Barbosa, P.; Steimer, P.; Steinke, J.; Winkelnkemper, M.; Celanovic, N. Active-neutralpoint-clamped (ANPC) multilevel converter technology. In: Proc. IEEE Power Electron. Spec. Conf., Recife, Brasil, p. 10, Jun. 2005.

Barriviera, R.; Silva, S. A. O.; Kaster, M.; Modesto, R. A. Implementação de um Sistema UPS Monofásico com Condicionamento Ativo de Potência Série e Paralelo. Eletrônica de Potência (Impresso), v. 17, p. 753-762, 2012.

Barriviera, R.; Silva, S. A. O.; Kaster, M.; Modesto, R. A. Single-phase ups system with series-parallel filtering capabilities. In: Power Electronics Conference (COBEP), Natal, Brazil, p. 844-849, 2011.

Ben-Brahim, L.; Tadakuma, S. A novel multilevel carrier-based PWM-control method for GTO inverter in low index modulation region. In: IEEE Trans. Ind. Appl., vol. 42, no. 1, pp. 121-127, Jan./Feb. 2006.

Bhattacharya, S. et al. Parallel Active Filter System Implementation and Design Issues for Utility Interface of Adjustable Speed Drive Systems. In: Industry Applications Society Annual Meeting, 31., 1996, San Diego. Proceedings... San Diego: IEEE, 1996. p. 1032-1039.

Bhattacharya, S.; Divan, D. M.; Banerjee, B. Synchronous Frame Harmonic Isolator Using Active Series Filter. In: EPE, v. 3, p.30-35, 1991.

Blaadjerg, F.; He, J.; Li, Y. W.; Wang, X. Active harmonic filtering using currentcontrolled, grid-connected DG units with closed-loop power control. IEEE Transactions on Power Electronics 29, 2 (Feb. 2014).

Black, H. S. Modulation Theory. New York: D. Van Nostrand, 1953.

Blondel, A. Measurement of Energy of Polyphase Currents. In: III Proc. International Electrical Congress, Chicago, USA., pp. 112-117, 1893.

Bose, B. K. Power Electronics, Smart Grid, and Renewable Energy Systems. Proceeding of the IEEE. v. 105, №. 11, November 2017. 
Bouhali, O.; Francois, B.; Berkouk, E. M.; Saudemont, C. DC link capacitor voltage balancing in a three-phase diode clamped inverter controlled by a direct space vector of line-to-line voltages. In: IEEE Trans. Power Electron., vol. 22, no. 5, pp. 16361648, Sep. 2007.

Bowes, S. R. New sinusoidal pulsewidth modulated inverter. In: Proc. IEE, vol. 122, no. $11,1975$.

Brückner, T.; Bernet, S. Loss balancing in three-level voltage source inverters applying active NPC switches. In: $32^{\text {rd }}$ Annual IEEE Power Electronics Specialist Conference PESC, Canada, vol. 2, pp. 1135-1140, 2001.

Brückner, T.; Bernet, S.; Güldner, H. The active NPC converter and its loss-balancing control. In: IEEE Transactions on Industrial Electronics, vol. 52, no. 3, pp. 855868, June 2005.

Brückner, T.; Bernet, S.; Steimer, P. K. Feedforward loss control of three-level active NPC converters. In: IEEE Trans. Ind. Appl., vol. 43, no. 6, p. 1588-1596, Nov/Dec 2007.

Buso, S.; Mattavelli, P. Digital Control in Power Electronics - Synthesis Lectures on Power Electronics. [S.I.]: Morgan and Claypool Publishers, 2006. 1-158 p.

Campanhol, L. B. G. Sistema Fotovoltaico Trifásico de Único Estágio Conectado a Sistemas de Geração Distribuída Operando como Condicionador de Qualidade de Energia Unificado Usando uma Estratégia Dual de Compensação Ativa de Potência. Tese (Doutorado) - Escola de Engenharia de São Carlos da Universidade de São Paulo (EESC - USP), 2017.

Campoccia, A.; Cocchiara, G.; Ippolito, M. G.; et al. Fault decoupling device: a new device to reduce the impact of distributed generation on electrical distribution systems. IEEE Bologna Power Tech Conference Proceedings, vol. 4, pp. 7, 2003. 
Carrara, G.; et al. A New Multilevel PWM Method: A Theoretical Analysis. IEEE Transactions on Power Electronics, v. 7, no 3, p. 497-505, jul 1992.

Cataliotti, A. et al. Applications of the Fault Decoupling Device to Improve the Operation of LV Distribution Networks. IEEE Transactions on Power Delivery, v. 23, n. 1, p. 328-337, jan. 2008.

Chaitusaney, S.; Yokoyama, A. Impact of protection coordination on sizes of several distributed generation sources. The 7th International Power Engineering Conference, 2005. IPEC 2005. : 669-674 Vol. 2.

Choi, N. S.; Cho, J. G.; Cho G. H. A general circuit topology of multilevel inverter. In: IEEE Power Electronics Specialists Conference, PESC'91, p. 96-103, Jun. 1991.

Colak, I.; Kabalci, E.; Bayindir, R. Review of multilevel voltage source inverter topologies and control scheme. In: ELSEVIER, Energy Conversion and Management, p. 114-1128, 2010.

Dias, J.; Busarello, T. D. C.; Michels, L.; Reach, C.; Mezabora, M. "Controle Digital Simplificado para um Condicionador Unificado de Qualidade de Energia”, Revista Eletrônica de Potência, vol. 16, no. 3, pp. 212-221, 2011.

Distribution System Analysis Subcommittee IEEE, Radial Distribution Test Feeders, in PES Summer Meeting, 2000.

Dugan, R. C.; Mcdermott, T. E. Distributed generation: Operating conflicts for distributed generation interconnected with utility distribution systems. IEEE Industry Applications Magazine, vol. 8, pp. 19-25, 2002.

Dugan, R. C.; Mcgranaghan, M. F.; Beaty, H. W. Electrical power systems quality. New York: McGraw-Hill, 1996.

El-Khattam, W.; Salama, M. M. A. Distributed generation technologies, definitions and benefits. Electric Power Systems Research, vol. 71, pp. 119-128, 2004. 
Fitzgerald, A. E.; Jr., C. K.; Umans, S. D. Máquinas Elétricas. 6. ed. Porto Alegre: Bookman, 2006.

Floricau, D.; Floricau, E.; Dumitrescu, M. Natural doubling of the apparent switching frequency using three-level ANPC converter. In Proc. ISNCC Conf. Rec., Lágow, Poland, pp. 1-6, Jun. 2008.

Floricau, D.; Floricau, E.; Gateau, G. Three-level active NPC converter: PWM strategies and loss distribution. In Proc. IEEE IECON, pp. 3333-3338, Nov. 2008.

Franquelo, L. G.; Rodriguez, J.; Leon, J. I.; Portillo, S. K. R.; Prats, M. The age of Multilevel Converters arrives. In: IEEE Industrial Electronics Magazine, v. 2, n. 2, p. 28-39, Jun 2008.

Freijedo, F. et al. Frequency tracking of digital resonant filters for control of power converters connected to public distribution systems. IET Power Electronics, v. 4, n. 4, p. 454, 2011.

Fryze, S. Wirk-, Blind- und Scheinleistung in Elektrischen Stromkreisen mit Nichtsinusförmigem Verlauf von Strom und Spannung. ETZ-A Elektrotech Zeitschrift, vol. 53, no. 25, pp. 596-599, 625-627, 700-702, 1932.

Fujita, H; Akagi, H. The Unified Power Quality Conditioner: The Integration of Series Active Filters and Shunt Active Filters. In: Power Electronics Specialists Conference. Proceedings... Baveno: IEEE. v. 1, p. 494-501, 1996.

Gazoli, J. R. Microinversor Monofásico para Sistema Solar Fotovoltaico Conectado à Rede Elétrica. (Dissertação de mestrado) - Universidade Estadual de Campinas (UNICAMP), 2011.

Ghias, A. M. Y. M.; Pou, J.; Agelidis, V. G.; Ciobotaru, M. Voltage Balancing Method for a Flying Capacitor Multilevel Converter Using Phase Disposition PWM. In: IEEE Transactions on Industrial Electronics, vol. 61, no. 12, dec, 2014. 
GRIGOLETTO, F. B.; PINHEIRO, H. Método de modulação PWM para equilíbrio das tensões dos capacitores do barramento cc em conversores multiníveis com diodos de grampeamento. In: Revista Eletrônica de Potência - SOBRAEP, v. 14, n. 2, p. 6374, Maio 2009.

$\mathrm{He}$, J. et al. Active Harmonic Filtering Using Current-Controlled, Grid-Connected DG Units With Closed-Loop Power Control. IEEE Transactions on Power Electronics, v. 29 , n. 2, p. 642-653, fev. 2014.

Holmes, D. G.; Lipo, T. A. Pulse Width Modulation For Power Converters. Principles and Practice. IEEE Series on Power Enginnering, 2003. ISBN 0-471-20814-0.

Holtz J.; Oikonomou, N. Neutral point potential balancing algorithm at low modulation index for three-level inverter medium-voltage drives. In: IEEE Trans. Ind. Appl., vol. 43, no. 3, pp. 761-768, May/Jun. 2007.

Jeon, S. J.; Cho, G. H. A Series-Parallel Compensated Uninterruptible Power Supply with Sinusoidal Input Current and Sinusoidal Output Voltage. In: IEEE Power Electronics Specialists Conference, 28., St. Louis. Proceedings... St. Louis: IEEE, p. 297-303, 1997.

Jeon, S. J.; Cho, G. H. A Series-Parallel Compensated Uninterruptible Power Supply with Sinusoidal Input Current and Sinusoidal Output Voltage. In: $28^{\text {th }}$ IEEE Power Electronics Specialists Conference, p. 297-303, 1997.

Jin, B.; Yuan, X. Control of a Four-Level Active Neutral Point Clamped Converter with Neutral Point Voltage Balance. In: IEEE $8^{\text {th }}$ International Power Electronics and Motion Control Conference (IPEMC-ECCE Asia), 2016.

Kamran, F.; Habetler, T. "A novel on-line UPS with universal filtering capabilities," IEEE Transactions on Industrial Electronics, vol. 13, no. 2, pp. 366-371, 1998. 
Kamran, F.; Thomas, G.; Habetler. A Novel On-line UPS with Universal Filtering Capabilities. In: IEEE/PESC Power Electronics Specialists Conference, Atlanta, p500-506, 1995.

Karanki, K.; Geddada, G.; Mishra, M. K.; Kumar, B. K. A Modified Three-Phase FourWire UPQC Topology with Reduced DC-Link Voltage Rating. In: Transaction Industrial Electronics, v. 9, n. 60, p. 3555-3566, 2013.

Khadkikar, V. Enhancing Electric Power Quality using UPQC: A comprehensive overview. In: IEEE Transaction Power Electronics, v.27, n. 5, p. 2284-2297, 2012.

Khadkikar, V.; Chandra, A. A Novel Structure for Three-Phase Four-Wire Distribution System utilizing Unified Power Quality Conditioner (UPQC). In: IEEE Transaction on Industry Applications, v. 45, n. 5, p. 1897-1902, 2009.

Khadkikar, V.; Chandra, A.; Barry, A. O.; Nguyen, T. D. Power Quality Enhancement Utilising Single-Phase Unified Power Quality Conditioner: Digital Signal ProcessorBased Experimental Validation. In: IET Power Electronics, v. 4, iss. 3, p. 323-331, 2010.

Kim, J. H.; Sul, S.K.; Enjeti, P. N. A carrier-based PWM method with optimal switching sequence for a multilevel four-leg voltage-source inverter. In: IEEE Trans. Ind. Appl., vol. 44, no. 4, pp. 1239-1248, Jul./Aug. 2008.

Kwon, B.H.; Choi, J.H.; Kim, T.W. Improved single-phase line interactive UPS. IEEE Trans. on Industrial Electronics, Pohang, v. 48, n. 4, p. 804-811, 2001.

Lai, J. S.; Peng, F. Z. Multilevel converters - A new breed of Power converters. In: IEEE Transactions on Industry Applications, v. 32, p. 509-517, May/June 1996.

Lau, W. H.; Zhou, B.; Chung, H. S. H. Compact Analytical Solutions for Determining the Spectral Characteristics of Multicarrier-Based Multilevel PWM. In: IEEE Transactions on Circuits and Systems, vol. 51, no. 8, Aug. 2004. 
Li, J.; Huang, A. Q.; Bhattacharya, S.; Tan, G. Three-level active neutral-point-clamped (ANPC) converter with fault tolerant ability. In Proc. IEEE APEC, pp. 840-845, Feb. 2009.

Lima, L. R.; Novaes, Y. R.; Mezabora, M. Inversor de Tensão Monofásico NPC três níveis com Snubber Regenerativo. In: Eletrônica de Potência, Campo Grande, v. 16, n. 4, p. 320-329, Set/Nov 2011.

Liserre, M.; Teodorescu, R.; Blaabjerg, F. Multiple harmonics control for three-phase grid converter systems with the use of PI-RES current controller in a rotating frame. IEEE Transactions on Power Electronics, v. 21, n. 3, p. 836-841, maio 2006.

Loh, P. C.; Blaabjerg, F.; Gao, F.; Baby, A.; Tan, D. A. C. Pulsewidth modulation of neutral-point-clamped indirect matrix converter. In: IEEE Trans. Ind. Appl., vol. 44, no. 6, pp. 1805-1814, Nov./Dec. 2008.

Loh, P. C.; Blaabjerg, F.; Wong, C. P. Comparative evaluation of pulsewidth modulation strategies for Z-source neutral-point-clamped inverter. In: IEEE Trans. Power Electron., vol. 22, no. 3, pp. 1005-1013, May 2007.

Machado, R. Q. Sistema de Geração Distribuída com Fontes CA e CC Conectados a Rede Monofásica e Controle Eletrônico da Qualidade da Energia Elétrica. Tese (Tese de Doutorado) - Universidade Estadual de Campinas (UNICAMP), 2005.

Malinowski, M.; Stynski, S.; Kolomyjski, W.; Kazmierkowski, M. P. Control of threelevel PWM converter applied to variable-speed-type turbines. In: IEEE Trans. Ind. Electron., vol. 56, no. 1, pp. 69-77, Jan. 2009.

Marafão, F. B. Análise e controle da energia elétrica através de técnicas de processamento digital de sinais. Tese (Doutorado em Engenharia Elétrica) Departamento de Sistemas e Controle de Energia, Faculdade de Engenharia Elétrica e de Computação, Universidade Estadual de Campinas (UNICAMP), Campinas, 2004. 
McGrath, B.P.; Holmes, D.G.; Maiijrekar, M.; Lipo, T.A. An improved modulation strategy for a hybiid multilevel inverter. In: IEEE IAS, p. 2086 - 2093, 2000.

Mei, J.; Shen, K.; Xiao, B.; Tolbert, L. M.; Zheng, J. A New Selective Loop Bias Mapping Phase Disposition PWM with Dynamic Voltage Balance Capability for Modular Multilevel Converter. In: IEEE Transactions on Industrial Electronics, vol. 61, no. 2, feb, 2014.

Meynard, T.; Foch, H. Multi-level conversion: High voltage choppers and voltage source inverters. In: IEEE PESC, p. 397-403, 1992.

Modesto, R. A. Estudo, Projeto e Implementação de Sistemas UPQC/UPS Trifásicos Aplicados no Condicionamento Ativo de Energia Elétrica. In: Escola de Engenharia de São Carlos da Universidade de São Paulo, Tese (Doutorado em Engenharia Elétrica) - Programa de Pós-Graduação em Engenharia Elétrica, 2015.

Modesto, R. A. et al. Power Quality Improvement Using a Dual Unified Power Quality Conditioner/Uninterruptible Power Supply in Three-Phase Four-Wire Systems. In: IET Power Electronics, v. 8, n. 9, p. 1595-1605. ISSN 1755-4535, 2015.

Modesto, R. A.; Silva, S. A. O.; Oliveira, A. A.; Bacon, V. D. A Versatile Unified Power Quality Conditioner Applied to Three-Phase Four-Wire Distribution Systems Using a Dual Control Strategy. IEEE Transactions on Power Electronics, v. 31, no. 8, p. 5503-5514, August, 2016.

Moran, S. A Line Voltage Regulator/Conditioner for Harmonic-Sensitive Load Isolation. In: IEEE Industry Applications Society, p. 947-951, 1989.

Nabae, A.; Takahaski, I.; Akagi, H. A New Neutral-Point-Clamped PWM Inverter. In: IEEE Transactions On Industry Applications, v. 1A-17, n. 5, Set/Oct 1981.

Nasiri, A.; Amac, A. E.; Emadi, A. Series-parallel active filter/uninterruptible power supply system. Electric Power Components and Systems Journal, Chicago, v. 32, n.11, p. 1151-1163, 2004. 
Pan, Z.; Peng, F. Z. Harmonics optimization of the voltage balancing control for multilevel converter/inverter systems. In: IEEE Trans. Power Electron., vol. 21, no. 1, pp. 211-218, Jan. 2006.

Pan, Z.; Peng, F. Z.; Corzine, K. A.; Stefanovic, V. R.; Leuthen, J. M.; Gataric, S. Voltage balancing control of diode-clamped multilevel rectifier/inverter systems. In: IEEE Trans. Ind. Appl., vol. 41, no. 6, pp. 1698-1706, Nov./Dec. 2005.

Paredes, H. K. M. Teoria de Potência Conservativa: Uma Nova Abordagem Para o Controle Cooperativo de Condicionadores de Energia e Considerações Sobre Atribuições de Responsabilidades, Universidade de Campinas, Campinas, Tese de Doutorado, 2011.

Paredes, H. K. M.; Costabeber, A.; Tenti, P. Application of Conservative Power Theory to Cooperative Control of Distributed Compensators in Smart Grids, in International School on Nonsinusoidal Currents and Compensation, Lagów, Polônia, 2010.

Penfield, P; Spencer, R.; Duinker, S. Tellegen's Theorem and Electrical Networks. Cambridge, MA \& London: M.I.T. Press, 1970.

PENG, F. Z. A generalized multilevel inverter topology with self voltage balance. In: IEEE Transactions on Industry Applications, v. 37, n. 2, p. 611-618, Mar.-Apr. 2001.

Pigazo, A. et al. Harmonic Compensation in Shunt Active Power Filters by Applying Kalman Filtering for Estimation of the Averaged Load Conductance. In: IEEE International Symposium On Industrial Electronics. Proceedings... Bari: IEEE, IEEE, 2010. p. 1874-1880, 2010.

Procedimentos de Distribuição de Energia Elétrica no Sistema Elétrico Nacional (2018 - 10ª Revisão). PRODIST, Módulo 8: Qualidade de Energia Elétrica, ANEEL.

Proinfa. Lei ㄲo 10.438 - Programa de Incentivo às Fontes Alternativas de Energia Elétrica, 2002. 
RASHID, M. H. Eletrônica de potência: dispositivos, circuitos e aplicações. Pearson, 4" Edição, 2015.

Rodriguez, J.; Bernet, S.; Steimer, P. K.; Lizama, I. E. A Survey on Neutral-PointClamped Inverters. In: IEEE Transactions on Industrial Electronics, v. 57, n. 7, Jul 2010.

Santos, R. J. M. Condicionador Unificado de Qualidade de Energia Dual com Controle Simplificado. Dissertação (Mestrado) - Universidade do Estado de Santa Catarina, Joinville, SC, 2012.

Santos, R. J. M.; Mezabora, M.; Cunha, J. C. A Dual Unified Power Quality Conditioner Using a Simplified Control Technique. In: XI Congresso Brasileiro de Eletrônica de Potência, 2011.

Santos, R. J. M.; Mezabora, M.; Cunha, J. C. A Simplified Control Technique for a Dual Unified Power Quality Conditioner. In: IEEE Transaction Industrial Electronics, v. 11, n. 61, p. 5851-5860, 2014.

Sayago, J.; Brückner, T.; Bernet, S. How to select the system voltage of MV drives - A comparison of semiconductor expenses. In: IEEE Transaction Industry Electronics, v. 55, n. 9, p. 3381-3390, Sep. 2008.

SERPA, L. A. Current control strategies for multilevel grid connected inverters. Zurich. Doctor of Sciences Thesis, Swiss Federal Institute of Technology, Zurich, 319p, 2007.

Silva, S. A. O. Sistemas de Energia Ininterrupta Line Interactive Trifásicos com Compensação Ativa de Potência Série e Paralela. Tese (Doutorado em Engenharia Elétrica) - Colegiado do Programa de Pós-Graduação em Engenharia Elétrica, Universidade de Minas Gerais, Belo Horizonte, 2001. 
Silva, S. A. O.; Barriviera, R.; Modesto, R. A.; Kaster, M.; Goedtel, A. Single-Phase Power Quality Conditioners with Series-Parallel Filtering Capabilities. In: IEEE 20 $^{\text {th }}$ International Symposium on Industrial Electronics, p. 1124-1130, 2011.

Silva, S. A. O.; Garcia, P. D.; Cortizo, P. C.; Seixas, P. F. A Three-Phase LineInteractive UPS System Implementation with Series-Parallel Active Power-Line Conditioning Capabilities. In: IEEE Transactions on Industry Applications, v.38, n. 6, p. 1581-1590, 2002.

Silva, S. A. O.; Modesto, R. A. Active Power Line Compensation Applied to a ThreePhase Line Interactive UPS System Using SRF Method. In: IEEE/PESC Power Electronics Specialists Conference, p. 2358-2362, 2005.

Steimer, P. K.; Apeldoorn, O.; Odegard, B.; Bernet, S.; Brückner, T. Very high power IGCT PEBB technology. In: IEEE Power Electron. Spec. Conf., pp. 1-7, Recife, Brazil, 2005.

Tallam, R.M.; Naik, R.; Nondahl, T. A. A carrier-based PWM scheme for neutral-point voltage balancing in three-level inverters. In: IEEE Trans. Ind. Appl., vol. 41, no. 6, pp. 1734-1743, Nov./Dec. 2005.

Tedeschi, E. Cooperative Control of Distributed Compensation Systems in Electric Networks Under Non-sinusoidal Operations. Università degli Studi di Padova, Pádua, Itália, Tese de Doutorado, 2009.

Tedeschi, E.; Tenti, P.; Mattavelli, P. Cooperative Design and Control of Distributed Harmonic and Reactive Compensators, in International School on Nonsinusoidal Currents and Compensation, Lagów, Polônia, 2008.

Tedeschi, E.; Tenti, P.; Mattavelli, P. Cooperative Operation of Active Power Filters by Instantaneous Complex Power Control, in Proc. of the $7^{\text {th }}$ Int. Conf. on Power Electronics and Drive Systems (PEDS), Bangkok, 2007. 
Tedeschi, E.; Tenti, P.; Mattavelli, P. Synergistic Control and Cooperative Operation of Distributed Harmonic and Reactive Compensators, in Proc. of the IEEE Power Electronics Conference (PESC), Rhodes, 2008.

Tedeschi, E.; Tenti, P.; Mattavelli, P.; Trombetti, D. Cooperative Control of Electronic Power Processors in Micro-grid, Brazilian Transactions on Power Electronics, vol. 14, ํㅜㄴ, pp. 241-249, 2009.

Teke, A. M.; Meral, M. Cuma, et al. Open Unified Power Quality Conditioner with Control Based on Enhanced Phase Locked Loop. In: IET Generation, Transmission Distribution, v. 3, n. 7, p-254-264, 2013.

Tenti, P.; Mattavelli, P. A Time-Domain Approach to Power Term Definitions under Non-Sinusoidal Conditions, L'Energia Elettrica, vol. 81, pp. 75-84, 2004.

Tenti, P.; Mattavelli, P.; Paredes, H. K. M. Conservative Power Theory, Sequence Components and Accountability in Smart Grids, Przeglad Elektrotechniczny, vol. 6 , pp. 30-37, 2010.

Tenti, P.; Paredes, H. K. M.; Marafão, F. P.; Mattavelli, P. Accountability and Revenue Metering in Smart Micro-Grids, International Workshop of Applied Measurements for Power Systems (AMPS), Achen, Sept 2010.

Tenti, P.; Trombetti, D.; Tedeschi, E.; Mattavelli, P. Compesation of Load Unbalance, Reactive Power and Harmonic Distortion by Cooperative Operation of Distributed Compensators, in European Conference on Power Electronics and Drives, Barcelona, 2009.

Teodorescu, R. et al. Proportional-resonant controllers and filters for grid-connected voltage-source converters. IEE Proceedings Electric Power Applications, v. 153, n. 5, 2006. 
Teymour, H. R.; Sutanto, D.; Muttaqi, K. M.; Ciufo, P. A Novel Modulation Technique and a New Balancing Control Strategy for a Single-Phase Five-Level ANPC Converter. In: IEEE Transactions on Industry Applications, vol. 51, no. 2, mar/apr 2015.

Ucar, M.; Ozdemir, S.; Ozdemir, E. A Four-Leg Unified Series-Parallel Active Filter System for Periodic and Non-Periodic Disturbance Compensation. In: Elsevier Electric Power Systems Research, v. 81, p. 1132-1143, 2011.

Ucar, M; Ozdemir, S. 3-Phase 4-Leg Unified Series-Parallel Active Filter System with Ultracapacitor Energy Storage for Unbalanced Voltage Sag Mitigation. In: Elsevier Electric Power and Energy Systems, v. 49, p. 149-159, 2013.

Vadirajacharya, G.; Kinhal, P.; Agarwal, H.; Gupta, O. Performance Investigation of Neural-Network-Based Unified Power-Quality Conditioner. In: IEEE Transactions on Power Delivery, v. 26, n. 1, 2011.

Vasquez, J. C.; Guerrero, J. M.; Savaghebi, M.; Eloy-Garcia, J.; Teodorescu, R. Modeling, analysis, and design of stationary-reference-frame droop-controlled parallel three-phase voltage source inverters. IEEE Transactions on Industrial Electronics, v. 60 , n. 4, p. 1271-1280, April 2013.

Videt, A.; Moigne, L. P.; Idir, N.; Baudesson, P.; Cimetiere, X. A new carrier-based PWM providing common-mode-current reduction and dc-bus balancing for three-level inverters. In: IEEE Trans. Ind. Electron., vol. 54, no. 6, pp. 3001-3011, Dec. 2007.

Zaragoza, J.; Pou, J.; Ceballos, S.; Robles, E.; Jaen, C.; Corbalan, M. Voltage-balance compensator for a carrier-based modulation in the neutral-point-clamped converter. In: IEEE Trans. Ind. Electron., vol. 56, no. 2, pp. 305-314, Feb. 2009.

Zmood, D.; Holmes, D. Stationary frame current regulation of PWM inverters with zero steady-state error. IEEE Transactions on Power Electronics, v. 18, n. 3, p. 814-822, maio 2003. ISSN 0885-8993. 